\title{
Recreational Mathematics
}

\author{
Paul Yiu \\ Department of Mathematics \\ Florida Atlantic University
}

Summer 2003

Chapters 1-44

Version 031209 


\section{Contents}

1 Lattice polygons 101

1.1 Pick's Theorem: area of lattice polygon . . . . . . . . 102

1.2 Counting primitive triangles . . . . . . . . . . . 103

1.3 The Farey sequence . . . . . . . . . . . . . . . . . 104

2 Lattice points 109

2.1 Counting interior points of a lattice triangle . . . . . 110

2.2 Lattice points on a circle . . . . . . . . . . . . . 111

3 Equilateral triangle in a rectangle 117

3.1 Equilateral triangle inscribed in a rectangle . . . . . . 118

3.2 Construction of equilateral triangle inscribed in a rectangle . . . . . . . . . . . . . . . 119

4 Basic geometric constructions 123

4.1 Geometric mean . . . . . . . . . . . . . . . . . . 124

4.2 Harmonic mean . . . . . . . . . . . . . . . 125

4.3 Equal subdivisions of a segment . . . . . . . . . . . . 126

4.4 The Ford circles . . . . . . . . . . . . . . . 127

5 Greatest common divisor 201

$5.1 \operatorname{gcd}(a, b)$ as an integer combination of $a$ and $b \ldots 202$

5.2 Nonnegative integer combinations of $a$ and $b \ldots 203$

5.3 Cassini formula for Fibonacci numbers . . . . . . . . . 204

5.4 gcd of generalized Fibonacci and Lucas numbers . . . . 205

6 Pythagorean triples 209

6.1 Primitive Pythagorean triples . . . . . . . . . . . . 210

6.2 Primitive Pythagorean triangles with square perimeters 211 
6.3 Lewis Carroll's conjecture on triples of equiareal Pythagorean triangles . . . . . . . . . . . . . . 212

6.4 Points at integer distances from the sides of a primitive Pythagorean triangle . . . . . . . . . . . 213

6.5 Dissecting a rectangle into Pythagorean triangles . . . . 214

7 The tangrams 225

7.1 The Chinese tangram ............... 226

7.2 A British tangram . . . . . . . . . . . . . . 227

7.3 Another British tangram . . . . . . . . . . 228

8 The classical triangle centers $\quad 231$

8.1 The centroid . . . . . . . . . . . . 232

8.2 The circumcircle and the circumcircle ...... . . 233

8.3 The incenter and the incircle . . . . . . . . . 234

8.4 The orthocenter and the Euler line . . . . . . . . 235

8.5 The excenters and the excircles ......... 236

9 The area of a triangle $\quad 301$

9.1 Heron's formula for the area of a triangle . . . . . . . 302

9.2 Heron triangles . . . . . . . . . . . 303

9.3 Heron triangles with consecutive sides . . . . . . . 304

10 The golden section $\quad 309$

10.1 The golden section $\varphi \ldots \ldots$. . . . . . . . . . . . . 310

10.2 Dissection of a square . . . . . . . . . . . 311

10.3 Dissection of a rectangle . . . . . . . . . . 313

10.4 The golden right triangle . . . . . . . . . . . . . 314

10.5 What is the most non-isosceles triangle? . . . . . 316

11 Constructions with the golden section 321

11.1 Construction of golden rectangle . . . . . . . . . . 322

11.2 Hofstetter's compass-only construction of the golden section .................... 323

11.3 Hofstetter's 5-step division of a segment in the golden section . . . . . . . . . . . . 325

11.4 Construction of regular pentagon . . . . . . . . 327

11.5 Ahlburg's parsimonious construction of the regular pentagon . . . . . . . . . . . . . . . 328

11.6 Construction of a regular 17-gon . . . . . . . . 329 
12 Cheney's card trick 335

12.1 Principles ....................... 336

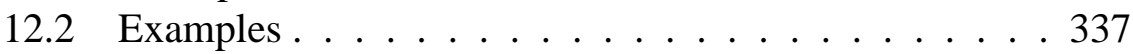

13 Digit problems $\quad 401$

13.1 When can you cancel illegitimately and yet get the correct answer? . . . . . . . . . . . . . 402

13.2 A Multiplication problem . . . . . . . . . . . . . . 403

13.3 A division problem . . . . . . . . . . . . . 404

13.4 The most popular Monthly problem . . . . . . . . . . 407

13.5 The problem of $4 n$ 's . . . . . . . . . . 408

14 Numbers with many repeating digits $\quad \mathbf{4 1 5}$

14.1 A quick multiplication . . . . . . . . . . . . . . . 416

14.2 The repunits . . . . . . . . . . . . . 417

14.3 Squares of repdigits . . . . . . . . . . . 418

14.4 Sorted numbers with sorted squares . . . . . . . . 419

15 Digital sum and digital root $\mathbf{4 2 3}$

15.1 Digital sum sequences . . . . . . . . . . . . . . . . 424

15.2 Digital root . . . . . . . . . . . . . 425

15.3 The digital roots of the powers of $2 \ldots . . . . .426$

15.4 Digital root sequences . . . . . . . . . . . 427

16 3-4-5 triangles in the square 431

17 Combinatorial games $\quad 501$

17.1 Subtraction games . . . . . . . . . . . . . . 502

17.1.1 The Sprague-Grundy sequence . . . . . . . . 503

17.1.2 Subtraction of square numbers . . . . . . . . . 504

17.1.3 Subtraction of square numbers . . . . . . . . 505

17.2 The nim sum of natural numbers . . . . . . . . . 509

17.3 The game Nim . . . . . . . . . . . . . . . . 510

17.4 Northcott's variation of Nim . . . . . . . . . . . 511

17.5 Wythoff's game ............... 512

18 Repunits $\quad \mathbf{5 1 7}$

$18.1 k$-right-transposable integers . . . . . . . . . 518

$18.2 k$-left-transposable integers . . . . . . . . . . . . 519

18.3 Sam Yates' repunit riddles . . . . . . . . . . . 520 
18.4 Recurring decimals . . . . . . . . . . . . 522

18.5 The period length of a prime . . . . . . . . 523

19 More digital trivia

20 The shoemaker's knife $\quad 535$

20.1 Archimedes' twin circles . . . . . . . . . . . 536

20.2 Incircle of the shoemaker's knife . . . . . . . . 537

20.2.1 Archimedes' construction . . . . . . . . . . . 537

20.2.2 Bankoff's constructions . . . . . . . . . 538

20.2.3 Woo's three constructions . . . . . . . . . 539

20.3 More Archimedean circles . . . . . . . . . . . . . . 540

21 Infinitude of prime numbers 601

21.1 Proofs by construction of sequence of relatively prime numbers ................. . . 602

21.2 Somos sequences . . . . . . . . . . . 603

21.3 Fürstenberg's topological proof made easy . . . . . . . 604

22 Strings of prime numbers 611

22.1 The prime number spiral . . . . . . . . . . . 612

22.2 The prime number spiral beginning with $17 \ldots 613$

22.3 The prime number spiral beginning with $41 \ldots 614$

23 Strings of composites

23.1 Strings of consecutive composite numbers . . . . . 622

23.2 Strings of consecutive composite values of $n^{2}+1 \ldots 623$

23.3 Consecutive composite values of $x^{2}+x+41 \ldots 624$

24 Perfect numbers $\quad 627$

24.1 Perfect numbers . . . . . . . . . . . . . 628

24.2 Charles Twigg on the first 10 perfect numbers . . . . . 629

24.3 Abundant and deficient numbers . . . . . . . . . 630

24.3.1 Appendix: Two enumerations of the rational numbers in $(0,1) \ldots \ldots 33$

25 Routh and Ceva theorems 701

25.1 Routh theorem: an example . . . . . . . . . . 702

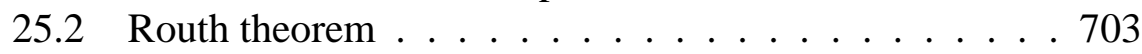

25.3 Ceva Theorem .............. . . 704 
26 The excircles $\quad 711$

26.1 Feuerbach theorem . . . . . . . . . . . 712

26.2 A relation among the radii . . . . . . . . . 713

26.3 The circumcircle of the excentral triangle . . . . . . 714

26.4 The radical circle of the excircles . . . . . . . . 715

26.5 Apollonius circle: the circular hull of the excircles . . . 716

27 Figurate numbers $\quad 719$

27.1 Triangular numbers . . . . . . . . . . . . . . 719

27.2 Special triangular numbers . . . . . . . . . . . 719

27.3 Pentagonal numbers . . . . . . . . . . . . 721

27.4 The polygonal numbers $P_{n, k} \ldots \ldots \ldots 722$

27.4.1 Appendix: Solution of Pell's equation . . . . . 723

28 Polygonal triples $\quad \mathbf{7 2 5}$

28.1 Double ruling of $\mathcal{S} \ldots \ldots \ldots 726$

28.2 Primitive Pythagorean triple associated with a $k$-gonal triple . . . . . . . . . . . . . . 727

28.3 Triples of triangular numbers . . . . . . . . . 727

$28.4 k$-gonal triples determined by a Pythagorean triple . . . 728

28.5 Correspondence between $(2 h+1)$-gonal and $4 h$-gonal triples ................... 730

29 Sums of consecutive squares $\quad \mathbf{8 0 1}$

29.1 Sum of squares of natural numbers . . . . . . . . . . 802

29.2 Sums of consecutive squares: odd number case . . . . . 803

29.3 Sums of consecutive squares: even number case . . . . 805

$\begin{array}{ll}30 \text { Sums of powers of natural numbers } & \mathbf{8 0 7}\end{array}$

31 A high school mathematics contest $\quad 809$

32 Mathematical entertainments $\quad 817$ $32.12 \ldots \ldots \ldots \ldots \ldots . \ldots \ldots 18 \ldots \ldots$

$32.22 \ldots \ldots \ldots 8 \ldots \ldots \ldots$

33 Maxima and minima without calculus 903

34 A British test of teachers' mathematical background 911 
35 A mathematical contest 915

36 Some geometry problems from recent journals 919

37 The Josephus problem and its generalization 1001

37.1 The Josephus problem . . . . . . . . . . . . . . . 1001

37.2 Generalized Josephus problem $\mathcal{J}(n, k) \ldots \ldots 03$

38 Permutations $\quad 1005$

38.1 The universal sequence of permutations . . . . . . . 1005

38.2 The position of a permutation in the universal sequence 1007

39 Cycle decompositions 1009

39.1 The disjoint cycle decomposition of a permutation . . 1009

$39.22 \ldots \ldots \ldots 10 \ldots 10 \ldots \ldots 1 \ldots \ldots$

39.3 Dudeney's Canterbury puzzle 3 . . . . . . . . . . 1013

39.4 The game of ropes and rungs . . . . . . . . . . 1015

40 Graph labelling $\quad 1017$

40.1 Edge-magic triangle . . . . . . . . . . . . . 1017

40.2 Face-magic tetrahedron . . . . . . . . . . . . 1018

40.3 Magic cube . . . . . . . . . . . . . . . 1019

40.4 Edge-magic heptagon . . . . . . . . . . . . . . . . 1020

40.5 Edge-magic pentagram . . . . . . . . . . . . 1021

40.6 A perfect magic circle . . . . . . . . . . . . 1022

41 Card tricks from permutations 1101

42 Tetrahedra 1107

42.1 The isosceles tetrahedron . . . . . . . . . . . 1107

42.2 The volume of an isosceles tetrahedron . . . . . . . . 1108

42.3 Volume of a tetrahedron . . . . . . . . . . . . . 1109

$42.42 \ldots \ldots \ldots \ldots \ldots 1110 \ldots \ldots$

43 Lewis Carroll's unused geometry pillow problem 1113

44 Japanese Temple Geometry 1115 


\section{Chapter 1}

\section{Lattice polygons}

1 Pick's Theorem: area of lattice polygon

2 Counting primitive triangles

3 The Farey sequences

Appendix: Regular solids

Exercise

Project: A cross number puzzle

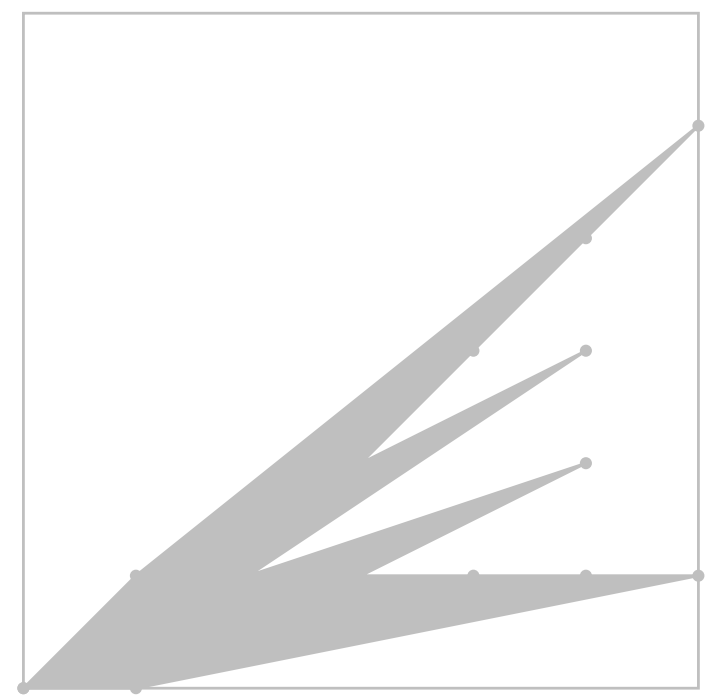




\subsection{Pick's Theorem: area of lattice polygon}

A lattice point is a point with integer coordinates. A lattice polygon is one whose vertices are lattice points (and whose sides are straight line segments). For a lattice polygon, let

$I=$ the number of interior points, and

$B=$ the number of boundary points.

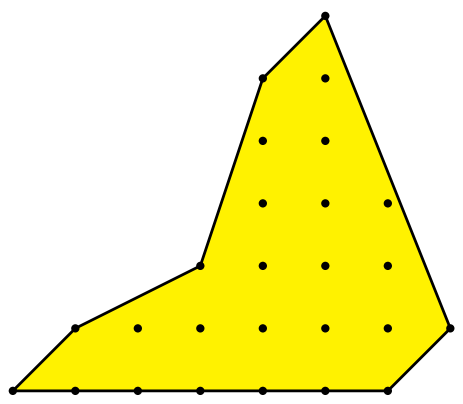

Theorem 1.1 (Pick). The area of a lattice polygon is $I+\frac{B}{2}-1$.

If the polygon is a triangle, there is a simple formula to find its area in terms of the coordinates of its vertices. If the vertices are at the points $\left(x_{1}, y_{1}\right),\left(x_{2}, y_{2}\right),\left(x_{3}, y_{3}\right)$, then the area is ${ }^{1}$

$$
\frac{1}{2}\left|\begin{array}{lll}
x_{1} & y_{1} & 1 \\
x_{2} & y_{2} & 1 \\
x_{3} & y_{3} & 1
\end{array}\right| .
$$

In particular, if one of the vertices is at the origin $(0,0)$, and the other two have coordinates $\left(x_{1}, y_{1}\right),\left(x_{2}, y_{2}\right)$, then the area is $\frac{1}{2}\left|x_{1} y_{2}-x_{2} y_{1}\right|$.

Given a lattice polygon, we can partition it into primitive lattice triangles, i.e., each triangle contains no lattice point apart from its three vertices. Two wonderful things happen that make it easy to find the area of the polygon as given by Pick's theorem.

(1) There are exactly $2 I+B-2$ such primitive lattice triangles no matter how the lattice points are joined. This is an application of Euler's polyhedral formula.

(2) The area of a primitive lattice triangle is always $\frac{1}{2}$. This follows from a study of the Farey sequences.

\footnotetext{
${ }^{1}$ This formula is valid for arbitrary vertices. It is positive if the vertices are traversed counterclockwise, otherwise negative. If it is zero, then the points are collinear.
} 


\subsection{Counting primitive triangles}

We shall make use of the famous Euler polyhedron formula.

Theorem 1.2. If a closed polyhedron has $V$ vertices, $E$ edges and $F$ faces, then $V-E+F=2$.

Given a lattice polygon with a partition into primitive lattice triangles, we take two identical copies and glue them along their common boundary. Imagine the 2-sheet polygon blown into a closed polyhedron. The number of vertices is $V=2 I+B$. Suppose there are $T$ primitive triangles in each sheet. Then there are $F=2 T$ faces of the polyhedron. Since every face is a triangle, and each edge is contained in exactly two faces, we have $2 E=3 F$. It follows that $E=3 T$. Now, Euler's polyhedron formula gives $(2 I+B)-3 T+2 T=2$. From this, we have $T=2 I+B-2$. 


\subsection{The Farey sequence}

Let $n$ be a positive integer. The Farey sequence of order $n$ is the sequence of rational numbers in $[0,1]$ of denominators $\leq n$ arranged in increasing order. We write $0=\frac{0}{1}$ and $1=\frac{1}{1}$.

$$
\begin{aligned}
& \mathcal{F}_{1}: \frac{0}{1}, \frac{1}{1} . \\
& \mathcal{F}_{2}: \frac{0}{1}, \frac{1}{2}, \frac{1}{1} . \\
& \mathcal{F}_{3}: \frac{0}{1}, \frac{1}{3}, \frac{1}{2}, \frac{2}{3}, \frac{1}{1} . \\
& \mathcal{F}_{4}: \frac{0}{1}, \frac{1}{4}, \frac{1}{3}, \frac{1}{2}, \frac{2}{3}, \frac{3}{4}, \frac{1}{1} . \\
& \mathcal{F}_{5}: \frac{0}{1}, \frac{1}{5}, \frac{1}{4}, \frac{1}{3}, \frac{2}{5}, \frac{1}{2}, \frac{3}{5}, \frac{2}{3}, \frac{3}{4}, \frac{4}{5}, \frac{1}{1} . \\
& \mathcal{F}_{6}: \frac{0}{1}, \frac{1}{6}, \frac{1}{5}, \frac{1}{4}, \frac{1}{3}, \frac{2}{5}, \frac{1}{2}, \frac{3}{5}, \frac{2}{3}, \frac{3}{4}, \frac{4}{5}, \frac{5}{6}, \frac{1}{1} . \\
& \mathcal{F}_{7}: \frac{0}{1}, \frac{1}{7}, \frac{1}{6}, \frac{1}{5}, \frac{1}{4}, \frac{2}{7}, \frac{1}{3}, \frac{2}{5}, \frac{3}{7}, \frac{1}{2}, \frac{4}{7}, \frac{3}{5}, \frac{2}{3}, \frac{5}{7}, \frac{3}{4}, \frac{4}{5}, \frac{5}{6}, \frac{6}{7}, \frac{1}{1} .
\end{aligned}
$$

Theorem 1.3. 1. If $\frac{h}{k}$ and $\frac{h^{\prime}}{k^{\prime}}$ are successive terms of $\mathcal{F}_{n}$, then

$$
k h^{\prime}-h k^{\prime}=1 \quad \text { and } \quad k+k^{\prime}>n .
$$

2. If $\frac{h}{k}, \frac{h^{\prime}}{k^{\prime}}$, and $\frac{h^{\prime \prime}}{k^{\prime \prime}}$ are three successive terms of $\mathcal{F}_{n}$, then

$$
\frac{h^{\prime}}{k^{\prime}}=\frac{h+h^{\prime \prime}}{k+k^{\prime \prime}}
$$

The rational numbers in $[0,1]$ can be represented by lattice points in the first quadrant (below the line $y=x$ ). Restricting to the left side of the vertical line $x=n$, we can record the successive terms of the Farey sequence $\mathcal{F}_{n}$ by rotating a ruler about 0 counterclockwise from the positive $x$-axis. The sequence of "visible" lattice points swept through corresponds to $\mathcal{F}_{n}$. If $P$ and $Q$ are two lattice points such that triangle $O P Q$ contains no other lattice points in its interior or boundary, then the rational numbers corresponding to $P$ and $Q$ are successive terms in a Farey sequence (of order $\leq$ their denominators).

Corollary 1.4. A primitive lattice triangle has area $\frac{1}{2}$. 
The Farey polygons $\mathcal{P}_{10}$ and $\mathcal{P}_{20}$
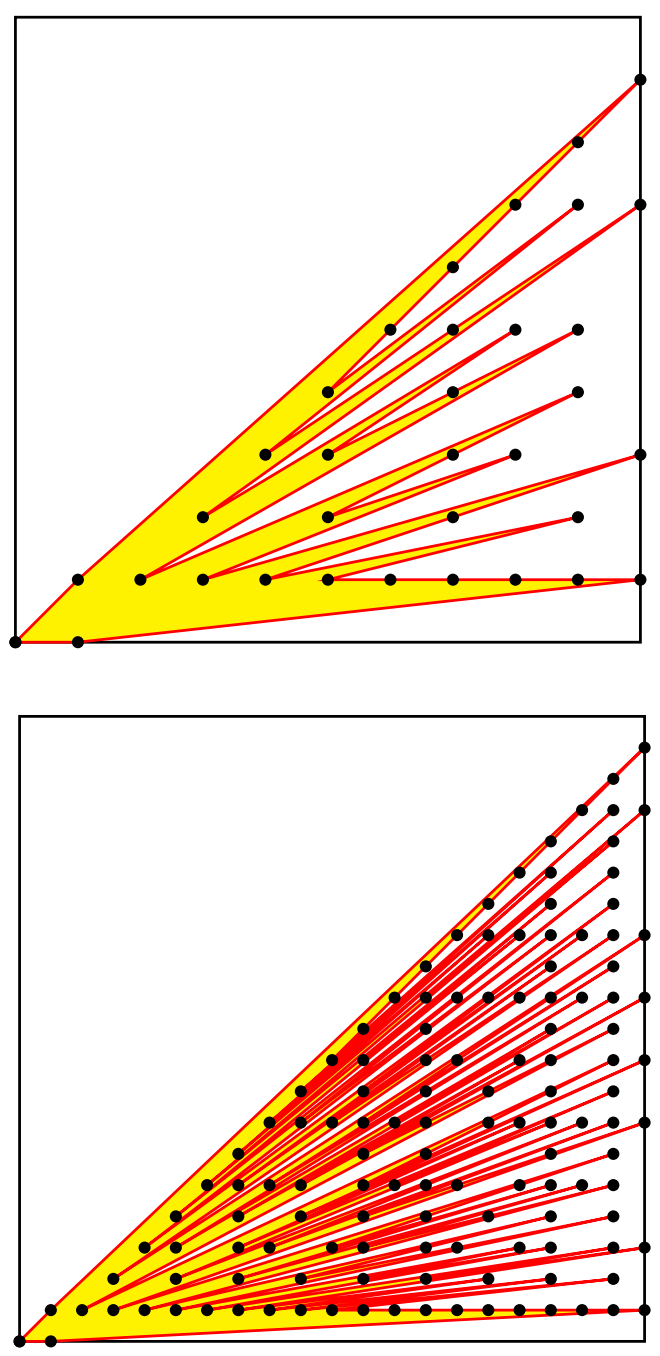

Let $\phi(n)$ be the number of integers $m$ satisfying $1 \leq m \leq n$ and $\operatorname{gcd}(m, n)=1$. The Farey sequence $\mathcal{F}_{n}$ has $1+\sum_{k=1}^{n} \phi(k)$ terms. The polygon $\mathcal{P}_{n}$ contains $2+\sum_{k=1}^{n} \phi(k)$ boundary points and no interior points. By Pick's formula, its area is $A_{n}=\frac{1}{2} \sum_{k=1}^{n} \phi(k)$. By a calculation of D. N. Lehmer [Lehmer], for large values of $n$, this area is about $\frac{3}{2 \pi^{2}}$ of the (smallest) square containing it:

$$
\lim _{n \rightarrow \infty} \frac{A_{n}}{n^{2}}=\frac{3}{2 \pi^{2}}
$$




\section{Appendix: Regular solids}

A regular solid is one whose faces of regular polygons of the same type, say, $n$-gons, and each vertex belongs to the same number of faces, say, $m$ faces. Note that $m \geq 3$ and $n \geq 3$.

Let $V, E$, and $F$ be the numbers of vertices, edges, and faces respectively. Then $n F=2 E=m V$, and $V=\frac{2 E}{m}, F=\frac{2 E}{n}$. Since $V-E+F=2$, we have $\frac{2 E}{m}-E+\frac{2 E}{n}=2$. From this,

$$
E=\frac{2 m n}{2(m+n)-m n}
$$

Since $m, n \geq 3$, and we require $2(m+n)>m n$, the only possibilities are as follows.

\begin{tabular}{|cc|c|c|c|c|}
\hline$m$ & $n$ & $E$ & $V=\frac{2 E}{m}$ & $F=\frac{2 E}{n}$ & regular solid \\
\hline \hline 3 & 3 & & & & tetrahedron \\
\hline 3 & 4 & & & & cube \\
\hline 3 & 5 & & & & duodecahedron \\
\hline 4 & 3 & & & & octahedron \\
\hline 5 & 3 & & & & icosahedron \\
\hline
\end{tabular}




\section{Exercise}

1. Can a lattice triangle be equilateral? Why?

2. Can a lattice polygon be regular? Why? [You may make use of the nontrivial fact that the only values of $n$ for which $\sin \frac{\pi}{n}$ is rational is $n=6$.]

3. For $B=3,4,6,8,9$, give an example of a lattice triangle with exactly one interior point and $B$ boundary points. ${ }^{2}$

4. Give an example of an equilateral lattice hexagon.

5. How many terms does the Farey sequence $\mathcal{F}_{n}$ have? [Hint: Give the answer in terms of the Euler $\varphi$-function].

6. The Farey polygon $\mathcal{P}_{n}$ is the lattice polygon whose vertices, taken in order, are the origin and the points $(k, h)$ for $\frac{h}{k}$ in the Farey sequence $\mathcal{F}_{n}$. Here is $\mathcal{P}_{6}$.

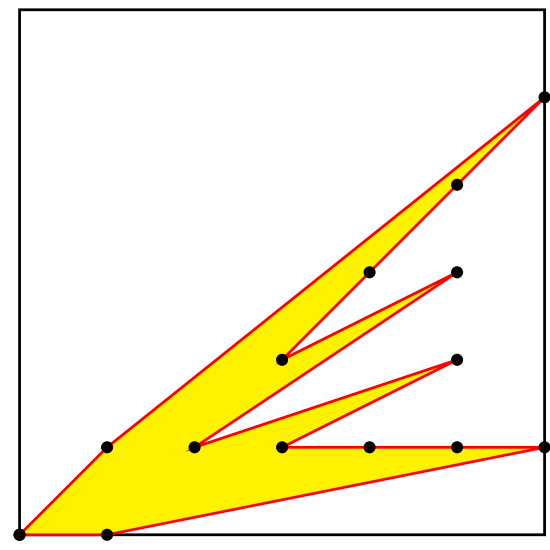

Find the area of $\mathcal{P}_{6}$ and that of $\mathcal{P}_{n}$ for a general $n$.

\footnotetext{
${ }^{2}$ In [Weaver], it is shown that these are the only possible values of $B$ if $I=1$.
} 


\section{A cross number puzzle}

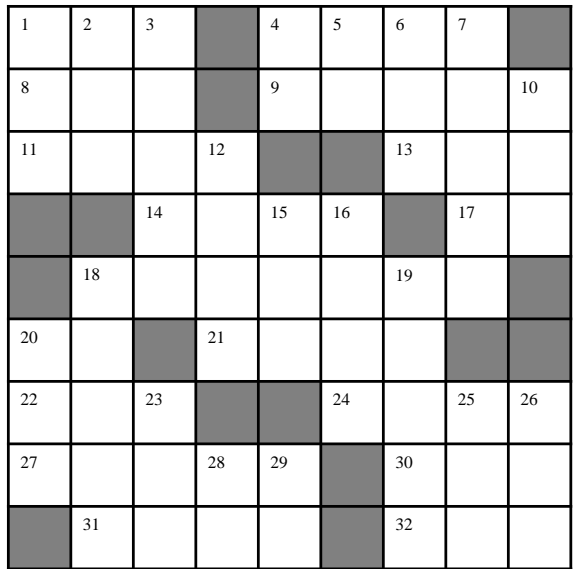

Across

1 A perfect square

4 A palindromic integer

8 This many degrees Fahrenheit is 265 degrees Centigrade

9 With 3-down, a permutation of the digits 0 through 9

11 Second smallest prime of the form $n^{2}+2^{n}+1, n>0$

13 A gross number

14 19-down minus 18-down plus 26-down

17

Number of 2-digit primes

The product of the digits of this number is 78125

20 The sum of this number's positive divisors is 91

$2133^{2}+3^{2}$

22 This number is the sum of the factorials of its digits

24 A power of 6

27 The sum of the fourth power of 5 consecutive triangular numbers

30 A Mersenne prime

31 A power of 2

32 The number of the beast
Down

$1 \quad$ A multiple of 11

2 The product of the positive divisors of the number is $202^{2}$

3 See 9-across

4 A Fermat prime

5 Product of the first 3 primes

6 Colleague of 1-across

7 In base 2, this number is written 11010001110001

10 Yet another perfect square!

12 The first prime year after 1950

15 This many degrees is $\frac{25}{6} \pi$ radians

16 The 17th Fibonacci number

$18210^{2}+111^{2}$

19 The least common multiple of 36 and 1631

20 The number of positive perfect squares less than $10^{5}$

23 The number of positive integers less than 625 which are not divisible by 5

25 The sum of these digits is 15 , and their product is 84

26 Palindromic square

28 The only even prime number

29 20-across minus 28-down 


\section{Chapter 2}

\section{Lattice points}

1 Counting interior points of a lattice triangle

2 Lattice points on a circle

Appendix: The floor and the ceiling

Appendix: Number of lattice points in a circle

Appendix: Number of lattice points under a hyperbola

Exercise

Project: Cross number puzzle

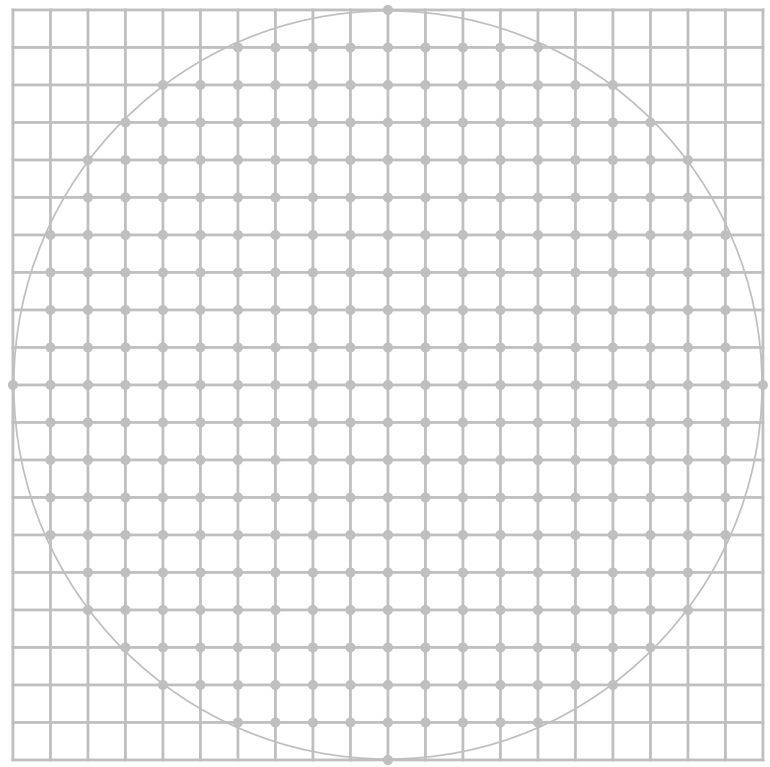




\subsection{Counting interior points of a lattice triangle}

A lattice triangle has vertices at $(0,0),(a, 0)$, and $(a, b)$.

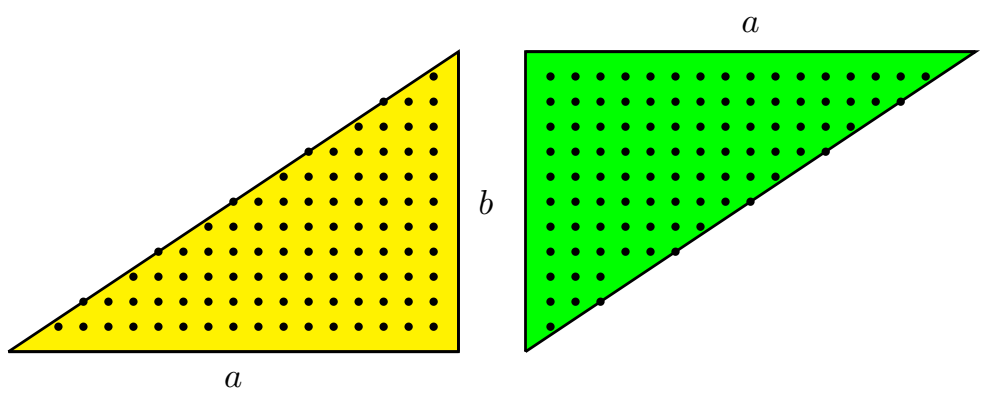

Counting in two ways, we obtain as

$$
\sum_{n=1}^{a-1}\left\lfloor\frac{n b}{a}\right\rfloor=\sum_{m=1}^{b-1}\left\lfloor\frac{m a}{b}\right\rfloor .
$$

Note that each of these is the total number of interior points and those on the hypotenuse (except the two vertices). There are $\operatorname{gcd}(a, b)-1$ points in the latter group.

If we put these two copies together to form a rectangle, we see that the interior points along the diagonal are counted twice. Since the rectangle has $(a-1)(b-1)$ interior points, we have exactly

$$
\frac{(a-1)(b-1)-\operatorname{gcd}(a, b)+1}{2}
$$

lattice points in the interior of the triangle.

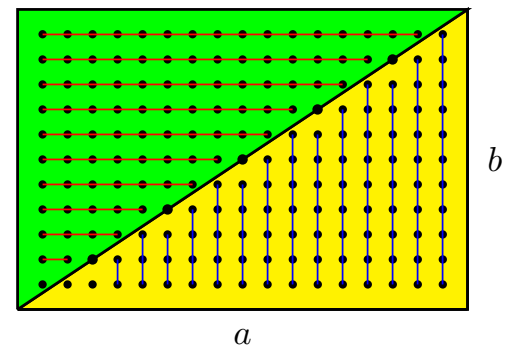

In particular, if $\operatorname{gcd}(a, b)=1$, then the triangle has $\frac{1}{2}(a-1)(b-1)$ interior points.

Note that we have an algorithm for computing the gcd of two integers:

$$
\operatorname{gcd}(a, b)=2 \sum_{m=1}^{b-1}\left\lfloor\frac{m a}{b}\right\rfloor-(a-1)(b-1)+1 \text {. }
$$




\subsection{Lattice points on a circle}

How many lattice points are there on the circle $x^{2}+y^{2}=n^{2}$ ? This number is usually denoted by $r_{2}\left(n^{2}\right)$. The first few values can be read from the following figure.

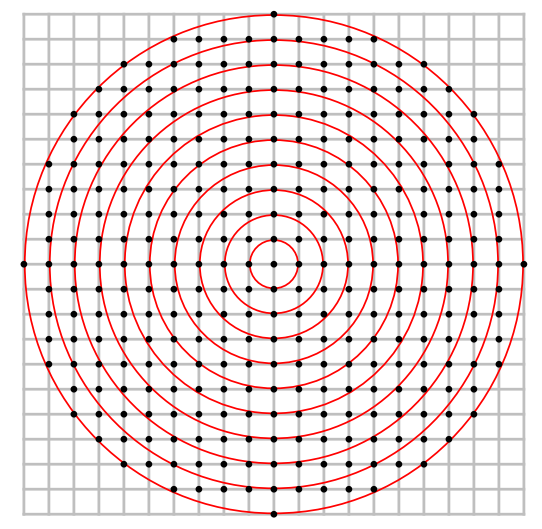

\begin{tabular}{|c||c|c|c|c|c|c|c|c|c|c|}
\hline$n$ & 1 & 2 & 3 & 4 & 5 & 6 & 7 & 8 & 9 & 10 \\
\hline$r_{2}\left(n^{2}\right)$ & & & & & & & & & & \\
\hline
\end{tabular}

In general, if the number $n$ is factored into a product of prime powers

$$
n=2^{a} \cdot \prod_{i} p_{i}^{b_{i}} \cdot \prod_{j} q_{j}^{c_{j}},
$$

where $p_{i}$ and $q_{j}$ are prime numbers of the forms $4 k+1$ and $4 k+3$ respectively, then

$$
r_{2}\left(n^{2}\right)=4 \prod_{i}\left(2 b_{i}+1\right) .
$$

Thus, for example, the circle of radius 100 has lattice points. On the other hand, if $p$ is a prime number of the form $4 n+1$, then the circle of radius $p$ has 12 lattice points. Four of these are on the coordinate axes. The other 8 are of the form $( \pm a, \pm b),( \pm b, \pm a)$, and depend on how the prime $p$ is written as a sum of two squares. 


\section{Appendix: The floor and the ceiling}

The floor of a real number $x$ is the greatest integer not exceeding $x:{ }^{1}$

$$
\lfloor x\rfloor:=\max \{n \in \mathbb{Z}: n \leq x\} .
$$

On the other hand, the ceiling of $x$ is the least integer not exceeded by $x$ :

$$
\lceil x\rceil:=\min \{n \in \mathbb{Z}: n \geq x\} .
$$

If $x$ is not a half-integer, we denote by $\{x\}$ the integer nearest $x$.

\section{Project}

Find all integers $n$ for which $\lfloor\sqrt{n}\rfloor$ divides $n$.

How about $\lceil\sqrt{n}\rceil$ dividing $n$ ?

\footnotetext{
${ }^{1}$ It is sometimes called the greatest integer function of $x$ and denoted by $[x]$.
} 


\section{Appendix: Number of lattice points inside a circle}

Given a real number $r$, how many lattice points are there inside or on the circle of radius $r$, center at the origin?

Write a computer program to find out exactly how many lattice points are inside or on the circle radius 100 .

For large values of $R$, the number $K(R)$ of lattice points inside and on the circle of radius $\sqrt{R}$ satisfies

$$
\pi R-\pi(2 \sqrt{2} \sqrt{R}-2)<K(R)<\pi R+\pi(2 \sqrt{2} \sqrt{R}+2) .
$$

This is often expressed by writing

$$
K(R)=\pi R+O(\sqrt{R}) .
$$

\section{Appendix: Number of lattice points under a hyperbola}

Given a real number $R$, how many lattice points in the first quadrant are under the hyperbola $x y=R$ (but not on the axes)?

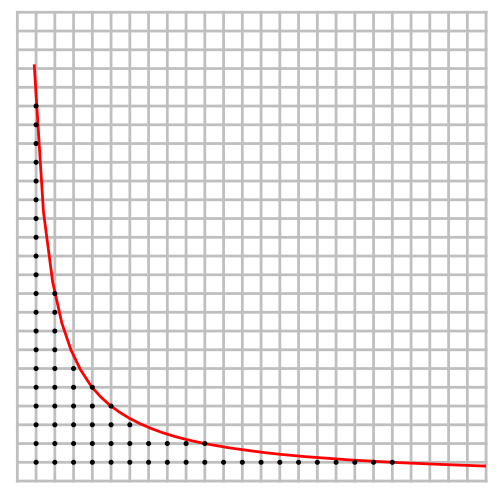

This number is

$$
H(R)=\sum_{1 \leq n \leq\lfloor R\rfloor} d(n) .
$$

As a crude estimate, $H(R)=R \log R+O(R)$. A better estimate was given by Dirichlet

$$
H(R)=R \log R+(2 \gamma-1) R+O(\sqrt{R}) .
$$

Here, $\gamma$ is the Euler constant

$$
\lim _{n \rightarrow \infty}\left(1+\frac{1}{2}+\frac{1}{3}+\cdots+\frac{1}{n}\right)-\log n \approx 0.5772157 \cdots
$$




\section{Exercise}

1. Consider the lattice triangle $A B C$ with $A=(0,0), B=(36,15)$ and $C=(16,30)$. Calculate the lengths of the sides of the triangle. What is the area of the triangle? How many boundary and interior points does it have?

2. Repeat the same for the lattice triangle $A B C$ with $A=(0,0), B=$ $(24,45)$ and $C=(48,55)$.

3. Give an example of a lattice triangle whose side lengths are 13, 14, 15. What is the area? How many interior and boundary points does the triangle have?

4. There is a list of $n$ statements. For $k=1,2, \ldots, n$, the $k$-th statement reads:

The number of false statements in this list is greater than $k$.

Determine the truth value of each of these statements.

5. Solve the equation

$$
\left\lfloor\frac{x+1}{2}\right\rfloor\left\lfloor\frac{x+2}{3}\right\rfloor\left\lfloor\frac{x+3}{4}\right\rfloor=819 .
$$

6. Write 97 as a sum of 2 squares and find the lattice points on the circle of radius 97. 


\section{Project: Cross number puzzle}

Fill in the accompanying square with distinct 2-, 3-, and 4-digit numbers which are perfect squares, none of which begins with 0 .

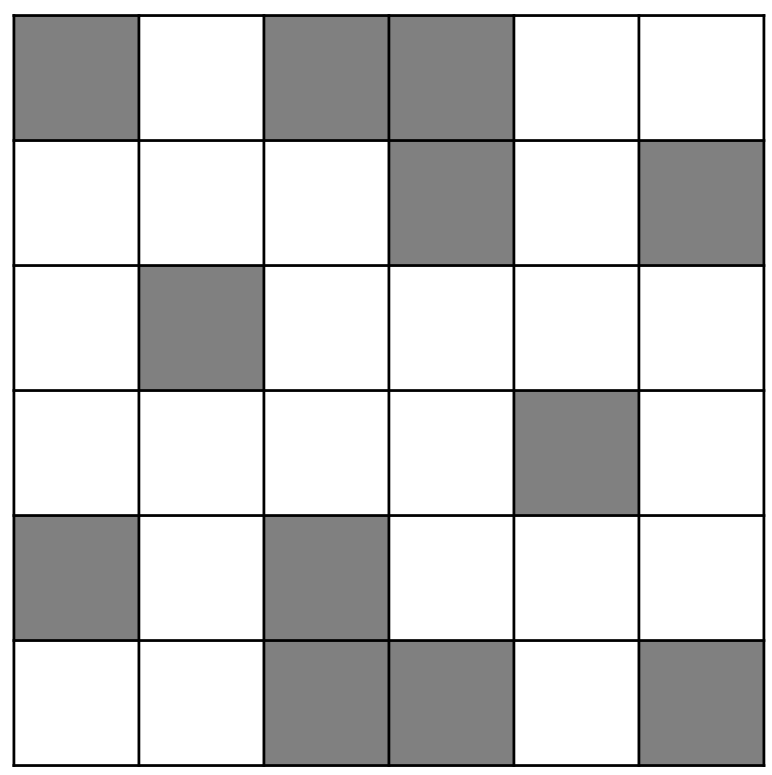




\section{Chapter 3}

\section{Equilateral triangle in a rectangle}

1 Equilateral triangle inscribed in a rectangle

2 Construction

Exercise

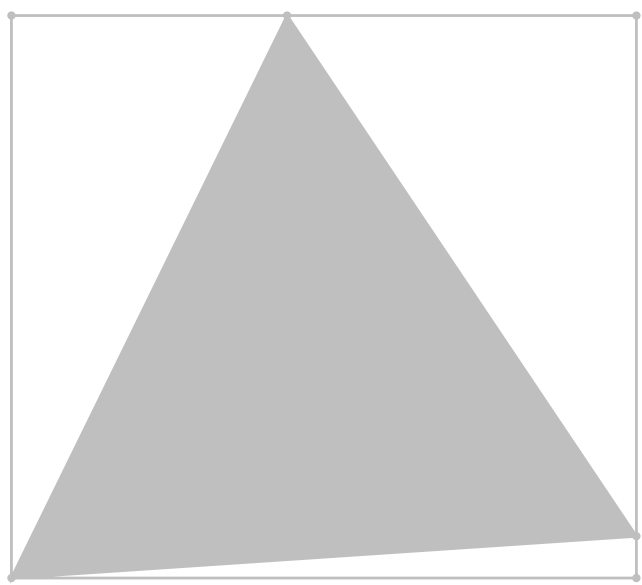




\subsection{Equilateral triangle inscribed in a rectangle}

Given a rectangle $A B C D$, how can we choose a point $P$ on $B C$ and a point $Q$ on $C D$ such that triangle $A P Q$ is equilateral?

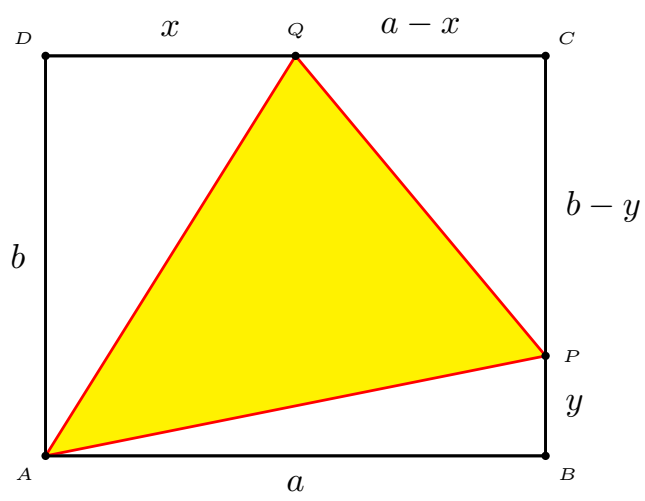

Suppose $A B=D C=a, B C=A D=b, D Q=x$, and $B P=y$. These satisfy

$$
a^{2}+y^{2}=b^{2}+x^{2}=(a-x)^{2}+(b-y)^{2} .
$$

From these, $2(a x+b y)=a^{2}+y^{2}=b^{2}+x^{2}$, and we have

$$
\left(x^{2}-2 a x+b^{2}\right)^{2}=4 b^{2}\left(b^{2}+x^{2}\right)-4 a^{2} b^{2} .
$$

This can be rewritten as

$$
\left(x^{2}+b^{2}\right)\left(\left(x^{2}+b^{2}\right)-4 a x-4 b^{2}\right)=0,
$$

from which

$$
x=2 a-\sqrt{3} b .
$$

Similarly, $y=2 b-\sqrt{3} a$. 


\subsection{Construction of equilateral triangle inscribed in a rectangle}

What is more interesting is that the above calculation leads to a very easy construction of the equilateral triangle $A X Y$.

Construction 3.1. Construct equilateral triangles $B C Y$ and $C D X$ so that $X$ and $Y$ are in the interior of the rectangle. ${ }^{1}$ Join $A X$ to intersect $B C$ at $P$ and $A Y$ to intersect $C D$ at $Q$. Then $A X Y$ is equilateral.

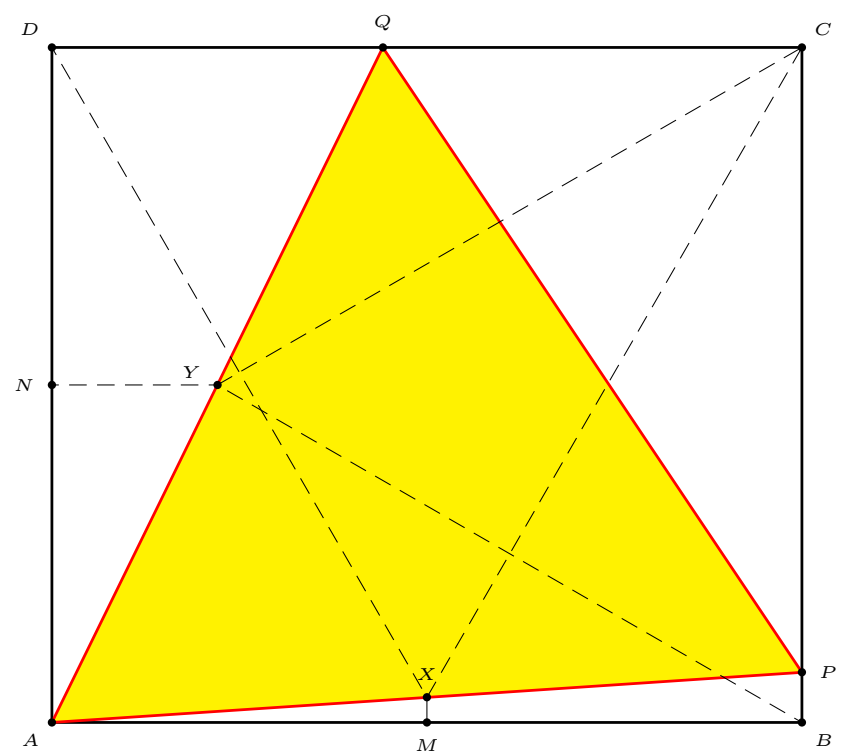

\footnotetext{
${ }^{1}$ This is not always possible. What is the range of the ratio $\frac{a}{b}$ for $X$ and $Y$ to be in the interior of the rectangle?
} 


\section{Exercise}

1. Show that $\operatorname{Area}(A B P)+\operatorname{Area}(A D Q)=\operatorname{Area}(C P Q)$.

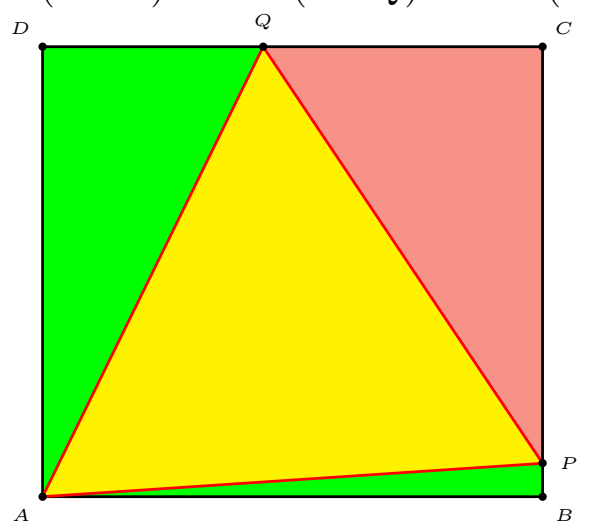

2. Take a $9 \times 10$ rectangle $A B C D$ and obtain the equilateral triangle $A P Q$ by folding.

3. $P$ is a point in the interior of a rectangle $A B C D$. Suppose $A P=a$, $B P=b, C P=c$. Find the distance $D P$ in terms of $a, b, c$.

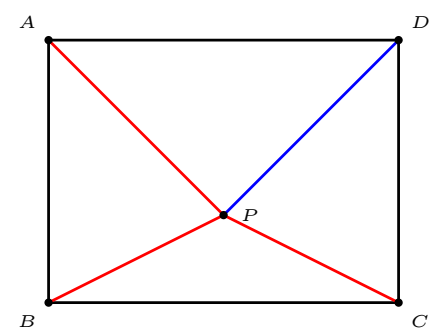

4. A pavement of width $d$ is constructed across a rectangular field of dimensions $a$ by $b$. What is the area of the pavement?

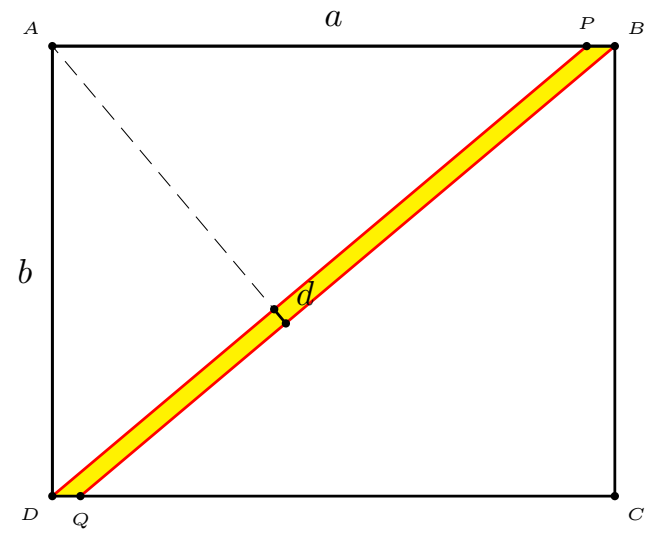


5. A piece of $8 \times 11$ paper is to be cut into a pattern that can be folded into a cube. Find the largest cube that can be obtained, and the percentage of the paper wasted.

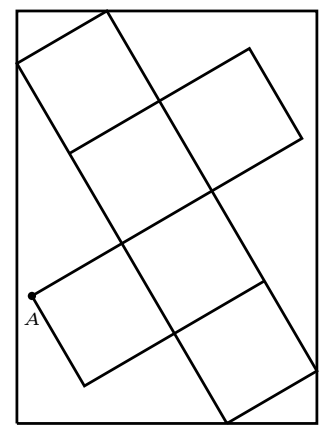

6. What is the length of the paper if the corner $A$ is on the edge, and the width is 8 units?

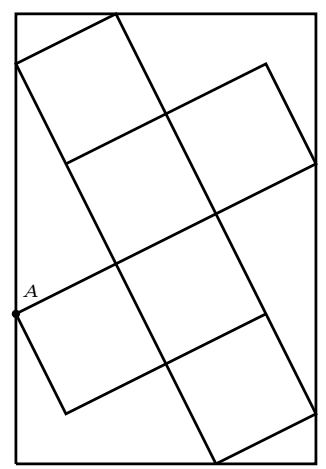




\section{Chapter 4}

\section{Basic geometric constructions}

1 Geometric mean

2 Harmonic mean

3 Equal subdivisions of a segment

4 The Ford circles

Appendix: Some basic construction principles

Appendix: The Geometer's Sketchpad

Exercise

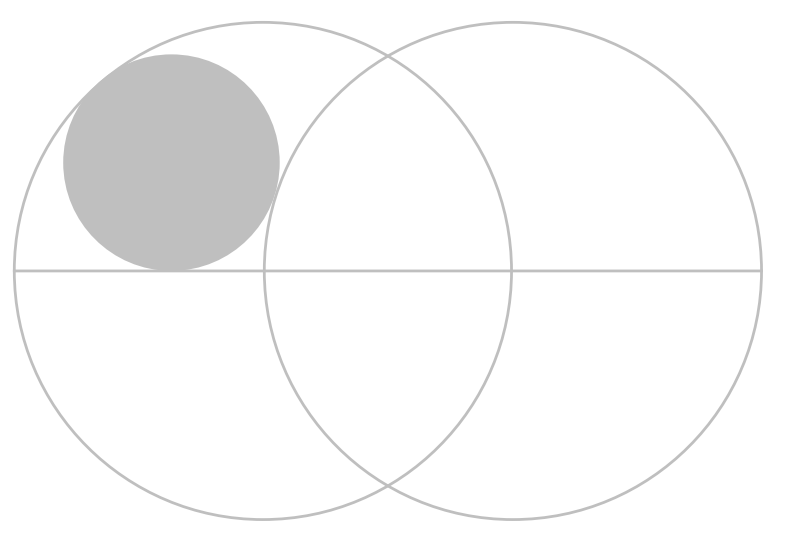




\subsection{Geometric mean}

We present two ruler-and-compass constructions of the geometric means of two quantities given as lengths of segments. These are based on $\mathrm{Eu}-$ clid's proof of the Pythagorean theorem.

Construction 4.1. Given two segments of length $a<b$, mark three points $P, A, B$ on a line such that $P A=a, P B=b$, and $A, B$ are on the same side of $P$. Describe a semicircle with $P B$ as diameter, and let the perpendicular through $A$ intersect the semicircle at $Q$. Then $P Q^{2}=P A \cdot P B$, so that the length of $P Q$ is the geometric mean of $a$ and $b$.

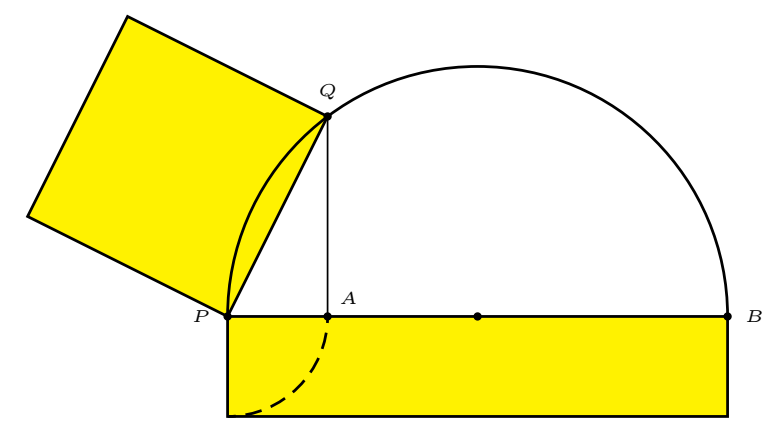

Construction 4.2. Given two segments of length $a, b$, mark three points $A, P, B$ on a line ( $P$ between $A$ and $B$ ) such that $P A=a, P B=b$. Describe a semicircle with $A B$ as diameter, and let the perpendicular through $P$ intersect the semicircle at $Q$. Then $P Q^{2}=P A \cdot P B$, so that the length of $P Q$ is the geometric mean of $a$ and $b$.

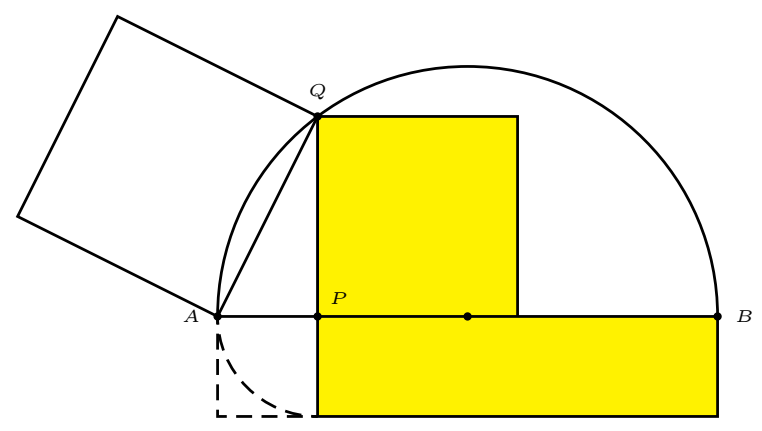




\subsection{Harmonic mean}

Let $A B C D$ be a trapezoid with $A B / / C D$. If the diagonals $A C$ and $B D$ intersect at $K$, and the line through $K$ parallel to $A B$ intersect $A D$ and $B C$ at $P$ and $Q$ respectively, then $P Q$ is the harmonic mean of $A B$ and $C D$ :

$$
\frac{2}{P Q}=\frac{1}{A B}+\frac{1}{C D}
$$

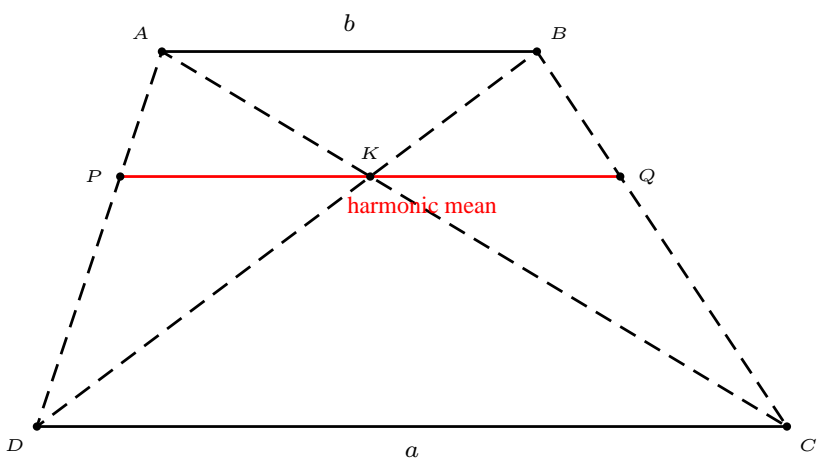

\section{Another construction}

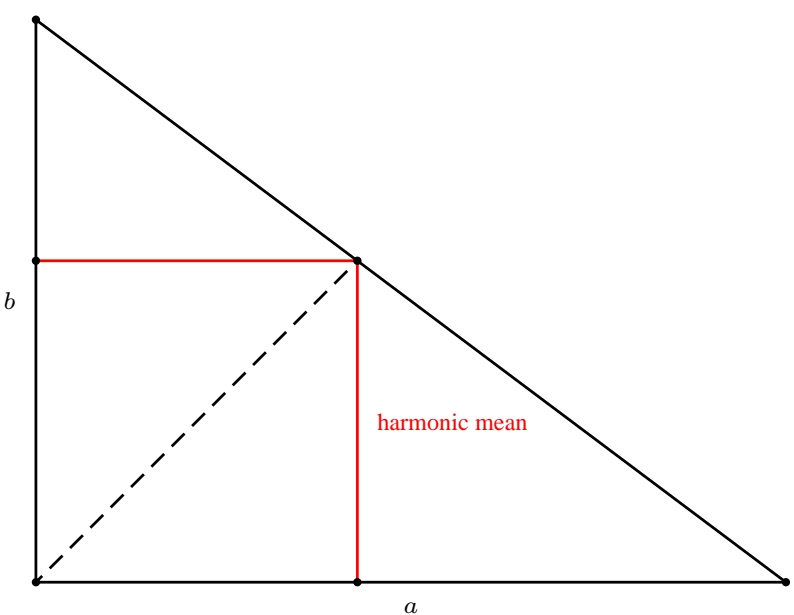




\subsection{Equal subdivisions of a segment}

Here is an algorithm to divide a given segment into $n<2^{k+1}$ equal parts, making use of the binary representation of $n$, which contains not more than $k+1$ digits.

Construct a square $A B C D$ on the given segment. By repeated bisections of $C D$, introduce the points $C_{1}, C_{2}, \ldots, C_{k}$ such that

$$
D C_{i}=\frac{1}{2^{i}} D C, \quad i=1,2, \ldots k .
$$

We also put $C_{0}=C$.

Let $n<2^{k+1}$. Its binary representation has no more than $k+1$ digits. Suppose it has $m+1$ nonzero binary digits.

Along the direction $C D$, relabel those points corresponding to the nonzero digits, as $Q_{0}, Q_{1}, \ldots, Q_{m}{ }^{1}$

Let $P_{0}$ be the (orthogonal) projection of $Q_{0}$ on $A B$.

For each $j=1, \ldots, m$, construct the segments $P_{j-1} D$ and $A Q_{j}$, and mark the projection of their intersection on $A B$ as the point $P_{j}$.

Then $P_{m}$ is the point which divides $A B$ into $n$ equal parts.

Here is the case for $n=13$ with binary representation $1101_{2}$.

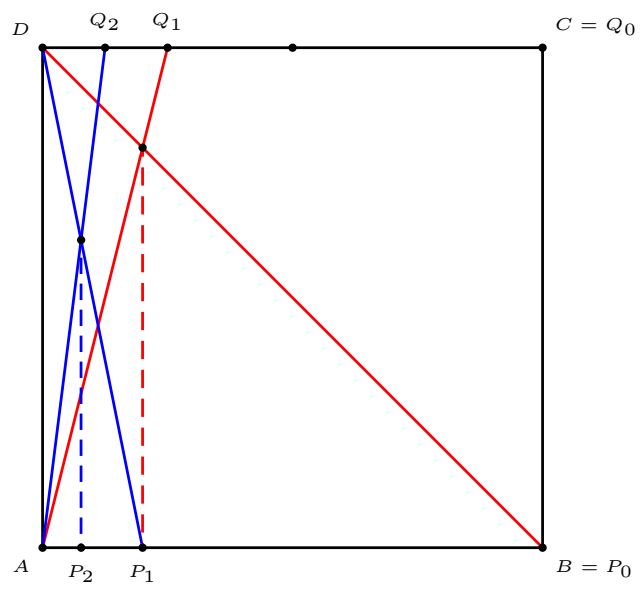

It would be interesting if this procedure can be modified to give a simple construction of subdivision points with ratio $m: n$ instead of $1: n$.

\footnotetext{
${ }^{1}$ For example, if $n=13$, which has binary representation $1101_{2}$, we relabel $C_{0}$ as $Q_{0}, C_{2}$ as $Q_{1}$, and $C_{2}$ as $Q_{2}$.
} 


\subsection{The Ford circles}

The Ford circle of a rational number $r=\frac{p}{q}$ is the circle of radius $\frac{1}{2 q^{2}}$ in the upper half plane tangent to the $x$-axis at the point $(r, 0)$.

The Ford circles of two distinct rational numbers are either disjoint or tangent to each other externally. In the latter case we say that two rational numbers are adjacent.

Theorem 4.1. Two rational numbers are adjacent if and only if they are consecutive terms in a Farey sequence.

Corresponding to each Farey sequence, there is a sequence of tangent Ford circles. Here are the Ford circles corresponding to $\mathcal{F}_{5}$.

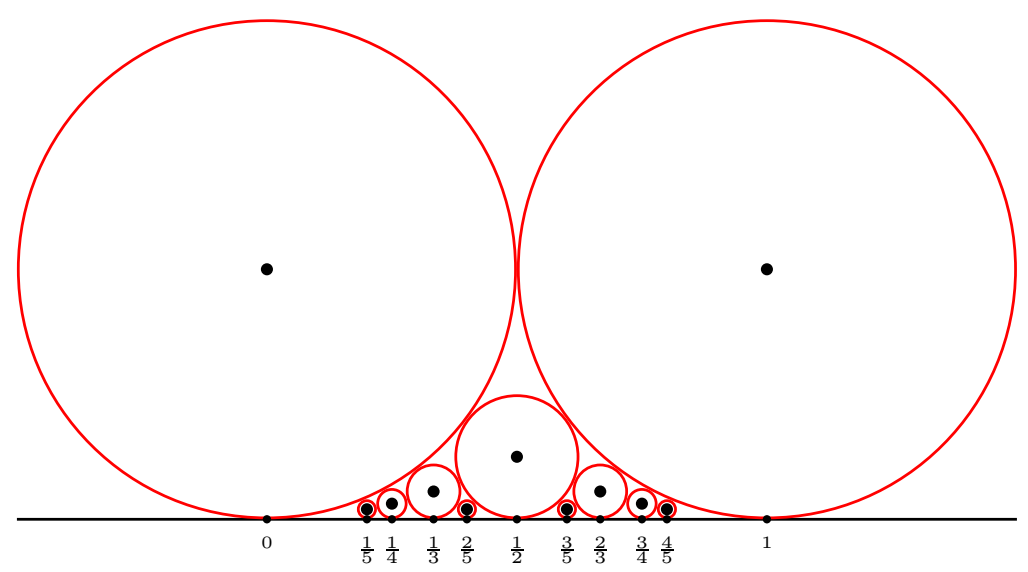

\section{Exercise}

1. If $\frac{p}{q}$ and $\frac{P}{Q}$ are adjacent rational numbers, what is the point of tangency of their Ford circles? 


\section{Appendix: Some basic construction principles}

Theorem 4.2 (Perpendicular bisector locus). Given two distinct $A$ and $B$ on a plane, a point $P$ is equidistant from $A$ and $B$ if and only if $P$ lies on the perpendicular bisector of the segment $A B$.

Theorem 4.3 (Angle bisector locus). A point $P$ is equidistant from two given intersecting lines if and only if it lies on the bisector of an angle between the two lines.

Note that two intersecting lines have two angle bisectors.

Theorem 4.4. If two circles are tangent to each other, the line joining their centers passes through the point of tangency.

The distance between their centers is the sum (respectively difference) of their radii if the tangency is external (respectively internal).
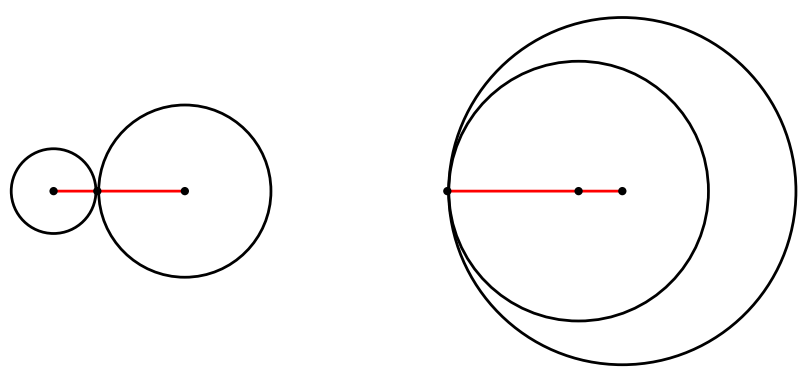


\section{The Geometer's Sketchpad}

Basic commands

- Construct points, segments, lines, circles.

- Construct a segment, ray, or line through two specified points.

- Construct a circle given its center and a point on it. Construct a circle given its center and radius.

- Construct the intersection of two objects.

- Construct the midpoint of a segment.

- Given a line $\ell$ and a point $P$, construct lines perpendicular and parallel to $\ell$ through $P$.

- Translate, rotate, and dilate.

- Hide an object. (If the sketch you are making becomes too complicated, you may wish to hide some of the objects to make it simpler. Note that this is not the same as "delete").

- Label an object. Change the name of the object.

- Save a Sketch.

- Create New Tool.

Toolbox

Open a new sketch. Select a segment and build an equilateral triangle on it. Hide everything except the vertices and sides of the equilateral triangle. Select everything by dragging the mouse from top left to bottom right. Select Create New Tool, and type a name for the tool, say, equilateral triangle. A new tool will now appear.

Save this as a file basic shapes.gsp in the folder tool folder.

From the File menu, choose Document options, Add page, Blank page. On this blank page, construct a square on a segment. Select Create new tool and name this square. Save the file.

You can extend this basic shapes.gsp tool by opening new blank pages and creating new tools. Remember to save the file.

The current basic shape.gsp file contains the following tools: equilateral triangle, square, rectangle, right triangle, parallelogram, rhombus. You may of course add your own tools to the same file. 


\section{Exercise}

1. Given triangle $A B C$, construct the equilateral triangles $B C X, C A Y$ and $A B Z$ externally on the sides of the triangle. Join $A X, B Y$, $C Z$. What can you say about the intersections, lengths, and directions of these lines (segments)?

2. Show that the $90^{\circ}$ angle of a right triangle is bisected by the line joining it to the center of the square on the hypotenuse.

3. Make a sketch to show that for two given positive quantities $a$ and $b$,

$$
\frac{a+b}{2} \geq \sqrt{a b} \geq \frac{2 a b}{a+b} .
$$

4. Construct the following diagram.

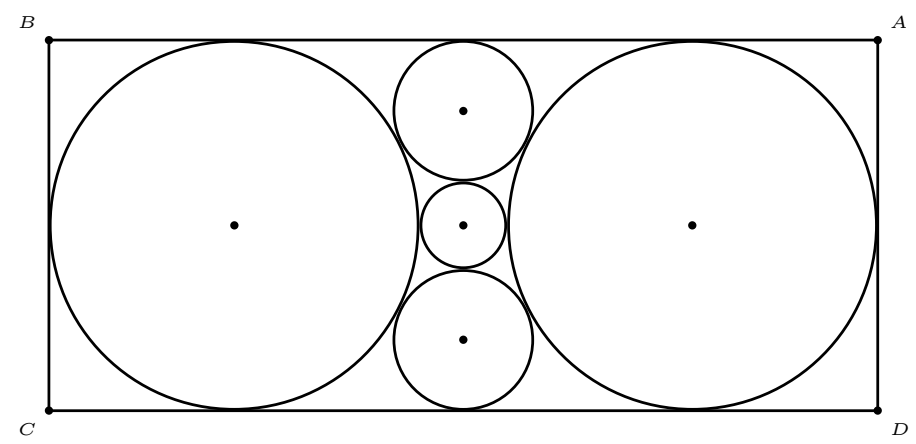

5. Construct the following diagram.

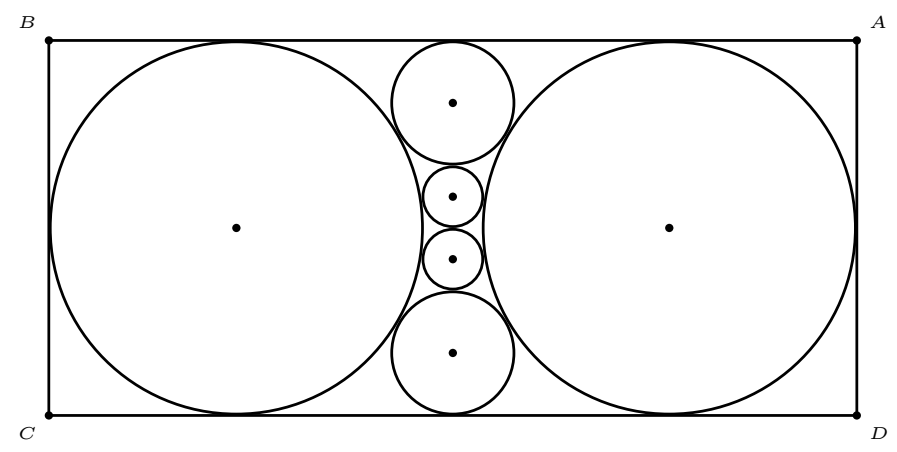


6. Two congruent circles of radii $a$ have their centers on each other. Consider the circle tangent to one of them internally, the other externally, and the line joining their centers. It is known that this circle has radius $\frac{\sqrt{3}}{4} a$. Construct the circle.

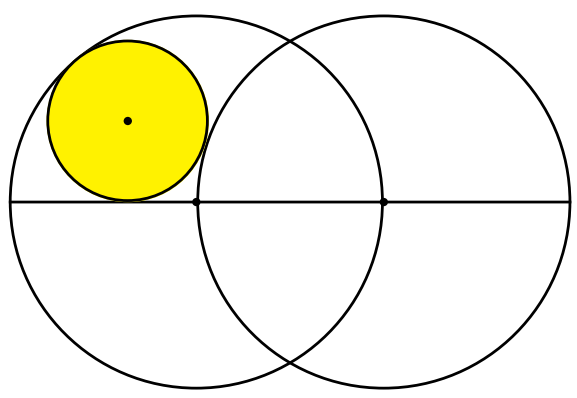

7. An equilateral triangle of side $2 a$ is partitioned symmetrically into a quadrilateral, an isosceles triangle, and two other congruent triangles. If the inradii of the quadrilateral and the isosceles triangle are equal, the common inradius is $(\sqrt{3}-\sqrt{2}) a$. Construct the partition.

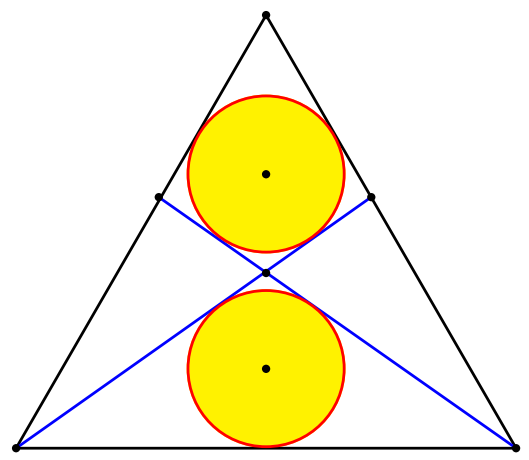

8. Outline a simple procedure to divide a segment into 123 equal parts. 


\section{Chapter 5}

\section{Greatest common divisor}

$1 \operatorname{gcd}(a, b)$ as an integer combination of $a$ and $b$

2 Nonnegative integer combinations of $a$ and $b$

3 Cassini formula for Fibonacci numbers

4 gcd of generalized Fibonacci and Lucas numbers

Appendix: The Euler $\phi$-function

Exercise

Project

\begin{tabular}{|c||c|c|c|c|}
\hline$k$ & $r_{k}$ & $q_{k}$ & $x_{k}$ & $y_{k}$ \\
\hline \hline-1 & $F_{n+1}$ & $* * *$ & 1 & 0 \\
\hline 0 & $F_{n}$ & 1 & 0 & 1 \\
\hline 1 & $F_{n-1}$ & 1 & $F_{1}$ & $-F_{2}$ \\
\hline 2 & $F_{n-2}$ & 1 & $-F_{2}$ & $F_{3}$ \\
\hline 3 & $F_{n-3}$ & 1 & $F_{3}$ & $-F_{4}$ \\
\hline$\vdots$ & & & & \\
\hline$n-3$ & $F_{3}$ & 1 & $(-1)^{n-2} F_{n-3}$ & $(-1)^{n-1} F_{n-2}$ \\
\hline$n-2$ & $F_{2}$ & 1 & $(-1)^{n-1} F_{n-2}$ & $(-1)^{n} F_{n-1}$ \\
\hline$n-1$ & $F_{1}$ & 1 & $(-1)^{n} F_{n-1}$ & $(-1)^{n+1} F_{n}$ \\
\hline$n$ & 0 & $* * *$ & & \\
\hline
\end{tabular}




\section{$5.1 \operatorname{gcd}(a, b)$ as an integer combination of $a$ and $b$}

It is well known that the gcd of two (positive) integers $a$ and $b$ can be calculated efficiently by repeated divisions. Assume $a>b$. We form two sequences $r_{k}$ and $q_{k}$ as follows. Beginning with $r_{-1}=a$ and $r_{0}=b$, for $k \geq 0$, let

$$
q_{k}=\left\lfloor\frac{r_{k-1}}{r_{k}}\right\rfloor, \quad r_{k+1}=\bmod \left(r_{k-1}, r_{k}\right):=r_{k-1}-q_{k} r_{k} .
$$

These divisions eventually terminate when some $r_{n}$ divides $r_{n-1}$. In that case, $\operatorname{gcd}(a, b)=r_{n}$.

If, along with these divisions, we introduce two more sequences $\left(x_{k}\right)$ and $\left(y_{k}\right)$ with the same rule but specific initial values, namely,

$$
\begin{array}{lll}
x_{k+1}=x_{k-1}-q_{k} x_{k}, & x_{-1}=1, & x_{0}=0 ; \\
y_{k+1}=y_{k-1}-q_{k} y_{k}, & y_{-1}=0, & y_{0}=1 .
\end{array}
$$

then we obtain $\operatorname{gcd}(a, b)$ as an integer combination of $a$ and $b:{ }^{1}$

$$
\operatorname{gcd}(a, b)=r_{n}=a x_{n}+b y_{n} .
$$

\begin{tabular}{|c||c|c|c|c|}
\hline$k$ & $r_{k}$ & $q_{k}$ & $x_{k}$ & $y_{k}$ \\
\hline \hline-1 & $a$ & $* * *$ & 1 & 0 \\
\hline 0 & $b$ & $\left\lfloor\frac{a}{b}\right\rfloor$ & 0 & 1 \\
\hline 1 & $a-\left\lfloor\frac{a}{b}\right\rfloor b$ & $\left\lfloor\frac{b}{r_{1}}\right\rfloor$ & $x_{1}$ & $y_{1}$ \\
\hline$\vdots$ & & & & \\
\hline$n-1$ & $r_{n-1}$ & $q_{n-1}$ & $x_{n-1}$ & $y_{n-1}$ \\
\hline$n$ & $r_{n}$ & $q_{n}$ & $x_{n}$ & $y_{n}$ \\
\hline$n+1$ & 0 & & & \\
\hline
\end{tabular}

It can be proved that $\left|x_{n}\right|<b$ and $\left|y_{n}\right|<a$.

Theorem 5.1. Given relatively prime integers $a>b$, there are unique integers $h, k<a$ such that $a k-b h=1$.

Proof. Clearly, $x_{n}$ and $y_{n}$ are opposite in sign. Take $(k, h)=\left(x_{n},-y_{n}\right)$ or $\left(b+x_{n}, a-y_{n}\right)$ according as $x_{n}>0$ or $<0$.

Corollary 5.2. Let $p$ be a prime number. For every integer a not divisible by $p$, there exists a positive integer $b<p$ such that $a b-1$ is divisible by $p$.

\footnotetext{
${ }^{1}$ In each of these steps, $r_{k}=a x_{k}+b y_{k}$.
} 


\subsection{Nonnegative integer combinations of $a$ and $b$}

Find the largest positive integer which cannot as $7 x+11 y$ for integers $x, y \geq 0$.

Let $S:=\{7 x+11 y: x, y$ nonnegative integers $\}$. Arrange the positive integers in the form

$\begin{array}{cccccccccccc}1 & 8 & 15 & 22 * & 29 & 36 & 43 & 50 & 57 & 64 & 71 & \ldots \\ 2 & 9 & 16 & 23 & 30 & 37 & 44 * & 51 & 58 & 65 & 72 & \ldots \\ 3 & 10 & 17 & 24 & 31 & 38 & 45 & 52 & 59 & 66 * & 73 & \ldots \\ 4 & 11 * & 18 & 25 & 32 & 39 & 46 & 53 & 60 & 67 & 74 & \ldots \\ 5 & 12 & 19 & 26 & 33 * & 40 & 47 & 54 & 61 & 68 & 75 & \ldots \\ 6 & 13 & 20 & 27 & 34 & 41 & 48 & 55 * & 62 & 69 & 76 & \ldots \\ 7 & 14 & 15 & 28 & 35 & 42 & 49 & 56 & 63 & 70 & 77 & \ldots\end{array}$

Observations: (i) Every number in the bottom row, being a positive multiple of 7, is in $S$.

(ii) Among the first 11 columns, along each of the first 6 rows, there is a unique entry (with asterisk) which is a multiple of 11 . This entry with asterisk, and those on its right along the row, are in $S$.

(iii) None of the entries on the left of an entry with asterisk is in $S$.

(iv) The entries with asterisks are on different columns.

(v) The rightmost entry with an asterisk is 66. From this, the largest integer not in $S$ is $66-7=59$.

Theorem 5.3. Let $a$ and $b$ be given relatively prime positive integers. Every integer greater than $a b-a-b$ can be written as $a x+b y$ for nonnegative integers $x$ and $y$.

Proof. Let $S:=\{a x+b y: x, y$ nonnegative integers $\}$.

Suppose, for a contradiction, $a b-a-b=a x+b y, x, y \geq 0$. Then $a b=a(x+1)+b(y+1)$. Note that $a \mid b(y+1)$. Since $\operatorname{gcd}(a, b)=1$, we must have $a \mid y+1$. But $y+1$ is a positive integer smaller than $a$. This is clearly a contradiction. From this $a b-a-b \notin S$.

Every integer $t$ in the range $0<t<a$ can be written as $t=a u-b v$ for $0<u<b$ and $0 \leq v<a$. (Choose $u \in\{1,2, \ldots, b-1\}$ such that $a u \equiv t(\bmod b)$. Then $0<a u-t<a b$. It follows that $a u-t=b v$ for some $1 \leq v<a$. Thus, every positive integer $<a+b$ is of the form $a u-b v, 0<u<b, 0 \leq v<a$. Suppose $(a-1)(b-1) \leq n<a b$. Then $a b-n<a+b$. Write $a b-n=a u-b v$ for $0<u<b$ and $0 \leq v<a$. From this, $n=a(b-u)+b v$. This shows that $n \in S$. 


\subsection{Cassini formula for Fibonacci numbers}

The Fibonacci numbers $F_{n}$ are defined recursively by

$$
F_{n+1}=F_{n}+F_{n-1}, \quad F_{0}=0, F_{1}=1 .
$$

The first few Fibonacci numbers are

\begin{tabular}{c|cccccccccccccc}
$n$ & 0 & 1 & 2 & 3 & 4 & 5 & 6 & 7 & 8 & 9 & 10 & 11 & 12 & $\ldots$ \\
\hline$F_{n}$ & 0 & 1 & 1 & 2 & 3 & 5 & 8 & 13 & 21 & 34 & 55 & 89 & 144 & $\ldots$
\end{tabular}

It is easy to see that

$$
\begin{aligned}
\operatorname{gcd}\left(F_{n+1}, F_{n}\right)= & \operatorname{gcd}\left(F_{n}, F_{n-1}\right) \\
= & \operatorname{gcd}\left(F_{n-1}, F_{n-2}\right) \\
& \vdots \\
& =\operatorname{gcd}\left(F_{2}, F_{1}\right)=1 .
\end{aligned}
$$

However, following the euclidean algorithm, we obtain the Cassini formula

$$
F_{n+1} F_{n-1}-F_{n}^{2}=(-1)^{n}
$$

\begin{tabular}{|c||c|c|c|c|}
\hline$k$ & $r_{k}$ & $q_{k}$ & $x_{k}$ & $y_{k}$ \\
\hline \hline-1 & $F_{n+1}$ & $* * *$ & 1 & 0 \\
\hline 0 & $F_{n}$ & 1 & 0 & 1 \\
\hline 1 & $F_{n-1}$ & 1 & $F_{1}$ & $-F_{2}$ \\
\hline 2 & $F_{n-2}$ & 1 & $-F_{2}$ & $F_{3}$ \\
\hline 3 & $F_{n-3}$ & 1 & $F_{3}$ & $-F_{4}$ \\
\hline$\vdots$ & & & & \\
\hline$n-3$ & $F_{3}$ & 1 & $(-1)^{n-2} F_{n-3}$ & $(-1)^{n-1} F_{n-2}$ \\
\hline$n-2$ & $F_{2}$ & 1 & $(-1)^{n-1} F_{n-2}$ & $(-1)^{n} F_{n-1}$ \\
\hline$n-1$ & $F_{1}$ & 1 & $(-1)^{n} F_{n-1}$ & $(-1)^{n+1} F_{n}$ \\
\hline$n$ & 0 & $* * *$ & & \\
\hline
\end{tabular}




\section{4 ged of generalized Fibonacci and Lucas numbers}

Given $a$ and $b$, the generalized Fibonacci sequence $\mathcal{F}(a, b)$

$$
F_{n+1}=a F_{n}+b F_{n-1}, \quad F_{0}=0, F_{1}=1 .
$$

There is also an accompanying Lucas sequence $\mathcal{L}(a, b)$

$$
L_{n+1}=a L_{n}+b F_{n-1}, \quad L_{0}=2, L_{1}=a .
$$

Theorem 5.4. Let $a$ and $b$ be relatively prime integers. The associated Fibonacci and Lucas sequences satisfy

$$
\operatorname{gcd}\left(u_{m}, u_{n}\right)=u_{\operatorname{gcd}(m, n)} .
$$

\section{Examples}

\begin{tabular}{|c|cl|cl|}
\hline$(a, b)$ & $F_{n}^{(a, b)}$ & $L_{n}^{(a, b)}$ & Lucas \\
\hline$(1,1)$ & $F_{n}$ & Fibonacci & $L_{n}$ & Lucas \\
\hline$(1,-1)$ & & periodic & & periodic \\
\hline$(2,-1)$ & $n$ & natural & 2 & constant \\
\hline$(3,-2)$ & $2^{n}-1$ & Mersenne & $2^{n}+1$ & Fermat \\
\hline$(11,-10)$ & $1_{n}$ & repunits & $10_{k-1} 1$ & \\
\hline
\end{tabular}

Corollary 5.5. 1. $\operatorname{gcd}\left(2^{m}-1,2^{n}-1\right)=2^{\operatorname{gcd}(m, n)}-1$.

2. $\operatorname{gcd}\left(F_{m}, F_{n}\right)=F_{\operatorname{gcd}(m, n)}$.

3. $\operatorname{gcd}\left(1_{m}, 1_{n}\right)=1_{\operatorname{gcd}(m, n)}$. 


\section{Exercise}

1. Find the gcd of the following pairs of numbers by completing the second column of each table.

Express the gcd as an integer combination of the given numbers by completing the last two columns.

\begin{tabular}{|c|c|c|c|}
\hline$r_{k}$ & $q_{k}$ & $x_{k}$ & $y_{k}$ \\
\hline \hline 54321 & $* * *$ & 1 & 0 \\
\hline 12345 & & 0 & 1 \\
\hline & & & \\
\hline & & & \\
\hline & & & \\
\hline & & & \\
\hline & & & \\
\hline
\end{tabular}

\begin{tabular}{|c|c|c|c|}
\hline$r_{k}$ & $q_{k}$ & $x_{k}$ & $y_{k}$ \\
\hline \hline 267914296 & $* * *$ & 1 & 0 \\
\hline 196418 & & 0 & 1 \\
\hline & & & \\
\hline & & & \\
\hline & & & \\
\hline & & & \\
\hline & & & \\
\hline
\end{tabular}

2. Find the immediate neighbors of $\frac{13}{31}$ in $\mathcal{F}_{31}$.

3. Find the immediate neighbors of the fraction $\frac{1}{2}$ in the Farey sequence $\mathcal{F}_{n}$.

4. Somebody received a check, calling for a certain amount of money in dollars and cents. When he went to cash the check, the teller made a mistake and paid him the amount which was written as cents, in dollars, and vice versa. Later, after spending $\$ 3.50$, he suddenly realized that he had twice the amount of the money the check called for. What was the amount on the check ? 


\section{Project}

Generalize

$$
\begin{aligned}
& 3+\frac{3}{2}=3 \times \frac{3}{2} . \\
& 3+\frac{3}{2}+\frac{9}{7} \\
& =3 \times \frac{3}{2}+\frac{9}{7} \\
& =3 \times \frac{3}{2} \times \frac{9}{7} \text {. } \\
& 3+\frac{3}{2}+\frac{9}{7}+\frac{81}{67} \\
& =3 \times \frac{3}{2}+\frac{9}{7}+\frac{81}{67} \\
& =3 \times \frac{3}{2} \times \frac{9}{7}+\frac{81}{67} \\
& =3 \times \frac{3}{2} \times \frac{9}{7} \times \frac{81}{67} \\
& 3+\frac{3}{2}+\frac{9}{7}+\frac{81}{67}+\frac{6561}{5623} \\
& =3 \times \frac{3}{2}+\frac{9}{7}+\frac{81}{67}+\frac{6561}{5623} \\
& =3 \times \frac{3}{2} \times \frac{9}{7}+\frac{81}{67}+\frac{6561}{5623} \\
& =3 \times \frac{3}{2} \times \frac{9}{7} \times \frac{81}{67}+\frac{6561}{5623} \\
& =3 \times \frac{3}{2} \times \frac{9}{7} \times \frac{81}{67} \times \frac{6561}{5623}
\end{aligned}
$$




\section{Project}

Write down two 1's, then a 2 between them, then a 3 between any two numbers whose sum is 3 , then a 4 between any two numbers whose sum is 4 , and so forth. How many $n$ 's are there in the sequence? 


\section{Chapter 6}

\section{Pythagorean triples}

1 Primitive Pythagorean triples

2 Primitive Pythagorean triples with square perimeter

3 Lewis Carroll's conjecture on infinity of triples of equiareal Pythagorean triangles

4 Points at integer distances from the sides of a Pythagorean triangle

5 Dissecting a rectangle into Pythagorean triangles

Appendix: Primitive Pythagorean triples $<1000$

Appendix: Dissection of a square

Exercise

Project: Factorable $x^{2}+p x \pm q$

Project: 64 primitive Pythagorean triangles with a common hypotenuse

Project: Primitive Pythagorean triangles with equal perimeters

Project: Two pairs of primitive Pythagorean triples with almost equal perimeters

Project: Cross number puzzle on Pythagorean triples 


\subsection{Primitive Pythagorean triples}

It is well known that every primitive Pythagorean triple $(a, b, c)$ is of the form

$$
a=m^{2}-n^{2}, \quad b=2 m n, \quad c=m^{2}+n^{2}
$$

for relatively prime integers $m$ and $n$ of different parity.

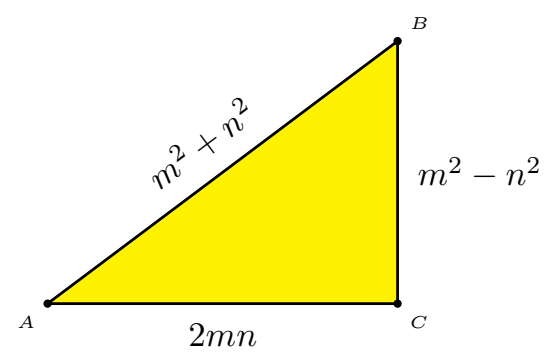

Some basic properties of Pythagorean triples:

1. Exactly one leg is even.

2. Exactly one leg is divisible by 3 .

3. Exactly one side is divisible by 5 .

4. The area is divisible by 6 . Fermat has proved that the area of a Pythagorean triangle can never be a square. Indeed, there is no Pythagorean triangle with two sides whose lengths are square (numbers). 


\subsection{Primitive Pythagorean triangles with square perime- ters}

If $m>n$ are relatively prime integers of opposite parity, they generate a primitive Pythagorean triple $(a, b, c)=\left(m^{2}-n^{2}, 2 m n, m^{2}+n^{2}\right)$ with perimeter $p=2 m(m+n)$. If this perimeter is a square (number), we must have $m=2 q^{2}$ and $m+n=p^{2}$ for some integers $p$ and $q$. From these, $(m, n)=\left(2 q^{2}, p^{2}-2 q^{2}\right)$.

$$
\begin{aligned}
& a=m^{2}-n^{2}=p^{2}\left(4 q^{2}-p^{2}\right), \\
& b=2 m n=4 q^{2}\left(p^{2}-2 q^{2}\right), \\
& c=m^{2}+n^{2}=p^{4}-4 p^{2} q^{2}+8 q^{4} .
\end{aligned}
$$

Note that $p$ is odd, $\lceil\sqrt{2} q\rceil \leq p<2 q$, and $\operatorname{gcd}(p, q)=1$. The perimeter is $4 p^{2} q^{2}=(2 p q)^{2}$.

Here are the first few of such triangles. The last column gives the square root of the perimeter.

\begin{tabular}{|cc|cc|ccc|c|}
\hline$p$ & $q$ & $m$ & $n$ & $a$ & $b$ & $c$ & $2 p q$ \\
\hline 3 & 2 & 8 & 1 & 63 & 16 & 65 & 12 \\
5 & 3 & 18 & 7 & 275 & 252 & 373 & 30 \\
7 & 4 & 32 & 17 & 735 & 1088 & 1313 & 56 \\
9 & 5 & 50 & 31 & 1539 & 3100 & 3461 & 90 \\
11 & 6 & 72 & 49 & 2783 & 7056 & 7585 & 132 \\
11 & 7 & 98 & 23 & 9075 & 4508 & 10133 & 154 \\
13 & 7 & 98 & 71 & 4563 & 13916 & 14645 & 182 \\
13 & 8 & 128 & 41 & 14703 & 10496 & 18065 & 208 \\
15 & 8 & 128 & 97 & 6975 & 24832 & 25793 & 240 \\
13 & 9 & 162 & 7 & 26195 & 2268 & 26293 & 234 \\
17 & 9 & 162 & 127 & 10115 & 41148 & 42373 & 306 \\
17 & 10 & 200 & 89 & 32079 & 35600 & 47921 & 340 \\
19 & 10 & 200 & 161 & 14079 & 64400 & 65921 & 380 \\
$\vdots$ & $\vdots$ & & & & & & \\
\hline
\end{tabular}




\subsection{Lewis Carroll's conjecture on triples of equiareal Pythagorean triangles}

Lewis Carroll conjectured that there is an infinity of Pythagorean triangles with equal areas. If $(p, q, r)$ satisfy $p^{2}+p q+q^{2}=r^{2}$, then the Pythagorean triangles generated by $(r, p),(r, q)$, and $(p+q, r)$ have the same area $\operatorname{pqr}(p+q)$. Since there is an infinity of such triangles, there conjecture is established. This is essentially in Diophantus. 


\subsection{Points at integer distances from the sides of a prim- itive Pythagorean triangle}

Let $(a, b, c)$ be a primitive Pythagorean triangle, with vertices $(a, 0)$, $(0, b)$, and $(0,0)$. The hypotenuse is the line $b x+a y=a b$. The distance of an interior point $(x, y)$ to the hypotenuse is $\frac{1}{c}(a b-b x-a y)$. We seek interior points which are at integer distances from the hypotenuse.

With the parameters $(6,1)$ we have the Pythagorean triangle $(35,12,37)$. Here the five points $(29,1),(23,2),(17,3),(11,4),(5,5)$ are at distances 1 , $2,3,4,5$ from the hypotenuse.

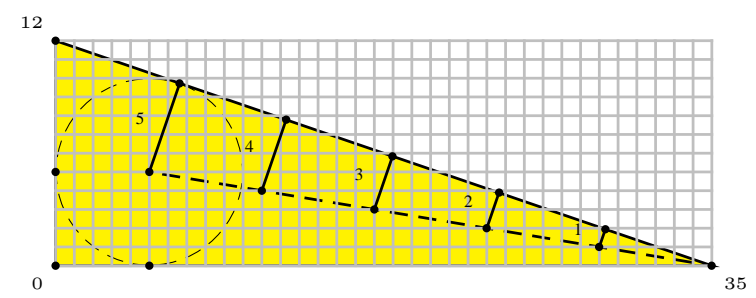

Another example: with paramters $(5,2)$ we have the triangle $(21,20,29)$. Here we have the interior points $(8,11),(16,2),(3,13),(11,4),(6,6),(1,8)$, $(4,1)$ at distances $1,2,3,4,6,8,11$ from the hypotenuse. The arrangement is not as regular as the previous example.

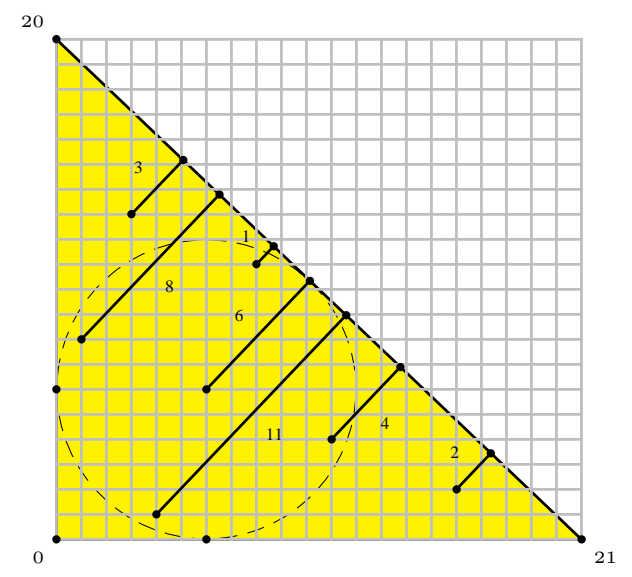




\subsection{Dissecting a rectangle into Pythagorean triangles}

How many matches (of equal lengths) are required to make up the following figure?

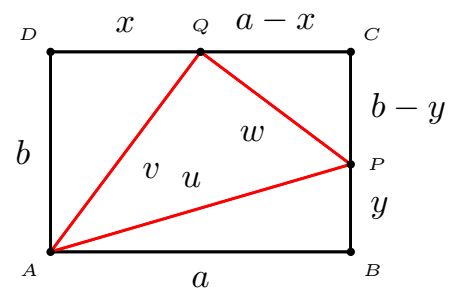

This is Problem 2237 of the Journal of Recreational Mathematics, which asks for the

(i) the smallest such rectangle,

(ii) the smallest such rectangle with $A P=A Q$,

(iii) the smallest such rectangle with $A P Q$ Pythagorean,

(iv) the smallest square.

In (iii), the three right triangles $Q P C, A Q D$ and $A P Q$ are similar. If they are similar to the Pythagorean triangle $a: b: c$, the ratios of similarity are also $a: b: c$. If we put $C P=a^{2}$, then the lengths of the various segments are as shown below. Note that $A B P$ now is a Pythagorean triangle with parameters $b$ and $a$. With $(a, b, c)=(3,4,5)$, we obtain the smallest rectangle satisfying (iv). This also answers (i) since it is clearly also the smallest rectangle (with any restrictions).

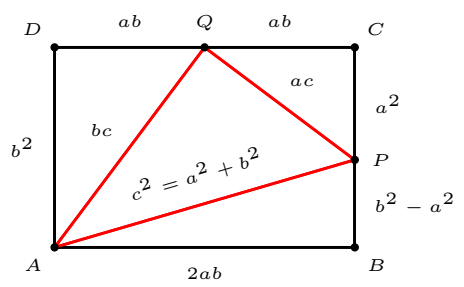

(ii) is also tractable without the help of a computer. Here, we want two Pythagorean triangles with the same hypotenuse. It is well known that the smallest such hypotenuse is 65 . Indeed, $65^{2}=63^{2}+16^{2}=$ $56^{2}+33^{2}$. From this it is easy to complete the data.

The answer to (iv) is given in the Appendix. 
Appendix: Primitive Pythagorean triples $<1000$

\begin{tabular}{|c|c|c|c|c|c|c|c|}
\hline$m, n$ & $a, b, c$ & $m, n$ & $a, b, c$ & $m, n$ & $a, b, c$ & $m, n$ & $a, b, c$ \\
\hline$\overline{22,1}$ & $\overline{33,4,5}$ & $\overline{3,2}$ & 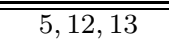 & $\overline{44,1}$ & 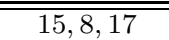 & $\overline{44,3}$ & $\overline{77,24,25}$ \\
\hline 5,2 & $21,20,29$ & 5,4 & $9,40,41$ & 6,1 & $35,12,37$ & 6,5 & $11,60,61$ \\
\hline 7,2 & $45,28,53$ & 7,4 & $33,56,65$ & 7,6 & $13,84,85$ & 8,1 & $63,16,65$ \\
\hline 8,3 & $55,48,73$ & 8,5 & $39,80,89$ & 8,7 & $15,112,113$ & 9,2 & $77,36,85$ \\
\hline 9,4 & $65,72,97$ & 9,8 & $17,144,145$ & 10,1 & $99,20,101$ & 10,3 & $91,60,109$ \\
\hline 10,7 & $51,140,149$ & 10,9 & $19,180,181$ & 11,2 & $117,44,125$ & 11,4 & $105,88,137$ \\
\hline 11,6 & $85,132,157$ & 11,8 & $57,176,185$ & 11,10 & $21,220,221$ & 12,1 & $143,24,145$ \\
\hline 12,5 & $119,120,169$ & 12,7 & $95,168,193$ & 12,11 & $23,264,265$ & 13,2 & $165,52,173$ \\
\hline 13,4 & $153,104,185$ & 13,6 & $133,156,205$ & 13,8 & $105,208,233$ & 13,10 & $69,260,269$ \\
\hline 13,12 & $25,312,313$ & 14,1 & $195,28,197$ & 14,3 & $187,84,205$ & 14,5 & $171,140,221$ \\
\hline 14,9 & $115,252,277$ & 14,11 & $75,308,317$ & 14,13 & $27,364,365$ & 15,2 & $221,60,229$ \\
\hline 15,4 & $209,120,241$ & 15,8 & $161,240,289$ & 15,14 & $29,420,421$ & 16,1 & $255,32,257$ \\
\hline 16,3 & $247,96,265$ & 16,5 & $231,160,281$ & 16,7 & $207,224,305$ & 16,9 & $175,288,337$ \\
\hline 16,11 & $135,352,377$ & 16,13 & $87,416,425$ & 16,15 & $31,480,481$ & 17,2 & $285,68,293$ \\
\hline 17,4 & $273,136,305$ & 17,6 & $253,204,325$ & 17,8 & $225,272,353$ & 17,10 & $189,340,389$ \\
\hline 17,12 & $145,408,433$ & 17,14 & $93,476,485$ & 17,16 & $33,544,545$ & 18,1 & $323,36,325$ \\
\hline 18,5 & $299,180,349$ & 18,7 & $275,252,373$ & 18,11 & $203,396,445$ & 18,13 & $155,468,493$ \\
\hline 18,17 & $35,612,613$ & 19,2 & $357,76,365$ & 19,4 & $345,152,377$ & 19,6 & $325,228,397$ \\
\hline 19,8 & $297,304,425$ & 19,10 & $261,380,461$ & 19,12 & $217,456,505$ & 19,14 & $165,532,557$ \\
\hline 19,16 & $105,608,617$ & 19,18 & $37,684,685$ & 20,1 & $399,40,401$ & 20,3 & $391,120,409$ \\
\hline 20,7 & $351,280,449$ & 20,9 & $319,360,481$ & 20,11 & $279,440,521$ & 20,13 & $231,520,569$ \\
\hline 20,17 & $111,680,689$ & 20,19 & $39,760,761$ & 21,2 & $437,84,445$ & 21,4 & $425,168,457$ \\
\hline 21,8 & $377,336,505$ & 21,10 & $341,420,541$ & 21,16 & $185,672,697$ & 21,20 & $41,840,841$ \\
\hline 22,1 & $483,44,485$ & 22,3 & $475,132,493$ & 22,5 & $459,220,509$ & 22,7 & $435,308,533$ \\
\hline 22,9 & $403,396,565$ & 22,13 & $315,572,653$ & 22,15 & $259,660,709$ & 22,17 & $195,748,773$ \\
\hline 22,19 & $123,836,845$ & 22,21 & $43,924,925$ & 23,2 & $525,92,533$ & 23,4 & $513,184,545$ \\
\hline 23,6 & $493,276,565$ & 23,8 & $465,368,593$ & 23,10 & $429,460,629$ & 23,12 & $385,552,673$ \\
\hline 23,14 & $333,644,725$ & 23,16 & $273,736,785$ & 23,18 & $205,828,853$ & 23,20 & $129,920,929$ \\
\hline 24,1 & $575,48,577$ & 24,5 & $551,240,601$ & 24,7 & $527,336,625$ & 24,11 & $455,528,697$ \\
\hline 24,13 & $407,624,745$ & 24,17 & $287,816,865$ & 24,19 & $215,912,937$ & 25,2 & $621,100,629$ \\
\hline 25,4 & $609,200,641$ & 25,6 & $589,300,661$ & 25,8 & $561,400,689$ & 25,12 & $481,600,769$ \\
\hline 25,14 & $429,700,821$ & 25,16 & $369,800,881$ & 25,18 & $301,900,949$ & 26,1 & $675,52,677$ \\
\hline 26,3 & $667,156,685$ & 26,5 & $651,260,701$ & 26,7 & $627,364,725$ & 26,9 & $595,468,757$ \\
\hline 26,11 & $555,572,797$ & 26,15 & $451,780,901$ & 26,17 & $387,884,965$ & 27,2 & $725,108,733$ \\
\hline 27,4 & $713,216,745$ & 27,8 & $665,432,793$ & 27,10 & $629,540,829$ & 27,14 & $533,756,925$ \\
\hline 27,16 & $473,864,985$ & 28,1 & $783,56,785$ & 28,3 & $775,168,793$ & 28,5 & $759,280,809$ \\
\hline 28,9 & $703,504,865$ & 28,11 & $663,616,905$ & 28,13 & $615,728,953$ & 29,2 & $837,116,845$ \\
\hline 29,4 & $825,232,857$ & 29,6 & $805,348,877$ & 29,8 & $777,464,905$ & 29,10 & $741,580,941$ \\
\hline 29,12 & $697,696,985$ & 30,1 & $899,60,901$ & 30,7 & $851,420,949$ & 31,2 & $957,124,965$ \\
\hline 31,4 & $945,248,977$ & 31,6 & $925,372,997$ & & & & \\
\hline
\end{tabular}




\section{Appendix: Dissection of a square}

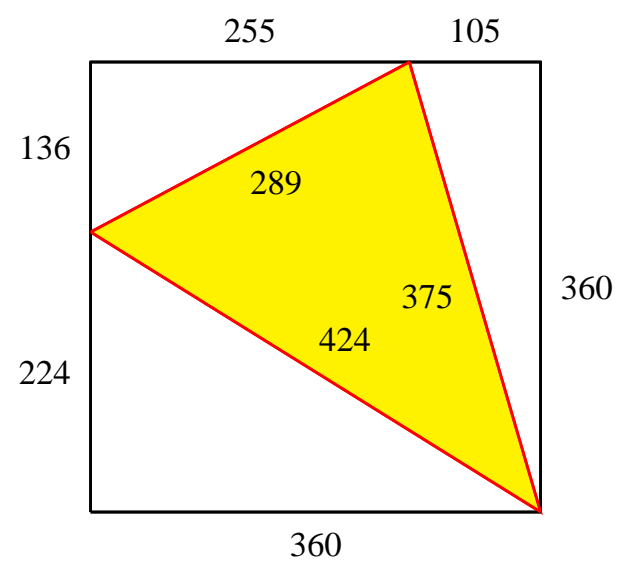




\section{Exercise}

1. A man has a square field, 60 feet by 60 feet, with other property adjoining the highway. He put up a straight fence in the line of 3 trees, at $A, P, Q$. If the distance between $P$ and $Q$ is 91 feet, and that from $P$ to $C$ is an exact number of feet, what is this distance?

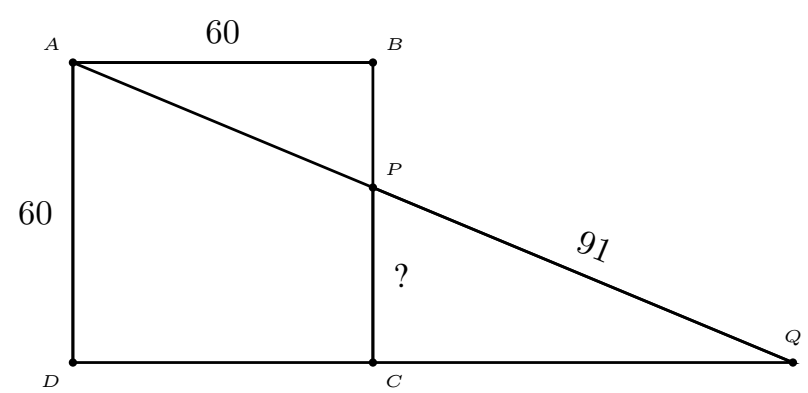

2. What is the least number of matches of equal lengths to make up the following configuration?

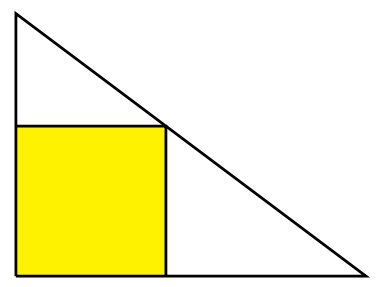

3. What is the least number of matches of equal lengths to make up the following configuration?

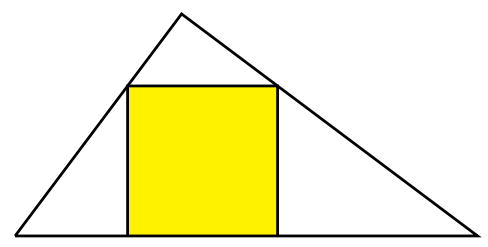

4. Do there exist Pythagorean triangles whose sides are Fibonacci numbers?

5. Give an example of a primitive Pythagorean triangle in which the hypotenuse is a square.

6. Give an example of a primitive Pythagorean triangle in which the even leg is a square. 
7. Give an example of a primitive Pythagorean triangle in which the odd leg is a square. triangle to be a square?

8. Find the shortest perimeter common to two different primitive Pythagorean triangles.

9. Find an integer-sided right angled triangle with sides $x^{2}-1, y^{2}-1$ $z^{2}-1$, where $x, y, z$ are integers.

10. The number of primitive Pythagorean triangle with a fixed inradius is always a power of 2 .

11. Show that there are an infinite number of Pythagorean triangles whose hypotenuse is an integer of the form $3333 \cdots 3$.

12. For each natural number $n$, how many Pythagorean triangles are there such that the area is $n$ times the perimeter ? How many of these are primitive? 
Project: Factorable $x^{2}+p x \pm q$

Theorem 6.1. Let $p$ and $q$ be relatively prime integers. The polynomials $x^{2}+p x \pm q$ are both factorable (over integers) if and only if $|p|$ and $|q|$ are respectively the hypotenuse and area of a primitive Pythagorean triangle. 


\section{Project: 64 primitive Pythagorean triangles with a common hypotenuse}

A product of $n+1$ distint prime numbers of the form $4 k+1$ can be written a sum of two squares in $2^{n}$ different ways, repeatedly by making use of the identity

$$
\left(x_{1}^{2}+y_{1}^{2}\right)\left(x_{2}^{2}+y_{2}^{2}\right)=\left(x_{1} x_{2}-y_{1} y_{2}\right)^{2}+\left(x_{1} y_{2}+x_{2} y_{1}\right)^{2} .
$$

The first 7 primes of the form $4 k+1$ are $5,13,17,29,37,41$, and 53 . Their product is 2576450045 . What are the 64 triples? 
Project: Primitive Pythagorean triangles with equal perimeters

\begin{tabular}{|c|c|c|c|c|c|c|c|}
\hline perimeter & $m+n$ & $2 m$ & $m$ & $n$ & $a$ & $b$ & $c$ \\
\hline \multirow{2}{*}{1716} & 33 & & & & & & \\
\hline & 39 & & & & & & \\
\hline \multirow[t]{3}{*}{14280} & 85 & & & & & & \\
\hline & 105 & & & & & & \\
\hline & 119 & & & & & & \\
\hline \multirow[t]{4}{*}{$\overline{317460}$} & 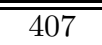 & & & & & & \\
\hline & 429 & & & & & & \\
\hline & 481 & & & & & & \\
\hline & 555 & & & & & & \\
\hline \multirow[t]{5}{*}{1542684} & 899 & & & & & & \\
\hline & 957 & & & & & & \\
\hline & 1023 & & & & & & \\
\hline & 1131 & & & & & & \\
\hline & 1209 & & & & & & \\
\hline \multirow[t]{6}{*}{6240360} & 1785 & & & & & & \\
\hline & 1955 & & & & & & \\
\hline & 1995 & & & & & & \\
\hline & 2185 & & & & & & \\
\hline & 2261 & & & & & & \\
\hline & 2415 & & & & & & \\
\hline \multirow[t]{7}{*}{ "19399380 } & 3135 & & & & & & \\
\hline & 3315 & & & & & & \\
\hline & 3553 & & & & & & \\
\hline & 3705 & & & & & & \\
\hline & 3927 & & & & & & \\
\hline & 4199 & & & & & & \\
\hline & 4389 & & & & & & \\
\hline
\end{tabular}




\section{Project: Two pairs of primitive Pythagorean triples with almost equal perimeters}

In a class in Number Theory the professor gave four students the assignment of finding a fairly large primitive Pythagorean triangle using the well known formula for the legs:

$$
A=2 m n, \quad B=m^{2}-n^{2}, \quad C=m^{2}+n^{2},
$$

where $m$ and $n$ are coprime integers, not both odd. The four students produced four entirely different primitive triangles, but on comparing them it was found that two of them had the same perimeter, while the other two also had the same perimeter, this perimeter differing from the first one by 2 . This interested the class greatly, and much time was spent in an effort to find other such sets, only to discover that there were only four such sets with perimeters less than 500,000. Can you find at least one such set?

\begin{tabular}{|c||c|c|c|c||c|c|c|}
\hline perimeter & $m+n$ & $2 m$ & $m$ & $n$ & $a$ & $b$ & $c$ \\
\hline \multirow{2}{*}{117390} & 273 & & & & & & \\
& 301 & & & & & & \\
\hline \multirow{2}{*}{117392} & 253 & & & & & & \\
\hline & 319 & & & & & & \\
\hline \hline \multirow{2}{*}{313038} & 459 & & & & & & \\
\hline & 527 & & & & & & \\
\hline \multirow{2}{*}{313040} & 455 & & & & & & \\
\hline & 559 & & & & & & \\
\hline \hline \multirow{2}{*}{339150} & 425 & & & & & & \\
& 475 & & & & & & \\
\hline & 525 & & & & & & \\
\hline & 451 & & & & & & \\
\hline & 517 & & & & & & \\
\hline \hline 339152 & 469 & & & & & & \\
\hline & 603 & & & & & & \\
\hline & 437 & & & & & & \\
\hline & 475 & & & & & & \\
\hline & 575 & & & & & & \\
\hline \hline
\end{tabular}




\section{Project: Cross number puzzle on primitive Pythagorean triples ${ }^{1}$}

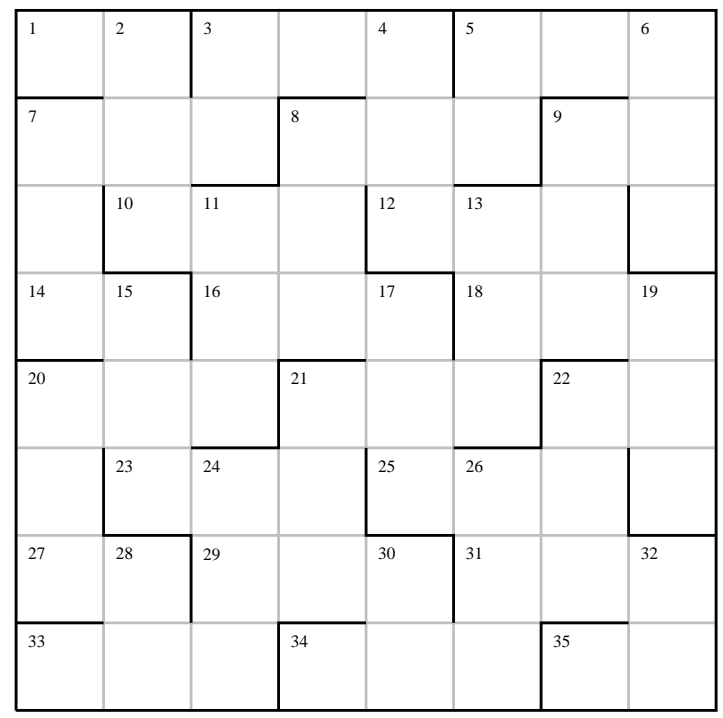

$\begin{array}{ccc}1 B, 3 D, 9 B & 29 B, 7 A, 21 D & 12 B, 11 U, 20 U \\ 2 D, 6 D, 5 B & 19 U, 15 D, 7 D & 22 D, 18 B, 15 U \\ 27 A, 2 D, 26 D & 20 A, 8 D, 8 A & 16 A, 31 A, 33 A \\ 5 D, 3 A, 25 B & 30 D, 14 A, 9 A & 16 B, 24 D, 23 B \\ 28 D, 35 A, 3 U & 30 U, 9 U, 13 D & 22 A, 32 U, 32 D \\ 4 U, 21 A, 21 D & 19 U, 17 D, 10 A & 32 U, 34 A, 33 A\end{array}$

The answers are distinct 2- and 3-digit decimal numbers, none beginning with zero. Each of the above sets of answers is a primitive Pythagorean triple, in increasing size, so that the third member is the hypotenuse.

$A=$ across,$\quad B=$ back, $\quad D=$ down, $\quad U=$ up.

For example, $1 B$ has its tens and units digits in the squares labelled 2 and 1 respectively; $11 U$ is a 3 -digit number with its tens and units digits in squares 16 and 11 respectively.

\footnotetext{
${ }^{1}$ R. K. Guy, Problem 1153, Crux Math., 12 (1986) 139.
} 


\section{Chapter 7}

\section{The tangrams}

1 The Chinese tangram

2 A British tangram

3 Another British tangram

Project

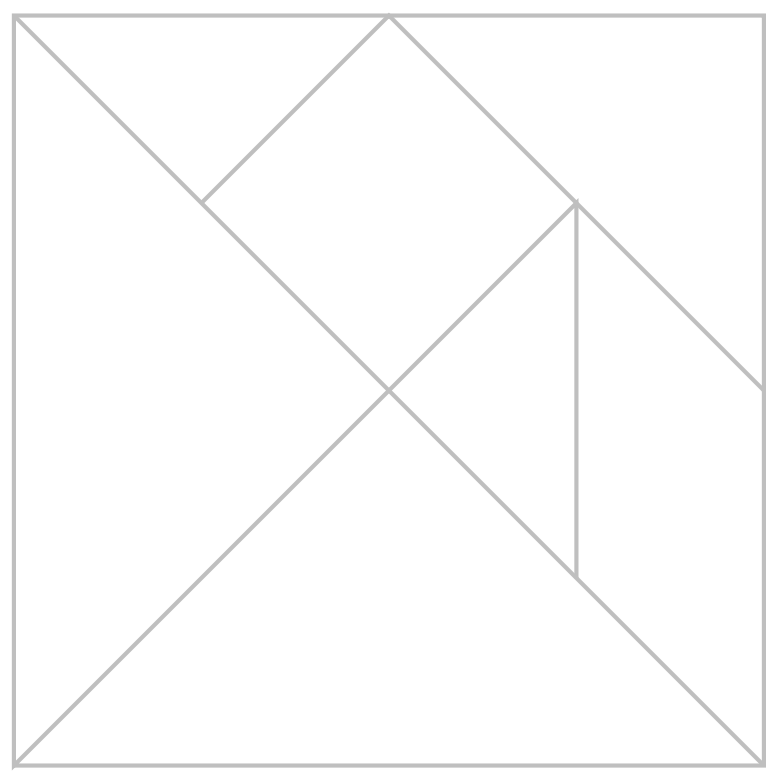




\subsection{The Chinese tangram}

It is known that exactly 13 convex polygons can be formed from the tangram. ${ }^{1}$ Show them. ${ }^{2}$

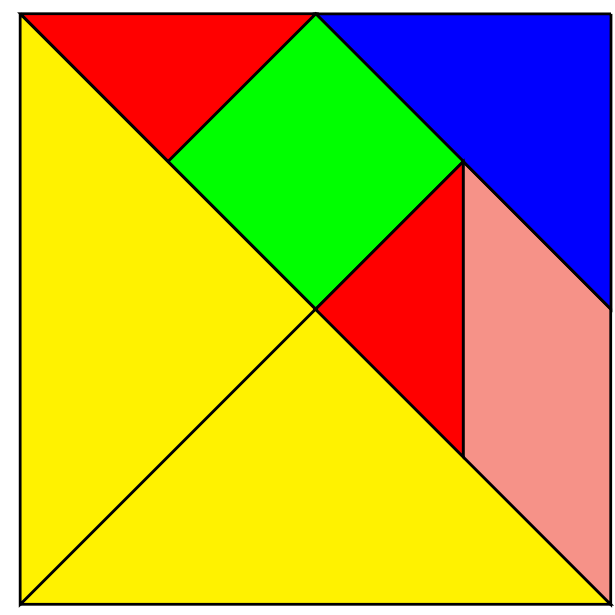

A very interesting book on the Chinese tangram has recently appeared: J. Slocum, The Tangram Book, Sterling, New York, 2003. I finally know what the mysterious Chinese character on p. 45 of Dudeney's Amusements in Mathematics is. The undeciphered Chinese character is zhuó, to drink. The Chinese inscription reads

Liăng rén duì zhuó (Two people having a drink together), č̆ qĩ qiăo zhī huăjǐng yě!! (what a sublimity of the tangram!)

\footnotetext{
${ }^{1}$ F. T. Wang and C. C. Hsiung, A theorem on the tangram, American Math. Monthly, 49 (1942) 596-599.

${ }^{2}$ There are 1 triangle, 6 quadrilaterals, 2 pentagons, and 4 hexagons.
} 


\subsection{A British tangram}

The seven pieces of the puzzle can be fitted together as shown in the figure to make a perfect square. All sloping lines are at $45^{\circ}$, and the lines which intersect the outer square do so at the midpoints of the sides. Find all ways of making a rectangle using all of these pieces. ${ }^{3}$

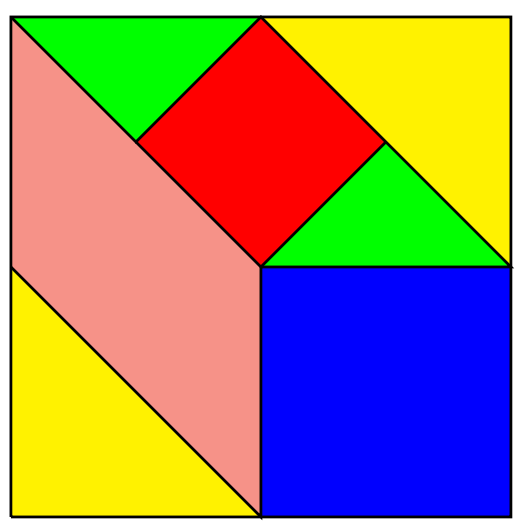

${ }^{3}$ Singmaster, Problem 267, Journal of Recreational Math., 6 (1973) 152-153. 


\subsection{Another British tangram}

Ten pieces of the puzzle can be fitted together as shown in the figure to make $4 \times 5$ rectangle. All sloping lines are at $45^{\circ} .{ }^{4}$

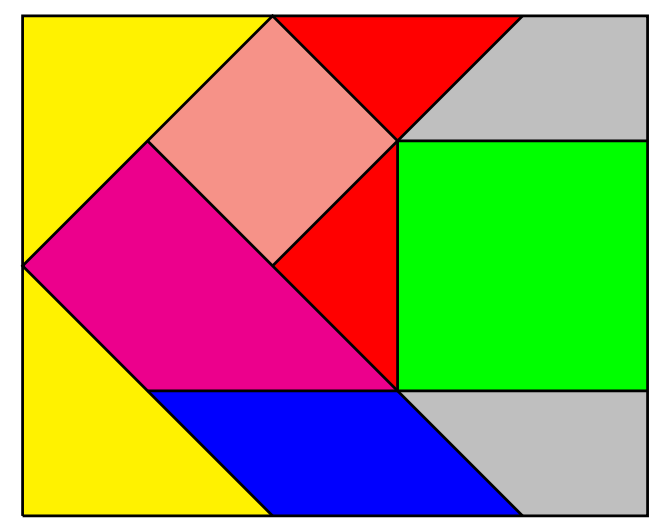

(1) Are there any solution with the green square in a corner or not touching a side?

(2) Prove of disprove: any solution has the two gray trapezoids and the green square in the above relative positions.

(3) Find all solutions.

\footnotetext{
${ }^{4}$ Singmaster, Problem 812, Journal of Recreational Math., 13 (1980-1981) 62-63.
} 


\section{Project}

Show how to dissect a 3-4-5 triangle into 4 pieces that may be rearranged into a square. 5

\footnotetext{
${ }^{5}$ S. Rabinowitz, Problem 1299, Journal Recreational Math., 16 (1983-1984) 139.
} 


\section{Chapter 8}

\section{The classical triangle centers}

1 The centroid

2 The cirumcenter and the circumcircle

3 The incenter and the incircle

4 The orthocenter and the Euler line

5 The excenters and the excircles

Exercise

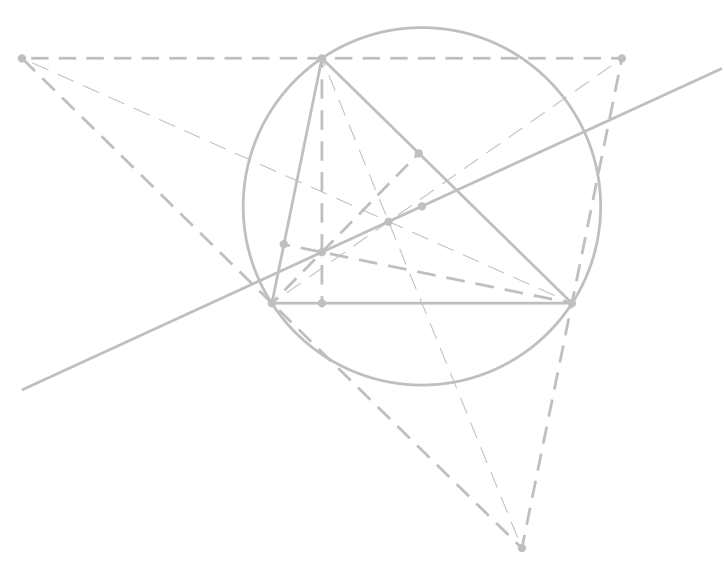


The following triangle centers have been known since ancient times. We shall adopt the following notations. Let $A B C$ be a given triangle. The lengths of the sides $B C, C A, A B$ opposite to $A, B, C$ are denoted by $a, b, c$.

\subsection{The centroid}

The centroid $G$ is the intersection of the three medians. It divides each median in the ratio $2: 1$.

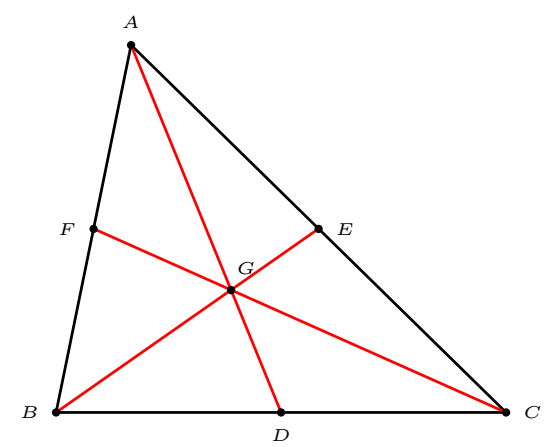

The triangle $D E F$ is called the medial triangle of $A B C$. It is the image of $A B C$ under the homothety $\mathrm{h}\left(G,-\frac{1}{2}\right)$.

The lengths of the medians are given by Apollonius' theorem:

$$
m_{a}^{2}=\frac{1}{4}\left(2 b^{2}+2 c^{2}-a^{2}\right)
$$

etc.

\section{Exercise}

Calculate the lengths of the medians of a triangle whose sidelengths are 136, 170, and 174. 


\subsection{The circumcircle and the circumcircle}

The perpendicular bisectors of the three sides of a triangle are concurrent at the circumcenter of the triangle. This is the center of the circumcircle, the circle passing through the three vertices of the triangle.
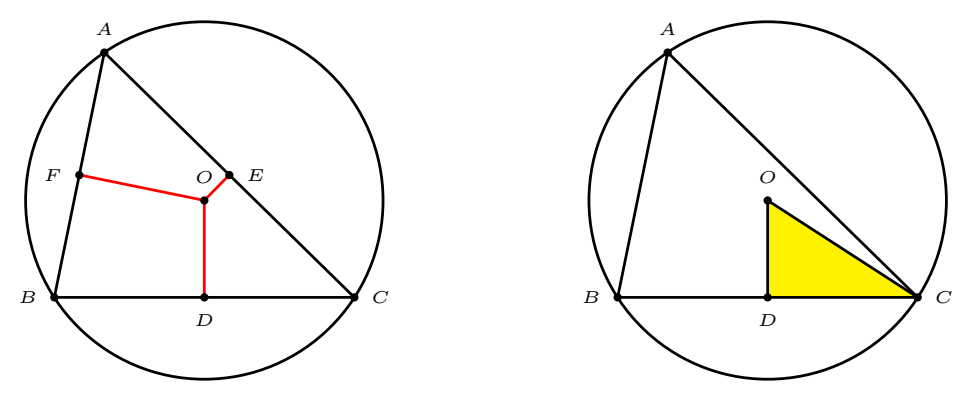

Theorem 8.1 (The law of sines). Let $R$ denote the circumradius of a triangle $A B C$ with sides $a, b$, copposite to the angles $A, B, C$ respectively.

$$
\frac{a}{\sin A}=\frac{b}{\sin B}=\frac{c}{\sin C}=2 R
$$

Since the area of a triangle is given by $\triangle=\frac{1}{2} b c \sin A$, the circumradius can be written as

$$
R=\frac{a b c}{4 \triangle}
$$




\subsection{The incenter and the incircle}

The internal angle bisectors of a triangle are concurrent at the incenter of the triangle. This is the center of the incircle, the circle tangent to the three sides of the triangle.

If the incircle touches the sides $B C, C A$ and $A B$ respectively at $X$, $Y$, and $Z$,

$$
A Y=A Z=s-a, \quad B X=B Z=s-b, \quad C X=C Y=s-c .
$$

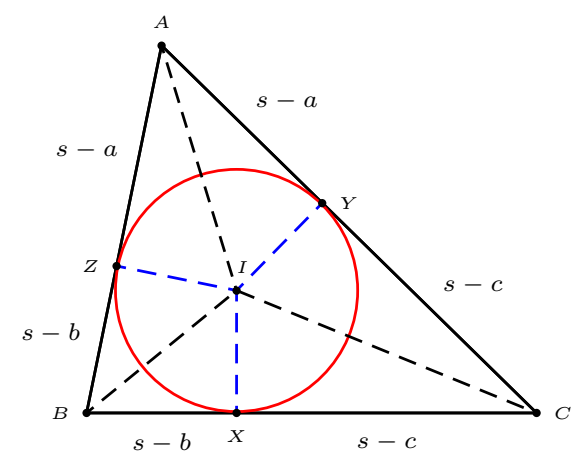

Denote by $r$ the inradius of the triangle $A B C$.

$$
r=\frac{2 \triangle}{a+b+c}=\frac{\triangle}{s} .
$$




\subsection{The orthocenter and the Euler line}

The orthocenter $H$ is the intersection of the three altitudes of triangle $A B C$. These altitudes can be seen as the perpendicular bisectors of the antimedial triangle $X Y Z$ of $A B C$, which is bounded by the three lines each passing through $A, B, C$ parallel to their respective opposite sides.

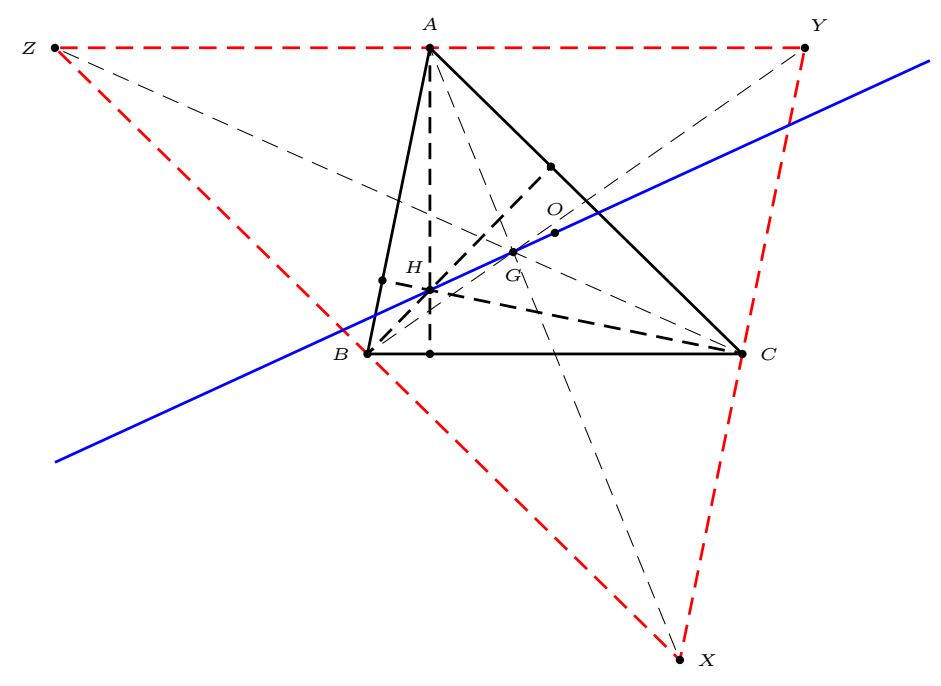

$X Y Z$ is the image of triangle $A B C$ under the homothety $\mathrm{h}(G,-2)$. It follows that $H$ is the image of $O$ under the same homothety. We conclude that $O, G$, and $H$ are collinear, and $O G: G H=1: 2$.

The line containing $O, G, H$ is the famous Euler line of triangle $A B C$. 


\subsection{The excenters and the excircles}

The internal bisector of each angle and the external bisectors of the remaining two angles are concurrent at an excenter of the triangle. An excircle can be constructed with this as center, tangent to the lines containing the three sides of the triangle.

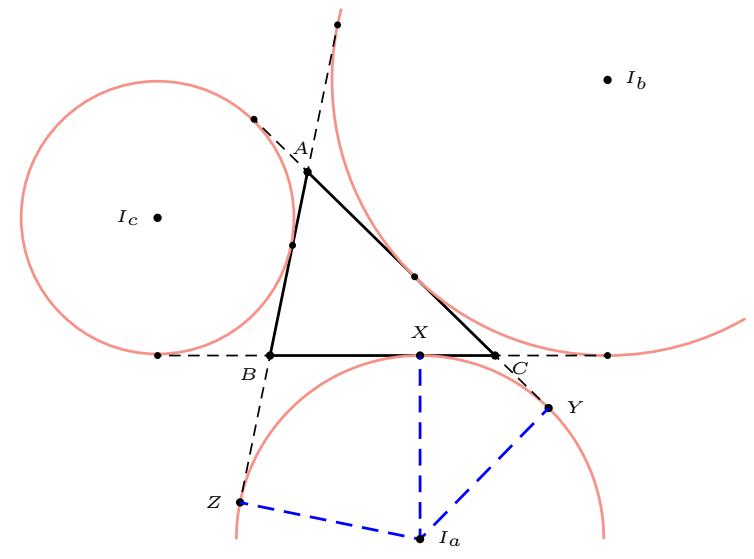

The exradii of a triangle with sides $a, b, c$ are given by

$$
r_{a}=\frac{\triangle}{s-a}, \quad r_{b}=\frac{\triangle}{s-b}, \quad r_{c}=\frac{\triangle}{s-c} .
$$




\section{Exercise}

1. Given a triangle $A B C$, construct a triangle whose sides have the same lengths as the medians of $A B C$.

2. Construct the incircle of triangle $A B C$, and mark the points of contact $X$ on $B C, Y$ on $C A$, and $Z$ on $A B$. Are the lines $A X, B Y$, $C Z$ concurrent? If so, is their intersection the incenter of triangle $A B C$ ?

3. Given a rectangle $A B C D$, construct points $P$ on $B C$ and $Q$ on $C D$ such that the triangle $A P Q$ is equilateral.

4. Let $D, E, F$ be the midpoints of $B C, C A, A B$ of triangle $A B C$. Construct the circumcircle of $D E F$. This is called the nine-point circle of triangle $A B C$. Construct also the incircle of triangle $A B C$. What do you observe about the two circles? How would you justify your observation?

5. Construct the circle through the excenters of triangle $A B C$. How is its center related to the circumcenter and incenter of triangle $A B C$ ?

6. Given three non-collinear points as centers, construct three circles mutually tangent to each other externally. 


\section{Chapter 9}

\section{The area of a triangle}

1 Heron's formula for the area of a triangle

2 Heron triangles

3 Heron triangles with consecutive sides

Appendix: Heron triangles with sides $<1000$

Appendix: A famous unsolved problem

Project: Triangles whose sides and one altitude are in arithmetic progression

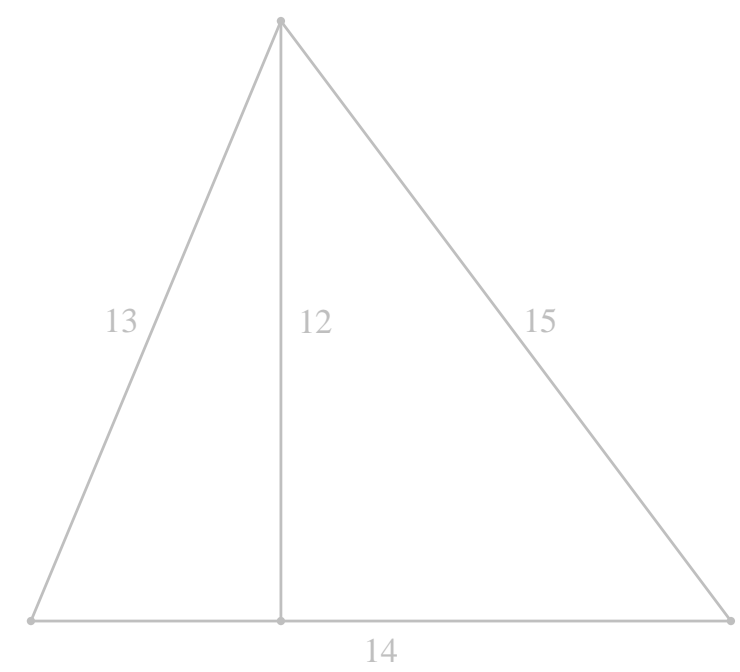




\subsection{Heron's formula for the area of a triangle}

Theorem 9.1. The area of a triangle of sidelengths $a, b, c$ is given by

$$
\triangle=\sqrt{s(s-a)(s-b)(s-c)},
$$

where $s=\frac{1}{2}(a+b+c)$.

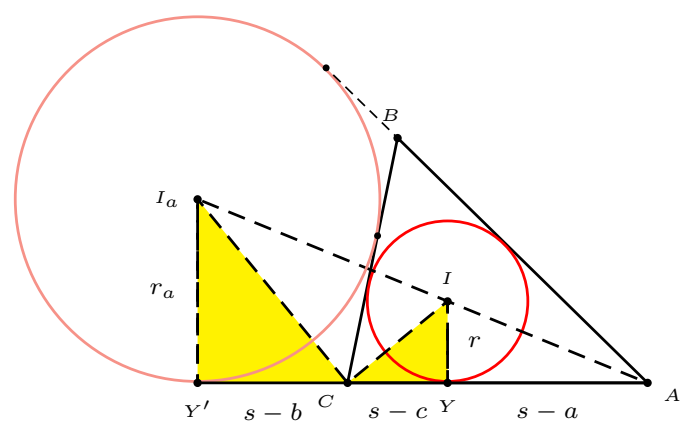

Proof. Consider the incircle and the excircle on the opposite side of $A$. From the similarity of triangles $A I Z$ and $A I^{\prime} Z^{\prime}$,

$$
\frac{r}{r_{a}}=\frac{s-a}{s}
$$

From the similarity of triangles $C I Y$ and $I^{\prime} C Y^{\prime}$,

$$
r \cdot r_{a}=(s-b)(s-c) \text {. }
$$

From these,

$$
r=\sqrt{\frac{(s-a)(s-b)(s-c)}{s}},
$$

and the area of the triangle is

$$
\triangle=r s=\sqrt{s(s-a)(s-b)(s-c)} .
$$




\subsection{Heron triangles}

A Heron triangle is one whose sidelengths and area are both integers. It can be constructed by joining two integer right triangles along a common leg. For example, by joining the two Pythagorean triangles $(9,12,15)$ and $(5,12,13)$, we obtain the Heron triangle $(13,14,15)$ with area 84 .

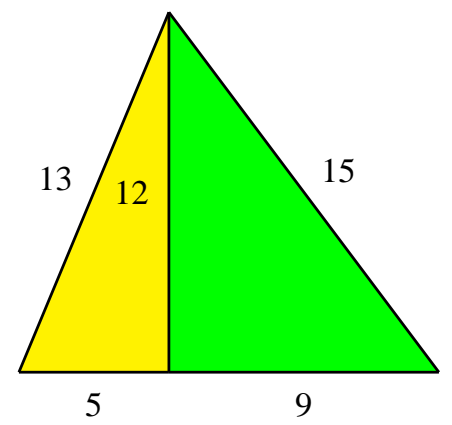

\section{Some properties of Heron triangles}

1. The semiperimeter is an integer.

2. The area is always a multiple of 6 .

\section{Exercise}

1. Construct four Heron triangles by joining suitable multiples of $(3,4,5)$ and $(5,12,13)$ along common legs. The Heron triangles you obtain should be primitive, i.e., the sidelengths of each should be relatively prime.

2. Can the Heron triangle $(25,34,39,420)$ be obtained by joining two Pythagorean triangles along a common leg? 


\subsection{Heron triangles with consecutive sides}

If $(b-1, b, b+1, \triangle)$ is a Heron triangle, then $b$ must be an even integer. We write $b=2 m$. Then $s=3 m$, and $\triangle^{2}=3 m^{2}(m-1)(m+1)$. This requires $m^{2}-1=3 k^{2}$ for an integer $k$, and $\triangle=3 \mathrm{~km}$. The solutions of $m^{2}-3 k^{2}=1$ can be arranged in a sequence

$$
\left(\begin{array}{c}
m_{n+1} \\
k_{n+1}
\end{array}\right)=\left(\begin{array}{ll}
2 & 3 \\
1 & 2
\end{array}\right)\left(\begin{array}{c}
m_{n} \\
k_{n}
\end{array}\right), \quad\left(\begin{array}{c}
m_{1} \\
k_{1}
\end{array}\right)=\left(\begin{array}{l}
2 \\
1
\end{array}\right) .
$$

From these, we obtain the side lengths and the area.

The middle sides form a sequence $\left(b_{n}\right)$ given by

$$
b_{n+2}=4 b_{n+1}-b_{n}, \quad b_{0}=2, b_{1}=4 .
$$

The areas of the triangles form a sequence

$$
\triangle_{n+2}=14 \triangle_{n+1}-\triangle_{n}, \quad T_{0}=0, T_{1}=6 .
$$

\begin{tabular}{|c|c|c|l|}
\hline$n$ & $b_{n}$ & $T_{n}$ & Heron triangle \\
\hline \hline 0 & 2 & 0 & $(1,2,3,0)$ \\
\hline 1 & 4 & 6 & $(3,4,5,6)$ \\
\hline 2 & 14 & 84 & $(13,14,15,84)$ \\
\hline 3 & & & \\
\hline 4 & & & \\
\hline 5 & & & \\
\hline
\end{tabular}

\section{Exercise}

1. There is a uniquely Heron triangle with sides $(b-1, b, b+1)$ in which $b$ is an 8-digit number. What is the area of this Heron triangle? 
Appendix: Heron triangles with sides $<100$

\begin{tabular}{|c|c|c|c|c|}
\hline$(a, b, c, \triangle)$ & $(a, b, c, \triangle)$ & $(a, b, c, \triangle)$ & $(a, b, c, \triangle)$ & $(a, b, c, \triangle)$ \\
\hline$\overline{(3,4,5,6)}$ & $\overline{\overline{(5,5,6,12)}}$ & $\overline{\overline{(5,5,8,12)}}$ & $\overline{(\overline{(5,12,13,30)}}$ & $(10,13,13,60)$ \\
\hline$(4,13,15,24)$ & $(13,14,15,84)$ & $(9,10,17,36)$ & $(8,15,17,60)$ & $(16,17,17,120)$ \\
\hline$(11,13,20,66)$ & $(7,15,20,42)$ & $(10,17,21,84)$ & $(13,20,21,126)$ & $(13,13,24,60)$ \\
\hline$(12,17,25,90)$ & $(7,24,25,84)$ & $(14,25,25,168)$ & $(3,25,26,36)$ & $(17,25,26,204)$ \\
\hline$(17,25,28,210)$ & $(20,21,29,210)$ & $(6,25,29,60)$ & $(17,17,30,120)$ & $(11,25,30,132)$ \\
\hline$(5,29,30,72)$ & $(8,29,35,84)$ & $(15,34,35,252)$ & $(25,29,36,360)$ & $(19,20,37,114)$ \\
\hline$(15,26,37,156)$ & $(13,30,37,180)$ & $(12,35,37,210)$ & $(24,37,37,420)$ & $(16,25,39,120)$ \\
\hline$(17,28,39,210)$ & $(25,34,39,420)$ & $(10,35,39,168)$ & $(29,29,40,420)$ & $(13,37,40,240)$ \\
\hline$(25,39,40,468)$ & $(15,28,41,126)$ & $(9,40,41,180)$ & $(17,40,41,336)$ & $(18,41,41,360)$ \\
\hline$(29,29,42,420)$ & $(15,37,44,264)$ & $(17,39,44,330)$ & $(13,40,45,252)$ & $(25,25,48,168)$ \\
\hline$(29,35,48,504)$ & $(21,41,50,420)$ & $(39,41,50,780)$ & $(26,35,51,420)$ & $(20,37,51,306)$ \\
\hline$(25,38,51,456)$ & $(13,40,51,156)$ & $(27,29,52,270)$ & $(25,33,52,330)$ & $(37,39,52,720)$ \\
\hline$(15,41,52,234)$ & $(5,51,52,126)$ & $(25,51,52,624)$ & $(24,35,53,336)$ & $(28,45,53,630)$ \\
\hline$(4,51,53,90)$ & $(51,52,53,1170)$ & $(26,51,55,660)$ & $(20,53,55,528)$ & $(25,39,56,420)$ \\
\hline$(53,53,56,1260)$ & $(33,41,58,660)$ & $(41,51,58,1020)$ & $(17,55,60,462)$ & $(15,52,61,336)$ \\
\hline$(11,60,61,330)$ & $(22,61,61,660)$ & $(25,52,63,630)$ & $(33,34,65,264)$ & $(20,51,65,408)$ \\
\hline$(12,55,65,198)$ & $(33,56,65,924)$ & $(14,61,65,420)$ & $(36,61,65,1080)$ & $(16,63,65,504)$ \\
\hline$(32,65,65,1008)$ & $(35,53,66,924)$ & $(65,65,66,1848)$ & $(21,61,68,630)$ & $(43,61,68,1290)$ \\
\hline$(7,65,68,210)$ & $(29,65,68,936)$ & $(57,65,68,1710)$ & $(29,52,69,690)$ & $(37,37,70,420)$ \\
\hline$(9,65,70,252)$ & $(41,50,73,984)$ & $(26,51,73,420)$ & $(35,52,73,840)$ & $(48,55,73,1320)$ \\
\hline$(19,60,73,456)$ & $(50,69,73,1656)$ & $(25,51,74,300)$ & $(25,63,74,756)$ & $(35,44,75,462)$ \\
\hline$(29,52,75,546)$ & $(32,53,75,720)$ & $(34,61,75,1020)$ & $(56,61,75,1680)$ & $(13,68,75,390)$ \\
\hline$(52,73,75,1800)$ & $(40,51,77,924)$ & $(25,74,77,924)$ & $(68,75,77,2310)$ & $(41,41,80,360)$ \\
\hline$(17,65,80,288)$ & $(9,73,80,216)$ & $(39,55,82,924)$ & $(35,65,82,1092)$ & $(33,58,85,660)$ \\
\hline$(29,60,85,522)$ & $(39,62,85,1116)$ & $(41,66,85,1320)$ & $(36,77,85,1386)$ & $(13,84,85,546)$ \\
\hline$(41,84,85,1680)$ & $(26,85,85,1092)$ & $(72,85,85,2772)$ & $(34,55,87,396)$ & $(52,61,87,1560)$ \\
\hline$(38,65,87,1140)$ & $(44,65,87,1386)$ & $(31,68,87,930)$ & $(61,74,87,2220)$ & $(65,76,87,2394)$ \\
\hline$(53,75,88,1980)$ & $(65,87,88,2640)$ & $(41,50,89,420)$ & $(28,65,89,546)$ & $(39,80,89,1560)$ \\
\hline$(21,82,89,840)$ & $(57,82,89,2280)$ & $(78,89,89,3120)$ & $(53,53,90,1260)$ & $(17,89,90,756)$ \\
\hline$(37,72,91,1260)$ & $(60,73,91,2184)$ & $(26,75,91,840)$ & $(22,85,91,924)$ & $(48,85,91,2016)$ \\
\hline$(29,75,92,966)$ & $(39,85,92,1656)$ & $(34,65,93,744)$ & $(39,58,95,456)$ & $(41,60,95,798)$ \\
\hline$(68,87,95,2850)$ & $(73,73,96,2640)$ & $(37,91,96,1680)$ & $(51,52,97,840)$ & $(65,72,97,2340)$ \\
\hline$(26,73,97,420)$ & $(44,75,97,1584)$ & $(35,78,97,1260)$ & $(75,86,97,3096)$ & $(11,90,97,396)$ \\
\hline$(78,95,97,3420)$ & & & & \\
\hline
\end{tabular}




\section{Appendix: A famous unsolved problem}

Find a Heron triangle whose medians have integer lengths. This is a famous unsolved problem. See [Guy, Problem D21]. The triangle with sides 136, 170, 174 have medians 158, 131, 127. But it is not a Heron triangle. It has an area

Buchholz and Rathbun have found an infinite set of Heron triangles with two integer medians. Here is the first one.

Let $a=52, b=102$, and $c=146$.

Verify that this triangle is Heron and find the lengths of the medians. 


\section{Project: Triangles whose sides and one altitude are in arithmetic progression}

This is an extension of Problem 29 of Isaac Newton's Lectures on Algebra ([Whiteside, pp.234 - 237]).

(A) Newton considered a triangle $A B C$ with an altitude $D C$. Clearly, $D C$ is shorter than $A C$ and $B C$. Setting $A C=a, B C=x, D C=$ $2 x-a$, and $A B=2 a-x$, he obtained

$$
16 x^{4}-80 a x^{3}+144 a^{2} x^{2}-10 a^{3} x+25 a^{4}=0 .
$$

"Divide this equation by $2 x-a$ and there will result $8 x^{3}-36 a x^{2}+$ $54 a^{2} x-25 a^{3}=0 "$. Newton did not solve this equation nor did he give any numerical example. Actually, $(\dagger)$ can be rewritten as

$$
(2 x-3 a)^{3}+2 a^{3}=0,
$$

so that $x=\frac{a}{2}(3-\sqrt[3]{2})$, the other two roots being complex. By taking $a=2$, we may assume the sides of the triangles to be

and the altitude on the longest side to be

The angles of the triangles are

(B) Recalling the Heron triangle with sides 13, 14, 15 with altitude 12 on the side 14, we realize that these lengths can be in A.P. in some other order. Note that the altitude in question is either the first or the second terms of the A.P. (in increasing order). Assuming unit length for this altitude, and $x>0$ for the common difference, we have either

1. the three sides of the triangles are $1+x, 1+2 x$, and $1+3 x$, or

2. the sides of the triangles are $1-x, 1+x$, and $1+2 x$, and the altitude on the shortest side is 1 .

In (1), the area of the triangle, by the Heron formula, is given by

$$
\triangle^{2}=\frac{3}{16}(1+2 x)^{2}(1+4 x)
$$

On the other hand, $\triangle=\frac{1}{2} \cdot 1 \cdot(1+k x)$ for $k=1,2,3$. These lead to the equations 
- for $k=1: 48 x^{3}+56 x^{2}+16 x-1=0$,

- for $k=2: 48 x^{3}+44 x^{2}+8 x-1=0$,

- for $k=3: 48 x^{3}+24 x-1=0$.

The case $k=3$ has been dealt with in Newton's solution.

For $k=2$, the polynomial factors as so that we have $x=$ . This leads to the Heronian triangle with sides $13,14,15$, and altitude 12 on the side 14 . The angles of the triangles are

For $k=1$, it is easy to see, using elementary calculus, that the polynomial $48 x^{3}+56 x^{2}+16 x-1$ has exactly one real root, which is positive.

This gives a similarity class of triangle with the three sides and the altitude on the shortest side in A.P. More detailed calculation shows that the angles of such triangles are

Now we consider (2), when the altitude in question is the second term of the A.P. Instead of constructing an equation in $x$, we seek one such triangle with sides $15,17+2 z, 18+3 z$, and the altitude $16+z$ on the shortest side. By considering the area of the triangle in two different ways, we obtain the cubic equation

$$
z^{3}-120 z+16=0 .
$$

This can be solved by writing $z=4 \sqrt{10} \sin \theta$ for an angle $\theta$. Using the trigonometric identity $\sin 3 \theta=3 \sin \theta-4 \sin ^{3} \theta$, we reduce this to

$$
\sin 3 \theta=
$$

so that the positive roots of $(*)$ are the two numbers

$$
z=
$$

We obtain two similarity classes of triangles, respectively with angles

and

There are altogether five similarity classes of triangles whose three sides and one altitude, in some order, are in arithmetic progression. 


\section{Chapter 10}

\section{The golden section}

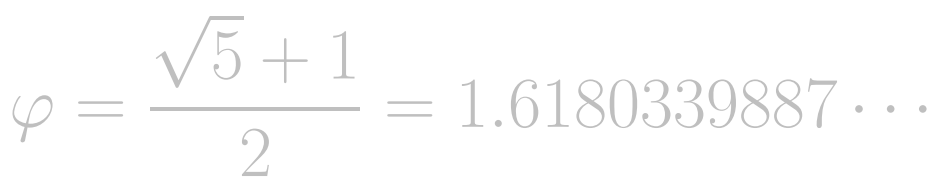

1 The golden triangle

2 Dissection of a square

3 Dissection of a rectangle

4 Characterizations of the golden triangle

5 What is the most non-isosceles triangle?

Appendix: Cutting a square into three similar parts Exercise

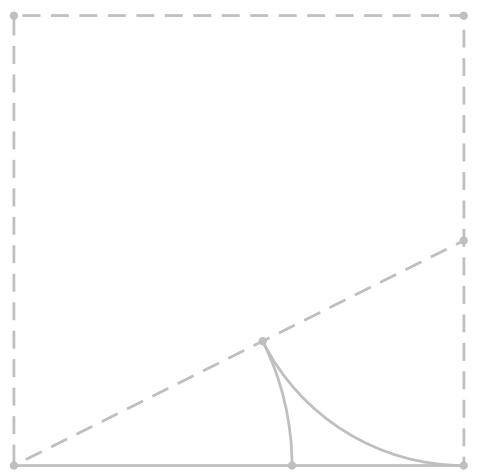




\subsection{The golden section $\varphi$}

A segment $A B$ is said to be divided into the golden ratio by a point $P$ if $A P^{2}=A B \cdot P B$.
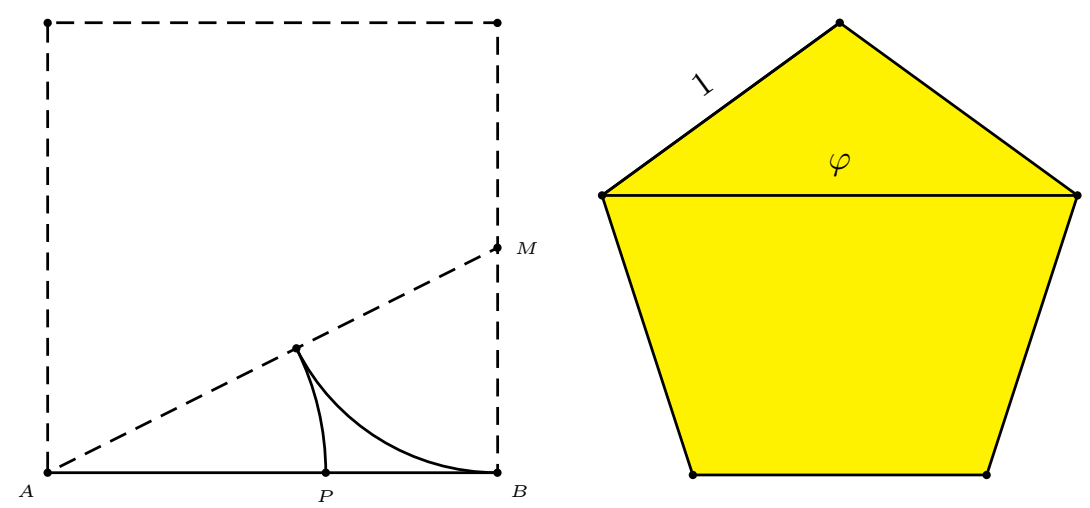

$$
\frac{A B}{A P}=\varphi=\frac{\sqrt{5}+1}{2}
$$

\section{Some basic properties of $\varphi$}

1. $\varphi^{2}=1+\varphi ; \frac{1}{\varphi}=\varphi-1$.

2. The diagonal and the side of a regular pentagon are in the golden ratio.

3. The ratio of successive Fibonacci numbers tends to $\varphi$.

4. The simple continued fraction expansion of $\varphi$ is

$$
1+\frac{1}{1+\frac{1}{1+\frac{1}{1+\frac{1}{\ddots}}}}
$$




\subsection{Dissection of a square}

Show how the square should be dissected so that it can be reassembled into a rectangle.
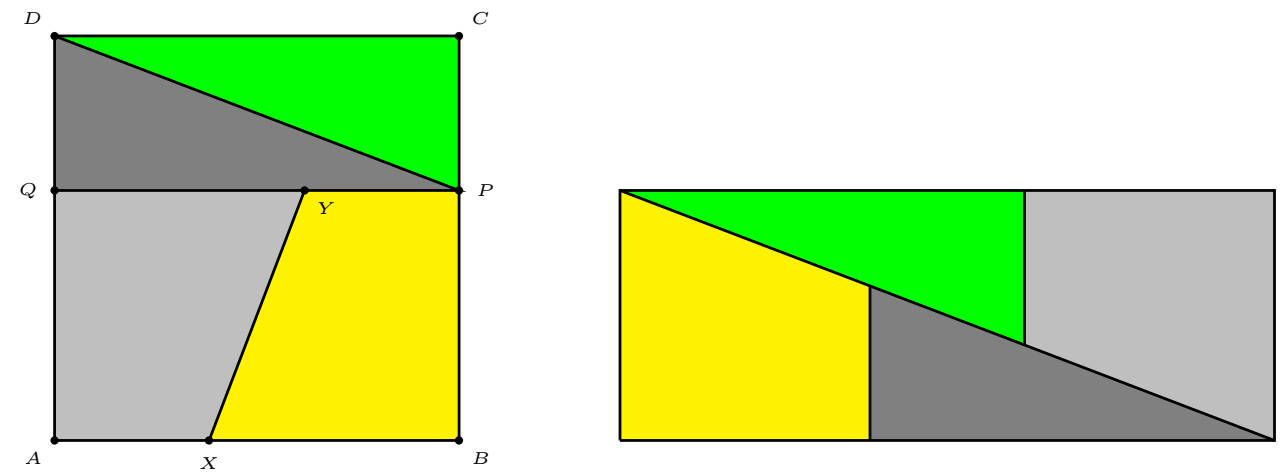

Let $A B=B C=a$. We want to find $x=A X=P C$ such that the areas of the rectangle and the square are the same. 
Resolve the paradox: How can the area increase after reassembling?
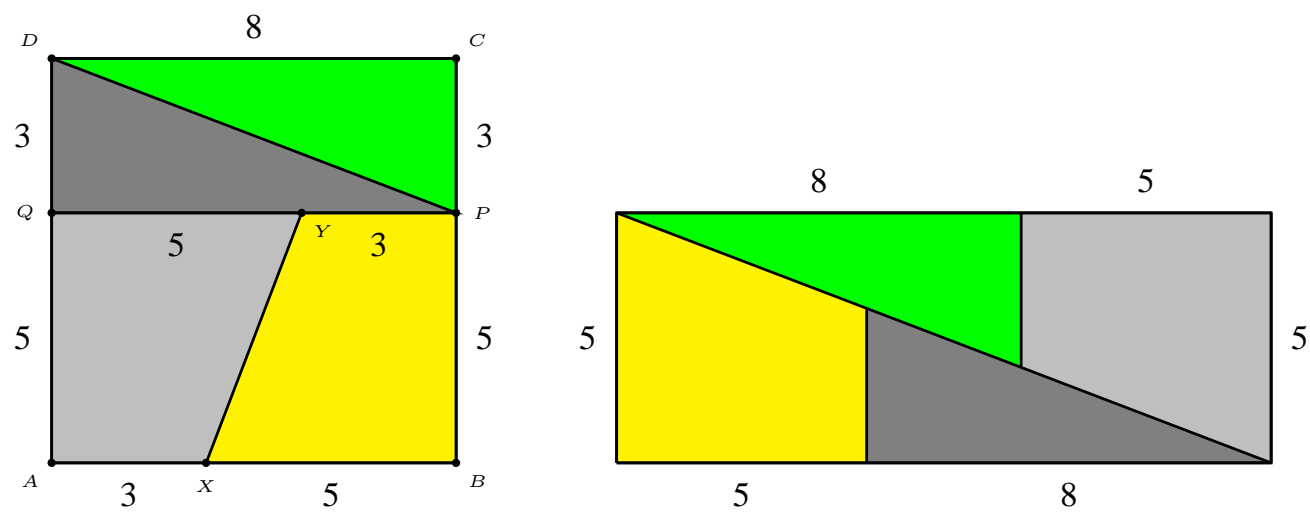


\subsection{Dissection of a rectangle}

Let $P$ and $Q$ be points on the sides $A B$ and $B C$ of rectangle $A B C D$.

If the areas of triangles $A P D, B P Q$ and $C D Q$ are equal, then $P$ and $Q$ divide the sides in the golden ratio.

If, in addition, $D P=P Q$, then the rectangle is golden, and $\angle D P Q$ is a right angle.

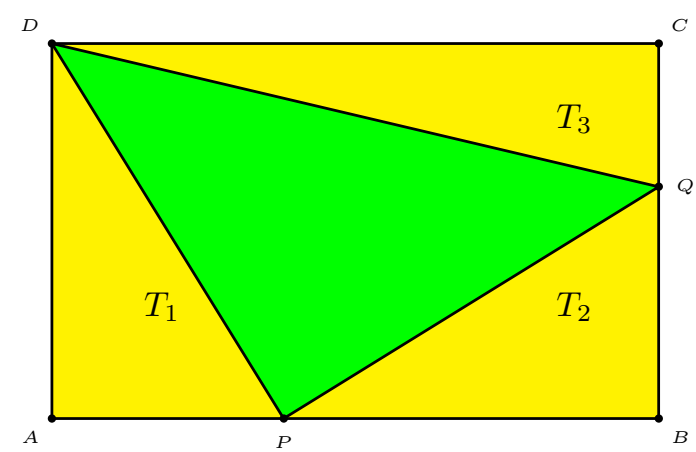




\subsection{The golden right triangle}

The golden right triangle is the one whose hypotenuse and one leg are in the golden ratio.

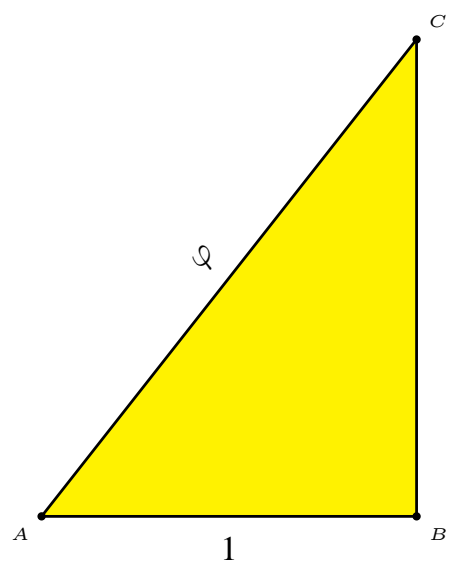

\section{Characterizations of the golden triangle}

1. If the sides of a right triangle are in geometric progression, then it is the golden triangle.

2. If the altitude of a right triangle divides the hypotenuse in the golden ratio, then the triangle is golden.

3. If one side of a right triangle is the geometric mean of the other two sides, then this is a golden right triangle.

4. If the sides of a right triangle are respectively the harmonic, geometric, and arithmetic means of two numbers, then the triangle is a golden right triangle. 
Find the isosceles triangle of smallest perimeter that circumscribed a given semicircle.

Suppose the semicircle has radius 1 . If $\theta$ is the base angle, the perimeter is $2(\tan \theta+\cot \theta+\csc \theta)$. We want to find the $\theta$ that minimizes this perimeter.

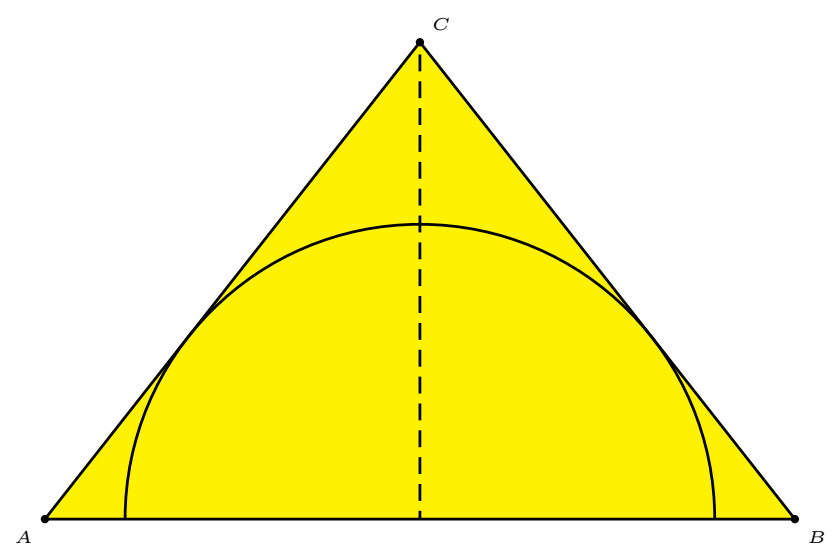




\subsection{What is the most non-isosceles triangle?}

Given a triangle, there are 6 ratios obtained by comparing the lengths of a pair of sides. The one which is closest to 1 is called the ratio of non-isoscelity of the triangle. Determine the range of the ratio of nonisoscelity of triangles.

Theorem 10.1. A number $\eta$ is the ratio of non-isoscelity of a triangle if and only if it lies in the interval $\left(\frac{1}{\varphi}, 1\right]$.

Proof. First note that if $r<1$ is the ratio of the length of two sides of a triangle, then so is $\frac{1}{r}$. Since $\frac{1}{2}\left(r+\frac{1}{r}\right)>1, r$ is closer to 1 than $\frac{1}{r}$. It follows that the ratio of non-isoscelity $\eta \leq 1$.

If $a \leq b \leq c$ are the side lengths, then $\eta=\max \left(\frac{a}{b}, \frac{b}{c}\right)$. Since $a+b>$ $c$, we have

$$
\eta+1 \geq \frac{a}{b}+1>\frac{c}{b} \geq \frac{1}{\eta} .
$$

It follows that $\eta^{2}+\eta>1$. Since the roots of $x^{2}+x-1=0$ are $\frac{1}{\varphi}>0$ and $-\varphi<0$, we must have $\eta>\varphi-1$. This shows that $\eta \in\left(\frac{1}{\varphi}, 1\right]$.

For each number $t$ in this range, the triangle with sides $t, 1, \frac{1}{t}$ is one with ratio of non-isoscelity $t$.

There is therefore no "most non-isosceles" triangle. Instead, the most non-isosceles triangles almost degenerate to a segment divided in the golden ratio. 


\section{Project: Cutting a square into three similar parts}

Cut a square into three similar parts, no two congruent.

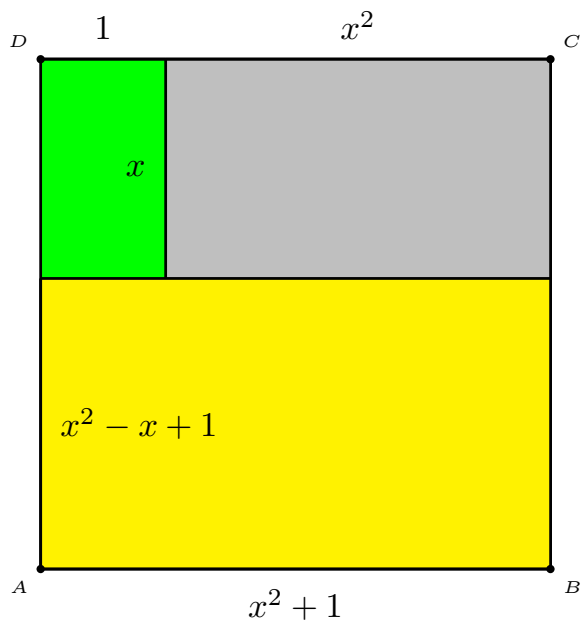




\section{Exercise}

1. Show that $\cos 36^{\circ}=\frac{\varphi}{2}$.

2. Which of the two equilateral triangles inscribed in a regular pentagon has larger area?
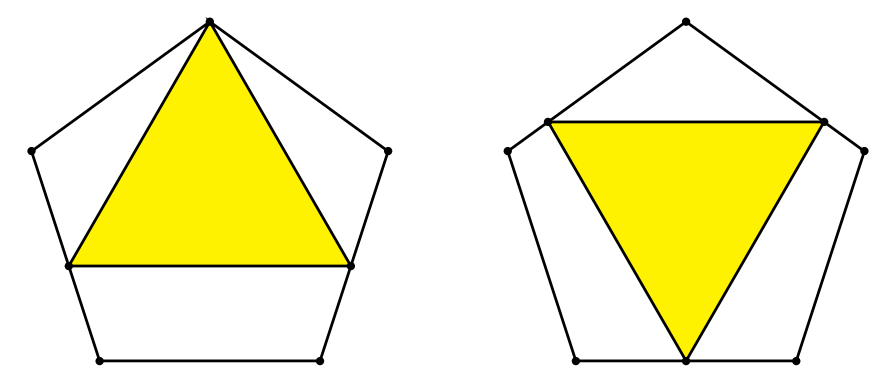

3. Which of the two squares inscribed in a regular pentagon has larger area?
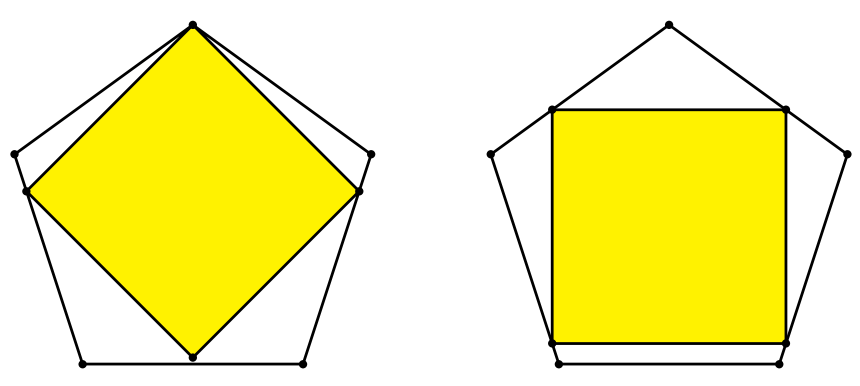
If we assume the regular pentagon inscribed in a unit circle, the sidelengths of these squares are respectively

$$
\frac{\sqrt{10}}{8}(\sqrt{5}+1)
$$

and

$$
\frac{\sqrt{5}}{2}(\sqrt{10-2 \sqrt{5}}-\sqrt{5}+1) .
$$

The first square exceeds the second one by

$$
\frac{1}{8}(20+5 \sqrt{2}+\sqrt{10}-4 \sqrt{5}-4 \sqrt{50-10 \sqrt{5}}) \approx 0.0324786 \cdots
$$

One of you [GC] suggested an "easy" construction of the square as follows.
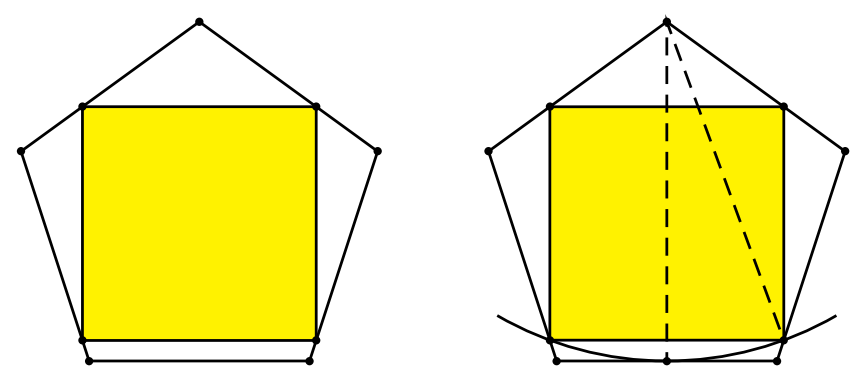

This indeed is not exact, but it gives an approximate rectangle with base

$$
\frac{1}{2} \sqrt{5(2-2 \sqrt[4]{5}+\sqrt{5})}
$$

which is smaller only by $0.08 \%$ !

As for the triangles, the first one has length $\sqrt{\frac{5}{2}(4+\sqrt{5}-\sqrt{15+6 \sqrt{5}})}$, and the second one has shorter length $\frac{\sqrt{10}(\sqrt{5}+1)}{(\sqrt{5}-1) \sqrt{5-\sqrt{5}}+2 \sqrt{6}}$. The difference between the two is approx $0.0328 \cdots$. 


\section{Chapter 11}

\section{Constructions with the golden section}

1 Construction of golden rectangle

2 Hofstetter's compass-only construction of the golden section

3 Hofstetter's 5-step division of a segment in the golden section

4 Construction of a regular pentagon

5 Ahlburg's parsimonious construction of the regular pentagon

6 Construction of a regular 17-gon

Exercise

Project

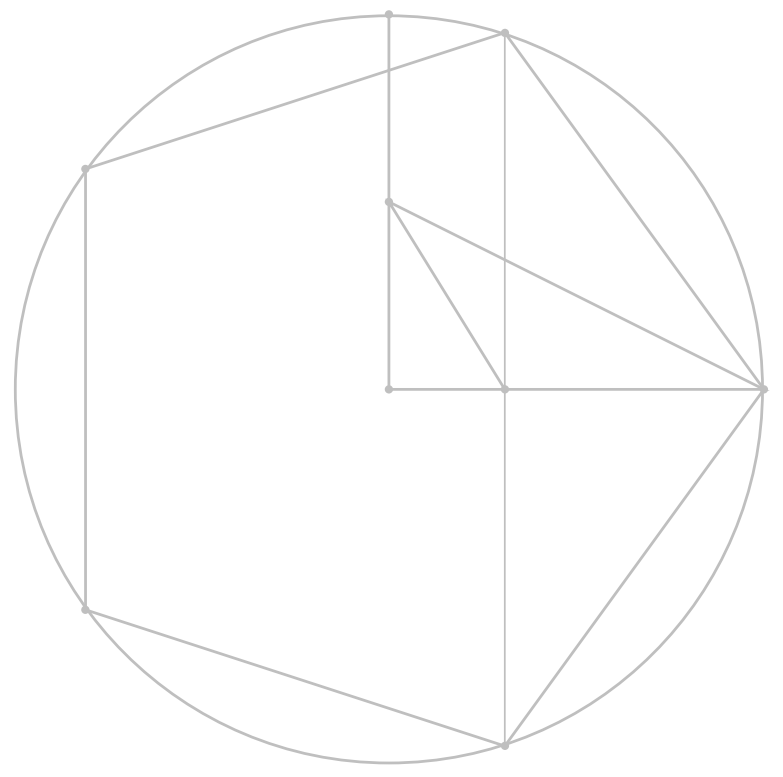




\subsection{Construction of golden rectangle}

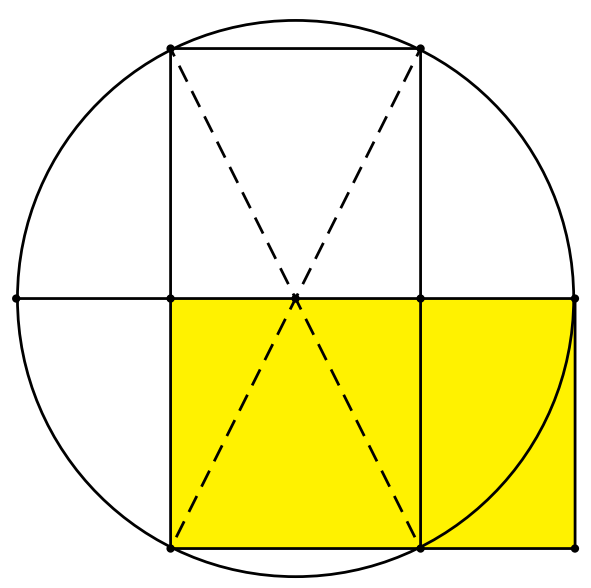




\subsection{Hofstetter's compass-only construction of the golden section}

Kurt Hofstetter has found the following construction of the golden section by striking the compass only five times. ${ }^{1}$

We denote by $P(Q)$ the circle with $P$ as center and $P Q$ as radius. Figure 1 shows two circles $A(B)$ and $B(A)$ intersecting at $C$ and $D$. The line $A B$ intersects the circles again at $E$ and $F$. The circles $A(F)$ and $B(E)$ intersect at two points $X$ and $Y$. It is clear that $C, D, X$, $Y$ are on a line. It is much more interesting to note that $D$ divides the segment $C X$ in the golden ratio, i.e.,

$$
\frac{C D}{C X}=\frac{\sqrt{5}-1}{2} .
$$

This is easy to verify. If we assume $A B$ of length 2 , then $C D=2 \sqrt{3}$ and $C X=\sqrt{15}+\sqrt{3}$. From these,

$$
\frac{C D}{C X}=\frac{2 \sqrt{3}}{\sqrt{15}+\sqrt{3}}=\frac{2}{\sqrt{5}+1}=\frac{\sqrt{5}-1}{2} .
$$
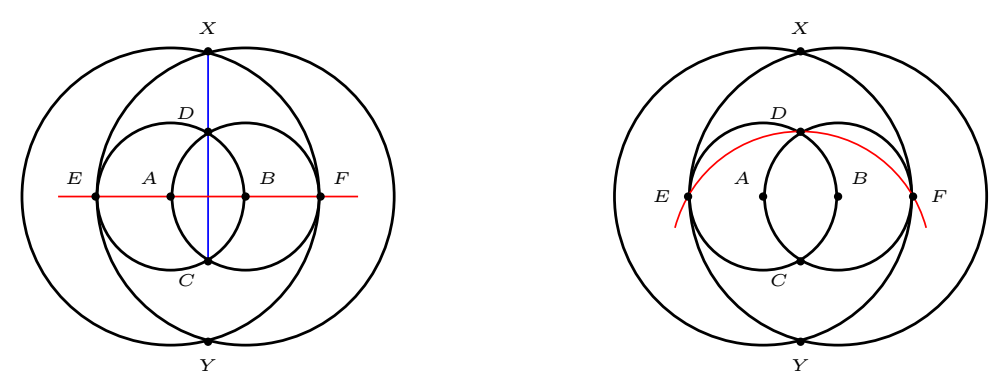

This shows that to construct three collinear points in golden section, we need four circles and one line. It is possible, however, to replace the line $A B$ by a circle, say $C(D)$. See Figure 2. Thus, the golden section can be constructed with compass only, in 5 steps.

Here is a simple application: to divide a given segment into golden section. $^{2}$

Construction 11.1. Given a segment $A B$, construct

\footnotetext{
${ }^{1}$ K. Hofstetter, A simple construction of the golden section, Forum Geometricorum, 2 (2002) 65-66.

${ }^{2}$ Communicated by K. Hofstetter on December 9, 2003.
} 
1. $\mathrm{e}_{1}=A(B)$,

2. $\mathrm{e}_{2}=B(A)$, intersecting $\mathcal{C}_{1}$ at $C$ and $D$,

3. $C D$ to intersect $A B$ at (their common midpoint) $M$,

4. $\mathrm{C}_{3}=A(M)$ to intersect $\mathrm{C}_{2}$ at $E$,

5. $\mathrm{C}_{4}=E(A)$ to intersect $\mathcal{C}_{3}$ at $F$ and $F^{\prime}, F$ closer to $M$ then $G^{\prime}$,

6. $E F$ and extend to intersect $A B$ at $G$.

The point $G$ divides the segment $A B$ in the golden section.

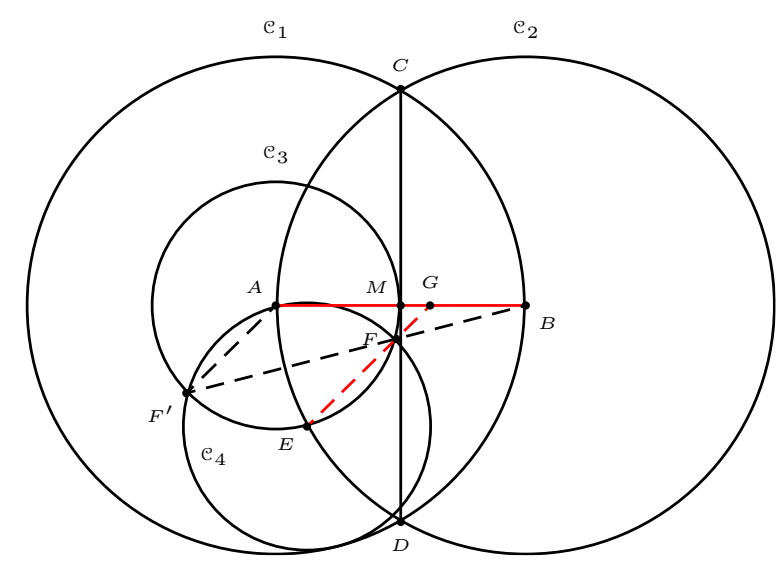

Proof. By [1], $F$ divides $F^{\prime} B$ in the golden section. Since $E F$ is parallel to $F^{\prime} A, G$ divides $A B$ in the golden section as well.

Remark. If the lines $E F^{\prime}$ and $A B$ intersect at $G^{\prime}$, then $A$ divides $G^{\prime} B$ in the golden section. 


\subsection{Hofstetter's 5-step division of a segment in the golden section}

K. Hofstetter has also found the following parsimonious division of a given segment in the golden section. ${ }^{3}$

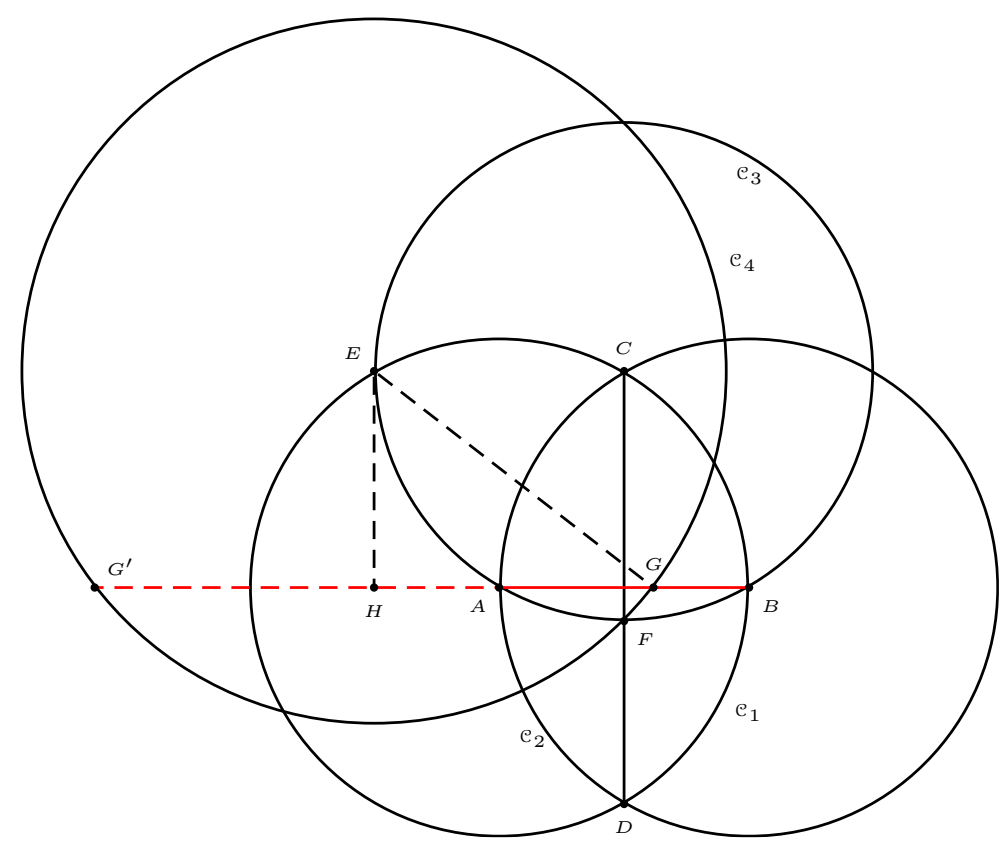

Construction 11.2. Given a segment $A B$, construct

1. $\mathrm{C}_{1}=A(B)$,

2. $\mathrm{C}_{2}=B(A)$, intersecting $\mathrm{C}_{1}$ at $C$ and $D$,

3. $\mathrm{C}_{3}=C(A)$, intersecting $\mathrm{e}_{1}$ again at $E$,

4. the segment $C D$ to intersect $\mathrm{C}_{3}$ at $F$,

5. $\mathrm{C}_{4}=E(F)$ to intersect $A B$ at $G$.

The point $G$ divides the segment $A B$ in the golden section.

\footnotetext{
${ }^{3}$ K. Hofstetter, A 5-step division of a segment in the golden section, Forum Geom., 3 (2003) 205-206. After the publication of this paper, it was found that the same construction has been given previously by E. Lemoine, Géométrographie ou art des Constructions géométriques, C. Naud, Paris, 1902, p.51, and J. Reusch, Planimetrische Konstruktionen in geometrographischer Ausfuhrung, (Teubner 1904), pg 37.
} 
Proof. Suppose $A B$ has unit length. Then $C D=\sqrt{3}$ and $E G=E F=$ $\sqrt{2}$. Let $H$ be the orthogonal projection of $E$ on the line $A B$. Since $H A=\frac{1}{2}$, and $H G^{2}=E G^{2}-E H^{2}=2-\frac{3}{4}=\frac{5}{4}$, we have $A G=$ $H G-H A=\frac{1}{2}(\sqrt{5}-1)$. This shows that $G$ divides $A B$ in the golden section.

Remark. The other intersection $G^{\prime}$ of $\mathcal{C}_{4}$ and the line $A B$ is such that $G^{\prime} A: A B=\frac{1}{2}(\sqrt{5}+1): 1$. 


\subsection{Construction of regular pentagon}

Let $O A$ and $O Y$ be two perpendicular radii of a circle, center $O$.

1. Mark the midpoint $M$ of $O Y$ and bisect angle $O M A$ to intersect $O A$ at $P$.

2. Construct the perpendicular to $O A$ at $P$ to intersect the circle at $B$ and $E$.

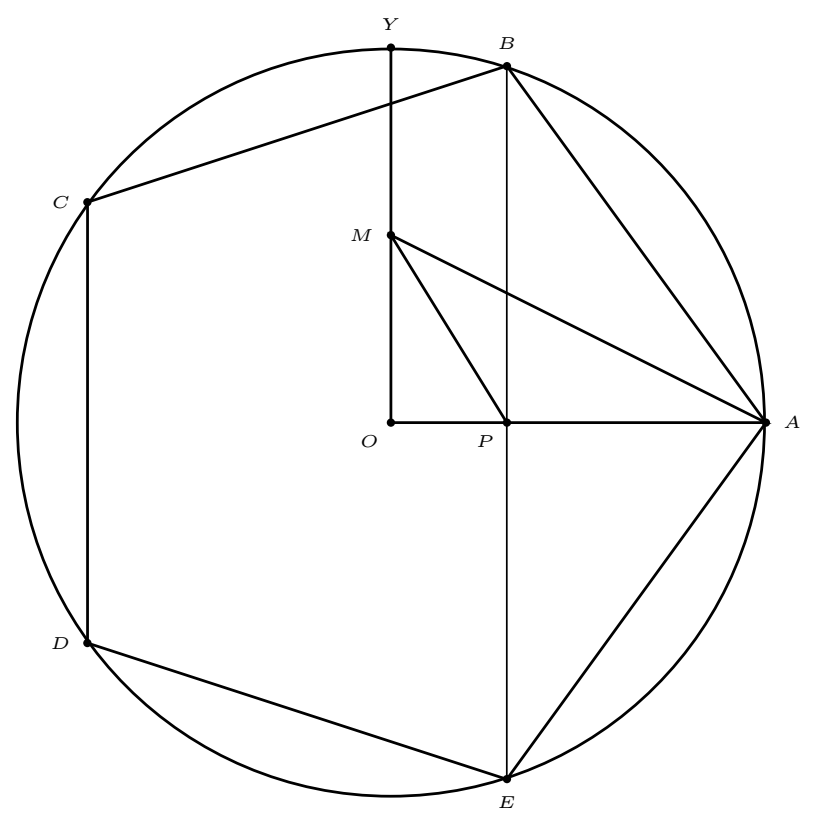

Then $A, B, E$ are three adjacent vertices of a regular pentagon inscribed in the circle. The remaining two vertices can be easily constructed. 


\subsection{Ahlburg's parsimonious construction of the regu- lar pentagon}

Make use of a given right triangle $A B^{\prime} C$ with $A C=2 B^{\prime} C$ to construct a regular pentagon in the fewest number of euclidean operations. (Euclidean operations include (i) setting a compass, (ii) striking an arc, (iii) drawing a line.

Between the side $a$ and the diagonal $d$ of a regular pentagon, there is the relation $a=\frac{\sqrt{5}-1}{2} d$. Here is Hayo Ahlburg's construction. ${ }^{4}$

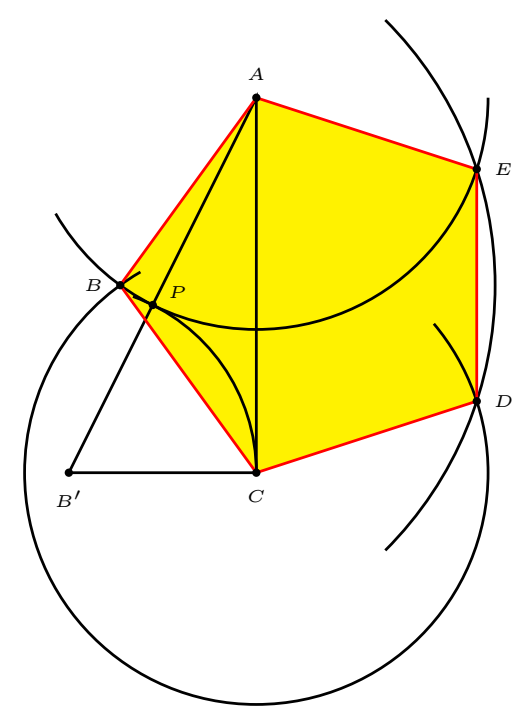

Construction 11.3. (1) Strike an $\operatorname{arc} B^{\prime}\left(B^{\prime} C\right)$, that is, with center $B^{\prime}$ and radius $B^{\prime} C$, meeting $A B^{\prime}$ at $P$.

(2) Strike an arc $A(A P)$.

(3) Strike an arc $C(A P)$, meeting $\operatorname{arc} A(A P)$ at $B$. E.

(4) Strike an arc $B(C A)$, meeting $\operatorname{arcs} A(A P)$ and $C(A P)$ at $D$ and

Then $A B C D E$ is the required regular pentagon. The construction requires 3 compass settings, striking 4 arcs, and drawing 5 lines for the sides of the pentagon, 12 euclidean construction in all.

${ }^{4}$ Crux Math., 6 (1980) 50. 


\subsection{Construction of a regular 17-gon}

To construct two vertices of the regular 17-gon inscribed in a given circle $O(A)$.

1. On the radius $O B$ perpendicular to $O A$, mark a point $J$ such that $O J=\frac{1}{4} O A$.

2. Mark a point $E$ on the segment $O A$ such that $\angle O J E=\frac{1}{4} \angle O J A$.

3. Mark a point $F$ on the diameter through $A$ such that $O$ is between $E$ and $F$ and $\angle E J F=45^{\circ}$.

4. With $A F$ as diameter, construct a circle intersecting the radius $O B$ at $K$.

5. Mark the intersections of the circle $E(K)$ with the diameter of $O(A)$ through $A$. Label the one between $O$ and $A$ points $P_{4}$, and the other and $P_{6}$.

6. Construct the perpendicular through $P_{4}$ and $P_{6}$ to intersect the circle $O(A)$ at $A_{4}$ and $A_{6} .{ }^{5}$

Then $A_{4}, A_{6}$ are two vertices of a regular 17-gon inscribed in $O(A)$. The polygon can be completed by successively laying off arcs equal to $A_{4} A_{6}$, leading to $A_{8}, A_{10}, \ldots, A_{16}, A_{1}=A, A_{3}, A_{5}, \ldots, A_{15}, A_{17}, A_{2}$.

\footnotetext{
${ }^{5}$ Note that $P_{4}$ is not the midpoint of $A F$.
} 


\section{Exercise}

1. Let $A B C$ be an equilateral triangle. The line joining the midpoints $D, E$ of two sides intersects the circumcircle at $F$. Then $E$ divides $D F$ in the golden section, i.e.,

$$
\frac{D E}{D F}=\frac{\sqrt{5}-1}{2}
$$

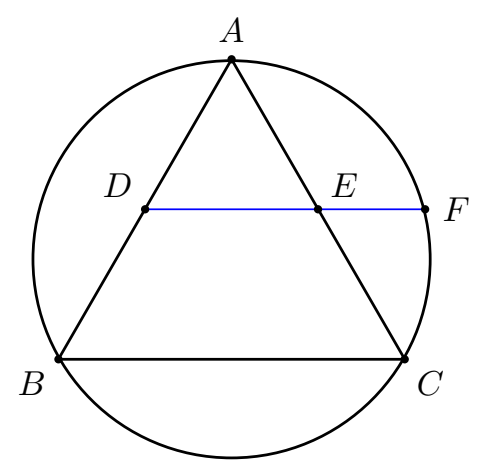

2. $M$ is the midpoint of the side $A B$ of a square $A B C D$. The line $D M$ intersects the circle with diameter $A B$ at two points, $P$ inside and $Q$ outside the square. Show that the rectangle $A P B Q$ is a golden rectangle, i.e., $P B: P A=(\sqrt{5}+1): 2$.

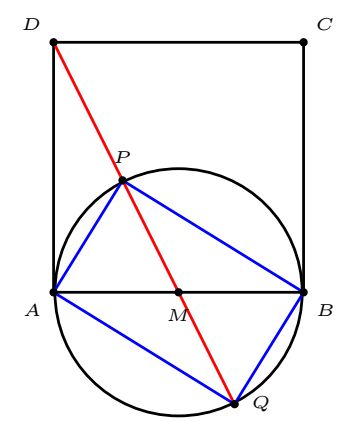




\section{Project}

This is a classic problem you should know if you teach geometry. While it is not difficult to guess what the answer is, it has been quite baffling to most people to arrive at the answer logically.

$A B C$ is an isosceles triangle with angle $A=20^{\circ} . E$ and $F$ are points on $A C$ and $A B$ such that $\angle E B C=60^{\circ}$ and $\angle F C B=50^{\circ}$. Calculate $\angle B E F$.

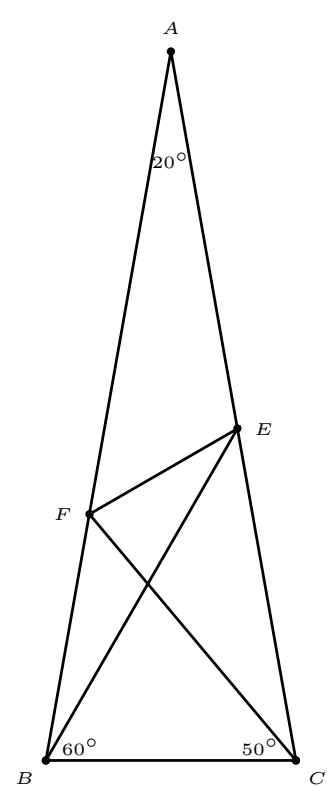




\section{Appendix: Another construction of regular pentagon ${ }^{6}$}

Given a circle, center $O$, let

(i) $A X Y$ be an isosceles triangle whose height is $\frac{5}{4}$ of the radius of the circle,

(ii) $B^{\prime}, C^{\prime}$ be points on the circumference such that $A X^{\prime}=A Y^{\prime}=$ radius, (iii) $P=A X \cap O B^{\prime}$ and $Q=A Y \cap O C^{\prime}$,

(iv) the line $P Q$ intersect the circumference at $B$ and $C$.

Then $A B$ and $A C$ are two sides of a regular pentagon inscribed in the circle.

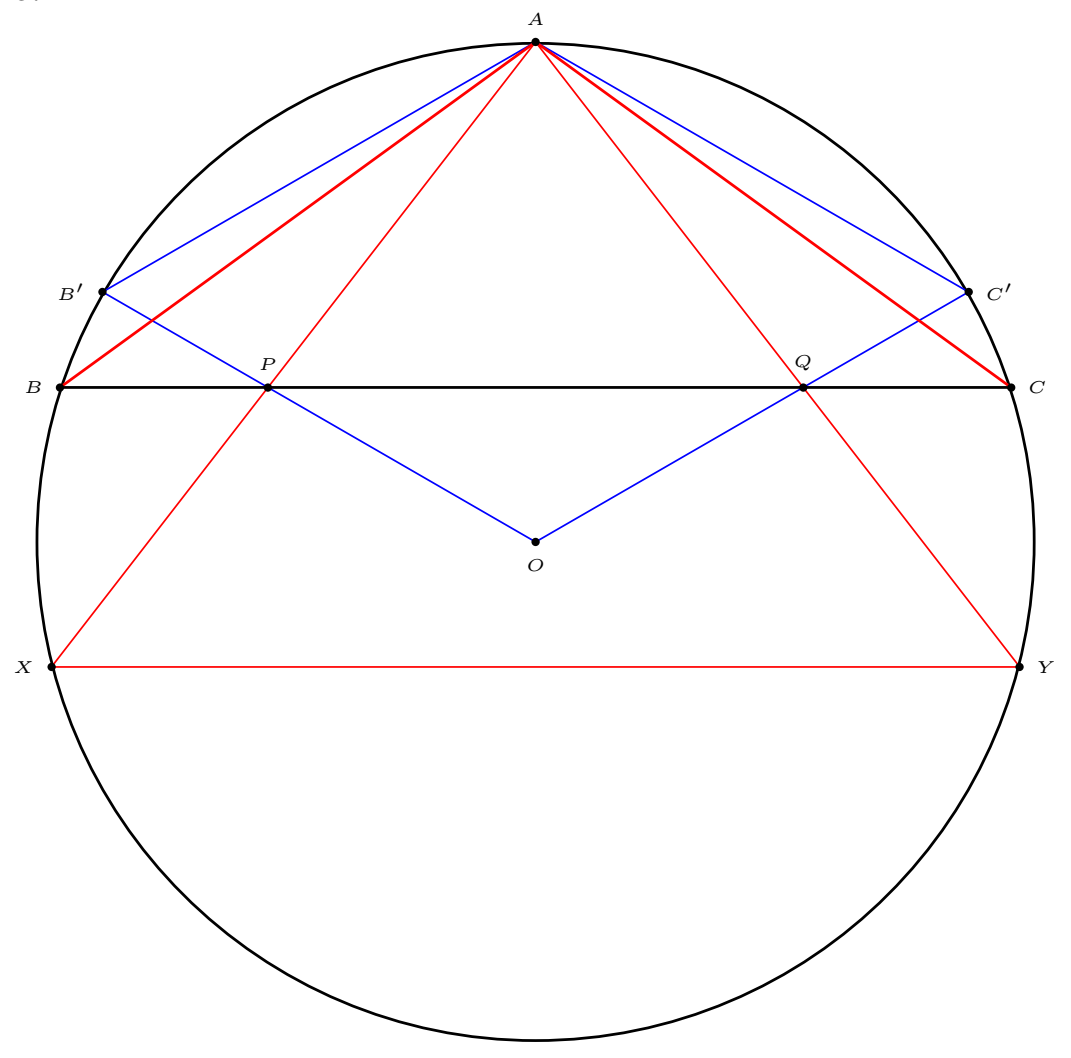

${ }^{6}$ D. Nelson, A regular pentagon construction, Math. Gazette, 61 (1977) 215-216. 
$A B C D E$ is a pentagon symmetrically inscribed in a circle such that $A B=C D=A E=$ the radius of the circle. $P$ and $Q$ are the midpoints of the sides $B C$ and $D E$. Show that triangle $A P Q$ is equilateral.

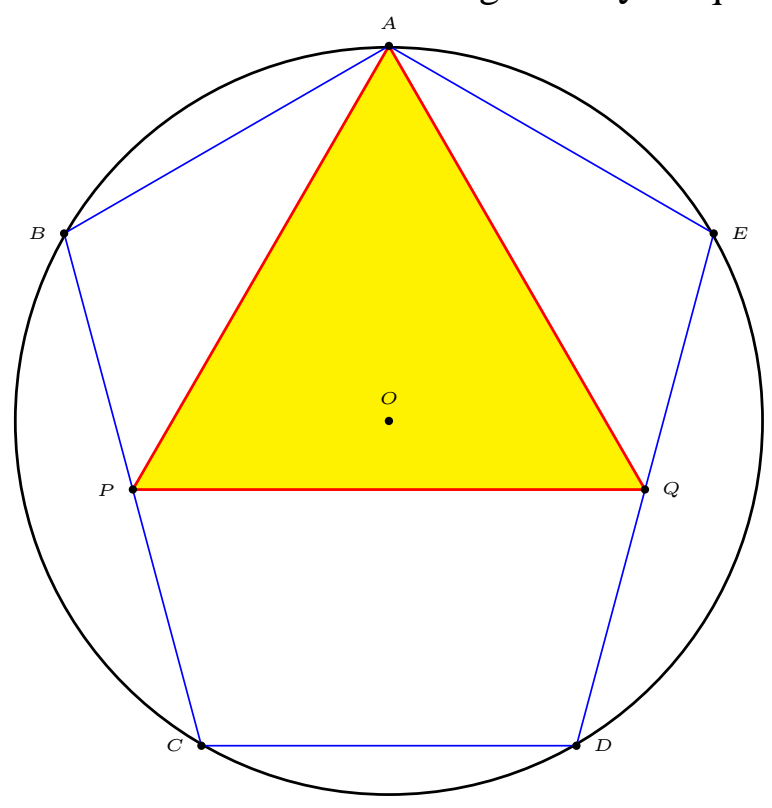




\title{
Chapter 12
}

\section{Cheney's card trick}

\author{
1 Principles \\ 2 Examples \\ Exercise
}

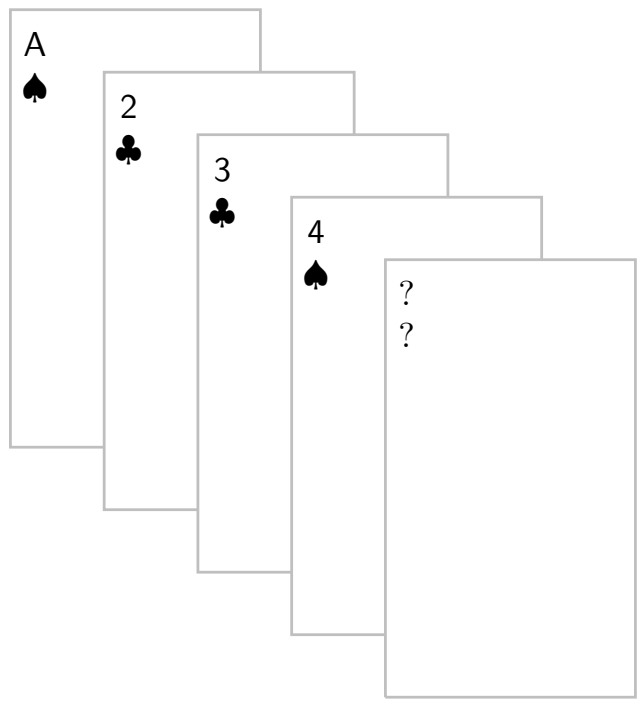




\subsection{Principles}

You are the magician's assistant. What he will do is to ask a spectator to give you any 5 cards from a deck of 52. You show him 4 of the cards, and in no time, he will tell everybody what the 5 th card is. This of course depends on you, and you have to do things properly by making use of the following three basic principles.

1. The pigeonhole principle. Among 5 cards at least 2 must be of the same suit. So you and the magician agree that the secret card has the same suit as the first card.

2. The distance of two points on a 13-hour clock is no more than 6 .

We decide which of the two cards to be shown as the first, and which to be kept secret. For calculations, we treat $A, J, Q$, and $K$ are respectively $1,11,12$, and 13 respectively.

Now you can determine the distance between these two cards. From one of these, going clockwise, you get to the other by travelling this distance on the 13-hour clock. Keep the latter as the secret card. Here are some examples.

\begin{tabular}{ccl} 
hours & distance & clockwise \\
\hline 2 and 7 & 5 & 2 to 7 \\
3 and 10 & 6 & 10 to 3 \\
2 and J & 4 & J to 2 \\
A and 8 & 6 & 8 to A
\end{tabular}

3. There are 6 arrangements of three objects.

The remaining three cards can be ordered as small, medium, and large. ${ }^{1}$ Now rearrange them properly to tell the magician what number he should add (clockwise) to the first card to get the number on the secret card. Let's agree on this:

\begin{tabular}{cc} 
arrangement & distance \\
\hline sml & 1 \\
sIm & 2 \\
msl & 3 \\
mls & 4 \\
Ism & 5 \\
Ims & 6
\end{tabular}

${ }^{1}$ First by numerical order; for cards with the same number, order by suits: 


\subsection{Examples}

If, for example, you, the assistant, want to tell the magician that he should add 4 to the number (clockwise) on the first card, deal the medium as the second card, the large as the third, and the small as the fourth card. Here are two examples.

1. Suppose you have $\mathbf{\$} 5$, $\mathbf{\$} 7, \diamond J$, $\mathbf{\$} 4$, and $\mathbf{\$ Q}$, and you decide to use the \& cards for the first and the secret ones. The distance between \$7 and \$4 is of course 3, clockwise from \$4 to \$7. You therefore show $\mathbf{9} 4$ as the first card, and arrange the other three cards, $5, \diamond \mathrm{J}$, and $\mathbf{\$} \mathbf{Q}$, in the order medium, small, large. The second card is $\diamond \mathrm{J}$, the third $\mathbf{\$} 5$, and the fourth $\mathbf{M Q}$.

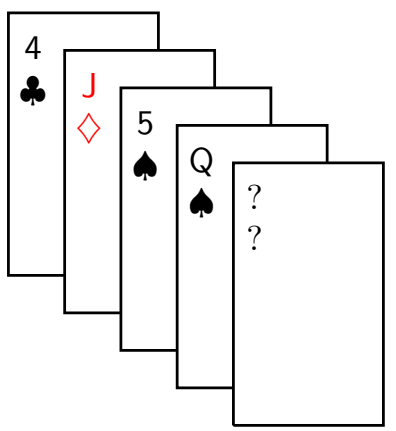

The secret card is $\mathbf{p} 7$.

2. Now to the magician. Suppose your assistant show you these four cards in order:

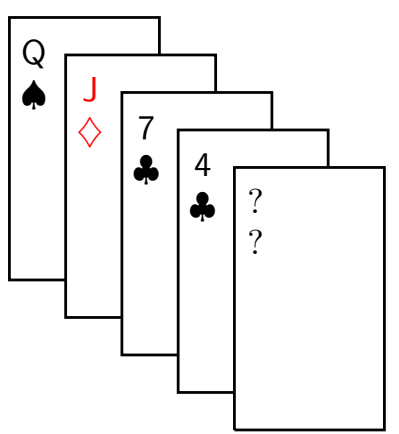

Then you know that the secret card is a $\mathbf{s}$, and you get the number by adding to $Q$ the number determined by the order large, medium, small, which is 6. Going clockwise, this is 5. The secret card is 5 . 


\section{Exercise}

1. For the assistant:
(a) \$5, \$7, $>6$, \$5, \$Q.
(b) $\nabla 2, \mathbf{A J}, \nabla K, \boldsymbol{\$} 2, \mathbf{4} 8$.

2. For the magician: what is the secret card?
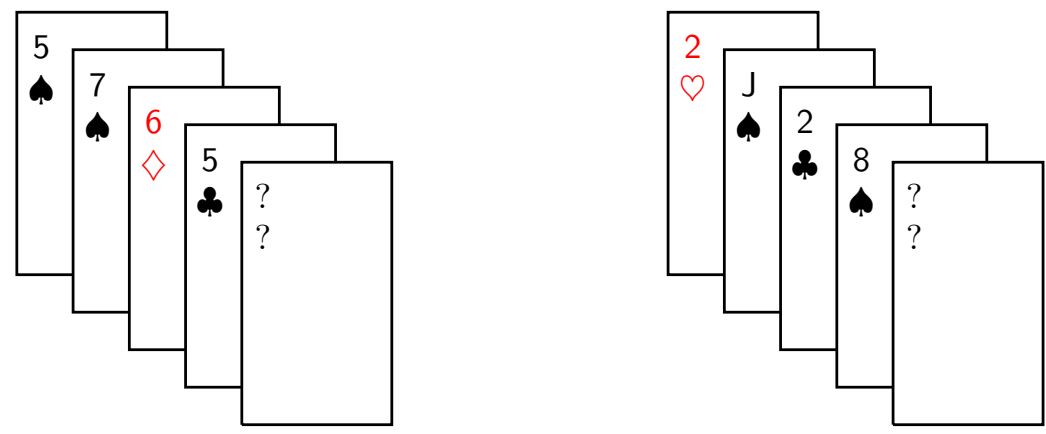


\section{Chapter 13}

\section{Digit problems}

1 When can you cancel illegitimately and yet get the correct answer?

2 A multiplication problem

3 A division problem

4 The most famous Monthly problem

5 The problem of $4 n$ 's

Appendix: Squares with 9 distinct nonzero digits

Appendix: Squares with 10 distinct digits

Exercise

Charles Twigg's square number puzzle

Cross number puzzle

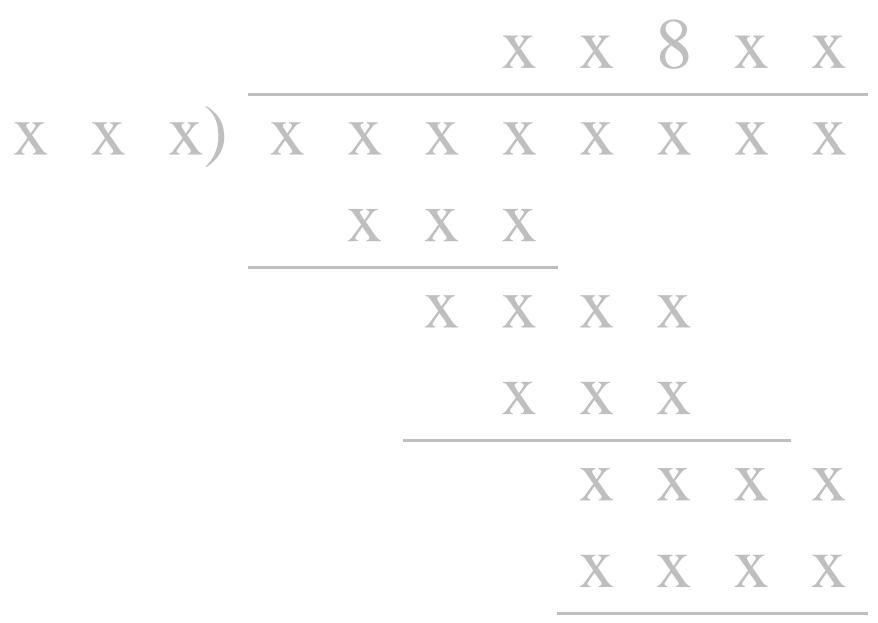




\subsection{When can you cancel illegitimately and yet get the correct answer?}

Let $a b$ and $b c$ be 2-digit numbers. When do such illegimate cancellations as

$$
\frac{a b}{b c}=\frac{a \not b}{\not b c}=\frac{a}{c}
$$

give correct results in lowest terms?

How about

$$
\frac{a \not b \not c}{\not b \not c d}=\frac{a}{d},
$$

allowing perhaps further simplifications of $\frac{a}{d}$ ? 


\subsection{A Multiplication problem}

A multiplication of a three-digit number by 2-digit number has the form

\begin{tabular}{ccccc} 
& & $p$ & $p$ & $p$ \\
$\times$ & & & $p$ & $p$ \\
\hline & $p$ & $p$ & $p$ & $p$ \\
$p$ & $p$ & $p$ & $p$ & \\
\hline$p$ & $p$ & $p$ & $p$ & $p$
\end{tabular}

in which all digits involved are prime numbers. Reconstruct the multiplication. 


\subsection{A division problem}

Reconstruct the division problem

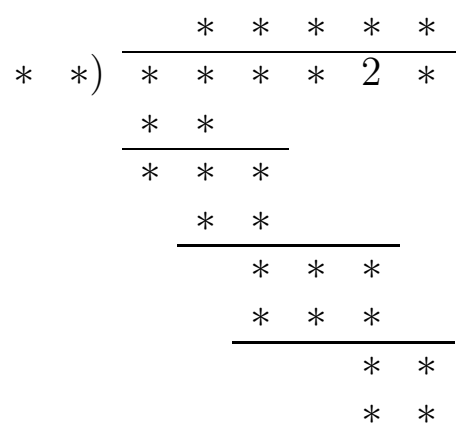

Charles Twigg: If the digit 2 is replaced by 9, the answer is also unique.

If the dividend is prefixed by another asterisk, then there are two solutions. 


\section{Another division problem: Problem E1 of the Monthly}

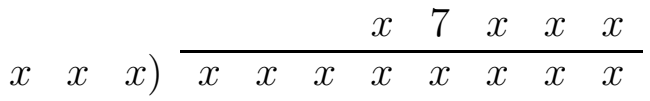

$$
\begin{aligned}
& \begin{array}{lllll}
x & x & x & x & \\
\hline & & x & x & x
\end{array} \\
& \begin{array}{llll}
x & x & x & \\
\hline x & x & x & x
\end{array} \\
& \begin{array}{lllll}
x & x & x & & \\
\hline & x & x & x & x
\end{array} \\
& \begin{array}{llll}
x & x & x & x
\end{array}
\end{aligned}
$$

Clearly, the last second digit of the quotient is 0 .

Let the divisor be the 3-digit number $d$.

Consider the 3-digit number in the seventh line, which is a multiple of $d$. Its difference from the 4-digit number in the sixth line is a 2-digit number. This must be $9 x x$.

This cannot be the same as the 3-digit number in the fifth line, since the difference between the 3-digit numbers in the fourth and fifth lines is a 3-digit number.

Therefore, in the quotient, the digit after 7 is a larger one, which must be smaller than the first and the last digits, since these give 4-digit multiples of $d$.

It follows that the quotient is 97809.

Since $8 d$ is a 3 -digit number $9 x x$, the 4 -digit number in the third and bottom lines is $9 d=10 x x$ or $11 x x$.

From this $8 d$ must be $99 x$, and therefore $992=8 \times 124$.

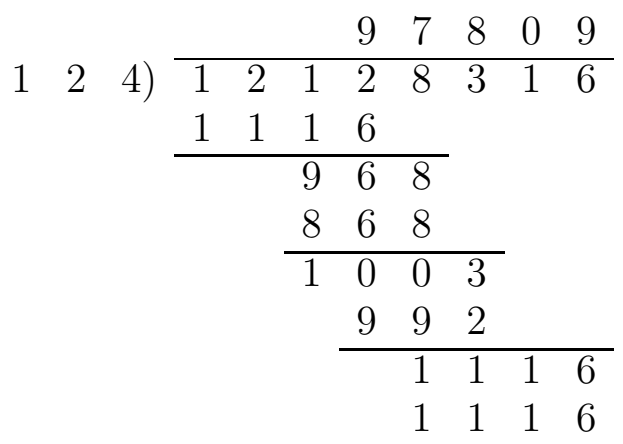




\section{One more division problem: not even a single known digit}

This is Problem E10 of the Monthly, by Fitch Cheney:

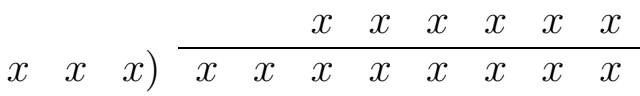

$$
\begin{aligned}
& \begin{array}{llll}
x & x & x & \\
\hline x & x & x & x
\end{array} \\
& \begin{array}{llllll}
x & x & x & & & \\
\hline & & x & x & x & x
\end{array} \\
& \begin{array}{llll}
x & x & x & \\
\hline x & x & x & x
\end{array} \\
& \begin{array}{llll}
x & x & x
\end{array}
\end{aligned}
$$




\subsection{The most popular Monthly problem}

The following problem, E 1111, is said to be the most popular problem in the American Mathematical Monthly. It was proposed by P. L. Chessin and appeared in the April issue of 1954.

Our good friend and eminent numerologist, Professor Euclide Paracelso Bombasto Umbugio, has been busily engaged testing on his desk calculator the $81 \cdot 10^{9}$ possible solutions to the problem of reconstructing the following exact long division in which the digits indiscriminately were each replaced by $x$ save in the quotient where they were almost entirely omitted.

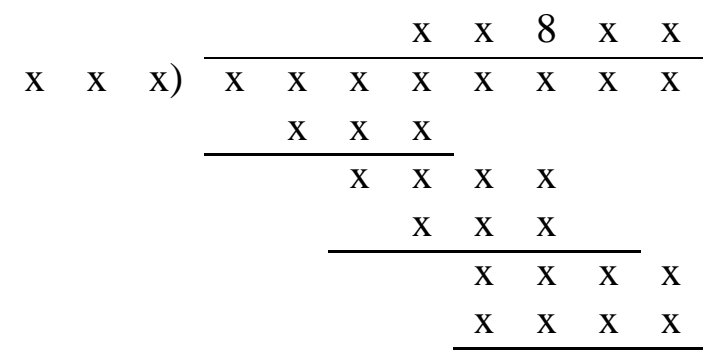

Deflate the Professor! That is, reduce the possibilities to $(18$. $\left.10^{9}\right)^{0}$.

Martin Gardner's remark: Because any number raised to the power of zero is one, the reader's task is to discover the unique reconstruction of the problem. The 8 is in correct position above the line, making it the third digit of a five-digit answer. The problem is easier than it looks, yielding readily to a few elementary insights. 


\subsection{The problem of $4 n$ 's}

\begin{tabular}{|c|c|}
\hline & $=\frac{n+n}{n+n}$ \\
\hline 2 & $=\frac{n}{n}+\frac{n}{n}$ \\
\hline 3 & $=\frac{n+n+n}{n}$ \\
\hline 4 & $=\frac{n-\cdot \bar{n}}{\bar{n}+\bar{n}}$ \\
\hline 5 & $=\frac{h+\cdot \bar{n}}{\bar{n}+\bar{n}}$ \\
\hline 6 & $=\left(\frac{n+n+n}{n}\right) !$ \\
\hline 7 & $=\frac{n-\cdot \bar{n}-\bar{n}}{\bar{n}}$ \\
\hline 8 & $=\frac{n}{\bar{n}}-\frac{n}{n}$ \\
\hline 9 & $=\frac{n}{\bar{n}} \cdot \frac{n}{n}$ \\
\hline 10 & $=\frac{n}{\bar{n}}+\frac{n}{n}$ \\
\hline 11 & $=\frac{n}{n}+\frac{n}{n}$ \\
\hline & $=\frac{n+. n+. n}{n}$ \\
\hline
\end{tabular}

\begin{tabular}{|l|}
\hline $13=\frac{n}{n}+\sqrt{\frac{n}{n}}$ \\
\hline $14=\frac{\sqrt{n(n+n)}}{n}$ \\
\hline $15=\frac{n}{\bar{n}}+\left(\sqrt{\frac{n}{n}}\right) !$ \\
\hline $16=\frac{n}{n}+\left(\sqrt{\frac{n}{n}}\right) !$ \\
\hline $17=\frac{n+n-\cdot \bar{n}}{n}$ \\
\hline $18=\frac{n}{\bar{n}}+\frac{n}{\bar{n}}$ \\
\hline $19=\frac{n}{n}+\frac{n}{\bar{n}}$ \\
\hline $20=\frac{n}{n}+\frac{n}{n}$ \\
\hline $21=\frac{n+n+\bar{n}}{\bar{n}}$ \\
\hline$\vdots$ \\
\hline
\end{tabular}

Theorem 13.1 (Hoggatt and Moser). Let $n$ be any positive number different from 1 and let $p$ be any integer greater than 3. Every integer may be expressed by using $p$ n's and a finite number of operator symbols used in high school texts.

Proof. It is easily verified that for every positive integer $k$,

$$
\begin{aligned}
\log _{\log _{\sqrt{n}} n} \log _{\sqrt{\sqrt{\cdots \sqrt{n}}}} n & =k, \\
\log _{\frac{n+n}{n}} \log _{\sqrt{\sqrt{\cdots \sqrt{n}}}} n & =k .
\end{aligned}
$$

where the base of the second logarithms contains $k$ square root signs. These settle the cases of 4 and 5 numbers. For higher values of $p$, add an appropriate numbers of $(n-n)+\cdots+(n-n)$. 


\section{Appendix: Squares with 9 distinct nonzero digits}

\begin{tabular}{|l|l||l|l||l|l||l|l|}
\hline 11826 & 139854276 & 12363 & 152843769 & 12543 & 157326849 & 14676 & 215384976 \\
\hline 15681 & 245893761 & 15963 & 254817369 & 18072 & 326597184 & 19023 & 361874529 \\
\hline 19377 & 375468129 & 19569 & 382945761 & 19629 & 385297641 & 20316 & 412739856 \\
\hline 22887 & 523814769 & 23019 & 529874361 & 23178 & 537219684 & 23439 & 549386721 \\
\hline 24237 & 587432169 & 24276 & 589324176 & 24441 & 597362481 & 24807 & 615387249 \\
\hline 25059 & 627953481 & 25572 & 653927184 & 25941 & 672935481 & 26409 & 697435281 \\
\hline 26733 & 714653289 & 27129 & 735982641 & 27273 & 743816529 & 29034 & 842973156 \\
\hline 29106 & 847159236 & 30384 & 923187456 & & & & \\
\hline
\end{tabular}

\section{Appendix: Squares with 10 distinct digits}

\begin{tabular}{|l|l||l|l||l|l||l|l|}
\hline 32043 & 1026753849 & 32286 & 1042385796 & 33144 & 1098524736 & 35172 & 1237069584 \\
\hline 35337 & 1248703569 & 35757 & 1278563049 & 35853 & 1285437609 & 37176 & 1382054976 \\
\hline 37905 & 1436789025 & 38772 & 1503267984 & 39147 & 1532487609 & 39336 & 1547320896 \\
\hline 40545 & 1643897025 & 42744 & 1827049536 & 43902 & 1927385604 & 44016 & 1937408256 \\
\hline 45567 & 2076351489 & 45624 & 2081549376 & 46587 & 2170348569 & 48852 & 2386517904 \\
\hline 49314 & 2431870596 & 49353 & 2435718609 & 50706 & 2571098436 & 53976 & 2913408576 \\
\hline 54918 & 3015986724 & 55446 & 3074258916 & 55524 & 3082914576 & 55581 & 3089247561 \\
\hline 55626 & 3094251876 & 56532 & 3195867024 & 57321 & 3285697041 & 58413 & 3412078569 \\
\hline 58455 & 3416987025 & 58554 & 3428570916 & 59403 & 3528716409 & 60984 & 3719048256 \\
\hline 61575 & 3791480625 & 61866 & 3827401956 & 62679 & 3928657041 & 62961 & 3964087521 \\
\hline 63051 & 3975428601 & 63129 & 3985270641 & 65634 & 4307821956 & 65637 & 4308215769 \\
\hline 66105 & 4369871025 & 66276 & 4392508176 & 67677 & 4580176329 & 68763 & 4728350169 \\
\hline 68781 & 4730825961 & 69513 & 4832057169 & 71433 & 5102673489 & 72621 & 5273809641 \\
\hline 75759 & 5739426081 & 76047 & 5783146209 & 76182 & 5803697124 & 77346 & 5982403716 \\
\hline 78072 & 6095237184 & 78453 & 6154873209 & 80361 & 6457890321 & 80445 & 6471398025 \\
\hline 81222 & 6597013284 & 81945 & 6714983025 & 83919 & 7042398561 & 84648 & 7165283904 \\
\hline 85353 & 7285134609 & 85743 & 7351862049 & 85803 & 7362154809 & 86073 & 7408561329 \\
\hline 87639 & 7680594321 & 88623 & 7854036129 & 89079 & 7935068241 & 89145 & 7946831025 \\
\hline 89355 & 7984316025 & 89523 & 8014367529 & 90144 & 8125940736 & 90153 & 8127563409 \\
\hline 90198 & 8135679204 & 91248 & 8326197504 & 91605 & 8391476025 & 92214 & 8503421796 \\
\hline 94695 & 8967143025 & 95154 & 9054283716 & 96702 & 9351276804 & 97779 & 9560732841 \\
\hline 98055 & 9614783025 & 98802 & 9761835204 & 99066 & 9814072356 & & \\
\hline
\end{tabular}




\section{Exercise}

1. Lucky Larry, a mathematics student whose plausible mistakes in computation always results in correct answers, once wrote an answer in the form

$$
a^{b} c^{a}=a b c a
$$

where $a b c a$ represents a four-digit integer whose digits $a, b, c$ are all different. What specific number did Lucky Larry write?

2. Find all natural numbers whose square (in base 10) is represented by odd digits only.

3. Let $N$ be the sum of the digits of a natural number $A$, let $B=$ $A+N$, let $A^{\prime}$ be the sum of the digits of the number $B$, and let $C=B+A^{\prime}$. Find $A$ if the digits of $C$ are those of $A$ in reverse order.

Solution. If $A$ has $k$ digits, then $C$ cannot exceed $A$ by more than $18 k$. On the other hand, if $C$ is the reverse of $A$, then $C$ exceeds $A$ by at least $9 \cdot 10^{\frac{k-2}{2}}$ is $k$ is even, and by at least $99 \cdot 10^{\frac{k-3}{2}}$ if $k$ is odd. This quickly implies $k \leq 2$. From this, we find $A=12$ or 69 .

4. Find the three 3-digit numbers each of which is equal to the product of the sum of its digits by the sum of the squares of its digits.

5. Find all 4-digit numbers $a b c d$ such that $\sqrt[3]{a b c d}=a+b+c+d$.

6. Use each digit $1,2,3,4,5,6,7,8,9$ exactly once to form prime numbers whose sum is smallest possible.

What if we also include the digit 0 ?

7. There are exactly four 3-digit numbers each equal to the sum of the cubes of its own digits. Three of them are 153, 371, and 407. What is the remaining one?

8. Find digits $m, a, b, c, d, e, f$ such that

$$
\frac{a b c d e f}{f e d c b a}=\frac{9 m}{9 m+1} .
$$

9. Find a number of the form aaabbbccc, which when increased by 1 , gives a square. 
10. Here are seven consecutive squares for each of which its decimal digits sum to a square:

$$
\text { 81, 100, 121, 144, 169, 196, } 225 .
$$

Find another set of seven consecutive squares with the same property. ${ }^{1}$

11. Find a perfect square of 12 digits formed from the juxtaposition of two squares, one having 4 digits and the other 8 digits.

12. A pandigital number is one whose decimal representation contains all digits $0,1, \ldots, 9$. There are three pandigital perfect squares whose square roots are palindromes. Find them.

13. Find the smallest 3-digit number $N$ such that the three numbers obtained by cyclic permutations of its digits are in arithmetic progression.

14. Form a square of 8 digits which is transformed into a second square when the second digit from the left is increased by 1 .

15. The number $(a b b b b)^{2}-1$ has 10 digits, all different. Find the number. ${ }^{2}$

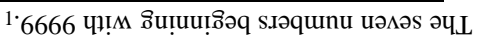

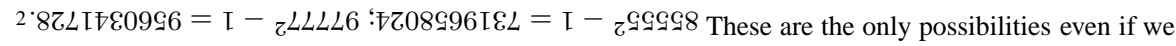
consider more generally numbers consisting of two consecutive blocks of repeating digits, whose squares, to within \pm 1 , contain all ten digits without repetition.
} 


\section{Charles Twigg's square number trivia}

What three-digit squares have the following characteristics?

1. are palindromes.

2. are permutations of consecutive digits.

3. form reversal pairs.

4. are three permutations of the same digit set.

5. three of its permutations are prime.

6. the sum of the digits is 19 .

7. is also a cube.

8. the central digit is perfect.

9. are composed of even digits.

10. the central digit is a nonzero cube. 


\section{Cross number puzzle}

Place 13 three - digit square numbers in the spaces in the accompanying grid. (The solution is unique).

\begin{tabular}{|l|l|l|l|l|l|l|l|l|l|l|}
\hline & & & & & & & & & & \\
\hline & & & & & & & & & & \\
\hline & & & & & & & & & & \\
\hline
\end{tabular}




\section{Project}

Arrange the ten digits $(0,1, \ldots, 9$, no repetition $)$ in a row

$$
\text { abcdefghij }
$$

so that the following 3-digit numbers in the table below are divisible by the prime below them.

$$
\begin{array}{ccccccc}
\text { bcd } & \text { cde } & \text { def } & \text { efg } & \text { fgh } & \text { ghi } & \text { hij } \\
\hline 2 & 3 & 5 & 7 & 11 & 13 & 17
\end{array}
$$

Solution: 1460357289 or 4160357289

Since de $f$ is divisible by $5, f$ must be 0 or 5 . Now, the number $f g h$ must be divisible by 11 . The only number that can be formed from the first two digits of $13 \mathrm{~m}$ by appending a 0 or a 5 on the left to form a multiple of 11 are $286,390,728$, and 832 . Clearly, $f=5$, and $f g h i$ is only one of

$$
\text { 5286, 5390, 5728, } 5832 .
$$

Now we want to find a multiple of 17 beginning with the last two digits of these. This eliminates the last case.

$$
52867,53901,57289 .
$$

Since $e f g$ is a multiple of $7, \bmod (e, 7)$ must satisfy $2 \bmod (e, 7)+$ $\bmod (10 f+g, 7) \equiv 0 \bmod 7$. Necessarily, $e=2,1,3$. Now we must have efghij $=357289$.

Now it remains to arrange $0,1,4,6$ as $a, b, c, d$ such that the three digit numbers $b c d, c d 3$ are 3 digit numbers divisible by 2, 3 respectively. In particular, $c+d+3$ is divisible by 3 . The only choice is $c=6$ and $d=0$. We have $a b 60357289 . a$ and $b$ can be 1,4 or 4,1 . 


\section{Chapter 14}

\section{Numbers with many repeating digits}

1 A quick multiplication

2 The repunits

3 Squares of repdigits

4 Sorted numbers with sorted squares

Appendix: Factorization of $10^{n}+1$ for $n \leq 50$

Exercise 


\subsection{A quick multiplication}

What is the smallest positive integer with the property that when the digit 1 is appended to both ends, the new number is 99 times the original?

Suppose the number $X$ has $n$ digits. We require $10^{n}+10 X+1=99 X$ or $89 X=10^{n}+1$. Note that 89 is prime. To find the smallest $n$ we divide 89 into the number 1 followed by a string of zeros, extended if necessary, until a remainder 80 occurs. Then add 1 and obtain an integer quotient which is the smallest possible $X$.

The first time this occurs is at the 22 nd zero. Thus, the smallest possible value of $X$ is

$$
\frac{10^{22}+1}{89}=112359550561797752809 .
$$

For this value of $X$ we have

$$
99 \times 112359550561797752809=11123595505617977528091 .
$$

See Appendix for the factorization of numbers of the form $10_{n-1} 1$. 


\subsection{The repunits}

The repunit $R_{n}$ is the number whose decimal representation consists of a string of $n$ ones. Thus, $R_{n}=1_{n}$.

Notations

$$
\begin{aligned}
\mathbf{A} & :=123456790, \\
\mathbf{B} & :=098765432, \\
\overline{\mathbf{1}}_{k} & :=1_{k-1}, \\
\overline{\mathbf{2}}_{k} & :=12_{k}, \\
\overline{\mathbf{m}}_{k} & :=12 \cdot(m-1) m_{k}(m-1) \cdots 2, \quad \text { for } 3 \leq m \leq 9 .
\end{aligned}
$$

Theorem 14.1. Let $p=9 k+m, k \geq 0,1 \leq m \leq 9$. For $q \geq p$,

$$
R_{p} R_{q}=\mathbf{A}_{k} \overline{\mathbf{m}}_{q-p+1} \mathbf{B}_{k} 1 .
$$

In particular,

$$
\left(R_{p}\right)^{2}=\mathbf{A}_{k} \overline{\mathbf{m}}_{k} \mathbf{B}_{k} 1
$$




\subsection{Squares of repdigits}

In the decimal representations of integers, we write $a_{n}$ for a string of $n$ digits each equal to $a$.

Theorem 14.2. For $n \geq 2$,

$$
\begin{aligned}
& \left(3_{n}\right)^{2}=1_{n-1} 08_{n-1} 9, \\
& \left(6_{n}\right)^{2}=4_{n-1} 35_{n-1} 6, \\
& \left(9_{n}\right)^{2}=9_{n-1} 89_{n-1} 1 .
\end{aligned}
$$

Proof. The last one is easiest.

$$
\begin{aligned}
\left(9_{n}\right)^{2} & =\left(10^{n}-1\right)^{2} \\
& =10^{2 n}-2 \cdot 10^{n}+1 \\
& =10^{n}\left(10^{n}-2\right)+1 \\
& =9_{n-1} 80_{n-1} 1 .
\end{aligned}
$$

From this we obtain the square of $3_{n}$ by division by 9 , then the square of $6_{n}$ by multiplication by 4 .

Theorem 14.3. Let $n=9 k+m, k \geq 0,1 \leq m \leq 9$. For $a=$ $1,2,4,5,7,8$,

$$
\left(a R_{n}\right)^{2}=\mathbf{A}_{k} \overline{\mathbf{m}} \mathbf{B}_{k} c,
$$

where $\mathbf{A}, \mathbf{B}$ and $c$ are given by

\begin{tabular}{|r||r|r|r|}
\hline$a$ & $\mathbf{A}$ & $\mathbf{B}$ & $c$ \\
\hline \hline 1 & 123456790 & 098765432 & 1 \\
\hline 2 & 493827160 & 395061728 & 4 \\
\hline 4 & 197530864 & 580246913 & 5 \\
\hline 5 & 308641975 & 469135802 & 6 \\
\hline 7 & 604938271 & 839506172 & 9 \\
\hline 8 & 790123456 & 320987654 & 4 \\
\hline
\end{tabular}

and $\mathbf{m}$ is given by

\begin{tabular}{|c||c|r|r|r|r|r|r|r|r|}
\hline$a$ & 1 & 2 & 3 & 4 & 5 & 6 & 7 & 8 & 8 \\
\hline \hline 1 & & 12 & 1232 & 123432 & 12345432 & 1234565432 & 123456765432 & 12345678765432 & 1234567898765432 \\
\hline 2 & & 48 & 4928 & 493728 & 49381728 & 4938261728 & 493827061728 & 49382715061728 & 4938271595061728 \\
\hline 4 & 1 & 193 & 19713 & 1974913 & 197526913 & 19753046913 & 1975308246913 & 197530860246913 & 19753086380246913 \\
\hline 5 & 2 & 302 & 30802 & 3085802 & 308635802 & 30864135802 & 3086419135802 & 308641969135802 & 30864197469135802 \\
\hline 7 & 4 & 592 & 60372 & 6048172 & 604926172 & 60493706172 & 6049381506172 & 604938259506172 & 60493827039506172 \\
\hline 8 & 6 & 774 & 78854 & 7899654 & 790107654 & 79012187654 & 7901232987654 & 790123440987654 & 79012345520987654 \\
\hline
\end{tabular}




\subsection{Sorted numbers with sorted squares}

A number is sorted if its digits are nondecreasing from left to right. It is strongly sorted if its square is also sorted. It is known that the only strongly sorted integers are given in the table below. ${ }^{1}$

- $1,2,3,6,12,13,15,16,38,116,117$.

- $16_{n} 7$.

- $3_{n} 4$.

- $3_{n} 5$.

- $3_{m} 6_{n} 7$.

$$
\begin{aligned}
\left(3_{n} 5_{1}\right)^{2} & =\left(10 \cdot 3_{n}+5\right)^{2} \\
& =100 \cdot\left(3_{n}\right)^{2}+100 \cdot\left(3_{n}\right)+25 \\
& =1_{n-1} 08_{n-1} 9_{1} 0_{2}+3_{n} 25 \\
& =1_{n-1} 12_{n-1} 225 \\
& =1_{n} 2_{n+1} 5 .
\end{aligned}
$$

If $x=3_{m} 6_{n} 7$, then $3 x=10_{m-1} 10_{n} 1$, and it is easy to find its square.

$$
\left(3_{m} 6_{n} 7\right)^{2}= \begin{cases}1_{m} 3_{m} 4_{n-m+1} 6_{m} 8_{n} 9, & \text { if } n+1 \geq m, \\ 1_{m} 3_{n+1} 5_{m-n-1} 6_{n+1} 8_{n} 9, & \text { if } n+1<m .\end{cases}
$$

More generally, the product of any two numbers of the form $3_{m} 6_{n} 7$ is sorted.

\footnotetext{
${ }^{1}$ Problem 1234, Math. Mag., 59 (1986) 1, solution, 60 (1987)1. See also R. Blecksmith and C. Nicol, Monotonic numbers, Math. Mag., 66 (1993) 257-262.
} 


\section{Appendix: Factorization of $10^{n}+1$ for $1 \leq n \leq 50$}

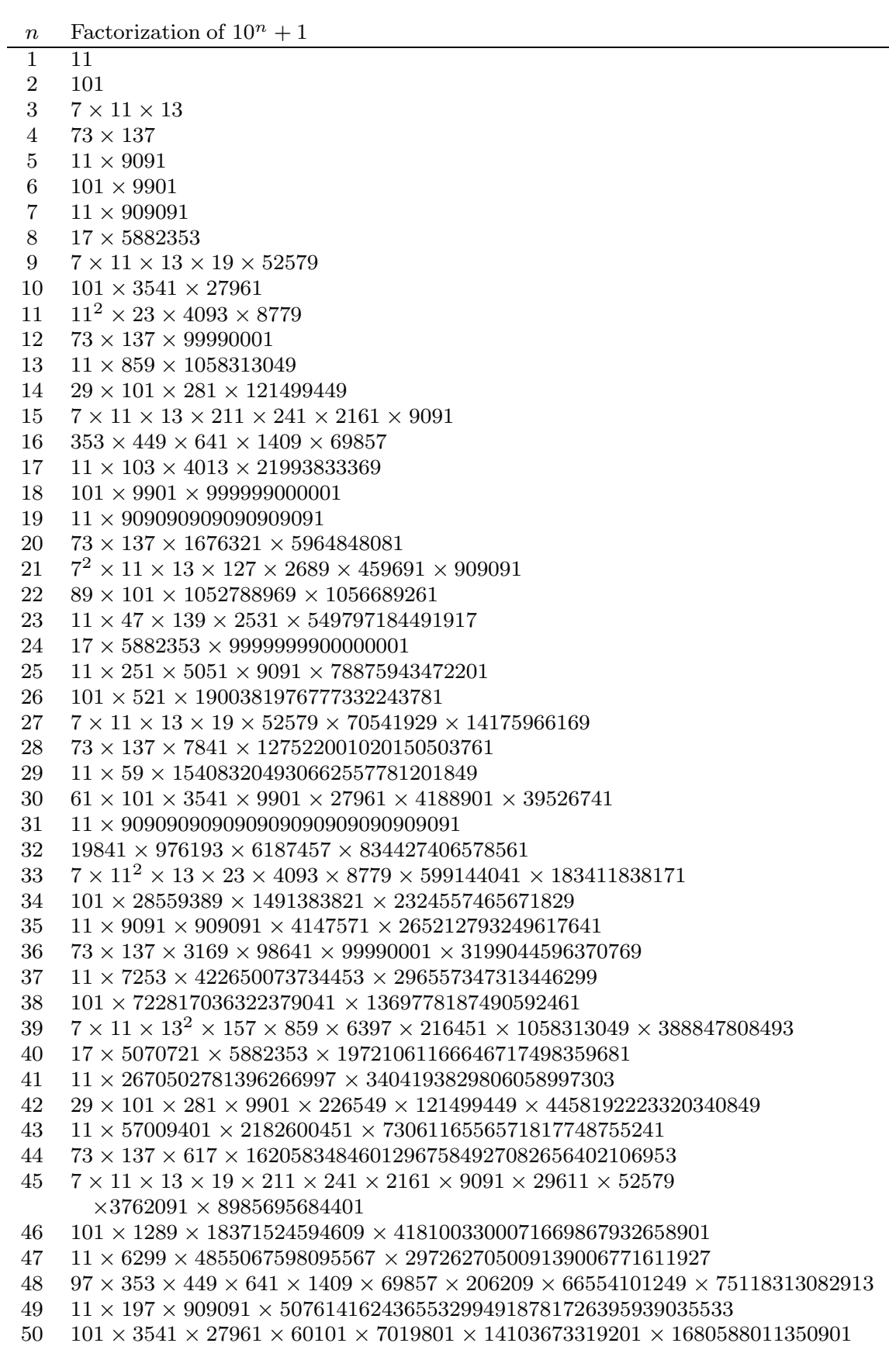




\section{Exercise}

1. Show that

$$
\frac{16_{n}}{6_{n} 4}=\frac{1}{4}, \quad \frac{19_{n}}{9_{n} 5}=\frac{1}{5}, \quad \frac{26_{n}}{6_{n} 5}=\frac{2}{5}, \quad \frac{49_{n}}{9_{n} 8}=\frac{4}{8} .
$$

2. Show that $\left(16_{n} 7\right)^{2}=27_{n} 8_{n+1} 9$.

3. $\left(3_{n} 4\right)^{2}=1_{n+1} 5_{n} 6$.

4. John shook its head. "Multiply that huge number by 8 in my head? You've got to be kidding."

"But it's easy, Dad." Mike told him. "You just shift its last digit to the front."

The boy was right, and it is the smallest number for which it works. What was the number?

5. John looked over his son's shoulder. "That must be an interesting number," he said. "Homework?"

"Just fun, Dad," Doug replied. "It's the serial number on that clock you brought back from Kaloat, and I've just noticed something special about it. If you take the last two digits and put them in front, you get exactly four times the original number, and it's the smallest number that works that way."

What was the serial number?

6. Given an integer $n$. Show that an integer can always be found which contains only digits 0 and 1 (in the decimal scale) and which is divisible by $n .^{2}$

7. Determine an $n$-digit number such that the number formed by reversing the digits is nine times the original number. What other numbers besides nine are possible?

8. Write $5_{2} 9_{n-2} 893_{n-1} 9$ as a sum of three squares of natural numbers.

9. There are only two repdigits $a_{n}$ whose squares have digital sum 37 . What are these? ${ }^{3}$

\footnotetext{
${ }^{2}$ AMM Problem 4281.

${ }^{3}$ Answer: ${ }^{-\top} L$ pue ${ }^{\dagger} Z$
} 


\title{
Chapter 15
}

\section{Digital sum and digital root}

\author{
1 Digital sum sequences \\ 2 Digital root \\ 3 Digital roots of the powers of 2 \\ 4 Digital root sequences \\ Exercise
}




\subsection{Digital sum sequences}

The digital sum of a positive integer $n$ is the sum of the digits of $n$. We denote this by $d(n)$.

Given a positive integer $a$, the digit sum sequence $\mathcal{S}(a)=\left(a_{n}\right)$ is defined recursively by

$$
a_{n+1}=a_{n}+d\left(a_{n}\right), \quad a_{1}=a .
$$

Here are the first few digit sum sequences:

$\mathcal{S}(1) \quad 1,2,4,8,16,23,28,38,49,62,70,77,91,101,103,107, \ldots$

$\mathcal{S}(3) \quad 3,6,12,15,21,24,30,33,39,51,57,69,84,96,111,114, \ldots$

$\mathcal{S}(5) \quad 5,10,11,13,17,25,32,37,47,58,71,79,95,109,119,130, \ldots$

$\mathcal{S}(7) \quad 7,14,19,29,40,44,52,59,73,83,94,107,115,122,127,137, \ldots$

$\mathcal{S}(9) \quad 9,18,27,36,45,54,63,72,81,90,99,117,126,135,144,153, \ldots$

Note that they are quite similar to the digital root sequences.

Show that $\mathcal{S}(3)=\mathcal{R}(3)$ and $\mathcal{R}(9)=\mathcal{S}(9)$.

What is the smallest number that does not appear in any of these digit sum sequences?

Find the first 10 terms of the digital sum sequence beginning with this number.

$20,22,26,34,41,46,56,67,80,88,104,109,119,130,134,142, \ldots$

Find the next smallest number which is not in any of the 6 digit sum sequences and generate a new digit sum sequence from it.

$31,35,43,50,55,65,76,89,106,113,118,128,139,152,160,167, \ldots$

There are infinitely many digit sum sequences because there are infinitely many numbers which are not of the form $n+d(n)$.

The number 101 is $n+d(n)$ for $n=91$ and 100 .

The number 101 traces back to 100,86 which is a starter. It also traces back to 91, and eventually 1 .

Here are the numbers below 100 which are not of this form:

$$
1,3,5,7,9,20,31,42,53,64,75,86,97 .
$$

An infinite sequence of "starters": $10_{n} 122, n \geq 1$. Every number $n \leq 10_{n} 114$ has $n+d(n) \leq 10_{n} 121$; every number $n \geq 10_{n} 115$ has $d(n) \geq 10_{n} 123$. 


\subsection{Digital root}

Given a positive integer $n$, let $\mathbf{d}(n)$ be the sum of the digits of $n$. If the operation $\mathbf{d}$ is repeated indefinitely, it stabilizes after a finite number of steps and yield a number between 1 and 9, which we call the digital root of $n$, denoted $\mathbf{D}(n)$. See [Dudeny, Amusements, p.157].

Theorem 15.1. 1. $\mathbf{D}(m+n)=\mathbf{D}(\mathbf{D}(m)+\mathbf{D}(n))$.

2. $\mathbf{D}(m n)=\mathbf{D}(\mathbf{D}(m) \mathbf{D}(n))$.

3. $\mathbf{D}\left(m^{n}\right)=\mathbf{D}\left(\mathbf{D}(m)^{n}\right)$.

4. $\mathbf{D}(\mathbf{D}(n))=\mathbf{D}(n)$.

5. $\mathbf{D}(n+9)=\mathbf{D}(n)$.

6. $\mathbf{D}(9 n)=9$.

Proof. (5) $\mathbf{D}(n+9)=\mathbf{D}(\mathbf{D}(n)+\mathbf{D}(9))=\mathbf{D}(\mathbf{D}(n)+9)=\mathbf{D}(n)$ since $\mathrm{D}(n)$ is a single-digit number.

(6) $\mathbf{D}(9 n)=\mathbf{D}(9 \mathbf{D}(n))=9$ since $\mathbf{D}(n)$ has one single digit. 


\subsection{The digital roots of the powers of 2}

$\begin{array}{cccccccc}n & 0 & 1 & 2 & 3 & 4 & 5 & 6 \\ 2^{n} & 1 & 2 & 4 & 8 & 16 & 32 & 64 \\ \mathbf{D}\left(2^{n}\right) & 1 & 2 & 4 & 8 & 7 & 5 & 1\end{array}$

It follows that the sequence of digital roots of $2^{n}$ is periodic with period 6.

\begin{tabular}{|l|l|}
\hline & sequence of digital roots \\
\hline \hline $2^{n}$ & $\overline{1,2,4,8,7,5}$ \\
$3^{n}$ & $1,3, \overline{9}$ \\
$4^{n}$ & $\frac{1,4,7}{1,5,7,8,4,2}$ \\
$5^{n}$ & $1,5, \overline{9}$ \\
$6^{n}$ & $1,6, \overline{9}$ \\
$7^{n}$ & $\frac{1,7,4}{1,8}$ \\
$8^{n}$ & $1, \overline{9}$ \\
$9^{n}$ & $1, \overline{9}$ \\
\hline
\end{tabular}

The sequence of the digital roots of $2^{n}+1$ is also periodic with period 6:

$$
2,3,5,9,8,6, \ldots
$$

\section{Digital roots of Fermat numbers}

To find the digital root of the Fermat number $F_{k}=2^{2^{k}}+1$, we need only find $2^{k} \bmod 6$. Now, it is clear that $2^{k} \equiv 1$ or $2 \bmod 3$ according as $k$ is even or odd. Therefore, $2^{k} \equiv 4$ or $\bmod 6$ according as $k$ is even or odd. From this, we have

$$
\mathbf{D}\left(F_{k}\right)= \begin{cases}8 & \text { if } k \equiv 0 \bmod 2 \\ 5 & \text { if } k \equiv 1 \bmod 2\end{cases}
$$

The digital roots of the Fibonacci numbers form a sequence of period 24:

$1,1,2,3,5,8,4,3,7,1,8,9,8,8,7,6,4,1,5,6,2,8,1,9,1,1, \ldots$ 


\subsection{Digital root sequences}

A digit root sequence $\left(a_{n}\right)$ is defined recursively by

$$
a_{n+1}=a_{n}+D\left(a_{n}\right), \quad a_{1}=a .
$$

The digital root sequence beginning with 1 is

$$
1,2,4,8,16,23,28,29,31,35,43,50,55,56,58,62,70, \ldots
$$

Obviously, the digital root sequence beginning with any of these terms is a subsequence of this. By taking a term not in this sequence we generate a new one. Here are five digital root sequences with initial terms 1, 3, 5, 7,9 .

$\mathcal{R}(1) \quad 1,2,4,8,16,23,28,29,31,35,43,50,55,56,58,62,70,77,82,83,85,89,97,104, \ldots$

$\mathcal{R}(3) \quad 3,6,12,15,21,24,30,33,39,42,48,51,57,60,66,69,75,78,84,87,93,96,102, \ldots$

$\mathcal{R}(5) \quad 5,10,11,13,17,25,32,37,38,40,44,52,59,64,65,67,71,79,86,91,92,94,98,106, \ldots$

$\mathcal{R}(7) \quad 7,14,19,20,22,26,34,41,46,47,49,53,61,68,73,74,76,80,88,95,100, \ldots$

$\mathcal{R}(9) \quad 9,18,27,36,45,54,63,72,81,90,99,108,117,126,135,144,153, \ldots$

Theorem 15.2 (Kumar). 1. The digital roots of these sequences are periodic.

2. These five sequences partition the natural numbers.

The digital roots of these sequences are

$$
\begin{array}{ll}
R_{1} & \overline{1,2,4,8,7,5} \\
R_{3} & \overline{3,6} \\
R_{5} & \overline{5,1,2,4,8,7} \\
R_{7} & \overline{7,5,1,2,4,8} \\
R_{9} & \overline{9}
\end{array}
$$




\section{Exercise}

1. A Filzian number is one which is the product of its digits and digial sum. For example, $144=1 \cdot 4 \cdot 4(1+4+4)$. Find one more such number. ${ }^{1}$

2. Find the digital root of $n$ !.

3. Find the digital root sequence of $n^{2}$.

4. The cubes.

5. The triangular numbers.

6. Find all integers $n$ such that the sum of the digits of $n^{2}$ is equal to $n$, i.e., $d\left(n^{2}\right)=n$.

7. What is the digital root of a number of the form $2^{n-1}\left(2^{n}-1\right)$ ?

\footnotetext{
${ }^{1}$ Answer: 135 . It is known that there are only finitely many Filzian numbers. Apart from 1 and 144, the other known one is 135 .
} 


\section{Project: Sum of squares of digits}

Let $f(n)$ be sum of the digits of $n$. For example, $f(1)=1, f(2)=4$, $f(10)=1, f(12)=5, f(123)=14$ etc.

Study the iterations of $f$.

For example, the iterations of $f$ beginning with 4 leads to the cycle

$$
4 \rightarrow 16 \rightarrow 37 \rightarrow 58 \rightarrow 89 \rightarrow 145 \rightarrow 42 \rightarrow 20 \rightarrow 4
$$




\section{Chapter 16}

\section{3-4-5 triangles in the square}
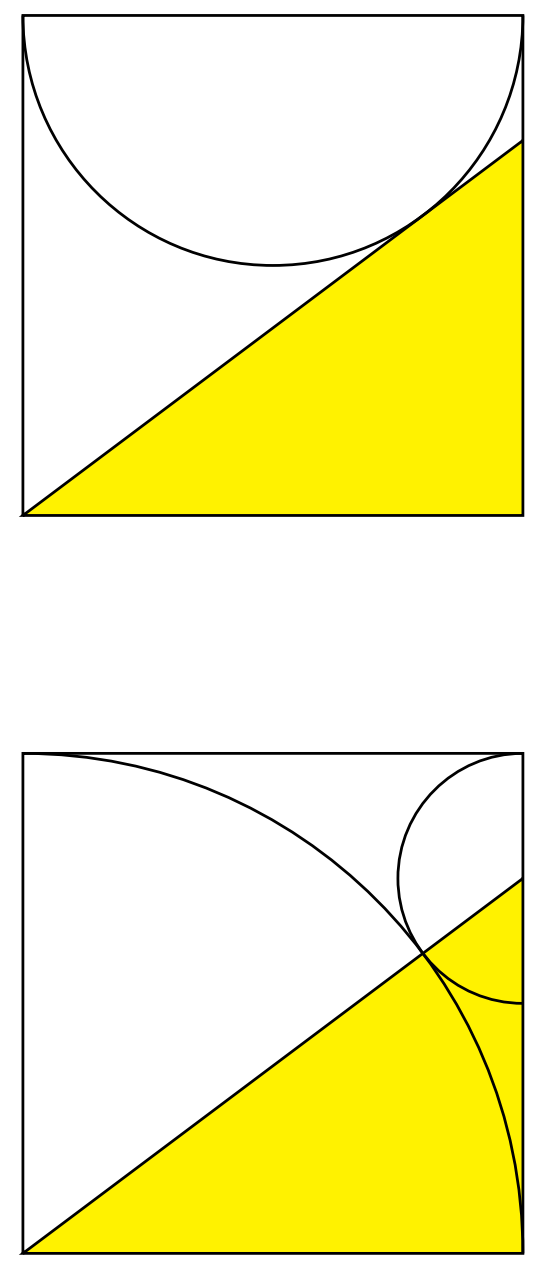

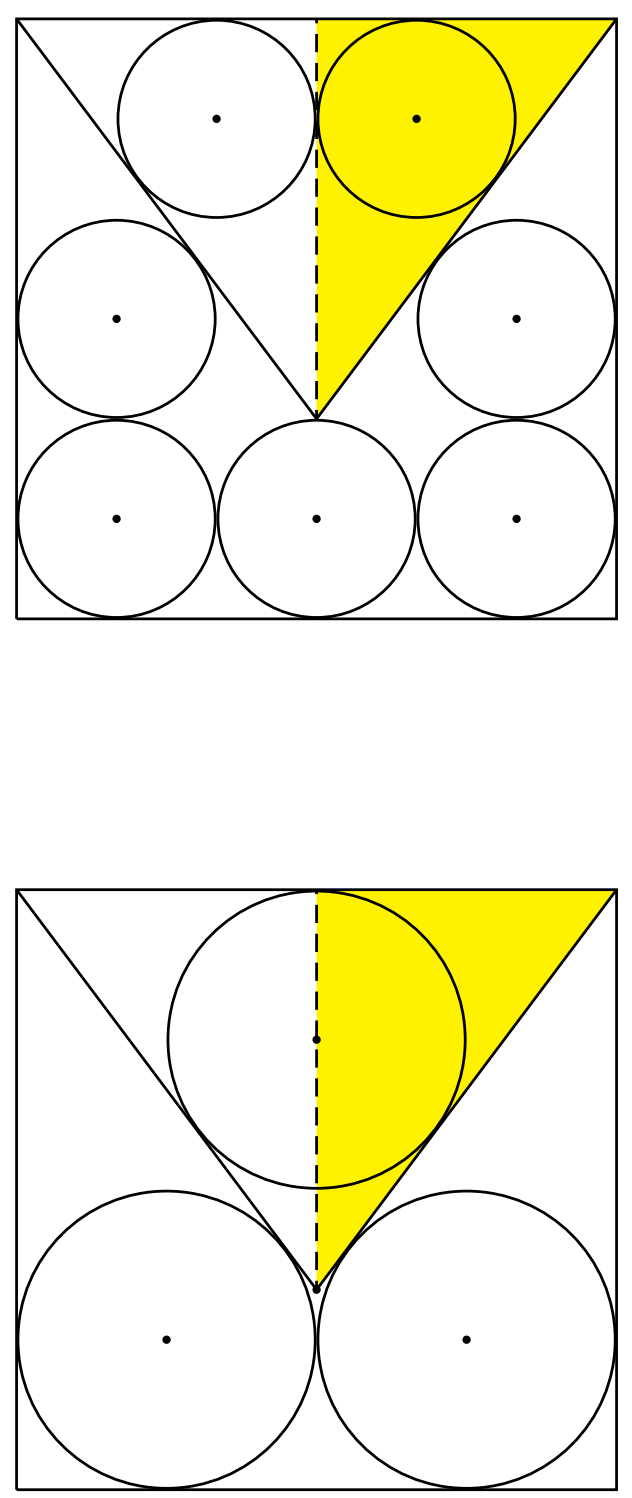


$$
98
$$




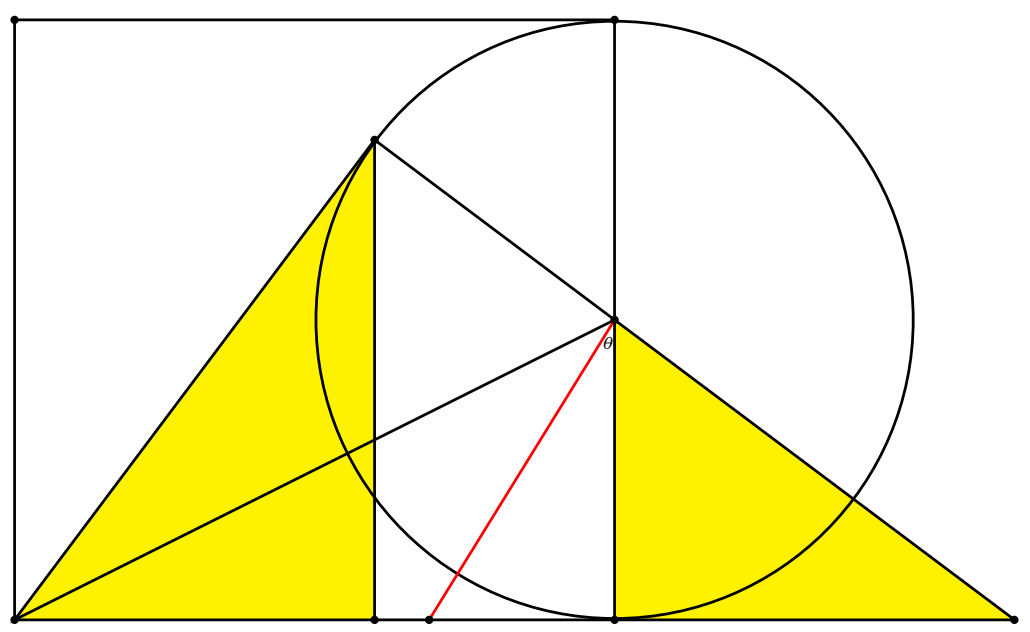

$\tan \theta=\frac{1}{\varphi}$. 


\section{Exercise}

1. Show that the shaded triangle is a $3: 4: 5$ triangle.

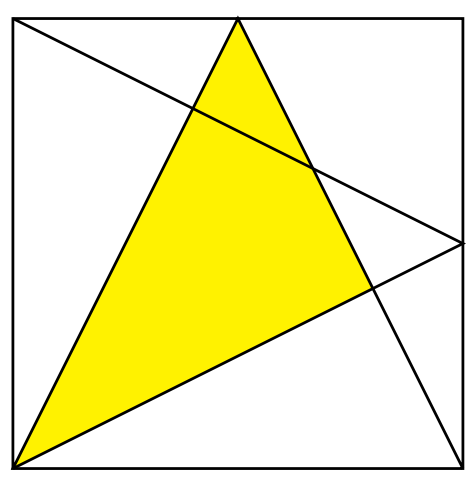

2. Find the ratio of the areas of $A B Y, B C X Y$, and $A X D$.

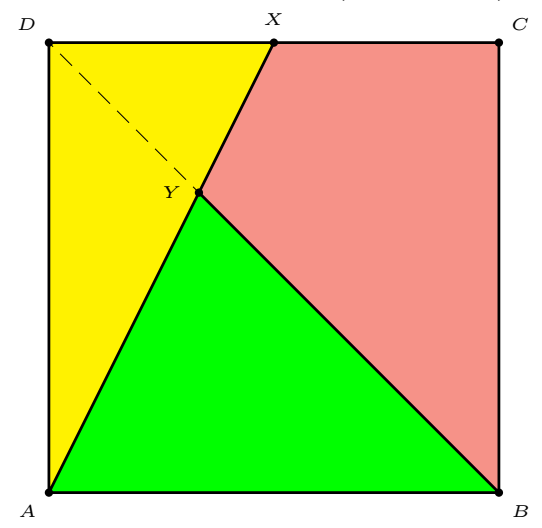




\section{Chapter 17}

\section{Combinatorial games}

1 Subtraction games

2 The nim sum

2 Nim

3 Northcott's variation of nim

4 Wythoff's game

Appendix: Beatty's theorem

Exercise

Project: Another subtraction game 


\subsection{Subtraction games}

Starting with a given positive integer $N$, two players alternately subtract a positive amount less than a given positive number $d<N$. The one who gets down to 0 wins.

Theorem 17.1. The player who secures a multiple of $d$ has a winning strategy.

\section{An equivalent version: the battle of numbers}

Starting with 0 , two players alternately add positive integers less than a given limit $d$. The one who gets to a specified $N$ wins.

The winning positions are the terms of the arithmetic progression of common difference $d$ containing $N$. Specifically, the small number mod $(N, d)$ is a winning position. Therefore, the first player has a winning strategy if and only if $N$ is not divisible by $d$. 


\subsubsection{The Sprague-Grundy sequence}

Let $\mathcal{G}$ be a 2-person counter game in which two players alternately remove a positive amount of counters according to certain specified rules. The Sprague-Grundy sequence of $\mathcal{G}$ is the sequence $(g(n))$ of nonnegative integers defined recursively as follows.

(1) $g(n)=0$ for all $n$ which have no legal move to another number. In particular, $g(0)=0$.

(2) Suppose from position $n$ it is possible to move to any of positions $m_{1}, m_{2}, \ldots, m_{k},($ all $<n)$, then $g(n)$ is the smallest nonnegative integer different from $g\left(m_{1}\right), g\left(m_{2}\right), \ldots, g\left(m_{k}\right)$.

Theorem 17.2. The player who secures a position $n$ with $g(n)=0$ has a winning strategy.

\section{Example 1: the trivial counter game}

If $\mathcal{G}$ is the game which the players may subtract any positive amount, the Sprague-Grundy sequence is the natural sequence

$$
0,1,2,3, \ldots, n, \ldots
$$

\section{Example 2: removing not more than $d$ counters}

If $\mathcal{G}$ is the game which subtracts numbers $<d$, then the Sprague-Grundy sequence is periodic $\overline{0,1,2, \ldots, d-1}$. The values of $n$ for which $g(n)=$ 0 are precisely the multiples of $d$. 


\subsubsection{Subtraction of square numbers}

Two players alternately subtract a positive square number. We calculate the Sprague-Grundy sequence.

$$
\begin{aligned}
& g(0)=0 \\
& 1 \longrightarrow 0 \Longrightarrow g(1)=1 \\
& g(2)=0 \\
& 3 \longrightarrow 2 \Longrightarrow g(3)=1 \\
& 4 \rightarrow 3,0 \Longrightarrow g(4)=2 \\
& 5 \rightarrow 4,1 \Longrightarrow g(5)=0 \\
& 6 \rightarrow 5,2 \Longrightarrow g(6)=1 \\
& 7 \rightarrow 6,3 \Longrightarrow g(7)=0 \\
& 8 \rightarrow 7,4 \Longrightarrow g(8)=1 \\
& 9 \rightarrow 8,5,0 \Longrightarrow g(9)=2 \\
& 10 \rightarrow 9,6,1 \Longrightarrow g(10)=0
\end{aligned}
$$

The values of $n \leq 1000$ for which $g(n)=0$ are as follows: ${ }^{1}$

Suppose we start with 74. Player A can subtract 64 to get 10 , which has value 0 . This means no matter how B moves, A can always win. This is clear if B moves to 9 or 1 . But if B moves to 6, then A can move to 5 which again has value 0 , since now B can only move to 4 or 1 .

\section{Exercise}

How would you win if the starting number is 200 ? or $500 ?$

\footnotetext{
${ }^{1}[$ Smith, p.68] incorrectly asserts that this sequence is periodic, with period 5.
} 


\subsubsection{Subtraction of square numbers}

The first 100 terms of the Sprague-Grundy sequence are

\begin{tabular}{cccccccccc}
1 & 2 & 3 & 4 & 5 & 6 & 7 & 8 & 9 & 10 \\
\hline 1 & 0 & 1 & 2 & 0 & 1 & 0 & 1 & 2 & 0 \\
1 & 0 & 1 & 2 & 0 & 1 & 0 & 1 & 2 & 0 \\
1 & 0 & 1 & 2 & 3 & 2 & 3 & 4 & 5 & 3 \\
2 & 3 & 4 & 0 & 1 & 2 & 3 & 2 & 0 & 1 \\
2 & 3 & 2 & 0 & 1 & 2 & 3 & 2 & 3 & 4 \\
5 & 0 & 1 & 3 & 4 & 5 & 0 & 1 & 3 & 4 \\
5 & 0 & 1 & 3 & 0 & 1 & 0 & 1 & 2 & 4 \\
3 & 0 & 1 & 5 & 6 & 2 & 3 & 4 & 5 & 6 \\
2 & 3 & 4 & 5 & 0 & 1 & 6 & 3 & 2 & 4 \\
2 & 6 & 4 & 5 & 0 & 1 & 6 & 4 & 2 & 4
\end{tabular}

The winning positions within 500 are as follows.

$\begin{array}{rrrrrrrrrrrrrrr}0 & 2 & 5 & 7 & 10 & 12 & 15 & 17 & 20 & 22 & 34 & 39 & 44 & 52 & 57 \\ 62 & 65 & 67 & 72 & 85 & 95 & 109 & 119 & 124 & 127 & 130 & 132 & 137 & 142 & 147 \\ 150 & 170 & 177 & 180 & 182 & 187 & 192 & 197 & 204 & 210 & 215 & 238 & 243 & 249 & 255 \\ 257 & 260 & 262 & 267 & 272 & 275 & 312 & 317 & 322 & 327 & 332 & 335 & 340 & 345 & 350 \\ 369 & 377 & 390 & 392 & 397 & 425 & 430 & 437 & 442 & 447 & 449 & 464 & & & \end{array}$




\section{Subtraction of aliquot parts}

Two players start with a positive integer and alternately subtract any aliquot part (divisor) with the exception of the number itself from the number left by the opponent. Winner is the last player able to perform such a subtraction.

By way of example, if the original number is 12 , first player may subtract either $1,2,3,4$, or 6 (but not 12 ). If he subtract 2, leaving 10 , second player may subtract either 1, 2, or 5 .

The first 100 terms of the Sprague-Grundy sequence are

\begin{tabular}{cccccccccc}
1 & 2 & 3 & 4 & 5 & 6 & 7 & 8 & 9 & 10 \\
\hline 0 & 1 & 0 & 2 & 0 & 1 & 0 & 3 & 0 & 1 \\
0 & 2 & 0 & 1 & 0 & 4 & 0 & 1 & 0 & 2 \\
0 & 1 & 0 & 3 & 0 & 1 & 0 & 2 & 0 & 1 \\
0 & 5 & 0 & 1 & 0 & 2 & 0 & 1 & 0 & 3 \\
0 & 1 & 0 & 2 & 0 & 1 & 0 & 4 & 0 & 1 \\
0 & 2 & 0 & 1 & 0 & 3 & 0 & 1 & 0 & 2 \\
0 & 1 & 0 & 6 & 0 & 1 & 0 & 2 & 0 & 1 \\
0 & 3 & 0 & 1 & 0 & 2 & 0 & 1 & 0 & 4 \\
0 & 1 & 0 & 2 & 0 & 1 & 0 & 3 & 0 & 1 \\
0 & 2 & 0 & 1 & 0 & 5 & 0 & 1 & 0 & 2
\end{tabular}

The winning positions within 500 are as follows.

$\begin{array}{rrrrrrrrrrrrrrr}0 & 1 & 3 & 5 & 7 & 9 & 11 & 13 & 15 & 17 & 19 & 21 & 23 & 25 & 27 \\ 29 & 31 & 33 & 35 & 37 & 39 & 41 & 43 & 45 & 47 & 49 & 51 & 53 & 55 & 57 \\ 59 & 61 & 63 & 65 & 67 & 69 & 71 & 73 & 75 & 77 & 79 & 81 & 83 & 85 & 87 \\ 89 & 91 & 93 & 95 & 97 & 99 & 101 & 103 & 105 & 107 & 109 & 111 & 113 & 115 & 117 \\ 119 & 121 & 123 & 125 & 127 & 129 & 131 & 133 & 135 & 137 & 139 & 141 & 143 & 145 & 147 \\ 149 & 151 & 153 & 155 & 157 & 159 & 161 & 163 & 165 & 167 & 169 & 171 & 173 & 175 & 177 \\ 179 & 181 & 183 & 185 & 187 & 189 & 191 & 193 & 195 & 197 & 199 & 201 & 203 & 205 & 207 \\ 209 & 211 & 213 & 215 & 217 & 219 & 221 & 223 & 225 & 227 & 229 & 231 & 233 & 235 & 237 \\ 239 & 241 & 243 & 245 & 247 & 249 & 251 & 253 & 255 & 257 & 259 & 261 & 263 & 265 & 267 \\ 269 & 271 & 273 & 275 & 277 & 279 & 281 & 283 & 285 & 287 & 289 & 291 & 293 & 295 & 297 \\ 299 & 301 & 303 & 305 & 307 & 309 & 311 & 313 & 315 & 317 & 319 & 321 & 323 & 325 & 327 \\ 329 & 331 & 333 & 335 & 337 & 339 & 341 & 343 & 345 & 347 & 349 & 351 & 353 & 355 & 357 \\ 359 & 361 & 363 & 365 & 367 & 369 & 371 & 373 & 375 & 377 & 379 & 381 & 383 & 385 & 387 \\ 389 & 391 & 393 & 395 & 397 & 399 & 401 & 403 & 405 & 407 & 409 & 411 & 413 & 415 & 417 \\ 419 & 421 & 423 & 425 & 427 & 429 & 431 & 433 & 435 & 437 & 439 & 441 & 443 & 445 & 447 \\ 449 & 451 & 453 & 455 & 457 & 459 & 461 & 463 & 465 & 467 & 469 & 471 & 473 & 475 & 477 \\ 479 & 481 & 483 & 485 & 487 & 489 & 491 & 493 & 495 & 497 & 499 & & & & \end{array}$




\section{Subtraction of proper divisors}

The rules are the same with the exception that only proper divisors may be subtracted. Consider 1 an improper divisor.

The first 100 terms of the Sprague-Grundy sequence are

\begin{tabular}{cccccccccc}
1 & 2 & 3 & 4 & 5 & 6 & 7 & 8 & 9 & 10 \\
\hline 0 & 0 & 0 & 1 & 0 & 2 & 0 & 0 & 0 & 1 \\
0 & 3 & 0 & 1 & 0 & 2 & 0 & 1 & 0 & 3 \\
0 & 1 & 0 & 4 & 0 & 1 & 0 & 2 & 0 & 1 \\
0 & 0 & 0 & 1 & 0 & 2 & 0 & 1 & 0 & 4 \\
0 & 1 & 0 & 2 & 0 & 1 & 0 & 3 & 0 & 1 \\
0 & 2 & 0 & 1 & 0 & 4 & 0 & 1 & 0 & 2 \\
0 & 1 & 0 & 5 & 0 & 1 & 0 & 2 & 0 & 1 \\
0 & 4 & 0 & 1 & 0 & 2 & 0 & 1 & 0 & 3 \\
0 & 1 & 0 & 2 & 0 & 1 & 0 & 4 & 0 & 1 \\
0 & 2 & 0 & 1 & 0 & 6 & 0 & 1 & 0 & 2
\end{tabular}

The winning positions within 500 are as follows.

$\begin{array}{rrrrrrrrrrrrrrr}0 & 1 & 2 & 3 & 5 & 7 & 8 & 9 & 11 & 13 & 15 & 17 & 19 & 21 & 23 \\ 25 & 27 & 29 & 31 & 32 & 33 & 35 & 37 & 39 & 41 & 43 & 45 & 47 & 49 & 51 \\ 53 & 55 & 57 & 59 & 61 & 63 & 65 & 67 & 69 & 71 & 73 & 75 & 77 & 79 & 81 \\ 83 & 85 & 87 & 89 & 91 & 93 & 95 & 97 & 99 & 101 & 103 & 105 & 107 & 109 & 111 \\ 113 & 115 & 117 & 119 & 121 & 123 & 125 & 127 & 128 & 129 & 131 & 133 & 135 & 137 & 139 \\ 141 & 143 & 145 & 147 & 149 & 151 & 153 & 155 & 157 & 159 & 161 & 163 & 165 & 167 & 169 \\ 171 & 173 & 175 & 177 & 179 & 181 & 183 & 185 & 187 & 189 & 191 & 193 & 195 & 197 & 199 \\ 201 & 203 & 205 & 207 & 209 & 211 & 213 & 215 & 217 & 219 & 221 & 223 & 225 & 227 & 229 \\ 231 & 233 & 235 & 237 & 239 & 241 & 243 & 245 & 247 & 249 & 251 & 253 & 255 & 257 & 259 \\ 261 & 263 & 265 & 267 & 269 & 271 & 273 & 275 & 277 & 279 & 281 & 283 & 285 & 287 & 289 \\ 291 & 293 & 295 & 297 & 299 & 301 & 303 & 305 & 307 & 309 & 311 & 313 & 315 & 317 & 319 \\ 321 & 323 & 325 & 327 & 329 & 331 & 333 & 335 & 337 & 339 & 341 & 343 & 345 & 347 & 349 \\ 351 & 353 & 355 & 357 & 359 & 361 & 363 & 365 & 367 & 369 & 371 & 373 & 375 & 377 & 379 \\ 381 & 383 & 385 & 387 & 389 & 391 & 393 & 395 & 397 & 399 & 401 & 403 & 405 & 407 & 409 \\ 411 & 413 & 415 & 417 & 419 & 421 & 423 & 425 & 427 & 429 & 431 & 433 & 435 & 437 & 439 \\ 441 & 443 & 445 & 447 & 449 & 451 & 453 & 455 & 457 & 459 & 461 & 463 & 465 & 467 & 469 \\ 471 & 473 & 475 & 477 & 479 & 481 & 483 & 485 & 487 & 489 & 491 & 493 & 495 & 497 & 499\end{array}$




\section{Subtraction of primes}

The first 100 terms of the Sprague-Grundy sequence

\begin{tabular}{cccccccccc}
1 & 2 & 3 & 4 & 5 & 6 & 7 & 8 & 9 & 10 \\
\hline 0 & 0 & 1 & 1 & 2 & 2 & 3 & 3 & 4 & 0 \\
0 & 1 & 1 & 2 & 2 & 3 & 3 & 4 & 4 & 5 \\
5 & 6 & 6 & 7 & 7 & 0 & 4 & 1 & 5 & 2 \\
6 & 3 & 4 & 7 & 0 & 0 & 1 & 1 & 2 & 2 \\
3 & 3 & 4 & 8 & 5 & 7 & 6 & 8 & 9 & 0 \\
4 & 1 & 5 & 2 & 6 & 0 & 4 & 1 & 5 & 2 \\
6 & 3 & 4 & 7 & 5 & 8 & 4 & 10 & 5 & 7 \\
6 & 8 & 4 & 7 & 5 & 8 & 6 & 10 & 9 & 7 \\
4 & 8 & 5 & 10 & 6 & 0 & 4 & 1 & 5 & 2 \\
6 & 0 & 4 & 1 & 5 & 2 & 6 & 3 & 4 & 7
\end{tabular}

The winning positions within 500 are as follows.

$\begin{array}{rrrrrrrrrrrrrrr}0 & 1 & 2 & 10 & 11 & 26 & 35 & 36 & 50 & 56 & 86 & 92 & 101 & 116 & 122 \\ 126 & 134 & 146 & 156 & 170 & 176 & 188 & 196 & 206 & 218 & 236 & 248 & 254 & 260 & 266 \\ 290 & 296 & 302 & 310 & 311 & 320 & 326 & 336 & 344 & 356 & 362 & 376 & 386 & 392 & 396 \\ 404 & 416 & 426 & 446 & 452 & 470 & 476 & 482 & 486 & 494 & & & & & \end{array}$




\subsection{The nim sum of natural numbers}

The nim sum of two nonnegative integers is the addition in their base 2 notations without carries. If we write

$$
0 \boxplus 0=0, \quad 0 \boxplus 1=1 \boxplus 0=1, \quad 1 \boxplus 1=0,
$$

then in terms of the base 2 expansions of $a$ and $b$,

$a \boxplus b=\left(a_{1} a_{2} \cdots a_{n}\right) \boxplus\left(b_{1} b_{2} \cdots b_{n}\right)=\left(a_{1} \boxplus b_{1}\right)\left(a_{2} \boxplus b_{2}\right) \cdots\left(a_{n} \boxplus b_{n}\right)$.

The nim sum is associative, commutative, and has 0 as identity element. In particular, $a \boxplus a=0$ for every natural number $a$.

Here are the nim sums of numbers $\leq 15$ :

\begin{tabular}{c|cccccccccccccccc}
$\boxplus$ & 0 & 1 & 2 & 3 & 4 & 5 & 6 & 7 & 8 & 9 & 10 & 11 & 12 & 13 & 14 & 15 \\
\hline 0 & 0 & 1 & 2 & 3 & 4 & 5 & 6 & 7 & 8 & 9 & 10 & 11 & 12 & 13 & 14 & 15 \\
1 & 1 & 0 & 3 & 2 & 5 & 4 & 7 & 6 & 9 & 8 & 11 & 10 & 13 & 12 & 15 & 14 \\
2 & 2 & 3 & 0 & 1 & 6 & 7 & 4 & 5 & 10 & 11 & 8 & 9 & 14 & 15 & 12 & 13 \\
3 & 3 & 2 & 1 & 0 & 7 & 6 & 5 & 4 & 11 & 10 & 9 & 8 & 15 & 14 & 13 & 12 \\
4 & 4 & 5 & 6 & 7 & 0 & 1 & 2 & 3 & 12 & 13 & 14 & 15 & 8 & 9 & 10 & 11 \\
5 & 5 & 4 & 7 & 6 & 1 & 0 & 3 & 2 & 13 & 12 & 15 & 14 & 9 & 8 & 11 & 10 \\
6 & 6 & 7 & 4 & 5 & 2 & 3 & 0 & 1 & 14 & 15 & 12 & 13 & 10 & 11 & 8 & 9 \\
7 & 7 & 6 & 5 & 4 & 3 & 2 & 1 & 0 & 15 & 14 & 13 & 12 & 11 & 10 & 9 & 8 \\
8 & 8 & 9 & 10 & 11 & 12 & 13 & 14 & 15 & 0 & 1 & 2 & 3 & 4 & 5 & 6 & 7 \\
9 & 9 & 8 & 11 & 10 & 13 & 12 & 15 & 14 & 1 & 0 & 3 & 2 & 5 & 4 & 7 & 6 \\
10 & 10 & 11 & 8 & 9 & 14 & 15 & 12 & 13 & 2 & 3 & 0 & 1 & 6 & 7 & 4 & 5 \\
11 & 11 & 10 & 9 & 8 & 15 & 14 & 13 & 12 & 3 & 2 & 1 & 0 & 7 & 6 & 5 & 4 \\
12 & 12 & 13 & 14 & 15 & 8 & 9 & 10 & 11 & 4 & 5 & 6 & 7 & 0 & 1 & 2 & 3 \\
13 & 13 & 12 & 15 & 14 & 9 & 8 & 11 & 10 & 5 & 4 & 7 & 6 & 1 & 0 & 3 & 2 \\
14 & 14 & 15 & 12 & 13 & 10 & 11 & 8 & 9 & 6 & 7 & 4 & 5 & 2 & 3 & 0 & 1 \\
15 & 15 & 14 & 13 & 12 & 11 & 10 & 9 & 8 & 7 & 6 & 5 & 4 & 3 & 2 & 1 & 0
\end{tabular}

Theorem 17.3. Suppose two players alternately play one of the counter games $\mathcal{G}_{1}, \ldots, \mathcal{G}_{k}$ which have Sprague-Grundy sequences $\left(g_{1}(n)\right), \ldots$, $\left(g_{k}(n)\right)$ respectively. The player who secures a position $\left(n_{1}, n_{2}, \ldots, n_{k}\right)$ with

$$
g_{1}\left(n_{1}\right) \boxplus g_{2}\left(n_{2}\right) \boxplus \cdots \boxplus g_{k}\left(n_{k}\right)=0
$$

has a winning strategy. 


\subsection{The game Nim}

Given three piles of marbles, with $a, b, c$ marbles respectively, players $A$ and $B$ alternately remove a positive amount of marbles from any pile. The player who makes the last move wins.

Theorem 17.4. In the game nim, the player who can balance the nim sum equation has a winning strategy.

Therefore, provided that the initial position $(a, b, c)$ does not satisfy $a \boxplus b \boxplus c=0$, the first player has a winning strategy. For example, suppose the initial position is $(12,7,9)$. Since $12 \boxplus 9=5$, the first player can remove 2 marbles from the second pile to maintain a balance of the nim sum equation,

$$
12 \boxplus 5 \boxplus 9=0
$$

thereby securing a winning position.

This theorem indeed generalizes to an arbitrary number of piles. 


\subsection{Northcott's variation of Nim}

Two players alternately move their counters on one of the rows, the only restriction being not moving onto or beyond the opponent's counter. The one who cannot move loses.

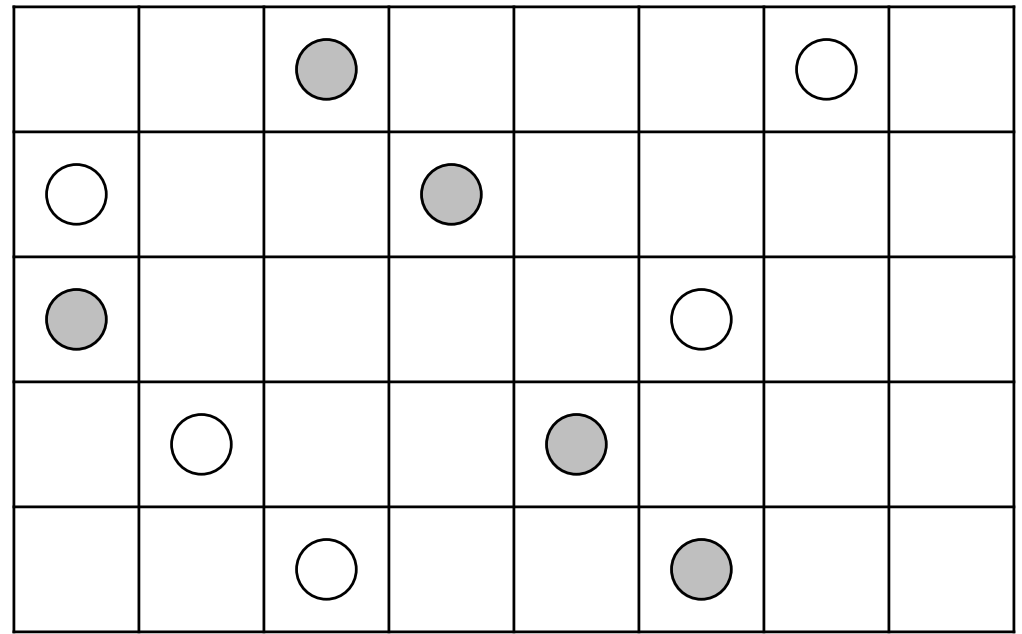

This is equivalent to nim if one considers a number of piles of counters corresponding to the number of spaces between the counters on the rows. (If a player tries to increase the number of spaces, the other player can force the same distance by pursueing the same number of spaces). Therefore the player who can balance the nim sum equation has a winning strategy.

For example, in the above arrangement, the numbers of spaces have nim sum

$3 \boxplus 2 \boxplus 4 \boxplus 2 \boxplus 2=5$.

It can be made 0 by moving 3 spaces in row 3 . 


\subsection{Wythoff's game}

Wythoff's game is a variant of Nim. Given two piles of marbles, a player either removes an arbitrary positive amount of marbles from any one pile, or an equal (positive) amount of marbles from both piles. The player who makes the last move wins.

We describe the position of the game by the amounts of marbles in the two piles.

If you can make $(2,1)$, then you will surely win no matter how your opponent moves. Now, to forbid your opponent to get to this position, you should occupy $(3,5)$.

The sequence of winning positions: starting with $\left(a_{1}, b_{1}\right)=(1,2)$, construct $\left(a_{k}, b_{k}\right)$ by setting

$$
\begin{aligned}
a_{k} & :=\min \left\{c: c>a_{i}, b_{i}, i<k\right\}, \\
b_{k} & :=a_{k}+k .
\end{aligned}
$$

Here are the 18 smallest winning positions for Wythoff's game:

$\begin{array}{llllllllllllllllll}1 & 3 & 4 & 6 & 8 & 9 & 11 & 12 & 14 & 16 & 17 & 19 & 21 & 22 & 24 & 25 & 27 & 29\end{array}$

$\begin{array}{llllllllllllllllll}2 & 5 & 7 & 10 & 13 & 15 & 18 & 20 & 23 & 26 & 28 & 31 & 34 & 36 & 39 & 41 & 44 & 47\end{array}$

Theorem 17.5. The winning positions of Wythoff's game are the pairs $\left(\lfloor n \varphi\rfloor,\left\lfloor n \varphi^{2}\right\rfloor\right)$, where $\varphi=\frac{\sqrt{5}+1}{2}$ is the golden ratio. 


\section{Appendix: Beatty's Theorem}

If $\alpha$ and $\beta$ are positive irrational numbers satisfying $\frac{1}{\alpha}+\frac{1}{\beta}=1$, then the sequences

$$
\lfloor\alpha\rfloor,\lfloor 2 \alpha\rfloor,\lfloor 3 \alpha\rfloor, \ldots
$$

and

$$
\lfloor\beta\rfloor,\lfloor 2 \beta\rfloor,\lfloor 3 \beta\rfloor, \ldots
$$

form a partition of the sequence of positive integers.

See Russian problem book, MG Problem 1300, solution, 62 (1989) 203.

If $r$ and $s$ are positive integers and $n=r+s$, then the union of the two sequences

$$
\left\lfloor\frac{n}{r}\right\rfloor,\left\lfloor\frac{2 n}{r}\right\rfloor, \ldots,\left\lfloor\frac{(r-1) n}{r}\right\rfloor
$$

and

$$
\left\lfloor\frac{n}{s}\right\rfloor,\left\lfloor\frac{2 n}{s}\right\rfloor, \ldots,\left\lfloor\frac{(s-1) n}{s}\right\rfloor .
$$

is the natural sequence $1,2, \ldots, n-2$ if and only if $r$ and $s$ are relatively prime. 


\section{Exercise}

1. What are the winning positions in the battle of numbers with $N=$ 100 and $d=7$ ?

2. The golden ratio $\varphi$ has the property that $\varphi^{2}$ and $\varphi$ have exactly the same digits after the decimal point. Find all numbers $\theta$ which have exactly the same digits as $\theta^{2}$ after the decimal point.

3. A announces a two digit number from 01 to 99 . B reverses the digits of this number and adds to it the sum of its digits and then announces his result. A continues in the same pattern. All numbers are reduced modulo 100, so that only two digit numbers are announced. What choices has $\mathrm{A}$ for the initial number in order to insure that B will at some time announce $00 ?^{2}$

\footnotetext{
2.89 Jo 08 ‘9
} 


\section{Project: Another subtraction game}

Starting with a given positive integer, two players alternately subtract numbers not more than the predecessor used. The one who gets down to zero is the winner. Under what condition does the first player have a winning strategy? 


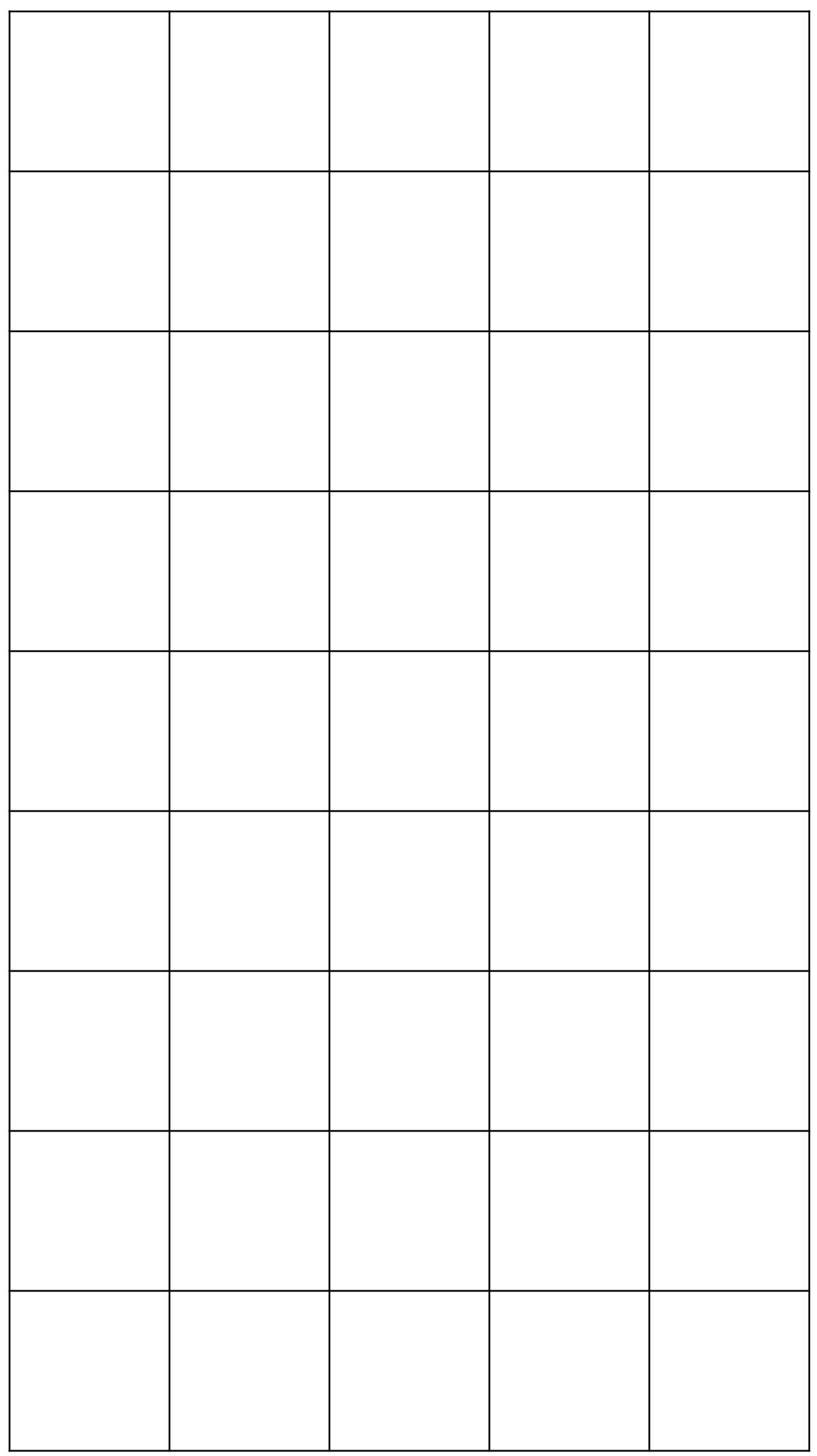




\section{Chapter 18}

\section{Repunits}

$1 \quad k$-right-transposable integers

$2 k$-left-transposable integers

3 Sam Yates' repunit riddles

4 Recurring decimals

5 The period length of a prime

Appendix: Factorizations of repunits $R_{n}, n \leq 50$

Appendix: Primitive divisors of repunits

Appendix: Multiplicative digital root

Exercise

Project: Period lengths of primes $<100$.

Project: A number whose cube root divides all numbers obtained by cyclic permutations of its digits. 


\section{1 $k$-right-transposable integers}

Let $k$ be a given positive integer. A positive integer $X$ is $k$-transposable if in moving the leftmost digit to the rightmost, the number is multiplied by $k$.

Note that $X$ is a repdigit if and only if $k=1$. We shall assume $k>1$. Suppose the number $X$ has $n$ digits, with leftmost digit $a$. We have

$$
10\left(X-a \cdot 10^{n-1}\right)+a=k X .
$$

From this, $(10-k) X=a\left(10^{n}-1\right)=9 a \cdot R_{n}$.

If $k \neq 3,10-k$ can only have prime divisors $2,3,5$. The equation will reduce to $X=$ a repdigit, which is clearly impossible.

For $k=3$, we have $7 X=a\left(10^{n}-1\right)$. If $a=7$, then again $X$ is a repdigit. Therefore, we must have 7 dividing $10^{n}-1$. This is possible only if $n$ is a multiple of 6 . Therefore $X=a \cdot \frac{10^{6 m}-1}{7}$ and has first digit a.

Now,

$$
\frac{10^{6 m}-1}{7}=(142857)_{m}
$$

It is easy to see that $a$ can only be 1 or 2 .

Therefore, the only $k$-transposable numbers are $(142857)_{m}$ and $(285714)_{m}$ with $k=3$. 


\section{$18.2 k$-left-transposable integers}

Let $k$ be a given positive integer. A positive integer $X$ is $k$-left-transposable if in moving the rightmost digit to the leftmost, the number is multiplied by $k$. Note that $X$ is a repdigit if and only if $k=1$. We shall assume $k>1$. Suppose the number $X$ has $n$ digits, and its rightmost digit is $b$. We have

$$
b \cdot 10^{n-1}+\frac{X-b}{10}=k X .
$$

From this, $b \cdot 10^{n}+X-b=10 k X$, and

$$
(10 k-1) X=b\left(10^{n}-1\right) .
$$

Since $X$ is has $n$ digits, $b\left(10^{n}-1\right)>(10 k-1) 10^{n-1}$, and $b>\frac{10^{n-1}(10 k-1)}{10^{n}-1}=$ $k-\frac{1}{10}$. This shows that $b \geq k$.

\begin{tabular}{ccc}
$k$ & & $n$ \\
\hline \hline 2 & $19 X=b\left(10^{n}-1\right)$ & $18 m$ \\
3 & $29 X=b\left(10^{n}-1\right)$ & $28 m$ \\
4 & $39 X=b\left(10^{n}-1\right)$ & $12 m$ \\
5 & $49 X=b\left(10^{n}-1\right)$ & $42 m$ \\
6 & $59 X=b\left(10^{n}-1\right)$ & $58 m$ \\
7 & $69 X=b\left(10^{n}-1\right)$ & $22 m$ \\
8 & $79 X=b\left(10^{n}-1\right)$ & $13 m$ \\
9 & $89 X=b\left(10^{n}-1\right)$ & $44 m$
\end{tabular}

These lead to the following numbers:

$$
\begin{aligned}
& X_{2}=105263157894736842, \\
& X_{3}=1034482758620689655172413793, \\
& X_{4}=102564102564, \\
& X_{5}=102040816326530612244897959183673469387755, \\
& X_{6}=1016949152542372881355932203389830508474576271186440677966, \\
& X_{7}=1014492753623188405797, \\
& X_{8}=1012658227848, \\
& X_{9}=10112359550561797752808988764044943820224719 .
\end{aligned}
$$

Each of these $X_{k}$ can be replaced by $\frac{b}{k} \cdot X_{k}$ for $k=b, \ldots, 9$. Every $k$-left-transposable number is of the form $\left(X_{k}\right)_{m}$ for $X_{k}$ given above and $m \geq 1$. 


\subsection{Sam Yates' repunit riddles}

1. What digits should be substituted for the letters so that the sum of the nine identical addends will be a repunit? ${ }^{1}$

\begin{tabular}{|c|c|c|c|c|c|c|c|c|}
\hline & $R$ & $E$ & $P$ & $U$ & $N$ & $I$ & $T$ & $S$ \\
\hline & $R$ & $E$ & $P$ & $U$ & $N$ & $I$ & $T$ & $S$ \\
\hline & $R$ & $E$ & $P$ & $U$ & $N$ & $I$ & $T$ & $S$ \\
\hline & $R$ & $E$ & $P$ & $U$ & $N$ & $I$ & $T$ & $S$ \\
\hline & $R$ & $E$ & $P$ & $U$ & $N$ & $I$ & $T$ & $S$ \\
\hline & $R$ & $E$ & $P$ & $U$ & $N$ & $I$ & $T$ & $S$ \\
\hline & $R$ & $E$ & $P$ & $U$ & $N$ & $I$ & $T$ & $S$ \\
\hline & $R$ & $E$ & $P$ & $U$ & $N$ & $I$ & $T$ & 5 \\
\hline & $R$ & $E$ & $P$ & $U$ & $N$ & $I$ & $T$ & $S$ \\
\hline
\end{tabular}

2. Are two repunits with consecutive even numbers as their subscripts relatively prime? ${ }^{2}$

3. Are two repunits with consecutive numbers as their subscripts relatively prime? ${ }^{3}$

4. Are two repunits with consecutive odd numbers as their subscripts relatively prime? ${ }^{4}$

5. Aside from 3 , what prime $p$ divides $R_{p}{ }^{5}$

6. What digit does each letter of this multiplication represent? ${ }^{6}$

$$
\begin{aligned}
& \begin{array}{lllllll}
R & R & R & R & R & R & R
\end{array}
\end{aligned}
$$

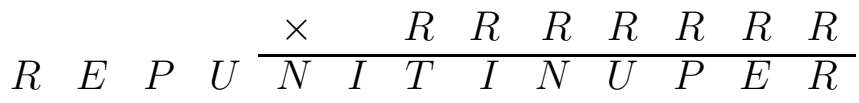

7. An old car dealer's record in the 1960's shows that the total receipts for the sale of new cars in one year came to 1,111,111.00 dollars. If each car had eight cylinders and was sold for the same price as each other car, how many cars did he sell?

\footnotetext{
${ }^{1}$ REPUNITS $=12345679$ and the sum is $R_{9}$.

${ }^{2}$ No. Repunits with even subscripts are divisible by 11 .

${ }^{3}$ Yes. Consecutive numbers are relatively prime, and $\operatorname{gcd}\left(R_{m}, R_{n}\right)=R_{\operatorname{gcd}(m, n)}$.

${ }^{4}$ Yes. Consecutive odd numbers are relatively prime.

5 כuON.

${ }^{6} R_{7} \times R_{7}=R_{13}$.
} 
8. Find a pair of repunits whose product is a 100-digit palindrome. ${ }^{7}$

9. If a Mersenne number $M_{p}=2^{p}-1$ is prime, is the corresponding repunit $R_{p}$ also prime? ${ }^{8}$

10. What is the smallest repunit divisible by the square of 11 ? What is the smallest repunit divisible by the square of $R_{11}$ ?

In general, the smallest repunit divisible by the square of $R_{n}$ is $R_{N}$, where $N=n R_{n}$.

${ }^{7}$ If $R_{p} \cdot R_{q}$ has 100 digits, $p+q=101$. Suppose $p \leq q$ and $p=9 k+m$ for $1 \leq m \leq 9$. Since the product is a palindrome, it cannot contain $\mathbf{A}$ and $\mathbf{B}$. We must have $k=0$ and $p \leq 9$. For any $p=2, \ldots, 9$, the product $R_{p} \cdot R_{q}$ is the palindrome $\overline{\mathbf{p}}_{102-2 p} 1$, where

$$
\overline{\mathbf{p}}=\left\{\begin{array}{ll}
12_{k} & \text { if } p=2 \\
12 \cdots(p-1)(p-1) 32 & \text { if } 3 \leq p \leq 9 .
\end{array} .\right.
$$

${ }^{8} M_{3}=2^{3}-1=7$ is prime but $R_{3}=111=3 \times 37$. 


\subsection{Recurring decimals}

The decimal expansion of a rational number is eventually periodic. It is finite if and only if the denominator has no prime divisors other than 2 and 5.

The decimal expansion of $\frac{1}{n}$ is purely periodic if and only if $n$ is a prime. For $p=3$, this is the most well known recurring decimal

$$
\frac{1}{3}=0.333 \cdots=0 . \overline{3} \text {. }
$$

with period length 1 . Here are the periods of the reciprocals of the first few primes, together with their period lengths.

\begin{tabular}{lll}
$p$ & period & $\lambda(p)$ \\
\hline 3 & 3 & 1 \\
7 & 142857 & 6 \\
11 & 09 & 2 \\
13 & 076923 & 6 \\
17 & 0588235294117647 & 16 \\
19 & 052631578947368421 & 18 \\
23 & 0434782608695652173913 & 22 \\
29 & 0344827586206896551724137931 & 28 \\
31 & 032258064516129 & 15 \\
37 & 027 & 3 \\
41 & 0243902439 & 10 \\
43 & 023255813953488372093 & 21 \\
47 & 0212765957446808510638297872340425531914893617 & 46
\end{tabular}




\subsection{The period length of a prime}

The period length of a prime number $p$ means the length of the shortest repeating block of digits in the decimal expansion of $\frac{1}{p}$.

Suppose $\frac{1}{p}$ has period length $\lambda$ :

$$
\frac{1}{p}=0 . \overline{a_{1} \cdots a_{\lambda}}
$$

Then moving the decimal places $n$ places to the right, we have

$$
\frac{10^{\lambda}}{p}=a_{1} \cdots a_{\lambda} \cdot \overline{a_{1} \cdots a_{\lambda}}
$$

and

$$
\frac{10^{\lambda}}{p}-\frac{1}{p}=a_{1} \cdots a_{\lambda}
$$

is an integer. This means that $p$ divides $10^{\lambda}-1$. Clearly, $p$ cannot be 2 or 5. It is known that if $p \neq 2,5$, then $10^{p-1}-1$ is divisible by $p$, and any number $\lambda$ for which $10^{\lambda}-1$ is divisible by $p$ divides $p-1$.

Theorem 18.1. If $p \neq 2,5$, the period length of $p$ is the smallest divisor $\lambda$ of $p-1$ such that $p$ divides $10^{\lambda}-1$.

Theorem 18.2. Let $p>5$ be a prime. The period length of $p$ is the smallest divisor $n$ of $p-1$ such that $p$ divides $R_{n}$.

Proof. Note that $10^{n}-1=9_{n}=9 \times R_{n}$. If $p \neq 3$, then $p$ divides $R_{n}$.

We say that $p$ is a primitive prime divisor the repunit $n$. Thus, for a prime $p>5$, the period length of $p$ is the number $n$ for which $p$ is primitive prime divisor. A table of primitive prime divisors of repunits is given in an Appendix. 


\section{Appendix: Factorizations of repunits $R_{n}$ for $n \leq 50$}

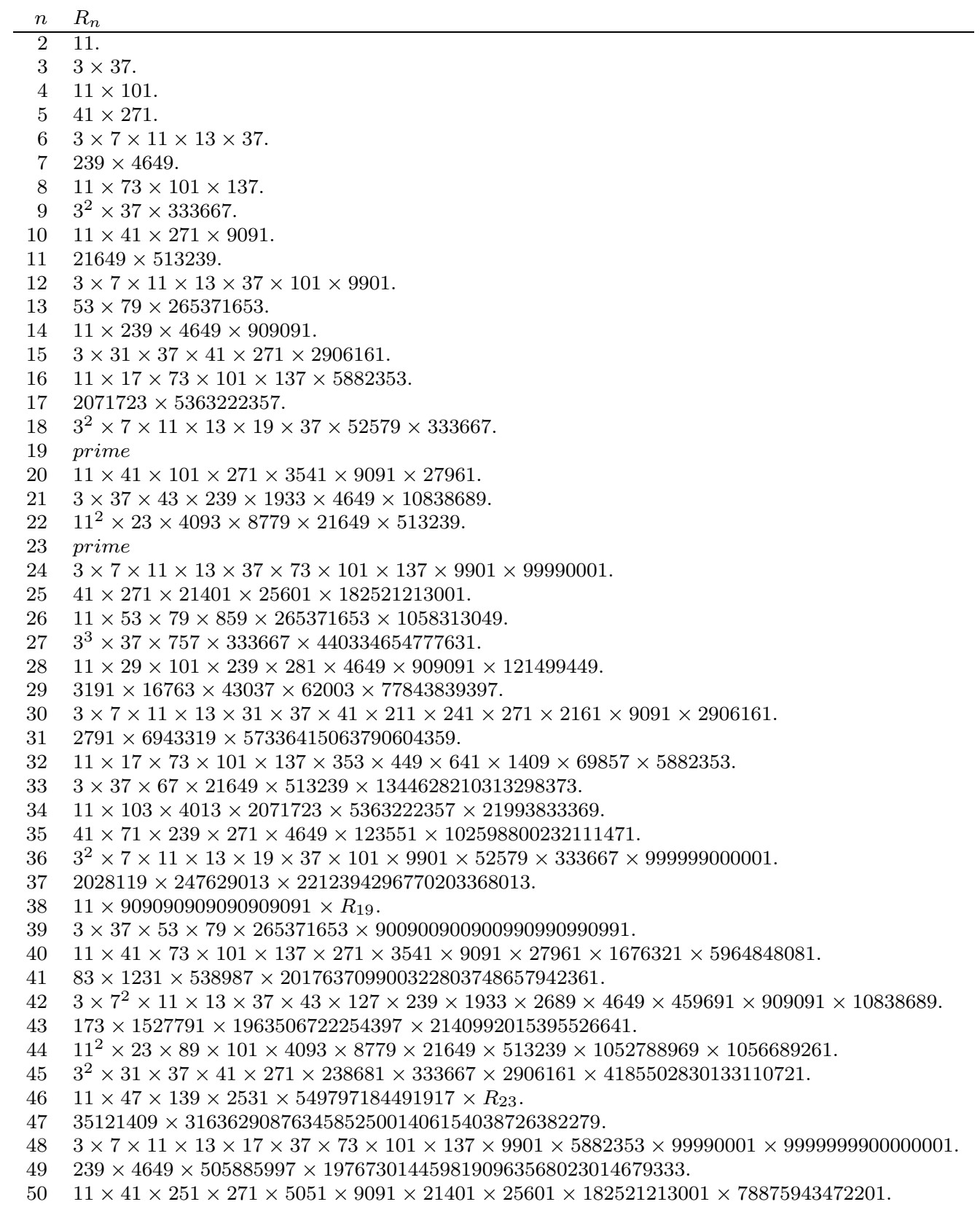




\section{Appendix: Primitive prime divisors of repunits}

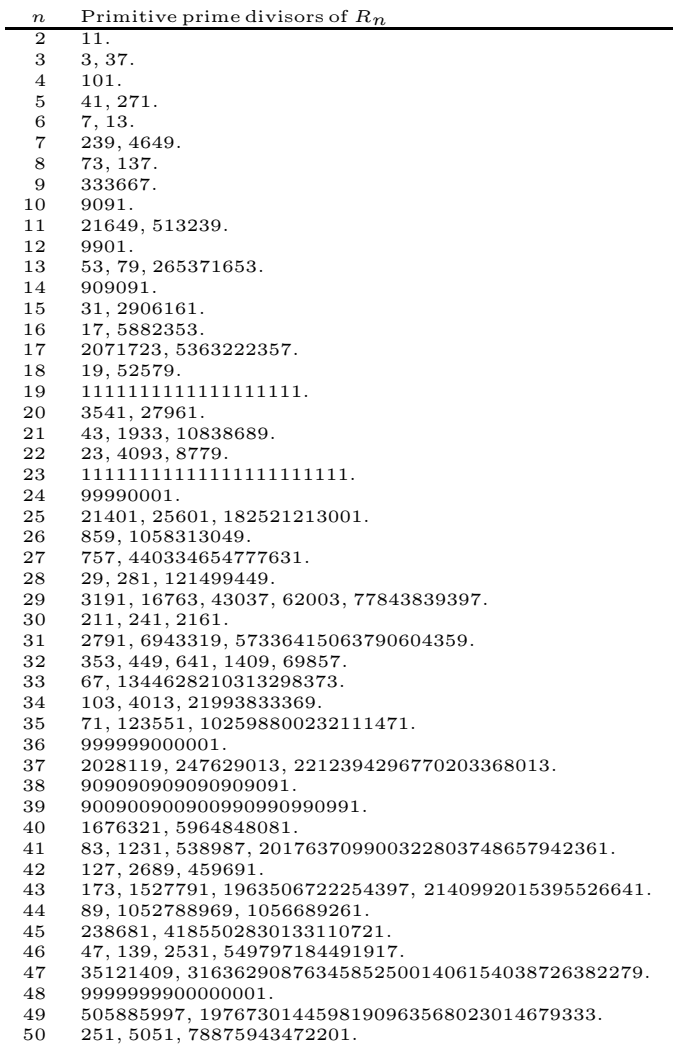

\section{Exercise}

Find all prime numbers $p$ for which $\frac{1}{p}$ has period length 11.9

\footnotetext{
${ }^{9}$ Problem 2207, J. Recreational Math., 27 (1995) 59.
} 


\section{Appendix: The multiplicative digital root}

The multiplicative digital root of a number is the single digit number obtained by iterating the digital product operation. Thus,

$$
6244 \rightarrow 6 \cdot 2 \cdot 4 \cdot 4=192 \rightarrow 1 \cdot 9 \cdot 2=18 \rightarrow 1 \operatorname{cdot} 8=8 .
$$

Theorem 18.3 (Kuczma). The multiplicative digital root of $n$ is 1 if and only if $n$ is a repunit.

Proof. The digital product of a number is 1 if and only if it is a repunit. We claim that no number can have a repunit $R_{n}, n \geq 2$, for digital product. If so, this would mean that $R_{n}$ factors into one digit numbers, and its prime divisors can only be 3 and 7 . In other words, $R_{n}=3^{h} \cdot 7^{k}$ for some integers $h, k \geq 0$.

The powers of 7 modulo 100 are 1, 7, 49, 43. Those of 3 modulo 100 are given in the first row of the table below. Their product cannot be 11 modulo 100.

$$
\begin{array}{cccccccccccccccccccc}
1 & 3 & 9 & 27 & 81 & 43 & 29 & 87 & 61 & 83 & 49 & 47 & 41 & 23 & 69 & 7 & 21 & 63 & 89 & 67 \\
7 & 21 & 63 & 89 & 67 & 1 & 3 & 9 & 27 & 81 & 43 & 29 & 87 & 61 & 83 & 49 & 47 & 41 & 23 & 69 \\
49 & 47 & 41 & 23 & 69 & 7 & 21 & 63 & 89 & 67 & 1 & 3 & 9 & 27 & 81 & 43 & 29 & 87 & 61 & 83 \\
43 & 29 & 87 & 61 & 83 & 49 & 47 & 41 & 23 & 69 & 7 & 21 & 63 & 89 & 67 & 1 & 3 & 9 & 27 & 813
\end{array}
$$




\section{Exercise}

1. What are the decimal expansions of $\frac{1}{2^{k}}, \frac{1}{5^{k}}$, and $\frac{1}{2^{h} \cdot 5^{k}}$ ?

2. Calculate the period of $p=67$.

3. If $p$ is any odd prime, show that the decimal expansion of the fraction $\frac{1}{p}$ will repeat in $\frac{p-1}{2}$ digits or some factor thereof it and only if $p \equiv \pm 3^{k}(\bmod 40)$.

4. What is the smallest integer of $2 n$ identical digits which is the product of two $n$-digit numbers?

Clearly $n \geq 2$. Since $1111=11 \times 101$, we seek 6 digit numbers. Now,

$$
R_{6}=3 \times 7 \times 11 \times 13 \times 37 .
$$

There are four ways of rearranging it as a product of two 3-digit numbers:

$$
143 \times 777=231 \times 481=259 \times 429=273 \times 407 .
$$

5. Let $N$ be an integer of $p$ digits. If the last digit is removed and placed before the remaining $p-1$ digits, a new number of $p$ digits is formed which is $\frac{1}{n}$ th of the original number. Find the most general such number $N$.

6. (a) Find the smallest integer $N$ such that, if the units digits is transposed from right to left, a number $M$ is obtained where $M=5 N .{ }^{10}$

7. A certain 3-digit number yields a quotient of 26 when divided by the sum of its digits. If the digits are reversed, the quotient is 48 . What is the smallest 3-digit number for which this is possible?

8. Without the use of tables, find the smallest integer whose cube terminates in seven sevens. ${ }^{11}$ 
Project: Period lengths of primes $<100$

Find the period lengths of the primes $<100$ by using the factorization of repunits given above. Note that 59,61, and 97 are the only primes $<100$ that cannot be found in the table above.

\begin{tabular}{|c|c|c|c|c|}
\hline Prime & Period lengths & & Prime & Period lengths \\
\hline \hline 7 & & & 11 & \\
\hline 13 & & & 17 & \\
\hline 19 & & & 23 & \\
\hline 29 & & & 31 & \\
\hline 37 & & & 41 & \\
\hline 43 & & & 47 & \\
\hline 53 & & & 59 & \\
\hline 61 & & & 67 & \\
\hline 71 & & & 73 & \\
\hline 79 & & & 83 & \\
\hline 89 & & & 97 & \\
\hline
\end{tabular}




\section{Project}

It is known that the number

$$
N=19000458461599776807277716631
$$

is a perfect cube. Find its cube root $M$. Verify that $N=N_{0}$ the twentyeight numbers $N_{i}, 1 \leq i \leq 28$ which are formed by cyclic permutations of its digits are all divisible by the cube root of $N$.

\begin{tabular}{|r|r|l|}
\hline$i$ & $N_{i}$ & $N_{i} \div M$ \\
\hline \hline 0 & 19000458461599776807277716631 & \\
\hline 1 & 90004584615997768072777166311 & \\
\hline 2 & 45846159977680727771663119 & \\
\hline 3 & 458461599776807277716631190 & \\
\hline 4 & 4584615997768072777166311900 & \\
\hline 5 & 45846159977680727771663119000 & \\
\hline 6 & 58461599776807277716631190004 & \\
\hline 7 & 84615997768072777166311900045 & \\
\hline 8 & 46159977680727771663119000458 & \\
\hline 9 & 61599776807277716631190004584 & \\
\hline 10 & 15997768072777166311900045846 & \\
\hline 11 & 59977680727771663119000458461 & \\
\hline 12 & 99776807277716631190004584615 & \\
\hline 13 & 97768072777166311900045846159 & \\
\hline 14 & 77680727771663119000458461599 & \\
\hline 15 & 76807277716631190004584615997 & \\
\hline 16 & 68072777166311900045846159977 & \\
\hline 17 & 80727771663119000458461599776 & \\
\hline 18 & 7277716631190004584615997768 & \\
\hline 19 & 72777166311900045846159977680 & \\
\hline 20 & 27771663119000458461599776807 & \\
\hline 21 & 77716631190004584615997768072 & \\
\hline 22 & 77166311900045846159977680727 & \\
\hline 23 & 71663119000458461599776807277 & \\
\hline 24 & 16631190004584615997768072777 & \\
\hline 25 & 66311900045846159977680727771 & \\
\hline 26 & 63119000458461599776807277716 & \\
\hline 27 & 31190004584615997768072777166 & \\
\hline 28 & 11900045846159977680727771663 & \\
\hline & & \\
\hline
\end{tabular}




\section{Chapter 19}

\section{More digital trivia}

1. Larry, Curly, and Moe had an unusual combination of ages. The sum of any two of the three ages was the reverse of the third age (e.g., $16+52=68$, the reverse of 86). All were under 100 years old.

(a) What was the sum of the ages?

(b) If Larry was older than either of the others, what was the youngest he could be?

2. Let $n$ be a nonnegative integer. The number formed by placing $2^{n}$ and $2^{n+1}$ side by side in any order is divisible by 3 .

3. Find positive integers $x, y, z$ (less than 100), such that $x^{2}+y^{2}=$ $z^{2}$ and $X^{2}+Y^{2}=Z^{2}$ where $X, Y, Z$ are derived from $x, y, z$ by inserting an extra digit (the same for all) on the left.

4. (a) Find the smallest positive integer $N$ having the property that the sum of its digits does not divide the sum of the cubes of its digits.

(b) Find the two consecutive positive integers each of which equals the sum of the cubes of its digits. ${ }^{2}$

5. Find two perfect cubes which, considered jointly, contain the digits $0,1,2,3,4,5,6,7,8$ and 9 each once. Is the solution unique ? ${ }^{3}$

6. Show that there is but one five-digit integer whose last three digits are alike and whose square contains no duplicate digits. ${ }^{4}$

\footnotetext{
${ }^{1}$ Suppose $2^{n}$ has $k$ digits. Putting $2^{n+1}$ on the left hand side of $2^{n}$ gives the number $2^{n+1} \cdot 10^{k}+2^{n}$. Modulo 3, this is $(-1)^{n+1}+(-1)^{n} \equiv 0$

${ }^{2 \cdot}$ ILE pur $0 \angle \mathcal{E}(\mathrm{q}): \mathrm{ZII}=N$ (e)

${ }^{3}$ Unique solution: $29 \& \mp 08={ }_{\varepsilon} \& 6$ pur L9Z6 $={ }_{\varepsilon} \mathrm{IZ}$

${ }^{4}$ '78Z\&L0L699 $={ }_{7}$ ZZZL8
} 
7. Determine the largest and smallest perfect squares which can be written with the ten digits $0,1,2,3,4,5,6,7,8,9$, used once each in both cases.

8. Find two perfect squares, of five digits each, which together contain all the digits $0,1,2,3,4,5,6,7,8,9$. How many solutions are there ?

9. Let $k \geq 3$. Determine all numbers of the form $1^{k}+2^{k}+\cdots+n^{k}$ whose decimal expansions consist of the ten digits $0,1,2,3,4,5$, $6,7,8$, and 9 without repetition.

10. Determine all binomial coefficients whose decimal expansions consist of the ten digits $0,1,2,3,4,5,6,7,8$, and 9 without repetition. 5

11. Find a number whose cube and fourth power together contain the ten digits, once each. (See E116 and Problem following E602). 6

12. Find a multiple of 7 whose square has eight digits of the form ababbbcc. ${ }^{7}$

13. What is the largest prime whose square contains no duplicate digits $?^{8}$

14. Find a four-digit square which remains a square when two zeros are intercalated between the thousands digit and the hundreds digit. ${ }^{9}$

15. Find a number and its fourth power, which together have nine digits, all different. ${ }^{10}$

16. Find a perfect square whose digits form one of the permutations of five consecutive digits. (See also E538, 578). ${ }^{11}$

17. Find a perfect square of 7 digits with all digits even and positive. 12

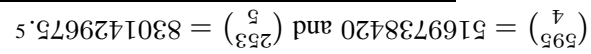

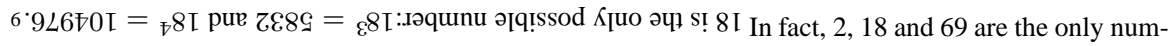
bers with two distinct powers which together contain the ten digits, once each.

7 TELLLSLS $={ }_{7} 889 L$, even without restriction to multiples of 7 .

${ }_{8} \cdot 609$ LE8L9T $={ }_{Z} L 6 \&$ LZ

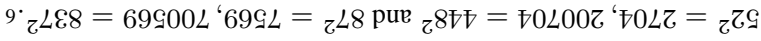

$10 \cdot 9 \angle 9870 \mathrm{I}={ }_{\tau} u$ pue ${ }^{\prime} \textrm{ } Z \varepsilon=u$

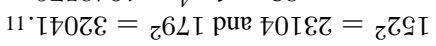

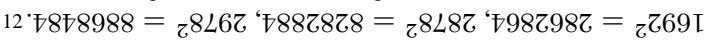


18. Find a number of the form $a b 0 c d$ whose square contains the nine digits $1,2,3,4,5,6,7,8,9$. ${ }^{13}$

19. Find a perfect cube whose digits form a permutation of consecutive digits. (See also E538). ${ }^{14}$

20. Find a square of ten digits such that the two numbers formed by the first five and last five digits are consecutive. ${ }^{15}$

21. Find the smallest four-digit number such that the sum of products of pairs of digits is equal to the sum of products of sets of three.

22. Find three three-digit numbers in geometrical progression which can be derived from one another by cyclic permutation of digits. (See also E714).

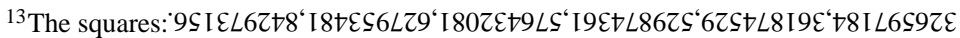

$14{ }^{2} L Z \mp S 9 \& 8={ }_{\varepsilon} \& 0 Z$ The only higher powers satisfying the same condition are the fifth powers of 32 and 243.

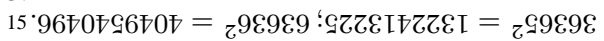




\section{Chapter 20}

\section{The shoemaker's knife}

1 Archimedes' twin circles

2 Incircle of the shoemaker's knife

Archimedes' construction

Bankoff's constructions

Woo's constructions

3 More Archimedean circles

Exercise

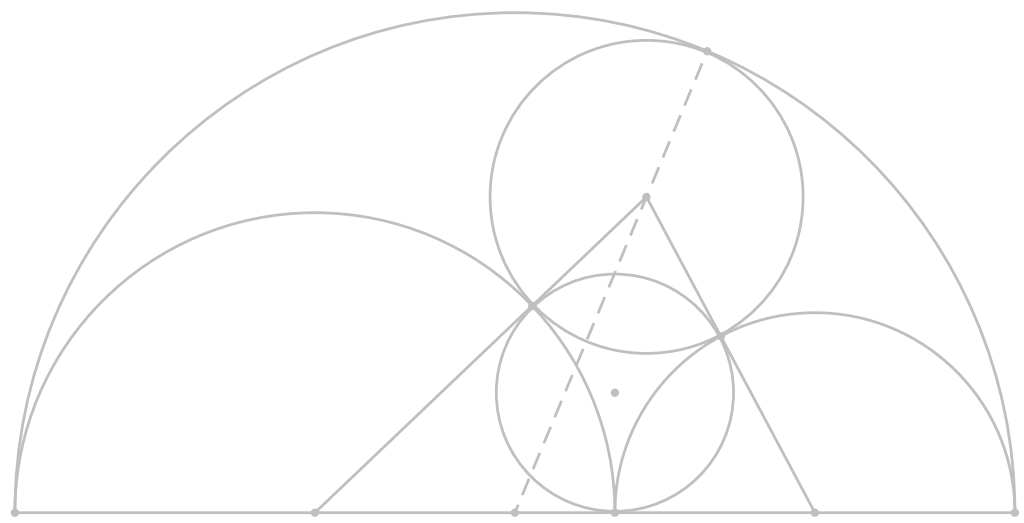




\subsection{Archimedes' twin circles}

Let $P$ be a point on a segment $A B$. The region bounded by the three semicircles (on the same side of $A B$ ) with diameters $A B, A P$ and $P B$ is called a shoemaker's knife. Suppose the smaller semicircles have radii $a$ and $b$ respectively. Let $Q$ be the intersection of the largest semicircle with the perpendicular through $P$ to $A B$. This perpendicular is an internal common tangent of the smaller semicircles.
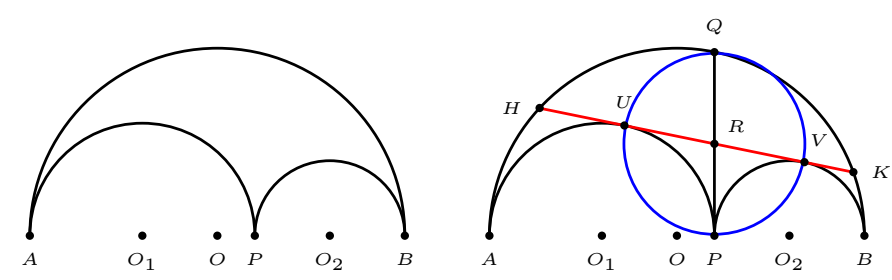

Theorem 20.1 (Archimedes). The two circles each tangent to $C P$, the largest semicircle $A B$ and one of the smaller semicircles have equal radii $t$, given by

$$
t=\frac{a b}{a+b}
$$
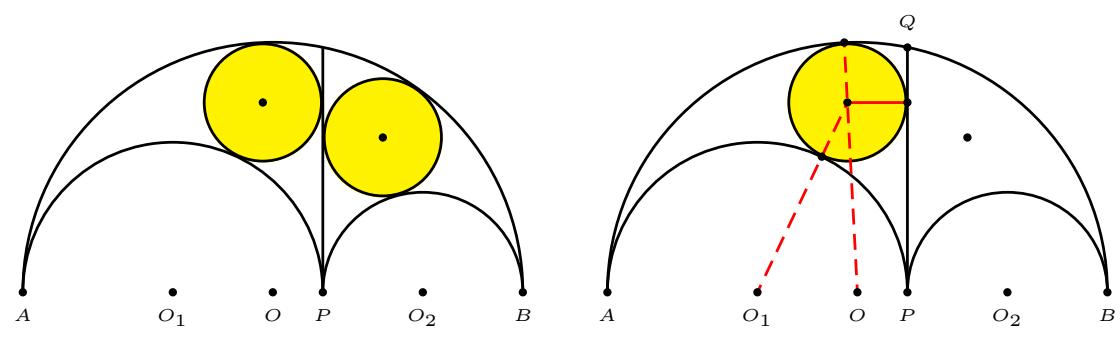


\subsection{Incircle of the shoemaker's knife}

\subsubsection{Archimedes' construction}

Theorem 20.2 (Archimedes). The circle tangent to each of the three semicircles has radius given by

$$
\rho=\frac{a b(a+b)}{a^{2}+a b+b^{2}} .
$$

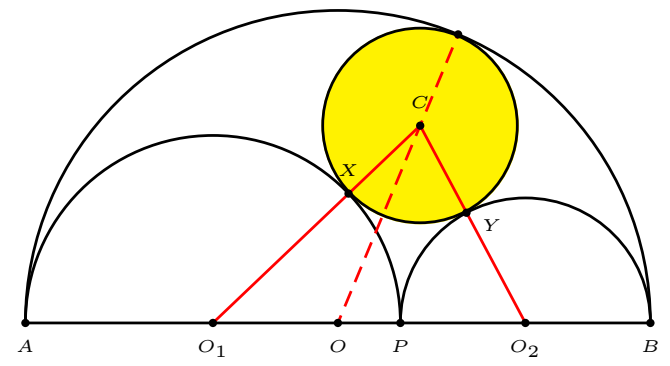

\section{Construction}

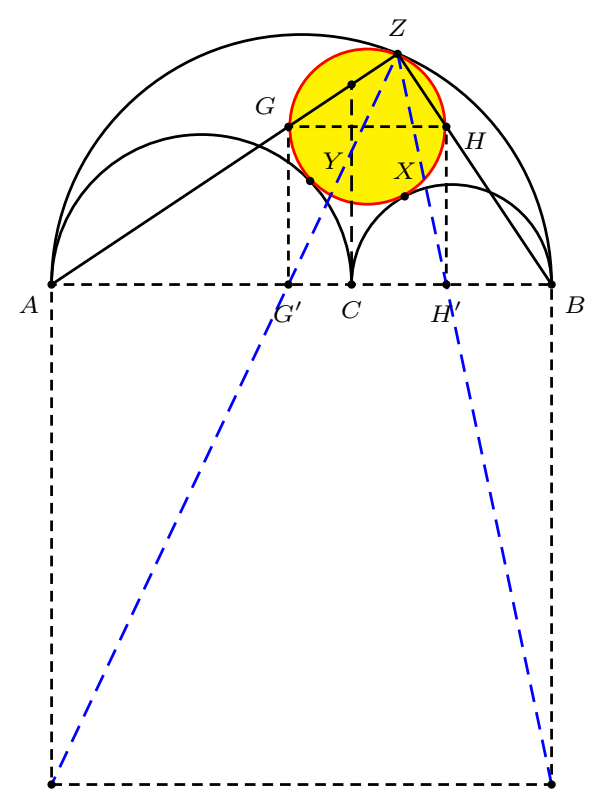




\subsubsection{Bankoff's constructions}

Theorem 20.3 (Leon Bankoff). If the incircle $C(\rho)$ of the shoemaker's knife touches the smaller semicircles at $X$ and $Y$, then the circle through the points $P, X, Y$ has the same radius as the Archimedean circles.

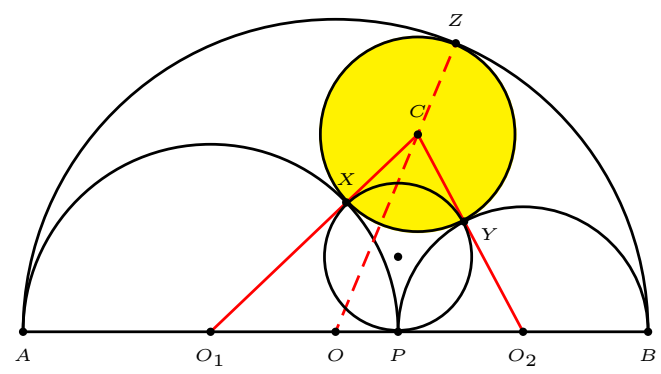

Proof. The circle through $P, X, Y$ is clearly the incircle of the triangle $\mathrm{CO}_{1} \mathrm{O}_{2}$, since

$$
C X=C Y=\rho, \quad O_{1} X=O_{1} P=a, \quad O_{2} Y=O_{2} P=b .
$$

The semiperimeter of the triangle $\mathrm{CO}_{1} \mathrm{O}_{2}$ is

$$
a+b+\rho=(a+b)+\frac{a b(a+b)}{a^{2}+a b+b^{2}}=\frac{(a+b)^{3}}{a^{2}+a b+b^{2}} .
$$

The inradius of the triangle is given by

$$
\sqrt{\frac{a b \rho}{a+b+\rho}}=\sqrt{\frac{a b \cdot a b(a+b)}{(a+b)^{3}}}=\frac{a b}{a+b} .
$$

This is the same as $t$, the common radius of Archimedes' twin circles.

\section{First construction}

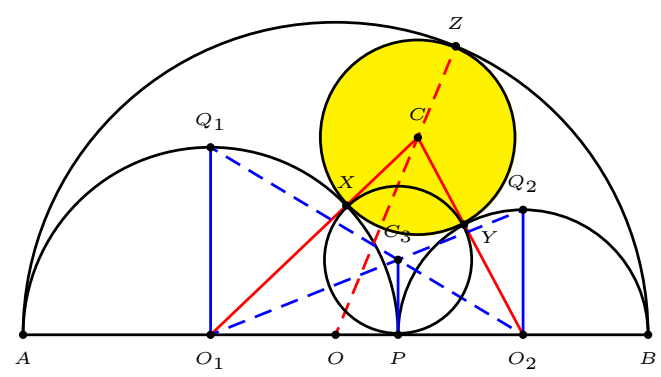


Second construction

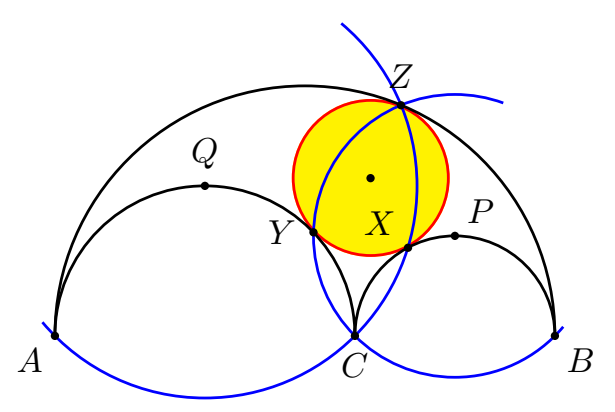

\subsubsection{Woo's three constructions}
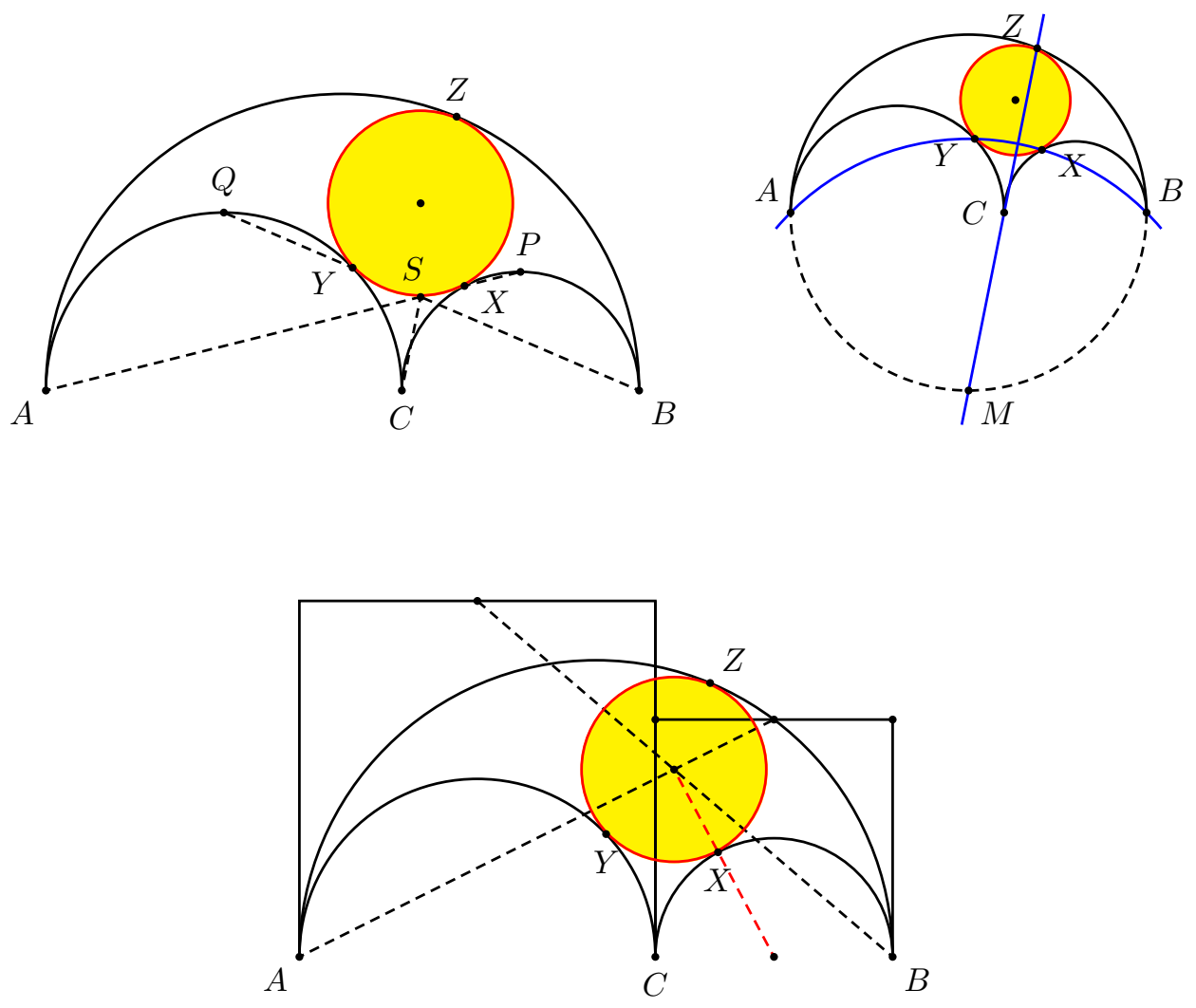


\subsection{More Archimedean circles}

Let $U V$ be the external common tangent of the semicircles $O_{1}(a)$ and $\mathrm{O}_{2}(b)$, which extends to a chord $H K$ of the semicircle $O(a+b)$. Let $C_{4}$ be the intersection of $\mathrm{O}_{1} \mathrm{~V}$ and $\mathrm{O}_{2} U$. Since

$$
O_{1} U=a, \quad O_{2} V=b, \quad \text { and } O_{1} P: P O_{2}=a: b,
$$

$C_{4} P=\frac{a b}{a+b}=t$. This means that the circle $C_{4}(t)$ passes through $P$ and touches the common tangent $H K$ of the semicircles at $N$.

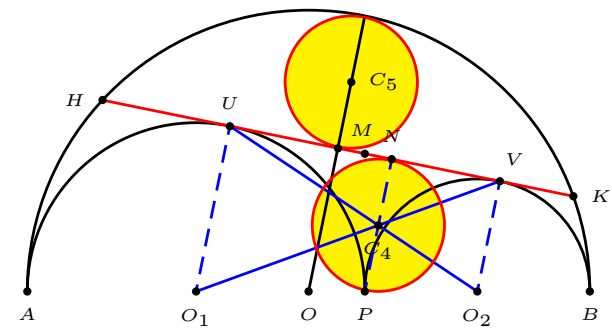

Let $M$ be the midpoint of the chord $H K$. Since $O$ and $P$ are symmetric (isotomic conjugates) with respect to $\mathrm{O}_{1} \mathrm{O}_{2}$,

$$
O M+P N=O_{1} U+O_{2} V=a+b .
$$

it follows that $(a+b)-Q M=P N=2 t$. From this, the circle tangent to $H K$ and the minor arc $H K$ of $O(a+b)$ has radius $t$. This circle touches the minor arc at the point $Q$.

Theorem 20.4 (Thomas Schoch). The incircle of the curvilinear triangle bounded by the semicircle $O(a+b)$ and the circles $A(2 a)$ and $B(2 b)$ has radius $t=\frac{a b}{a+b}$.

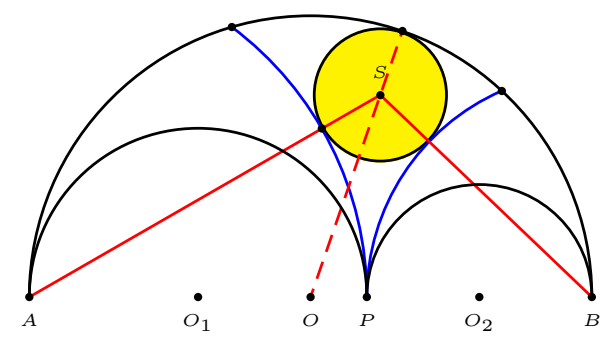

Proof. Denote this circle by $S(x)$. Note that $S O$ is a median of the triangle $\mathrm{SO}_{1} \mathrm{O}_{2}$. By Apollonius theorem,

$$
(2 a+x)^{2}+(2 b+x)^{2}=2\left[(a+b)^{2}+(a+b-x)^{2}\right] .
$$

From this, $x=\frac{a b}{a+b}=t$. 


\section{Exercise}

1. The circles $\left(C_{1}\right)$ and $\left(C_{1}^{\prime}\right)$ are each tangent to the outer semicircle of the shoemaker's knife, and to $O Q_{1}$ at $Q_{1}$; similarly for the circles $\left(C_{2}\right)$ and $\left(C_{2}^{\prime}\right)$. Show that they have equal radii $t=\frac{a b}{a+b}$.

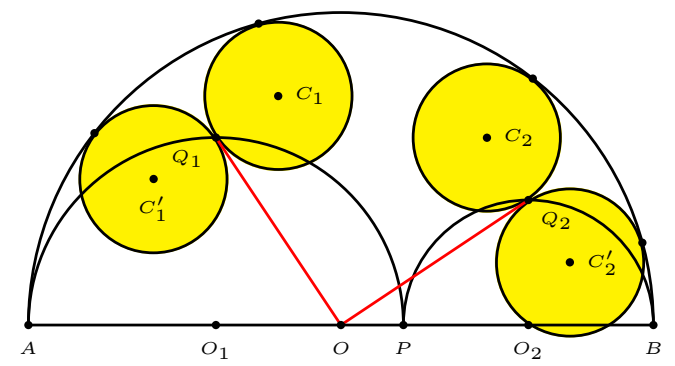

2. We call the semicircle with diameter $O_{1} O_{2}$ the midway semicircle of the shoemaker's knife.

Show that the circle tangent to the line $P Q$ and with center at the intersection of $\left(O_{1}\right)$ and the midway semicircle has radius $t=\frac{a b}{a+b}$.

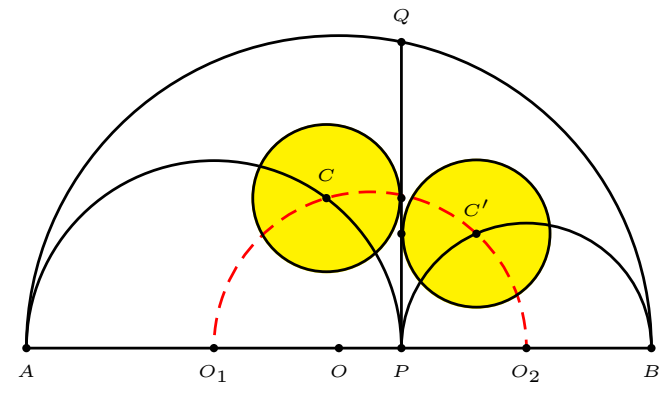

3. Show that the radius of the circle tangent to the midway semicircle, the outer semicircle, and with center on the line $P Q$ has radius $t=\frac{a b}{a+b}$.

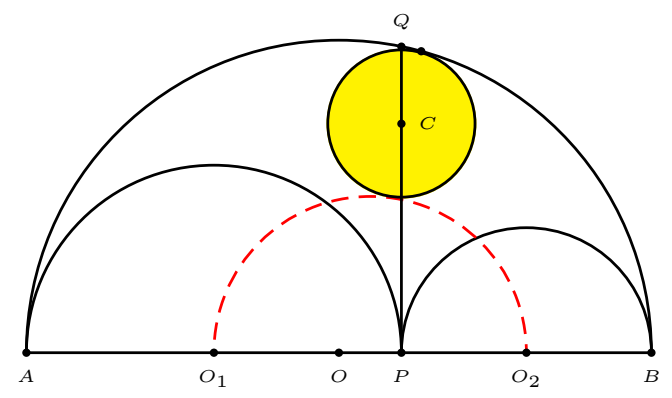




\section{Chapter 21}

\section{Infinitude of prime numbers}

1 Fibonacci and Fermat numbers

2 Somos sequences

3 Fürstenberg's topological proof made easy

Appendix: Euclid's proof

Appendix: The prime numbers below 20,000

Exercise

Project: Prime links

Project: Truncatable primes 


\subsection{Proofs by construction of sequence of relatively prime numbers}

\section{Fibonacci numbers ${ }^{1}$}

Since $\operatorname{gcd}\left(F_{m}, F_{n}\right)=F_{\operatorname{gcd}(m, n)}$, if there are only finitely many primes $p_{1}$, $\ldots, p_{k}$, then the primes in $F_{p_{1}}, \ldots, F_{p_{k}}$ are distinct, and each one of them has only one prime divisors. This contradicts $F_{19}=4181=37 \times 113$.

\section{Fermat numbers}

The Fermat numbers are $F_{n}:=2^{2^{n}}+1$. It is well known that Fermat's conjecture of the primality of $F_{n}$ is wrong. While

$$
F_{0}=3, \quad F_{1}=5, \quad F_{2}=17, \quad F_{3}=257, \quad F_{4}=65537
$$

are all primes, Euler found that

$$
F_{5}=2^{32}+1=4294967297=641 \times 6700417 .
$$

Note that

$$
F_{n}-2=2^{2^{n}}-1=\left(2^{2^{n-1}}+1\right)\left(2^{2^{n-1}}-1\right)=F_{n-1}\left(F_{n-1}-2\right) .
$$

By induction,

$$
F_{n}=F_{n-1} F_{n-2} \cdots F_{1} \cdot F_{0}+2, \quad n \geq 1 .
$$

From this, we see that $F_{n}$ does not contain any factor of $F_{0}, F_{1}, \ldots$, $F_{n-1}$. Hence, the Fermat numbers are pairwise relatively prime. From this, it follows that there are infinitely primes.

\footnotetext{
${ }^{1}$ M. Wunderlich, Another proof of the infinite primes theorem, American Math. Monthly, 72 (1965) 305.
} 


\subsection{Somos sequences}

Define two sequences $\left(a_{n}\right)$ and $\left(b_{n}\right)$ with initial values

$$
a_{0}=a_{1}=1, \quad b_{0}=0, b_{1}=1,
$$

and for $n \geq 2$ recursively by

$$
\begin{aligned}
a_{n} & =a_{n-2} b_{n-1}, \\
b_{n} & =a_{n-1}+a_{n} .
\end{aligned}
$$

Here are the first few terms

\begin{tabular}{c|ccccccccccc}
$n$ & 0 & 1 & 2 & 3 & 4 & 5 & 6 & 7 & 8 & 9 & $\cdots$ \\
\hline$a_{n}$ & 1 & 1 & 1 & 2 & 3 & 10 & 39 & 490 & 20631 & 10349290 & $\cdots$ \\
$b_{n}$ & 0 & 1 & 2 & 3 & 5 & 13 & 49 & 529 & 21121 & 10369921 & $\cdots$
\end{tabular}

Theorem 21.1. The terms of the sequence $\left(b_{n}\right), n \geq 1$ are relatively prime.

Proof. If we calculate the first few terms

$$
\begin{aligned}
& a_{2}=a_{0} b_{1}=b_{1}, \\
& a_{3}=a_{1} b_{2}=b_{2}, \\
& a_{4}=a_{2} b_{3}=b_{1} b_{3}, \\
& a_{5}=a_{3} b_{4}=b_{2} b_{4}, \\
& a_{6}=a_{4} b_{5}=b_{1} b_{3} b_{5}, \\
& a_{7}=a_{5} b_{6}=b_{2} b_{4} b_{6},
\end{aligned}
$$

we see a pattern, namely,

$$
\begin{aligned}
a_{2 n} & =b_{1} b_{3} \cdots b_{2 n-1}, \\
a_{2 n+1} & =b_{2} b_{4} \cdots b_{2 n} .
\end{aligned}
$$

Suppose, inductively, that $b_{1}, b_{2}, \ldots, b_{2 n-1}$ are relatively prime. Then,

$$
b_{2 n}=a_{2 n-1}+a_{2 n}=b_{2} b_{4} \cdots b_{2 n-2}+b_{1} b_{3} \cdots b_{2 n-1}
$$

does not contain any divisor of $b_{1}, b_{2}, \ldots, b_{2 n-1}$; nor does

$$
b_{2 n+1}=a_{2 n}+a_{2 n+1}=b_{1} b_{3} \cdots b_{2 n-1}+b_{2} b_{4} \cdots b_{2 n}
$$

contain any divisor of $b_{1}, b_{2}, \ldots, b_{2 n}$. It follows by induction that no two of the terms $b_{1}, b_{2}, \ldots, b_{n}, \ldots$ contain a common divisor. 


\title{
21.3 Fürstenberg's topological proof made easy
}

There is a famous proof of the infinitude of primes using topology. It can be found in many books. Apart from an introductory sentence, here is the entire article of [Fürstenberg]:

\begin{abstract}
We introduce a topology into the space of integers $S$, by using the arithmetic progressions (from $-\infty$ to $+\infty$ ) as a basis. It is not difficult to verify that this actually yields a topological space. In fact, under this topology, $S$ may be shown to be normal and hence metrizable. Each arithmetic progression is closed as well as open, since its complement is the union of other arithmetic progressions (having the same difference). As a result, the union of any finite number of arithmetic progression is closed. Consider now the set $A=\bigcup A_{p}$, where $A_{p}$ consists of all multiples of $p$, and $p$ runs through the set of primes $\geq 2$. The only numbers not belonging to $A$ are -1 and 1 , and since the set $\{-1,1\}$ is clearly not an open set, $A$ cannot be closed. Hence $A$ is not a finite union of closed sets which proves that there are an infinity of primes.
\end{abstract}

The most recent issue of Mathematics Magazine ${ }^{2}$ contains a paraphrase of this proof, avoiding the language of topology.

Let $\mathbb{Z}$ be the set of integers. We say that a subset $A \subset Z$ is periodic if there is an integer $k$ such that for every integer $n \in \mathbb{Z}, n \in A$ if and only if $n+k \in A$. In other words, a period set is a (finite) union of doubly infinite arithmetic progressions of the same common difference. The following are clear.

1. If $A$ is periodic, then so is its complement $\mathbb{Z} \backslash A$.

2. If $A$ and $B$ are periodic sets, so is their union, the period of the union being the $1 \mathrm{~cm}$ of the periods of the sets. For example, if $A_{3}:=\{3 n: n \in Z\}$ and $A_{5}:=\{5 n: n \in Z\}$, then $A_{3} \cup A_{5}$ is the union of the arithmetic progressions of common difference 15 , containing the terms $0,3,5,6,9,10$.

3. (2) extends to finite unions.

For each prime number $p$, let $A_{p}:=\{n p: n \in \mathbb{Z}\}$ consists of the multiples of $p$. This is clearly periodic. Suppose there are only finitely many primes. Then, the (finite) union $A:=\bigcup_{p \text { prime }} A_{p}$ is periodic, and so is its complement. This complement is clearly the set $\{-1,1\}$, which, being finite, cannot be periodic. This contradiction shows that there are indeed infinitely many primes.

\footnotetext{
${ }^{2}$ Math. Mag., 76 (2003) number 3.
} 


\section{Appendix: Euclid's proof ${ }^{3}$}

The prime numbers or primes are the numbers

$$
2,3,5,7,11,13,17,19,23,29, \ldots
$$

which cannot be resolved into smaller factors. ... We have to prove that there are infinitely many primes, i.e., that the series $(A)$ never comes to an end.

Let us suppose that it does, and that

$$
2,3,5, \ldots, P
$$

is the complete series (so that $P$ is the largest prime); and let us, on this hypothesis, consider the number $Q$ defined by the formula

$$
Q=(2 \cdot 3 \cdot 5 \cdots P)+1 \text {. }
$$

It is plain that $Q$ is not divisible by any of $2,3,5, \ldots, P$; for it leaves the remainder 1 when divided by any one of these numbers. But, if not itself prime, it is divisible by some prime, and therefore there is a prime (which may be $Q$ itself) greater than any of them. This contradicts our hypothesis, that there is no prime greater than $P$; and therefore this hypothesis is false.

The proof is by reductio ad absurdum, and reductio ad absurdum, which Euclid loved so much, is one of a mathematician's finest weapon. It is a far finer gambit than any chess gambit: a chess player may offer the sacrifice of a pawn or even a prize, but a mathematician offers the game.

\footnotetext{
${ }^{3}$ G. H. Hardy’s paraphrase. [Hardy, pp.95-96].
} 


\section{Appendix: The prime numbers below 20000}

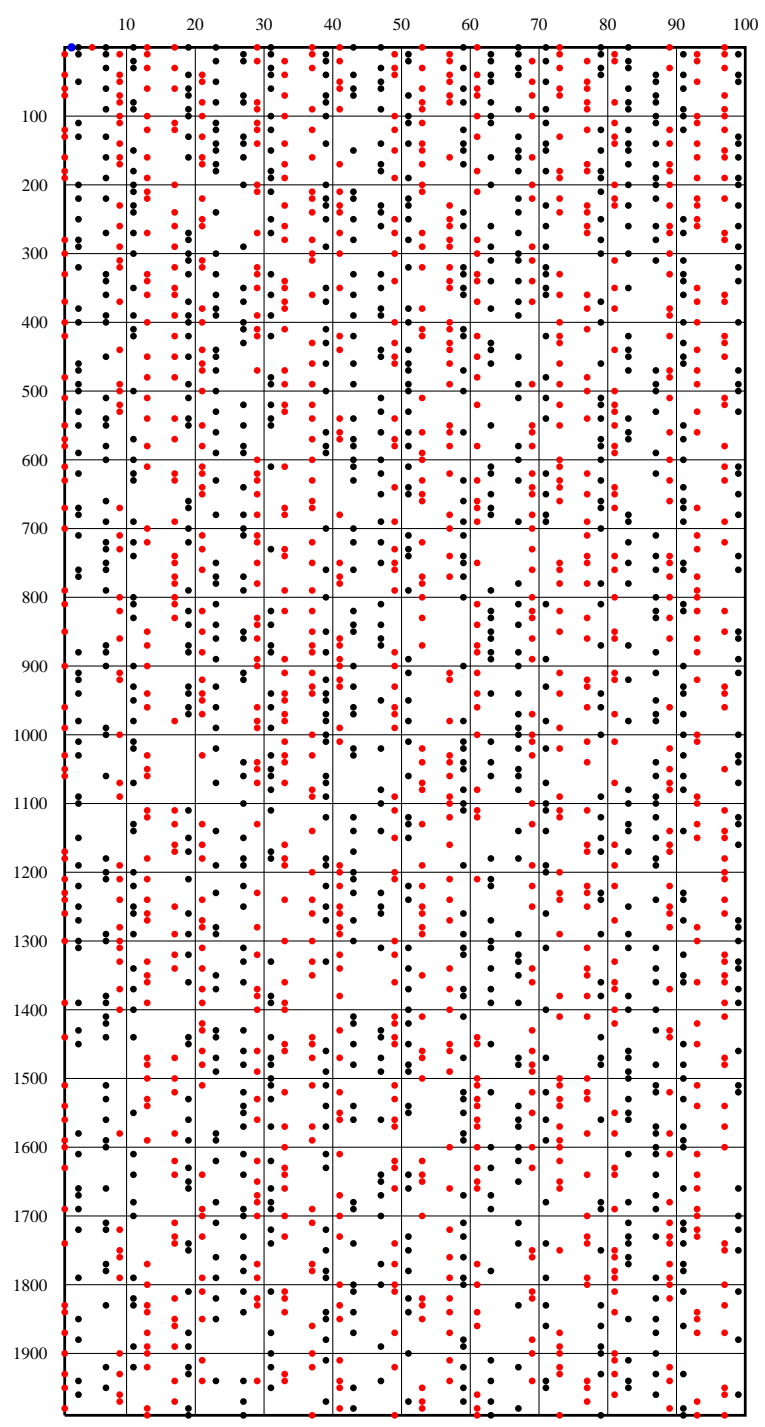




\section{Exercise}

1. Let $p_{n}$ denote the $n$-th prime number. Find the smallest value of $n$ for which $p_{1} p_{2} \cdots p_{n}+1$ is not a prime number.

2. Find the smallest value of $n$ for which $p_{1} p_{2} \cdots p_{n}-1$ is not a prime number.

3. Find the smallest value of $n>3$ for which $n !+1$ is a prime number.

4. Find the smallest value of $n>7$ for which $n !-1$ is a prime number.

5. Find a shortest sequence of prime numbers $p_{1}<p_{2}<\cdots<p_{n}$ satisfying the following conditions.

(i) $p_{1}=2$,

(ii) $p_{k+1}<2 p_{k}$ for $k=1, \ldots, n-1$, (iii) $p_{n}>10000$. 


\section{Project: Prime links}

A prime link of length $n$ is a permutation of $1,2, \ldots n$ beginning with 1 and ending with $n$ such that the sum of each pair of adjacent terms is prime. This was proposed and solved by Morris Wald [157]. For $n \leq 6$, the link is unique. For $n=7$ there are two links: 1, 4, 3, 2, 5, 6, 7 and and $1,6,5,2,3,4,7$. Wald suggested working backwards. Start with $n$ and precede it with the greatest remaining member of the set whose sum with $n$ is a prime, and repeat in like fashion. Here are the first 10 links:

1.

1,2 .

$1,2,3$.

$1,2,3,4$.

$1,4,3,2,5$.

$1,4,3,2,5,6$.

$1,4,3,2,5,6,7$.

$1,2,3,4,7,6,5,8$.

$1,2,3,4,7,6,5,8,9$.

$1,2,3,4,7,6,5,8,9,10$.

Continue with larger values of $n$. 


\section{Project: Tuncatable primes}

Consider the prime number 73939133 . The numbers obtained by truncations from the right are all primes:

$$
\text { 73939133, 7393913, 739391, 73939, 7393, 739, 73, } 7 .
$$

More generally, we call a number $N$ a right-truncatable prime if every number obtained from truncating $N$ from the right is a prime or 1 .

A complete list of right-truncatable primes can be found in [Waltrom and Berg].

Write a computer program to find all right-truncatable primes.

Similarly, define left-truncatable primes, and bi-truncatable primes as those which are both left- and right-truncatable primes.

$\begin{array}{rrrr}53 & 3797 & 73331 & 7393931 \\ & 7331 & 373393 & 7393933 \\ 317 & 23333 & 593993 & 23399339 \\ 599 & 23339 & 719333 & 29399999 \\ 797 & 31193 & 739397 & 37337999 \\ 2393 & 31379 & 739399 & 59393339 \\ 3793 & 37397 & 2399333 & 73939133\end{array}$

Here are all the bi-truncatable primes:

$\begin{array}{lllll}1 & 2 & 3 & 5 & 7 \\ 11 & 13 & 17 & 23 & 31 \\ 37 & 53 & 71 & 73 & 113 \\ 131 & 137 & 173 & 311 & 313 \\ 317 & 373 & 1373 & 3137 & \end{array}$




\section{Chapter 22}

\section{Strings of prime numbers}

1 The prime number spiral

2 The prime number spiral beginning with 17

3 The prime number spiral beginning with 41

Appendix: The number spiral

Appendix: Long chains of primes

Appendix: Consecutive primes with consecutive prime digital sums

Projects 


\subsection{The prime number spiral}

The first 1000 prime numbers arranged in a spiral.

- = prime of the form $4 n+1$;

•= prime of the form $4 n+3$.

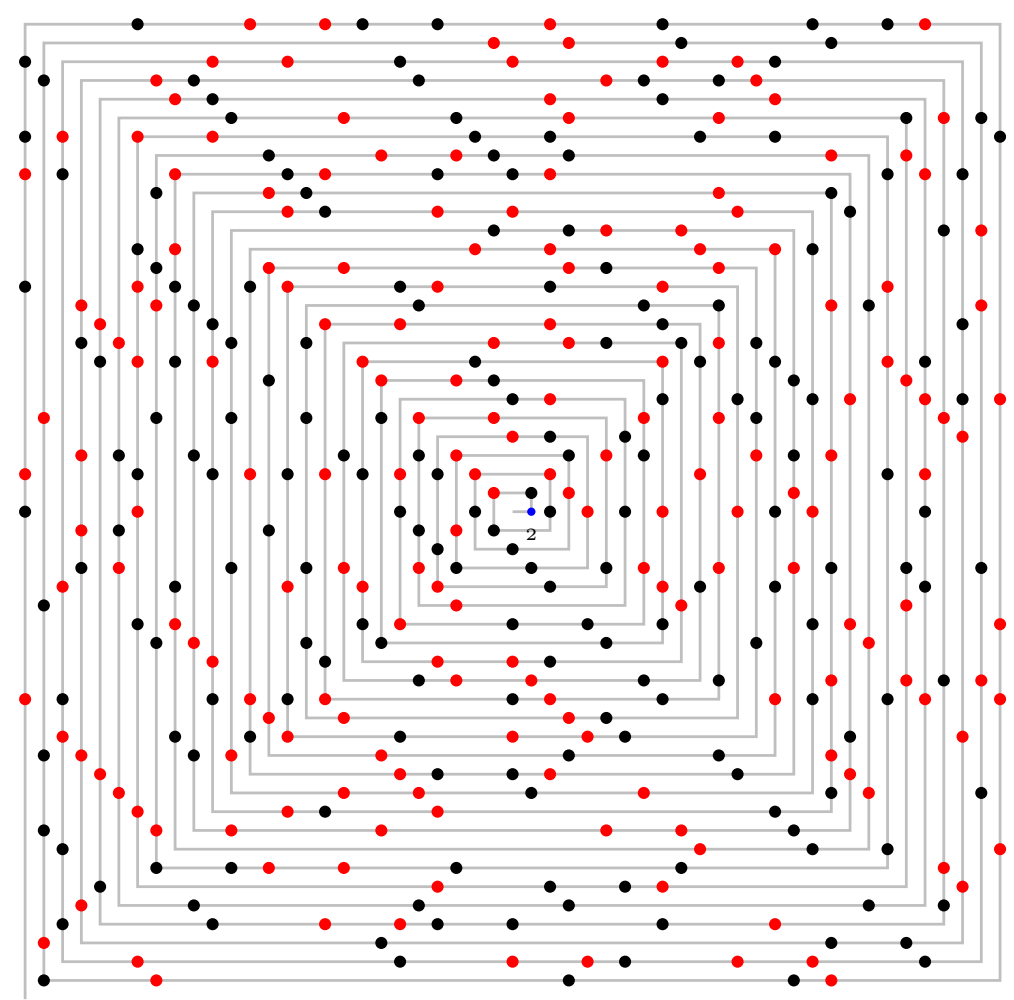




\subsection{The prime number spiral beginning with 17}

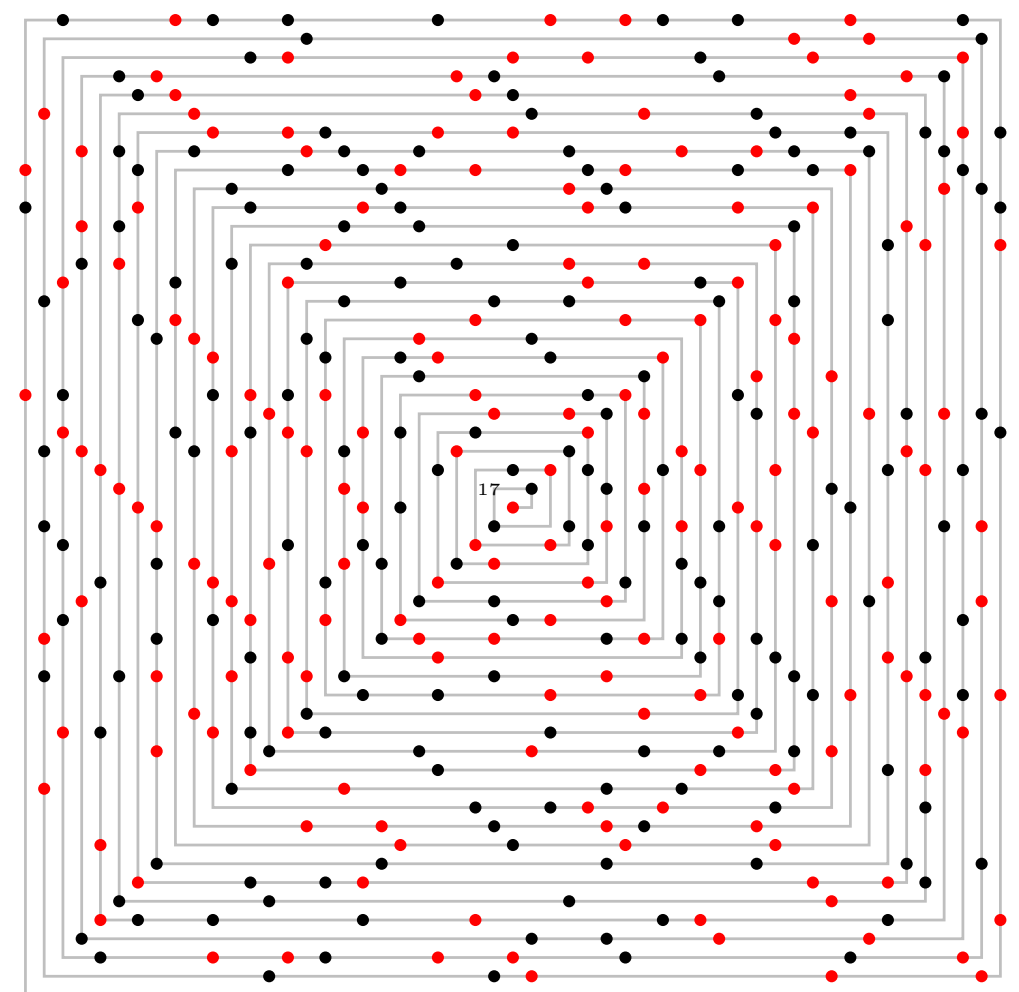

The numbers on the 45 degree line are $n^{2}+n+17$.

Let $f(n)=n^{2}+n+17$. The numbers $f(0), f(1), \ldots f(15)$ are all prime.

\begin{tabular}{cccccccc}
$n$ & $f(n)$ & $n$ & $f(n)$ & $n$ & $f(n)$ & $n$ & $f(n)$ \\
\hline 0 & 17 & 1 & 19 & 2 & 23 & 3 & 29 \\
4 & 37 & 5 & 47 & 6 & 59 & 7 & 73 \\
8 & 89 & 9 & 107 & 10 & 127 & 11 & 149 \\
12 & 173 & 13 & 199 & 14 & 227 & 15 & 257
\end{tabular}




\subsection{The prime number spiral beginning with 41}

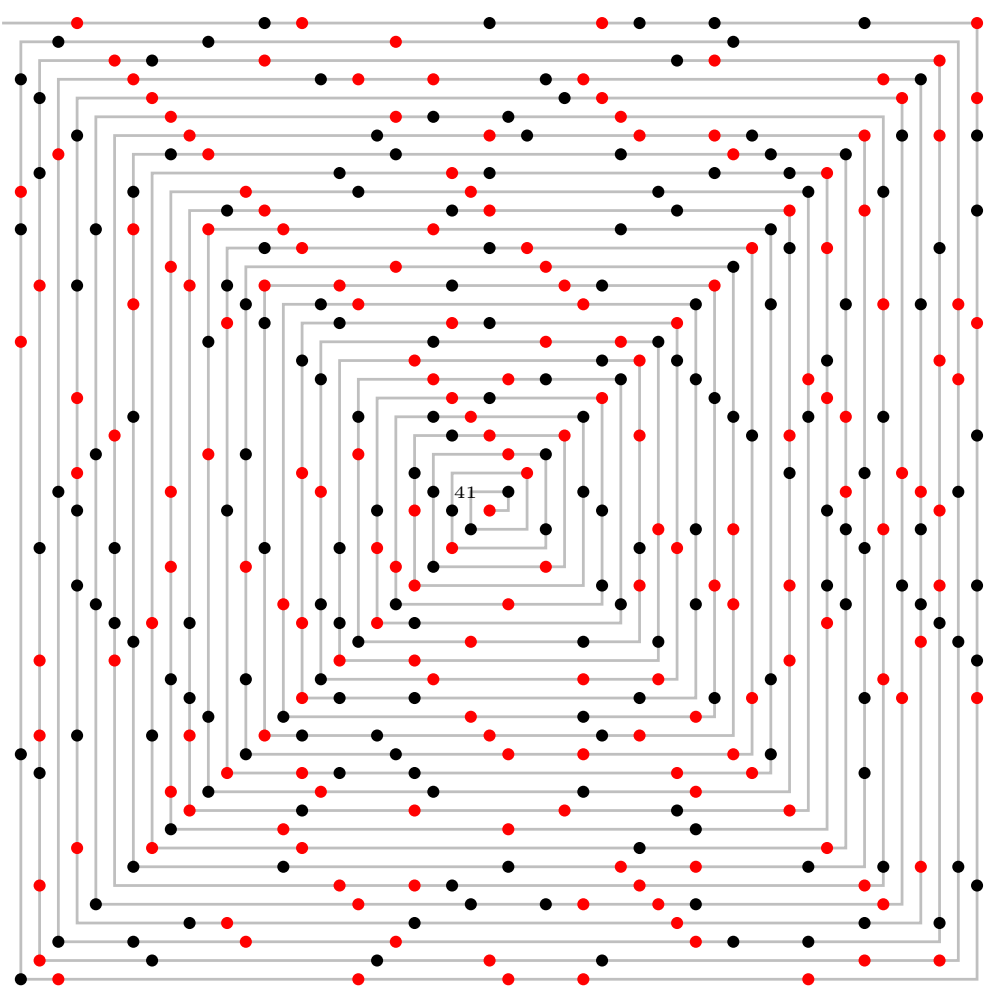

The numbers on the 45 degree line are $f(n)=n^{2}+n+41$. $f(n)=n^{2}+n+41$ is prime for $0 \leq n \leq 39$.

\begin{tabular}{cccccccccc}
$n$ & $f(n)$ & $n$ & $f(n)$ & $n$ & $f(n)$ & $n$ & $f(n)$ & $n$ & $f(n)$ \\
\hline 0 & 41 & 1 & 43 & 2 & 47 & 3 & 53 & 4 & 61 \\
5 & 71 & 6 & 83 & 7 & 97 & 8 & 113 & 9 & 131 \\
10 & 151 & 11 & 173 & 12 & 197 & 13 & 223 & 14 & 251 \\
15 & 281 & 16 & 313 & 17 & 347 & 18 & 383 & 19 & 421 \\
20 & 461 & 21 & 503 & 22 & 547 & 23 & 593 & 24 & 641 \\
25 & 691 & 26 & 743 & 27 & 797 & 28 & 853 & 29 & 911 \\
30 & 971 & 31 & 1033 & 32 & 1097 & 33 & 1163 & 34 & 1231 \\
35 & 1301 & 36 & 1373 & 37 & 1447 & 38 & 1523 & 39 & 1601
\end{tabular}


Prime number spiral beginning with 41: A closer look

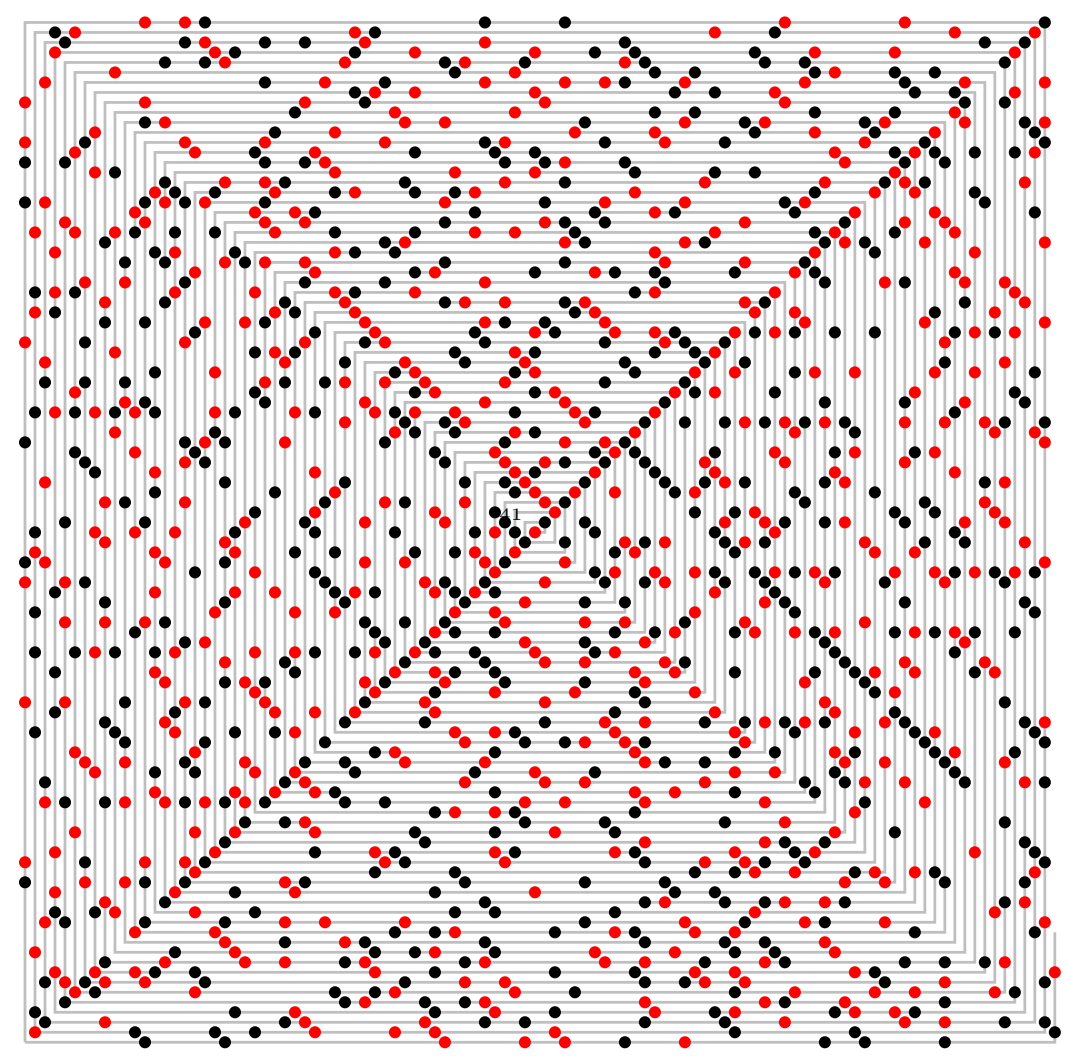




\section{Appendix: The number spiral}

Beginning with the origin, we trace out a spiral clockwise through the lattice points. Along with this, we label the lattice points $0,1,2, \ldots$ consecutively.

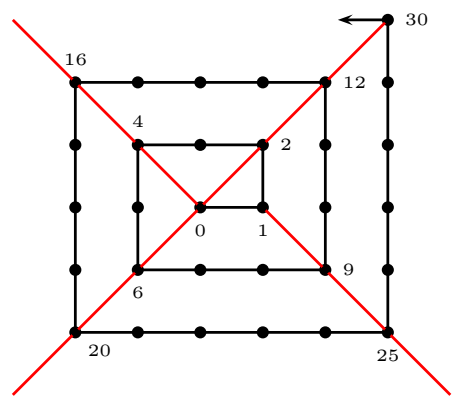

Given a positive integer $N$, let $(2 m-1)^{2}$ be the largest odd square $\leq N$, and write

$$
N=(2 m-1)^{2}+q, \quad 0 \leq q<8 m .
$$

Then the number $N$ appears at the lattice point

$$
\begin{cases}(m,-m+q+1) & \text { if } q \leq 2 m-1 \\ (3 m-q-1, m) & \text { if } 2 m-1 \leq q \leq 4 m-1 \\ (-m, 5 m-q-1) & \text { if } 4 m-1 \leq q \leq 6 m-1 \\ (-7 m+q+1,-m) & \text { if } 6 m-1 \leq q \leq 8 m-1\end{cases}
$$

Denote by $f(m, n)$ the number at the lattice point $(m, n)$.

It is clear that along the 45-degree line, $f(n, n)=2 n(2 n-1)$. Also,

$$
f(-n, n)=4 n^{2} \quad \text { if } n \geq 0,
$$

and

$$
f(n,-(n-1))=(2 n-1)^{2} \quad \text { if } n>0 .
$$

More generally,

$$
f(m, n)= \begin{cases}4 m^{2}-3 m+n & \text { if } m>|n|, \\ 4 m^{2}-m-n & \text { if }-m=|m|>|n|, \\ 4 n^{2}-n-m & \text { if } n>|m|, \\ 4 n^{2}-3 n+m & \text { if }-n=|n|>|m| .\end{cases}
$$




\section{Exercise}

1. Label the vertices of a cube with the numbers 1 through 8 in such a way that the sum of the endpoints of each edge is prime.

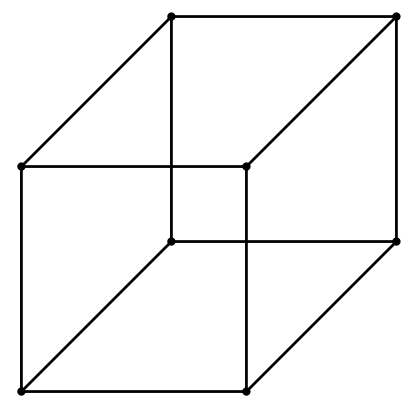




\section{Appendix: Long chains of primes}

Beiler [p.220] also considers the cubic function $f(n)=n^{3}+n^{2}+17$, and noted that for $n=-14,-13, \cdots, 10$, the string of 25 values are all primes. This is true only when we take -1 as a prime, since $f(-3)=$ -1 . Even if we break the string into two, we still get two long chains of primes:

$f(-14), f(-13), \ldots, f(-4)$ a chain of 11 primes.

$f(-2), f(-1), f(0), \ldots, f(10)$. But $f(0)=f(-1)=17$, we only have 12 distinct primes.

Beyond these, the longest strings of primes have 6 members. The first of these begin with $n=717$.

Note that on the negative side, there is a string of 10 consecutive primes from -183 to -174 . Replacing $n$ by $-n$ we consider $n^{3}-n-17$ for $n=174, \ldots, 183$ :

\begin{tabular}{ccl}
$n$ & $n^{3}+n^{2}+17$ & factorization \\
\hline 173 & 5147771 & $683 \times 7537$ \\
174 & 5237731 & prime \\
175 & 5328733 & prime \\
176 & 5420783 & prime \\
177 & 5513887 & prime \\
178 & 5608051 & prime \\
179 & 5703281 & prime \\
180 & 5799583 & prime \\
181 & 5896963 & prime \\
182 & 5995427 & prime \\
183 & 6094981 & prime \\
184 & 6195631 & $13 \times 476587$ \\
185 & 6297383 & prime
\end{tabular}

Higgins: 40 primes from $g(x)=9 x^{2}-231 x+1523, x=0, \ldots, 39$. or $h(x)=9 x^{2}-471 x+6203$ give the same primes in reverse order. 


\section{Appendix: Consecutive primes with consecutive prime digital sums}

Charles Twigg asked, in Crux Math., Problem 228, for four consecutive primes having digital sums that, in some order, are consecutive primes. And then five.

The beginning of the prime number sequence provides an easy answer: just consider the primes $2,3,5,7$, or 3, 5, 7, 11. Beyond these, the first quadruple is $191,193,197$, and 199 , with digit sums $11,13,17,19$.

The five consecutive primes $311,313,317,331,337$ all have prime digital sums, though these are the same for 313 and 331.

The first sequence of five consecutive primes who digital sums form another sequence of 5 consecutive primes is

$$
(1291,13),(1297,19),(1301,5),(1303,7),(1307,11) .
$$

Twigg listed such quadruples and quintuples up to primes around 5 million (about 350000 primes). But within this range he had overlooked the better records

$$
(102251,11),(102253,13),(102259,19),(102293,17),(102299,23),(102301,7)
$$

and

$\begin{array}{llll}3511973,29 & 3511993,31 & 3511999,37 & 3512011,13 \\ 3512051,17 & 3512053,19 & 3512057,23 & \end{array}$

A little bit beyond these we find the best record for eight up to the first 1 million primes:

$\begin{array}{llll}5919931,37 & 5919937,43 & 5919959,47 & 5919971,41 \\ 5920003,19 & 5920043,23 & 5920049,29 & 5920069,31\end{array}$

Within the same range, there are also 15 consecutive primes whose digital sums are primes, though only with 5 different values:

$\begin{array}{lllll}2442113,17 & 2442133,19 & 2442151,19 & 2442173,23 & 2442179,29 \\ 2442191,23 & 2442197,29 & 2442199,31 & 2442227,23 & 2442263,23 \\ 2442287,29 & 2442289,31 & 2442311,17 & 2442353,23 & 2442359,29\end{array}$

Another "long" chain of 9 consecutive primes with 5 different consecutive prime digital sums can be found among "small" primes:

$\begin{array}{lllll}14293,19 & 14303,11 & 14321,11 & 14323,13 & 14327,17 \\ 14341,13 & 14347,19 & 14369,23 & 14387,23 & \end{array}$




\section{Project}

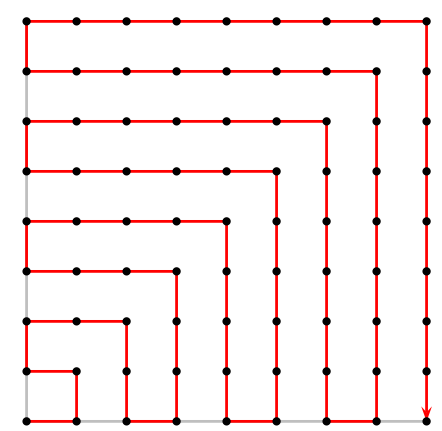

Find the coordinates of the $n$-th point of the path, beginning with the origin.

What is the position of the lattice point $(a, b)$ in the sequence? 


\section{Chapter 23}

\section{Strings of composites}

1 Strings of consecutive composite numbers

2 Strings of consecutive composite values of $n^{2}+1$

3 Strings of consecutive composite values of $n^{2}+n+41$

Appendix: 50 consecutive composite values of $x^{2}+x+41$

Project 


\subsection{Strings of consecutive composite numbers}

It is well known that there are strings of consecutive composite numbers

of arbitrary lengths. For example, the $n$ numbers

$$
(n+1) !+2,(n+1) !+3, \cdots,(n+1) !+(n+1)
$$

are all composites. These numbers are, however, very large.

In the table below, we give the first string of $n$ consecutive composite numbers.

\begin{tabular}{cl}
$n$ & first string of $n$ composite numbers \\
\hline 3 & $8 \cdots 10$ \\
5 & $24 \cdots 28$ \\
7 & $90 \cdots 96$ \\
13 & $114 \cdots 126$ \\
17 & $524 \cdots 540$ \\
19 & $888 \cdots 906$ \\
21 & $1130 \cdots 1150$ \\
33 & $1328 \cdots 1360$ \\
35 & $9552 \cdots 9586$ \\
43 & $15684 \cdots 15726$ \\
51 & $19610 \cdots 19660$ \\
71 & $31398 \cdots 31468$ \\
85 & $155922 \cdots 156006$ \\
95 & $360654 \cdots 360748$ \\
111 & $370262 \cdots 370372$ \\
113 & $492114 \cdots 492226$ \\
117 & $1349534 \cdots 1349650$ \\
131 & $1357202 \cdots 1357332$ \\
147 & $2010734 \cdots 2010880$ \\
153 & $4652354 \cdots 4652506$ \\
179 & $17051708 \cdots 17051886$ \\
209 & $20831324 \cdots 20831532$
\end{tabular}

The first string of 100 consecutive composite numbers begins with 370262. ${ }^{1}$ These are significantly less than 101 !.

\footnotetext{
${ }^{1}$ It actually extends to 370372 , with 111 composite numbers. The string beginning with 396734 just misses by 1 ; it gives 99 consecutive composites.
} 


\subsection{Strings of consecutive composite values of $n^{2}+1$}

\begin{tabular}{cl}
$m$ & $n \cdots n+m-1$ \\
\hline 3 & $7 \cdots 9$ \\
9 & $27 \cdots 35$ \\
13 & $41 \cdots 53$ \\
15 & $95 \cdots 109$ \\
19 & $185 \cdots 203$ \\
33 & $351 \cdots 383$ \\
39 & $497 \cdots 535$ \\
45 & $3391 \cdots 3435$ \\
87 & $3537 \cdots 3623$ \\
99 & $45371 \cdots 45469$ \\
111 & $82735 \cdots 82845$ \\
129 & $99065 \cdots 99193$ \\
151 & $357165 \cdots 357315$ \\
211 & $840905 \cdots 841115$
\end{tabular}




\subsection{Consecutive composite values of $x^{2}+x+41$}

Problem 142 of Crux Mathematicorum asks for 40 consecutive positive integer values of $x$ for which $f(x)=x^{2}+x+41$ are all composites. Several solutions were published. Unfortunately these numbers were quite large, being constructed by a method similar to the one above. For example, here is one. Since for $n=0, \ldots, 39, f(n) \leq f(39)=1601$, if we set $x_{n}=1601 !+n$, then

$$
f\left(x_{n}\right)=x_{n}^{2}+x_{n}+41=(1601 !) \cdot(1601 !+2 b+1)+f(n)
$$

is a multiple of $f(n)$ which is greater than $f(n)$. These numbers are therefore composite. These numbers are quite large since 1601! has 4437 digits. The Crux editor wrote that "[i]t would be interesting if some computer nut were to make a search and discover the smallest set of 40 consecutive integers $x$ for which $f(x)$ is composite".

A near miss is 176955 . The string of 38 consecutive numbers beginning with this all give composite $f(x)$. H. L. Nelson, then (and now) editor of Journal of Recreational Mathematics, found this smallest string, with factorization of the corresponding $f(x)$. It begins with 1081296 .

There are longer strings. ${ }^{2}$ Up to 5,000,000, the longest string of composites has 50 numbers. There are three such strings, beginning with 2561526, 3033715, and 3100535 respectively. See Appendix 2 for the first of these strings.

How about long strings of primes? They are relatively few. The only strings of $\geq 10$ consecutive primes begin with 66, 191, 219, 534, and 179856, and no more up to 5,000,000. Each of these strings contains 10 primes, except the one beginning with 219 , which contains 13 primes.

\footnotetext{
${ }^{2}$ For example, beginning with 1204431 , we have a string of 45 composites.
} 
Appendix: 50 consecutive composite values of $x^{2}+x+41$

\begin{tabular}{|c|c|c|}
\hline$x$ & $x^{2}+x+41$ & Factorization \\
\hline 2561525 & 6561412887191 & prime \\
\hline 2561526 & 6561418010243 & $1693357 \times 3874799$ \\
\hline 2561527 & 6561423133297 & $1097 \times 5981242601$ \\
\hline 2561528 & 6561428256353 & $167 \times 971 \times 40463429$ \\
\hline 2561529 & 6561433379411 & $499 \times 13149165089$ \\
\hline 2561530 & 6561438502471 & $167 \times 797 \times 853 \times 57793$ \\
\hline 2561531 & 6561443625533 & $773 \times 8488284121$ \\
\hline 2561532 & 6561448748597 & $71 \times 133261 \times 693487$ \\
\hline 2561533 & 6561453871663 & $379 \times 7591 \times 2280667$ \\
\hline 2561534 & 6561458994731 & $1512947 \times 4336873$ \\
\hline 2561535 & 6561464117801 & $39233 \times 167243497$ \\
\hline 2561536 & 6561469240873 & $347 \times 2339 \times 8084281$ \\
\hline 2561537 & 6561474363947 & $367 \times 17878676741$ \\
\hline 2561538 & 6561479487023 & $3049 \times 2152010327$ \\
\hline 2561539 & 6561484610101 & $53 \times 83 \times 661 \times 2256559$ \\
\hline 2561540 & 6561489733181 & $3947 \times 1662399223$ \\
\hline 2561541 & 6561494856263 & $10501 \times 624844763$ \\
\hline 2561542 & 6561499979347 & $3557 \times 1844672471$ \\
\hline 2561543 & 6561505102433 & $71 \times 92415564823$ \\
\hline 2561544 & 6561510225521 & $47 \times 139606600543$ \\
\hline 2561545 & 6561515348611 & $722317 \times 9083983$ \\
\hline 2561546 & 6561520471703 & $53 \times 4973 \times 24894887$ \\
\hline 2561547 & 6561525594797 & $2927 \times 2241723811$ \\
\hline 2561548 & 6561530717893 & $419 \times 15659977847$ \\
\hline 2561549 & 6561535840991 & $47^{2} \times 2970364799$ \\
\hline 2561550 & 6561540964091 & $2003 \times 3275856697$ \\
\hline 2561551 & 6561546087193 & $43 \times 919 \times 1039 \times 159811$ \\
\hline 2561552 & 6561551210297 & $83 \times 12577 \times 6285667$ \\
\hline 2561553 & 6561556333403 & $151 \times 43454015453$ \\
\hline 2561554 & 6561561456511 & $43 \times 152594452477$ \\
\hline 2561555 & 6561566579621 & $653 \times 10048340857$ \\
\hline 2561556 & 6561571702733 & $41 \times 160038334213$ \\
\hline 2561557 & 6561576825847 & $41 \times 160038459167$ \\
\hline 2561558 & 6561581948963 & $2053 \times 3196094471$ \\
\hline 2561559 & 6561587072081 & $9049 \times 725117369$ \\
\hline 2561560 & 6561592195201 & $1601 \times 8933 \times 458797$ \\
\hline 2561561 & 6561597318323 & $1994669 \times 3289567$ \\
\hline 2561562 & 6561602441447 & $691 \times 23689 \times 400853$ \\
\hline 2561563 & 6561607564573 & $4111 \times 1596109843$ \\
\hline 2561564 & 6561612687701 & $131 \times 419 \times 1259 \times 94951$ \\
\hline 2561565 & 6561617810831 & $238363 \times 27527837$ \\
\hline 2561566 & 6561622933963 & $4783 \times 1371863461$ \\
\hline 2561567 & 6561628057097 & $2039 \times 3218061823$ \\
\hline 2561568 & 6561633180233 & $61 \times 97 \times 1108945949$ \\
\hline 2561569 & 6561638303371 & $694367 \times 9449813$ \\
\hline 2561570 & 6561643426511 & $5417 \times 6529 \times 185527$ \\
\hline 2561571 & 6561648549653 & $347 \times 18909649999$ \\
\hline 2561572 & 6561653672797 & $4933 \times 1330154809$ \\
\hline 2561573 & 6561658795943 & $5839 \times 1123764137$ \\
\hline 2561574 & 6561663919091 & $151 \times 397 \times 109457753$ \\
\hline 2561575 & 6561669042241 & $313 \times 1999 \times 10487143$ \\
\hline 2561576 & 6561674165393 & prime \\
\hline
\end{tabular}


Project: Strings of consecutive composite values of $n^{2}+n+1$

Find the first strings of consecutive composite values of $n^{2}+n+1$.

$$
\begin{array}{ll}
m & n \cdots n+m-1 \\
\hline 3 & 9 \cdots 11 \\
5 & 28 \cdots 32
\end{array}
$$

\section{Project}

The first string of 99 consecutive composite values of $n^{2}+1$ begins with $n=45371$. The string with more than 100 composite values of $n^{2}+1$ has 111 members beginning with 82735 . What is the longest of such a string you can find? 


\section{Chapter 24}

\section{Perfect numbers}

1 Perfect numbers

2 Charles Twigg on the first 10 perfect numbers

3 Abundant and deficient numbers

Appendix: Mersenne primes

Appendix: Three important number theoretic functions

Exercise 


\subsection{Perfect numbers}

A number is perfect is the sum of its proper divisors (including 1) is equal to the number itself.

Theorem 24.1 (Euclid). If $1+2+2^{2}+\cdots+2^{k-1}=2^{k}-1$ is a prime number, then $2^{k-1}\left(2^{k}-1\right)$ is a perfect number.

Note: $2^{k}-1$ is usually called the $k$-th Mersenne number and denoted by $M_{k}$. If $M_{k}$ is prime, then $k$ must be prime.

Theorem 24.2 (Euler). Every even perfect number is of the form given by Euclid.

\section{Open problem}

Does there exist an odd perfect number?

Theorem-joke 24.1 (Hendrik Lenstra). Perfect squares do not exist. ${ }^{1}$

Proof. Suppose $n$ is a perfect square. Look at the odd divisors of $n$. They all divide the largest of them, which is itself a square, say $d^{2}$. This shows that the odd divisors of $n$ come in pairs $a, b$ where $a \cdot b=d^{2}$. Only $d$ is paired to itself. Therefore the number of odd divisors of $n$ is also odd. In particular, it is not $2 n$. Hence $n$ is not perfect, a contradiction: perfect squares don't exist.

\footnotetext{
${ }^{1}$ Math. Intelligencer, 13 (1991) 40.
} 


\subsection{Charles Twigg on the first 10 perfect numbers}

There are only 39 known Mersenne primes, and therefore 39 known perfect numbers. See Appendix. Let $P_{n}$ be the $n$-th perfect number.

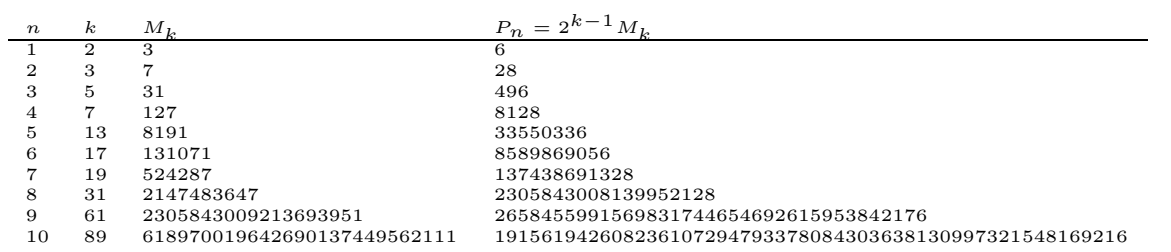

- $P_{1}$ is the difference of the digits of $P_{2}$. In $P_{2}$, the units digit is the cube of the of tens digit.

- $P_{3}$ and $P_{4}$ are the first two perfect numbers prefaced by squares. The first two digits of $P_{3}$ are consecutive squares. The first and last digits of $P_{4}$ are like cubes. The sums of the digits of $P_{3}$ and $P_{4}$ are the same, namely, the prime 19.

- $P_{4}$ terminates both $P_{11}$ and $P_{14}{ }^{2}$

- Three repdigits are imbedded in $P_{5}$.

- $P_{7}$ contains each of the ten decimal digits except 0 and 5.

- $P_{9}$ is the smallest perfect number to contain each of the nine nonzero digits at least once. It is zerofree.

- $P_{10}$ is the smallest perfect number to contain each of the ten decimal digits at least once.

\footnotetext{
${ }^{2}$ These contain respectively 65 and 366 digits.
} 


\subsection{Abundant and deficient numbers}

A number $n$ is abundant, perfect, or deficient if the sum of its proper divisors (including 1 but excluding $n$ itself) is greater than, equal to, or less than $n$. If we denote by $\sigma(n)$ the sum of all divisors of $n$, including 1 and $n$ itself, then $n$ is abundant, perfect, or deficient according as $\sigma(n)$ is greater than, equal to, or less than $2 n$. The advantage of using $\sigma(n)$ is that it can be easily computed if we know how $n$ factors into primes:

Abundant numbers up to 200:

$\begin{array}{ccccccccccccccc}12 & 18 & 20 & 24 & 30 & 36 & 40 & 42 & 48 & 54 & 56 & 60 & 66 & 70 & 72 \\ 78 & 80 & 84 & 88 & 90 & 96 & 100 & 102 & 104 & 108 & 112 & 114 & 120 & 126 & 132 \\ 138 & 140 & 144 & 150 & 156 & 160 & 162 & 168 & 174 & 176 & 180 & 186 & 192 & 196 & 198 \\ 200 & & & & & & & & & & & & & & \end{array}$

Deficient even numbers up to 200:

$\begin{array}{ccccccccccccccc}2 & 4 & 8 & 10 & 14 & 16 & 22 & 26 & 32 & 34 & 38 & 44 & 46 & 50 & 52 \\ 58 & 62 & 64 & 68 & 74 & 76 & 82 & 86 & 92 & 94 & 98 & 106 & 110 & 116 & 118 \\ 122 & 124 & 128 & 130 & 134 & 136 & 142 & 146 & 148 & 152 & 154 & 158 & 164 & 166 & 170 \\ 172 & 178 & 182 & 184 & 188 & 190 & 194 & & & & & & & & \end{array}$

All multiples of 6 are abundant. But not conversely. 20 is abundant. 945 is the first odd abundant number.

5775 and 5776 are the first pair of abundant numbers.

Pairs of consecutive abundant numbers up to 10,000 :

$\begin{array}{llll}5775,5776 & 5984,5985 & 7424,7425 & 11024,11025 \\ 21735,21736 & 21944,21945 & 26144,26145 & 27404,27405 \\ 39375,39376 & 43064,43065 & 49664,49665 & 56924,56925 \\ 58695,58696 & 61424,61425 & 69615,69616 & 70784,70785 \\ 76544,76545 & 77175,77176 & 79695,79696 & 81080,81081 \\ 81675,81676 & 82004,82005 & 84524,84525 & 84644,84645 \\ 89775,89776 & 91664,91665 & 98175,98176 & \ldots\end{array}$

The first triple of abundant numbers ${ }^{3}$

\begin{tabular}{llll}
$n$ & factorization & $\sigma(n)$ & $\sigma(n)-2 n$ \\
\hline 171078830 & $2 \cdot 5 \cdot 13 \cdot 23 \cdot 29 \cdot 1973$ & 358162560 & 16004900 \\
171078831 & $3^{3} \cdot 7 \cdot 11 \cdot 19 \cdot 61 \cdot 71$ & 342835200 & 677538 \\
171078832 & $2^{4} \cdot 31 \cdot 344917$ & 342158656 & 992
\end{tabular}

${ }^{3}$ Discovered in 1975 by Laurent Hodges and Reid, [Pickover, p. 364]. 
Appendix: Mersenne primes

Primes of the form $M_{k}=2^{k}-1$ are called Mersenne prime. The only known Mersenne primes are listed below.

\begin{tabular}{|ccl|c|cll|}
\hline$k$ & Year & Discoverer & $k$ & Year & Discoverer \\
\hline \hline 17 & 1588 & P.A.Cataldi & & 19 & 1588 & P.A.Cataldi \\
31 & 1750 & L.Euler & & 61 & 1883 & I.M.Pervushin \\
89 & 1911 & R.E.Powers & & 107 & 1913 & E.Fauquembergue \\
127 & 1876 & E.Lucas & & 521 & 1952 & R.M.Robinson \\
607 & 1952 & R.M.Robinson & & 1279 & 1952 & R.M.Robinson \\
2203 & 1952 & R.M.Robinson & & 2281 & 1952 & R.M.Robinson \\
3217 & 1957 & H.Riesel & & 4253 & 1961 & A.Hurwitz \\
4423 & 1961 & A.Hurwitz & & 11213 & 1963 & D.B.Gillies \\
9941 & 1963 & D.B.Gillies & & 21701 & 1978 & C.Noll, L.Nickel \\
19937 & 1971 & B.Tuckerman & & 44497 & 1979 & H.Nelson, D.Slowinski \\
23209 & 1979 & C.Noll & & 110503 & 1988 & W.N.Colquitt, L.Welsch \\
86243 & 1982 & D.Slowinski & & 216091 & 1985 & D.Slowinski \\
132049 & 1983 & D.Slowinski & 859433 & 1993 & D.Slowinski \\
756839 & 1992 & D.Slowinski,P.Gage & & 1398269 & 1996 & Armengaud, Woltman et al. \\
1257787 & 1996 & Slowinski and Gage & & 3021377 & 1998 & Clarkson, Woltman, \\
2976221 & 1997 & Spence, Woltman, et.al. & & & & Kurowski et. al. \\
6972593 & 1999 & Hajratwala, Woltman, & & 13466917 & 2001 & Cameron, Woltman, \\
& & Kurowski et. al. & & & Kurowski et. al. \\
\hline
\end{tabular}

$M_{13466917}$ has 4053946 digits and is the largest known prime. 


\section{Appendix: Three important number theoretic functions}

Let $n$ be a positive integer with prime factorization

$$
n=\prod_{i=1}^{k} p_{i}^{a_{i}}
$$

There are several important numbers associated with $n$.

1. The number of divisors of $n$ is

$$
d(n)=\prod_{i=1}^{k}\left(1+a_{i}\right)
$$

2. The sum of divisors of $n$, including 1 and $n$ itself, is

$$
\sigma(n)=\prod_{i=1}^{k} \frac{p_{i}^{a_{i}+1}}{p_{i}-1}
$$

3. The number of positive integers $<n$ which are relatively prime to $n$ is given by

$$
\phi(n)=n \prod_{i=1}^{k}\left(1-\frac{1}{p_{i}}\right) .
$$

These functions are all multiplicative in the sense that

$$
f(m n)=f(m) f(n) \quad \text { whenever } \operatorname{gcd}(m, n)=1 .
$$




\subsubsection{Appendix: Two enumerations of the rational numbers in $(\mathbf{0 , 1})$}

Consider two enumerations of the rational numbers in $(0,1)$.

$E_{1}$ lists them by increasing denominator, and for equal denominators, by increasing numerator. Thus,

$$
E_{1}: \frac{1}{2}, \frac{1}{3}, \frac{2}{3}, \frac{1}{4}, \frac{3}{4}, \frac{1}{5}, \frac{2}{5}, \frac{3}{5}, \frac{4}{5}, \frac{1}{6}, \frac{5}{6}, \ldots
$$

$E_{2}$, on the other hand, lists them by increasing sum of numerator and denominator, and for equal sums of terms, by increasing numerator. Thus,

$$
E_{2}: \frac{1}{2}, \frac{1}{3}, \frac{1}{4}, \frac{2}{3}, \frac{1}{5}, \frac{1}{6}, \frac{2}{5}, \frac{3}{4}, \frac{1}{7}, \frac{3}{5}, \frac{1}{8}, \ldots
$$

The fractions $\frac{1}{2}, \frac{1}{3}$, and $\frac{2}{5}$ occupy the same positions in both sequences. More generally, the rational number $\frac{m}{n}$ (with $m<n$ and $\operatorname{gcd}(m, n)=1$ ) occupies position

$$
\sum_{k=2}^{n-1} \phi(k)+\sum_{k=1}^{m} \chi(k, n)
$$

in enumeration $E_{1}$ and position

$$
\frac{1}{2} \sum_{k=3}^{m+n-1} \phi(k)+\sum_{k=1}^{m} \chi(k, m+n-k)
$$

in enumeration $E_{2}$. Here,

$$
\chi(m, n)= \begin{cases}1 & \text { if } \operatorname{gcd}(m, n)=1 \\ 0 & \text { otherwise }\end{cases}
$$

This was Computer Challenge 511 of Journal of Recreational Mathematics. The solution lists 10 of these. ${ }^{4}$

\begin{tabular}{ccccccccccc} 
Fraction & $\frac{1}{2}$ & $\frac{1}{3}$ & $\frac{2}{5}$ & $\frac{9}{23}$ & $\frac{30}{73}$ & $\frac{59}{143}$ & $\frac{67}{163}$ & $\frac{97}{235}$ & $\frac{197}{477}$ & $\frac{513}{1238}$ \\
\hline Position & 1 & 2 & 7 & 158 & 1617 & 6211 & 8058 & 16765 & 69093 & 465988
\end{tabular}

What is the next match? For $m+n \leq 20000$, I found four more:

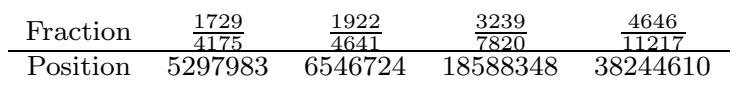

\footnotetext{
${ }^{4}$ Journal of Recreational Math., 10 (1977-78) 122-123.
} 


\section{Exercise}

1. The isle of Pythagora, while very sparsely populated, is capable of supporting a population of thirty million. On the 6th day of the 28th anniversary of his accession to the throne, the king of the island called a meeting of his 496 advisors to decide how to celebrate the auspicious occasion. They decided to divide the regal jewels among the people of the land. All the people, including the king the advisors, were lined up in a single file, and the jewels were distributed as follows.

Starting with the second in line, each person was given one jewel. Starting with the 4th in line, each second person was given two jewels.

Starting with the 6th in line, each third person was given three jewels.

Starting with the 8th in line, each fourth person was given four jewels, and so on.

The man at the extreme end of the line noticed that the number of jewels he received corresponded to his position in line.

How many people were there in Pythagora?

2. A minimum security prison contains 100 cells with one prisoner in each. The athletic young warden was ordered to free a certain number of these prisoners at his discretion, and this is how he did it.

First he walked along the row of cells opening every door. Starting at the beginning again, he shuts every second door. During his third walk, starting at the beginning, he stopped at every third door: if it was open he shut it, if it was shut he opened it. On his fourth walk he did the same, opening closed doors and closing open doors, except he did it for every fourth door. On his fifth walk he stopped at every fifth door, closing it if it was open and opening it if it was shut. And so on, until at last he had completed the full hundred walks.

The prisoners in cells whose doors were still open were freed.

Which were the lucky cells?

3. For a positive integer $n$, show that if $\sigma(n)$ is prime, then so is $d(n)$. 


\section{Chapter 25}

\section{Routh and Ceva theorems}

1 Routh theorem: an example

2 Routh theorem

3 Ceva theorem and coordinates of triangle centers centroid incenter Gergonne point Nagel point

Exercise 


\subsection{Routh theorem: an example}

Given a triangle $A B C, X, Y, Z$ are points on the side lines specified by the ratios of divisions

$$
B X: X C=2: 1, \quad C Y: Y A=5: 3, \quad A Z: Z B=3: 2 .
$$

The lines $A X, B Y, C Z$ bound a triangle $P Q R$. Suppose triangle $A B C$ has area $\triangle$. Find the area of triangle $P Q R$.

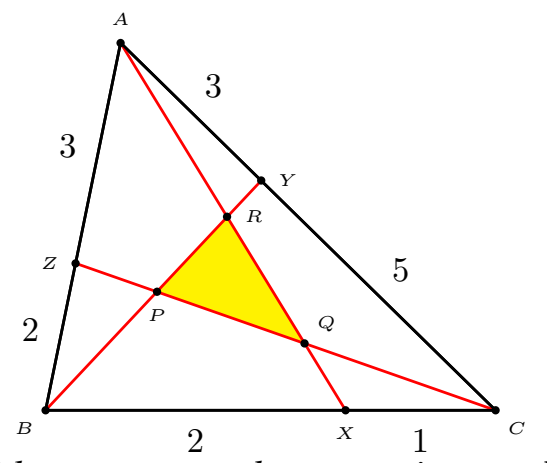

We make use of homogeneous barycentric coordinates with respect to $A B C$.

$$
X=(0: 1: 2), \quad Y=(5: 0: 3), \quad Z=(2: 3: 0) .
$$

Those of $P, Q, R$ can be worked out easily:

\begin{tabular}{|c|c|c|}
\hline$P=B Y \cap C Z$ & $Q=C Z \cap A X$ & $R=A X \cap B Y$ \\
\hline \hline$Y=(5: 0: 3)$ & $Z=(2: 3: 0)$ & $X=(0: 1: 2)$ \\
$Z=(2: 3: 0)$ & $X=(0: 1: 2)$ & $Y=(5: 0: 3)$ \\
\hline$P=(10: 15: 6)$ & $Q=(2: 3: 6)$ & $R=(10: 3: 6)$ \\
\hline
\end{tabular}

This means that the absolute barycentric coordinates of $X, Y, Z$ are

$$
P=\frac{1}{31}(10 A+15 B+6 C), \quad Q=\frac{1}{11}(2 A+3 B+6 C), \quad R=\frac{1}{19}(10 A+3 B+6 C)
$$

The area of triangle $P Q R$

$$
=\frac{1}{31 \cdot 11 \cdot 19}\left|\begin{array}{ccc}
10 & 15 & 6 \\
2 & 3 & 6 \\
10 & 3 & 6
\end{array}\right| \cdot \Delta=\frac{576}{6479} \triangle .
$$




\subsection{Routh theorem}

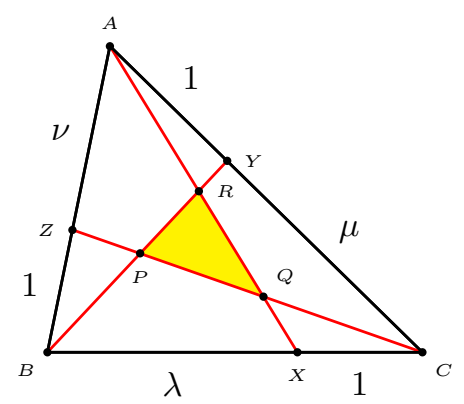

We make use of homogeneous barycentric coordinates with respect to $A B C$.

$$
X=(0: 1: \lambda), \quad Y=(\mu: 0: 1), \quad Z=(1: \nu: 0) .
$$

Those of $P, Q, R$ can be worked out easily:

\begin{tabular}{|c|c|c|}
\hline$P=B Y \cap C Z$ & $Q=C Z \cap A X$ & $R=A X \cap B Y$ \\
\hline \hline$Y=(\mu: 0: 1)$ & $Z=(1: \nu: 0)$ & $X=(0: 1: \lambda)$ \\
$Z=(1: \nu: 0)$ & $X=(0: 1: \lambda)$ & $Y=(\mu: 0: 1)$ \\
\hline$P=(\mu: \mu \nu: 1)$ & $Q=(1: \nu: \nu \lambda)$ & $R=(\lambda \mu: 1: \lambda)$ \\
\hline
\end{tabular}

This means that the absolute barycentric coordinates of $X, Y, Z$ are

$$
\begin{aligned}
P & =\frac{1}{\mu \nu+\mu+1}(\mu A+\mu \nu B+C), \\
Q & =\frac{1}{\nu \lambda+\nu+1}(A+\nu B+\nu \lambda C), \\
R & =\frac{1}{\lambda \mu+\lambda+1}(\lambda \mu A+B+\lambda C) .
\end{aligned}
$$

From these,

$$
\begin{aligned}
\operatorname{Area}(P Q R) & =\frac{\left|\begin{array}{ccc}
\mu & \mu \nu & 1 \\
1 & \nu & \nu \lambda \\
\lambda \mu & 1 & \lambda
\end{array}\right|}{(\mu \nu+\mu+1)(\nu \lambda+\nu+1)(\lambda \mu+\lambda+1)} \cdot \triangle \\
& =\frac{(\lambda \mu \nu-1)^{2}}{(\mu \nu+\mu+1)(\nu \lambda+\nu+1)(\lambda \mu+\lambda+1)} \cdot \triangle .
\end{aligned}
$$




\subsection{Ceva Theorem}

Theorem 25.1 (Ceva). Let $X, Y, Z$ be points on the lines $B C, C A, A B$ respectively. The lines $A X, B Y, C Z$ are concurrent if and only if

$$
\frac{B X}{X C} \cdot \frac{C Y}{Y A} \cdot \frac{A Z}{Z B}=1
$$

If this condition is satisfied, the homogeneous barycentric coordinates of the common point of $A X, B Y, C Z$ can be written down by combining the coordinates of $X, Y, Z$.

\section{Example: centroid}

If $A X, B Y, C Z$ are the medians, the intersection is the centroid $G$ :

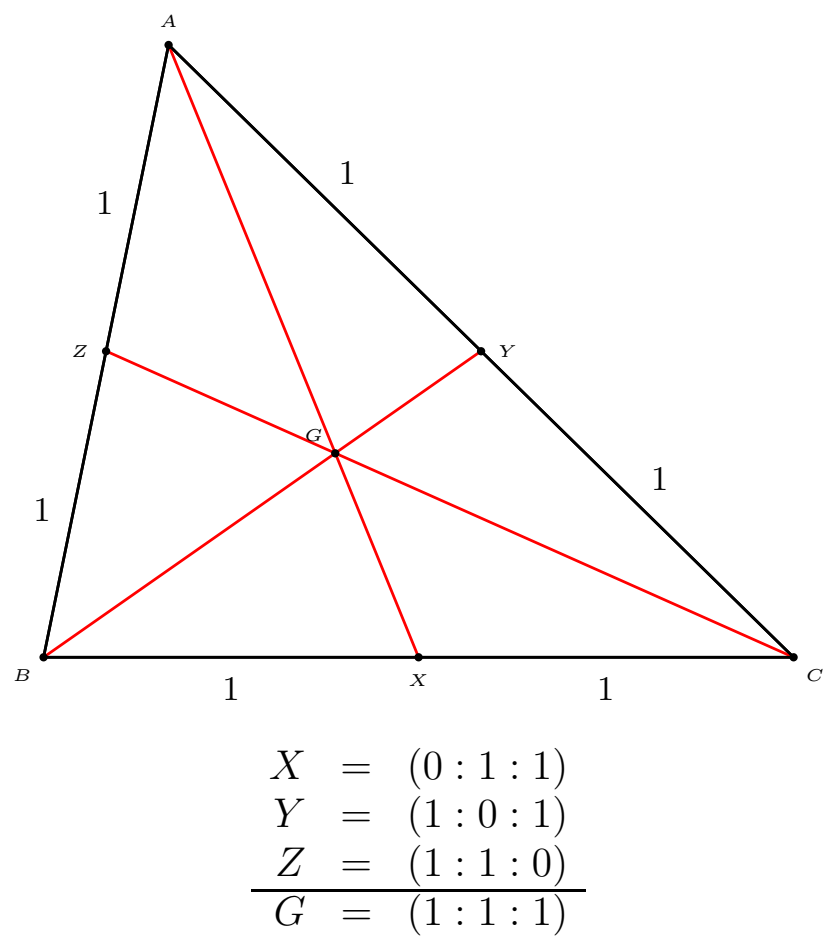




\section{Example: incenter}

If $A X, B Y, C Z$ are the angle bisectors, the intersection is the incenter $I$ :

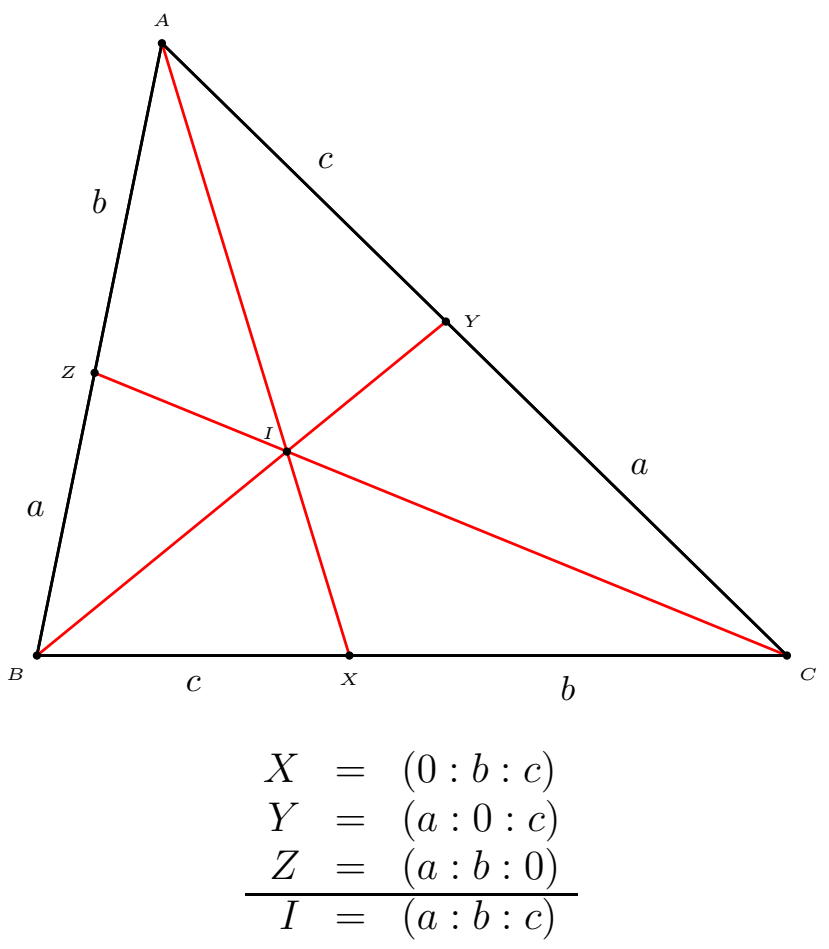




\section{Example: Gergonne point}

If $X, Y, Z$ are the points of tangency of the incircle with the sidelines, the lines $A X, B Y, C Z$ intersect at the Gergonne point.

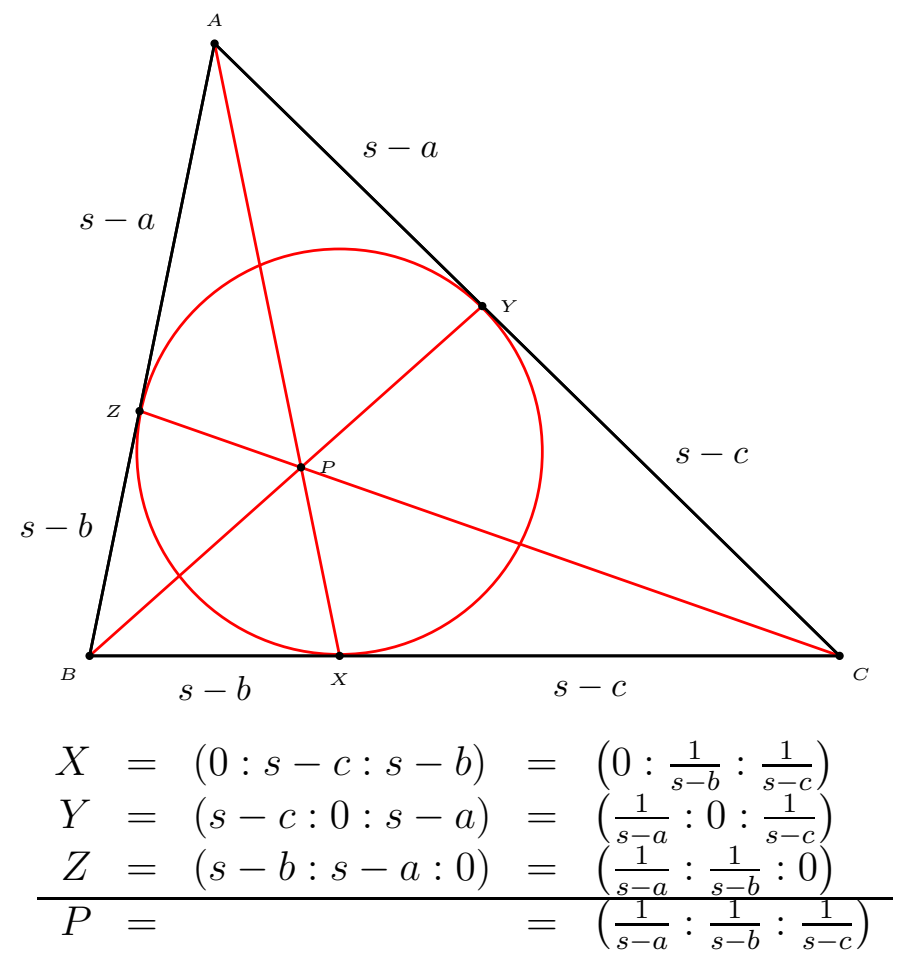




\section{Example: Nagel point}

If $X, Y, Z$ are the points of tangency of the excircles with the respective sidelines, the lines $A X, B Y, C Z$ intersect at the Nagel point.

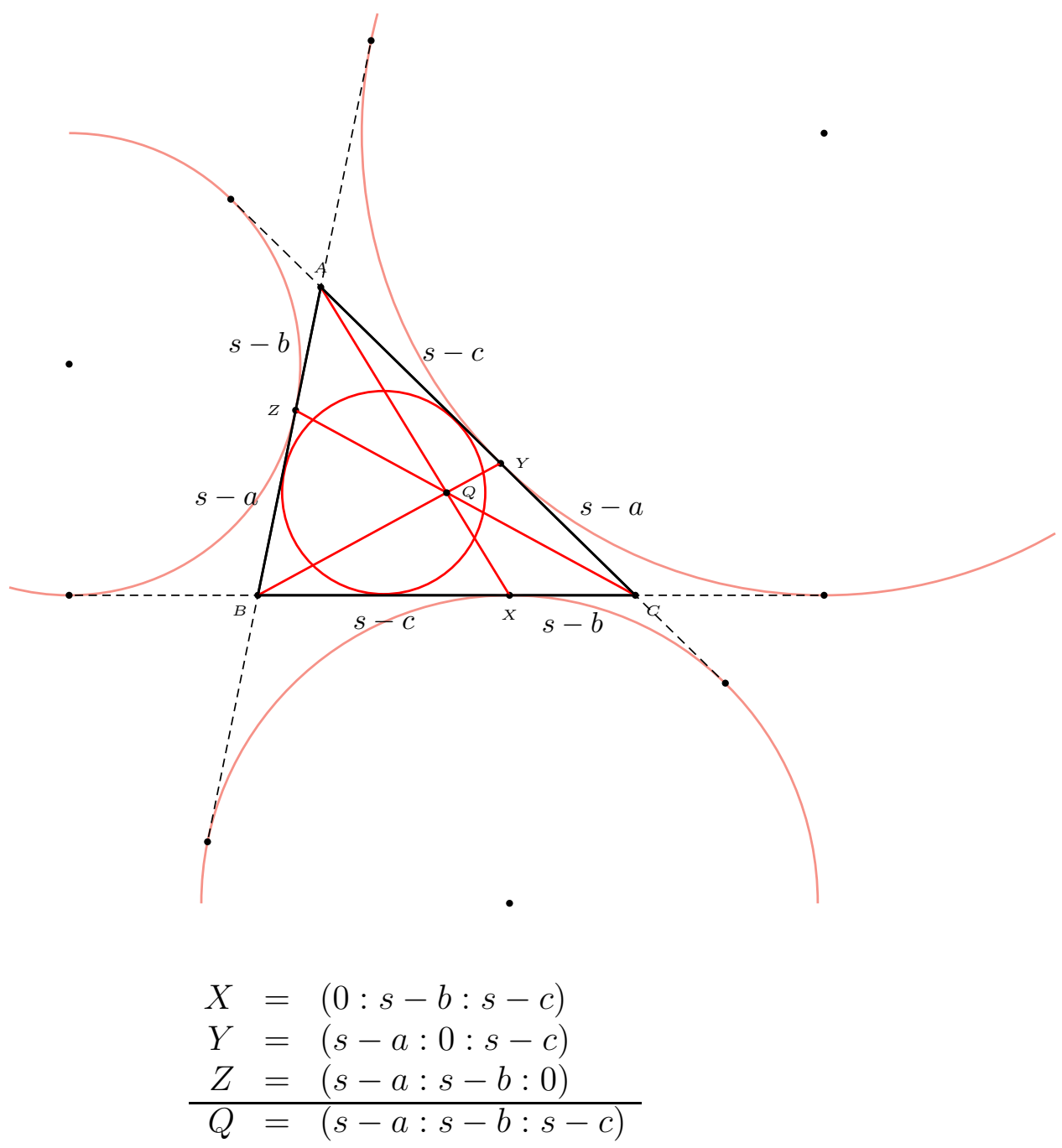




\section{Exercise}

1. Calculate the area of triangle $P Q R$ given
(a) $\lambda=\mu=\nu=2$.
(b) $\lambda=1, \mu=7, \nu=4$;
(c) $\lambda=3, \mu=7, \nu=6$.

2. Calculate the homogeneous barycentric coordinates of the orthocenter of triangle $A B C$.

\section{Project}

In the Routh formula, how should one choose integer values for $\lambda, \mu$, and $\nu$ so that the area of triangle $P Q R$ is $\frac{1}{n}$ of that of triangle $A B C$ for an integer $n$ ? 


\section{Appendix}

We give those values of $\lambda, \mu, \nu$ with numerators and denominators $<10$ for which the area of triangle $P Q R$ is $k$ times that of $A B C$ with the numerator and denominator of $k$ less than 10 .

\begin{tabular}{|c|c|c|c|c|c|c|c|c|c|c|c|c|c|c|c|}
\hline & $\mu$ & $\nu$ & $k$ & $\bar{\lambda}$ & $\mu$ & $\nu$ & $k$ & $\lambda$ & $\mu$ & $\nu$ & $k$ & $\lambda$ & $\mu$ & $\nu$ & $k$ \\
\hline & $\frac{4}{5}$ & $\frac{9}{8}$ & $\frac{1}{9}$ & $\frac{1}{8}$ & $\frac{1}{3}$ & $\frac{8}{5}$ & $\frac{T}{7}$ & $\frac{1}{8}$ & $\frac{3}{8}$ & $\frac{1}{2}$ & $\frac{1}{3}$ & $\frac{1}{8}$ & $\frac{9}{5}$ & $\frac{4}{9}$ & $\frac{T}{9}$ \\
\hline & $\frac{1}{3}$ & $\frac{1}{6}$ & $\frac{1}{2}$ & $\frac{1}{7}$ & $\frac{2}{5}$ & $\frac{7}{6}$ & $\frac{1}{6}$ & $\frac{1}{7}$ & $\frac{0}{1}$ & $\frac{1}{4}$ & $\frac{1}{4}$ & $\frac{1}{6}$ & $\frac{1}{7}$ & $\frac{1}{3}$ & $\frac{1}{2}$ \\
\hline$\overline{6}$ & $\frac{1}{5}$ & $\frac{6}{5}$ & $\frac{2}{9}$ & $\frac{1}{6}$ & $\frac{1}{2}$ & $\frac{3}{4}$ & $\frac{1}{5}$ & $\frac{1}{6}$ & $\frac{7}{8}$ & $\frac{6}{7}$ & $\frac{1}{9}$ & $\frac{1}{6}$ & 1 & $\frac{2}{3}$ & $\frac{1}{8}$ \\
\hline$\frac{1}{6}$ & $\frac{7}{5}$ & $\frac{2}{7}$ & $\frac{1}{6}$ & $\frac{1}{5}$ & $\frac{6}{7}$ & $\frac{1}{2}$ & $\frac{1}{6}$ & $\frac{1}{5}$ & $\frac{6}{5}$ & $\frac{1}{6}$ & $\frac{2}{9}$ & $\frac{1}{4}$ & $\frac{1}{7}$ & 1 & $\frac{1}{4}$ \\
\hline$\frac{1}{4}$ & $\frac{1}{4}$ & $\frac{1}{4}$ & $\frac{3}{7}$ & $\frac{1}{4}$ & $\frac{1}{3}$ & $\frac{4}{3}$ & $\frac{1}{8}$ & $\frac{1}{4}$ & $\frac{5}{8}$ & $\frac{2}{5}$ & $\frac{2}{9}$ & $\frac{1}{4}$ & 1 & $\frac{4}{7}$ & $\frac{1}{9}$ \\
\hline$\frac{2}{7}$ & $\frac{1}{6}$ & $\frac{7}{5}$ & $\frac{1}{6}$ & $\frac{1}{3}$ & $\frac{1}{6}$ & $\frac{1}{7}$ & $\frac{1}{2}$ & $\frac{1}{3}$ & $\frac{1}{2}$ & $\frac{3}{5}$ & $\frac{1}{6}$ & $\frac{1}{3}$ & $\frac{8}{7}$ & $\frac{3}{8}$ & $\frac{1}{9}$ \\
\hline$\frac{1}{3}$ & $\frac{4}{3}$ & $\frac{1}{4}$ & $\frac{1}{8}$ & $\frac{1}{3}$ & $\frac{8}{5}$ & $\frac{1}{8}$ & $\frac{1}{7}$ & $\frac{3}{8}$ & $\frac{1}{3}$ & $\frac{8}{7}$ & $\frac{1}{9}$ & $\frac{3}{8}$ & $\frac{1}{2}$ & $\frac{1}{8}$ & $\frac{1}{3}$ \\
\hline$\frac{5}{8}$ & $\frac{5}{6}$ & $\frac{1}{2}$ & $\frac{1}{9}$ & $\frac{2}{5}$ & $\frac{1}{4}$ & $\frac{5}{8}$ & $\frac{2}{9}$ & $\frac{2}{5}$ & $\frac{7}{6}$ & $\frac{1}{7}$ & $\frac{1}{6}$ & $\frac{3}{7}$ & $\frac{2}{3}$ & $\frac{1}{2}$ & $\frac{1}{8}$ \\
\hline$\frac{4}{9}$ & $\frac{6}{8}$ & $\frac{9}{5}$ & $\frac{9}{9}$ & $\frac{5}{2}$ & $\frac{4}{8}$ & $\frac{3}{8}$ & $\frac{1}{3}$ & $\frac{5}{2}$ & $\frac{6}{5}$ & $\frac{6}{7}$ & $\frac{6}{6}$ & $\frac{1}{2}$ & $\frac{3}{8}$ & $\frac{5}{6}$ & $\frac{8}{\frac{1}{9}}$ \\
\hline$\frac{1}{2}$ & $\frac{3}{7}$ & $\frac{2}{3}$ & $\frac{1}{8}$ & $\frac{1}{2}$ & $\frac{1}{2}$ & $\frac{1}{2}$ & $\frac{1}{7}$ & $\frac{1}{2}$ & $\frac{3}{5}$ & $\frac{1}{3}$ & $\frac{1}{6}$ & $\frac{1}{2}$ & $\frac{3}{4}$ & $\frac{1}{6}$ & $\frac{1}{5}$ \\
\hline$\frac{0}{9}$ & 8 & $\frac{y}{4}$ & $\frac{1}{9}$ & $\frac{4}{7}$ & $\frac{1}{4}$ & 1 & $\frac{1}{a}$ & $\frac{3}{5}$ & $\frac{1}{2}$ & $\frac{1}{2}$ & $\frac{1}{6}$ & $\frac{5}{8}$ & $\frac{2}{5}$ & $\frac{1}{4}$ & $\frac{2}{9}$ \\
\hline$\frac{5}{8}$ & 3 & 8 & $\frac{1}{7}$ & $\frac{2}{3}$ & $\frac{1}{6}$ & 1 & $\frac{1}{8}$ & $\frac{2}{3}$ & $\frac{1}{2}$ & $\frac{3}{7}$ & $\frac{1}{8}$ & $\frac{5}{7}$ & 6 & $\frac{7}{2}$ & $\frac{1}{6}$ \\
\hline$\frac{3}{4}$ & $\frac{1}{6}$ & $\frac{1}{2}$ & $\frac{1}{5}$ & $\frac{3}{4}$ & 3 & 4 & $\frac{1}{8}$ & $\frac{4}{5}$ & $\frac{9}{8}$ & $\frac{1}{9}$ & $\frac{1}{9}$ & $\frac{5}{6}$ & $\frac{1}{2}$ & $\frac{3}{8}$ & $\frac{1}{9}$ \\
\hline$\frac{5}{6}$ & 5 & 6 & $\frac{2}{9}$ & $\frac{6}{7}$ & $\frac{1}{6}$ & $\frac{7}{8}$ & $\frac{1}{9}$ & $\frac{6}{7}$ & $\frac{1}{2}$ & $\frac{1}{5}$ & $\frac{1}{6}$ & $\frac{6}{7}$ & $\frac{5}{2}$ & 7 & $\frac{1}{6}$ \\
\hline$\frac{7}{8}$ & $\frac{6}{7}$ & $\frac{1}{6}$ & $\frac{1}{9}$ & $\frac{7}{8}$ & 3 & $\frac{8}{3}$ & $\frac{1}{9}$ & $\frac{8}{9}$ & $\frac{5}{4}$ & 9 & $\frac{1}{9}$ & 1 & $\frac{1}{4}$ & $\frac{1}{7}$ & $\frac{1}{4}$ \\
\hline$\frac{1}{1}$ & $\frac{4}{7}$ & $\frac{1}{4}$ & $\frac{1}{9}$ & 1 & $\frac{2}{3}$ & $\frac{1}{6}$ & $\frac{1}{8}$ & 1 & 4 & $\frac{7}{4}$ & $\frac{1}{9}$ & 1 & 6 & $\frac{3}{2}$ & $\frac{1}{8}$ \\
\hline 1 & 7 & 4 & $\frac{1}{4}$ & $\frac{9}{8}$ & $\frac{1}{9}$ & $\frac{4}{5}$ & $\frac{1}{9}$ & $\frac{8}{7}$ & $\frac{3}{8}$ & $\frac{1}{3}$ & $\frac{1}{9}$ & $\frac{8}{7}$ & 6 & $\frac{7}{6}$ & $\frac{1}{9}$ \\
\hline$\frac{7}{6}$ & $\frac{1}{7}$ & $\frac{2}{5}$ & $\frac{1}{6}$ & $\frac{7}{6}$ & $\frac{8}{7}$ & 6 & $\frac{1}{9}$ & $\frac{7}{6}$ & 5 & 2 & $\frac{1}{6}$ & $\frac{6}{5}$ & $\frac{1}{6}$ & $\frac{1}{5}$ & $\frac{2}{9}$ \\
\hline$\frac{6}{5}$ & $\frac{8}{3}$ & 2 & $\frac{1}{9}$ & $\frac{5}{4}$ & 9 & $\frac{8}{9}$ & $\frac{1}{9}$ & $\frac{4}{3}$ & $\frac{1}{4}$ & $\frac{1}{3}$ & $\frac{1}{8}$ & $\frac{4}{3}$ & 2 & 6 & $\frac{1}{5}$ \\
\hline$\frac{7}{5}$ & $\frac{2}{7}$ & $\frac{1}{6}$ & $\frac{1}{6}$ & $\frac{3}{2}$ & 1 & 6 & $\frac{1}{8}$ & $\frac{3}{2}$ & $\frac{7}{3}$ & 2 & $\frac{1}{8}$ & $\frac{8}{5}$ & $\frac{1}{8}$ & $\frac{1}{3}$ & $\frac{1}{7}$ \\
\hline$\frac{8}{5}$ & 4 & $\frac{5}{2}$ & $\frac{2}{9}$ & $\frac{5}{3}$ & 2 & 3 & $\frac{1}{6}$ & $\frac{7}{4}$ & 1 & 4 & $\frac{1}{9}$ & $\frac{9}{5}$ & $\frac{4}{9}$ & $\frac{1}{8}$ & $\frac{T}{9}$ \\
\hline$\frac{2}{2}$ & $\frac{7}{6}$ & 5 & $\frac{1}{6}$ & 2 & $\frac{6}{5}$ & $\frac{8}{3}$ & $\frac{1}{9}$ & 2 & $\frac{3}{2}$ & $\frac{7}{3}$ & $\frac{11}{8}$ & 2 & 2 & 2 & $\frac{1}{7}$ \\
\hline 2 & $\frac{8}{3}$ & 8 & $\frac{1}{3}$ & 2 & 3 & $\frac{5}{3}$ & $\frac{1}{6}$ & 2 & 6 & $\frac{4}{3}$ & $\frac{1}{5}$ & $\frac{9}{4}$ & $\frac{5}{9}$ & 8 & $\frac{1}{a}$ \\
\hline$\frac{7}{3}$ & 2 & $\frac{3}{2}$ & $\frac{1}{8}$ & $\frac{5}{2}$ & $\frac{8}{5}$ & 4 & $\frac{2}{9}$ & $\frac{5}{2}$ & 7 & $\frac{6}{7}$ & $\frac{1}{6}$ & $\frac{4}{3}$ & $\frac{7}{8}$ & 3 & $\frac{1}{9}$ \\
\hline$\frac{8}{\frac{8}{3}}$ & 2 & $\frac{6}{5}$ & $\frac{?}{a}$ & $\frac{8}{2}$ & 8 & 2 & $\frac{1}{3}$ & 3 & $\frac{5}{2}$ & 2 & $\frac{1}{\frac{1}{6}}$ & 3 & $\frac{8}{2}$ & $\frac{7}{8}$ & $\frac{1}{a}$ \\
\hline 3 & 4 & $\frac{3}{4}$ & $\frac{1}{8}$ & 3 & 7 & 6 & $\frac{1}{2}$ & 3 & 8 & $\frac{5}{8}$ & $\frac{1}{7}$ & $\frac{7}{2}$ & $\frac{5}{7}$ & 6 & $\frac{1}{6}$ \\
\hline 4 & $\frac{3}{4}$ & 3 & $\frac{9}{\frac{1}{2}}$ & 4 & 1 & 7 & $\frac{1}{4}$ & 4 & $7 \frac{7}{4}$ & 1 & $\frac{1}{a}$ & 4 & $\frac{5}{\frac{5}{2}}$ & $\frac{8}{5}$ & $\frac{2}{a}$ \\
\hline$\overline{4}$ & 4 & 4 & $\frac{3}{7}$ & 5 & 2 & $\frac{7}{6}$ & $\frac{1}{6}$ & 5 & 6 & $\frac{5}{6}$ & $\frac{2}{9}$ & 6 & $\frac{5}{6}$ & 5 & $\frac{2}{a}$ \\
\hline 6 & $\frac{7}{6}$ & $\frac{8}{7}$ & $\frac{1}{9}$ & 6 & $\frac{4}{3}$ & 2 & $\frac{1}{5}$ & 6 & $\frac{3}{2}$ & 1 & $\frac{1}{8}$ & 6 & 3 & 7 & $\frac{1}{2}$ \\
\hline 6 & $\frac{7}{2}$ & $\frac{5}{7}$ & $\frac{1}{6}$ & 7 & $\frac{6}{7}$ & $\frac{5}{2}$ & $\frac{1}{6}$ & 7 & 4 & 1 & $\frac{1}{4}$ & 7 & 6 & 3 & $\frac{1}{2}$ \\
\hline 8 & $\frac{5}{8}$ & 3 & $\frac{1}{7}$ & 8 & 2 & $\frac{8}{3}$ & $\frac{1}{3}$ & 8 & $\frac{9}{4}$ & $\frac{5}{9}$ & $\frac{1}{9}$ & 9 & $\frac{8}{9}$ & $\frac{5}{4}$ & $\frac{1}{9}$ \\
\hline
\end{tabular}




\section{Chapter 26}

\section{The excircles}

1 Feuerbach theorem

2 A relation among radii

3 The circumcircle of the excentral triangle

4 The radical circle of the excircles

5 Apollonius circle: the circular hull of the excircles

Appendix: Three mutually orthogonal circles with given centers 
712

The excircles

26.1 Feuerbach theorem

The nine-point circle is tangent internally to the incircle and externally to each of the excircles.

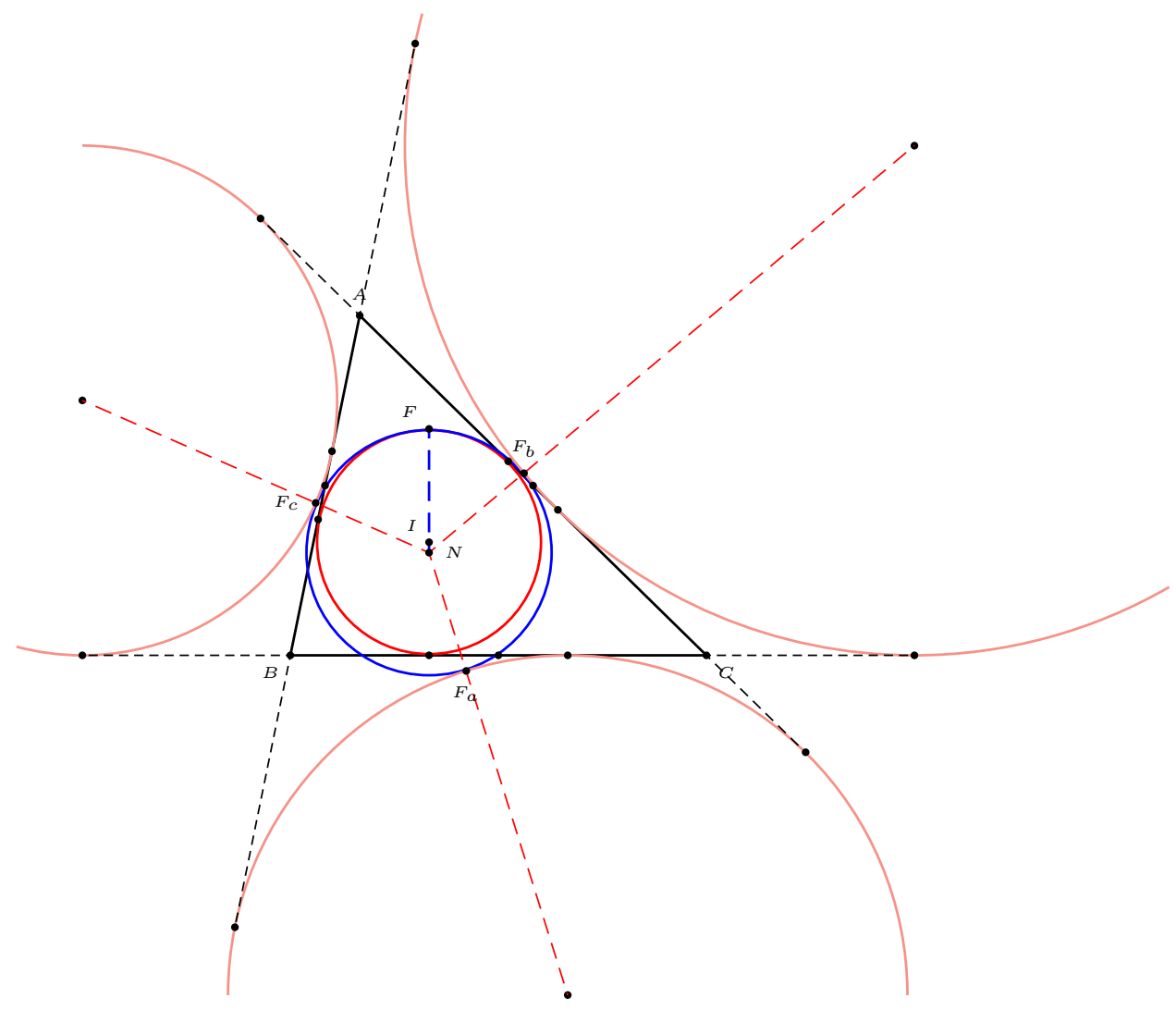




\subsection{A relation among the radii}

$$
r_{a}+r_{b}+r_{c}=4 R+r
$$

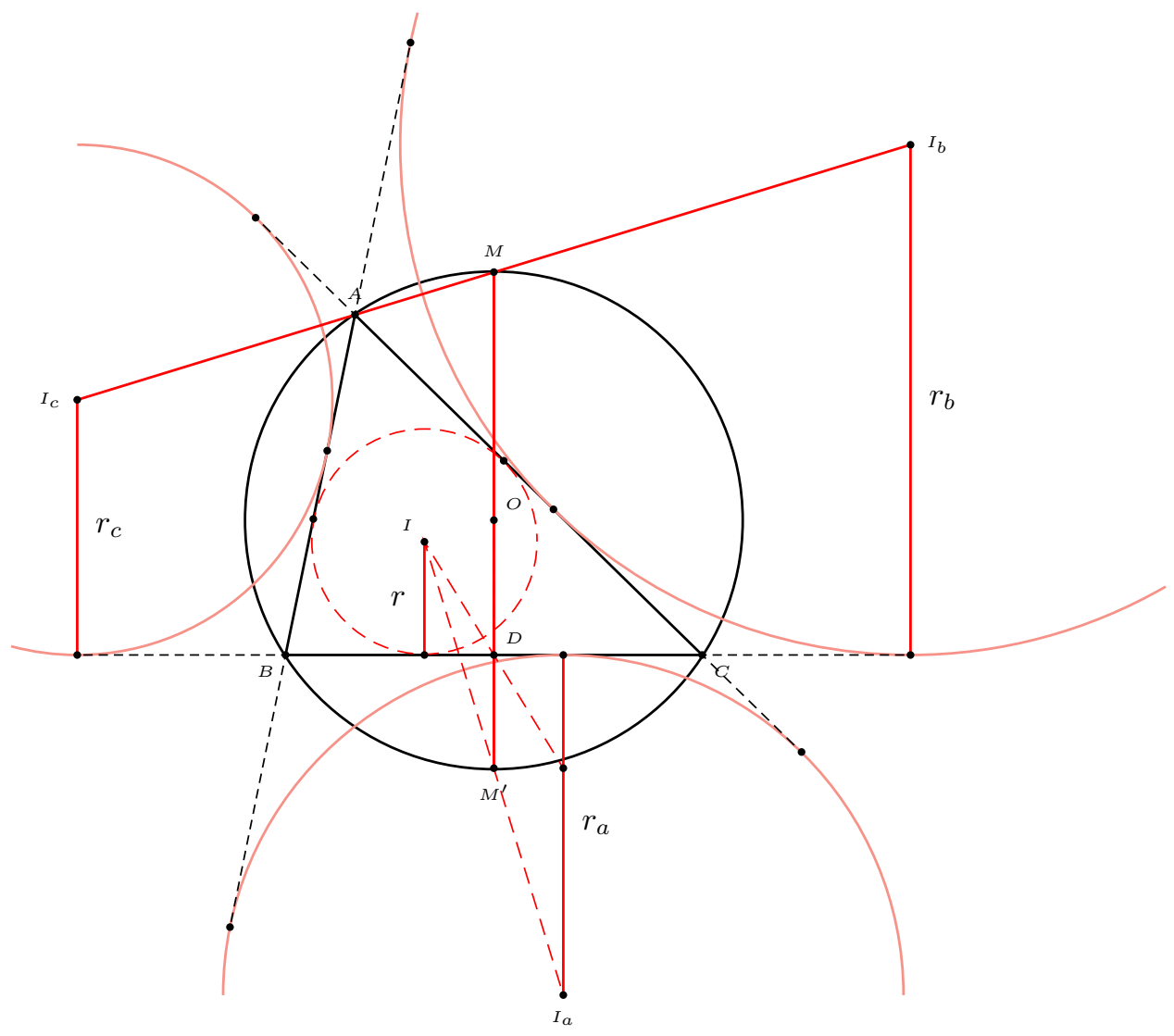

$$
\begin{aligned}
r_{a}-r & =2 D M^{\prime}, \\
r_{b}+r_{c} & =2 M D=2\left(2 R-D M^{\prime}\right) ; \\
r_{a}+r_{b}+r_{c}-r & =4 R .
\end{aligned}
$$




\subsection{The circumcircle of the excentral triangle}

The circle through the excenters has center at the reflection of the incenter in the circumcenter, and radius twice the circumradius.

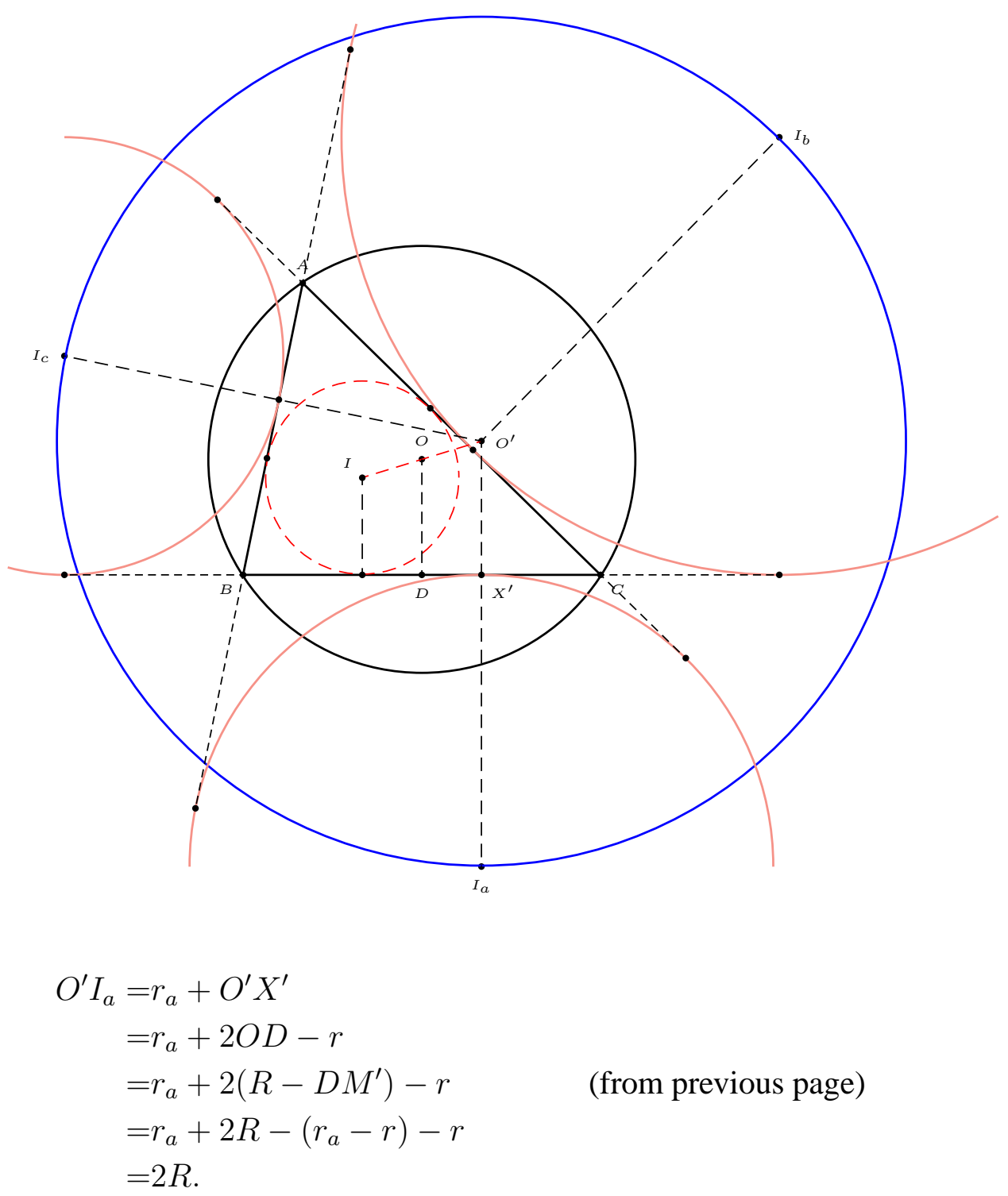

Similarly, $O^{\prime} I_{b}=O^{\prime} I_{c}=2 R$. 


\subsection{The radical circle of the excircles}

The circle orthogonal to each of the excircles has center at the Spieker point, the incenter of the medial triangle. Its radius is $\frac{1}{2} \sqrt{r^{2}+s^{2}}$.

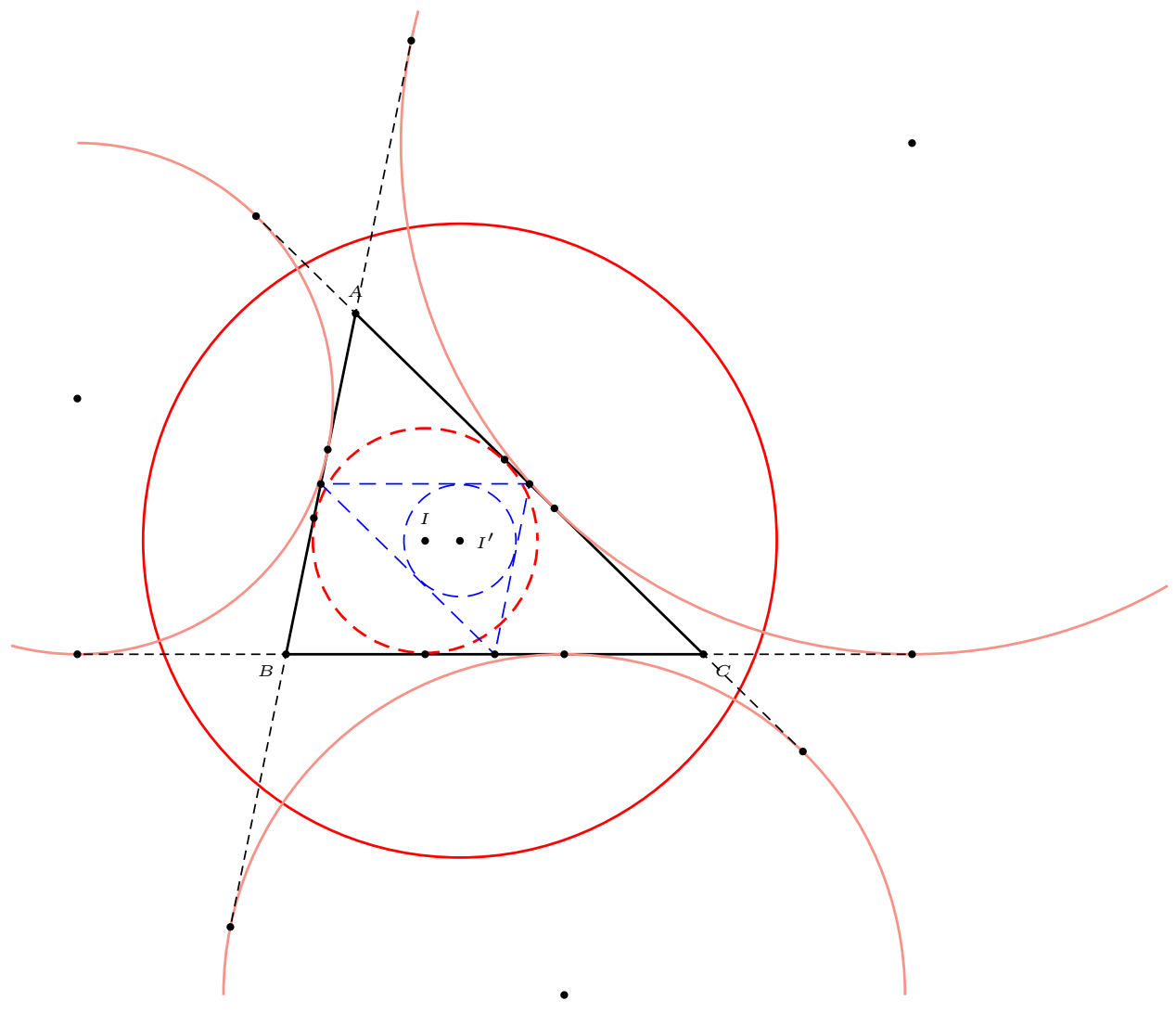




\subsection{Apollonius circle: the circular hull of the excircles}

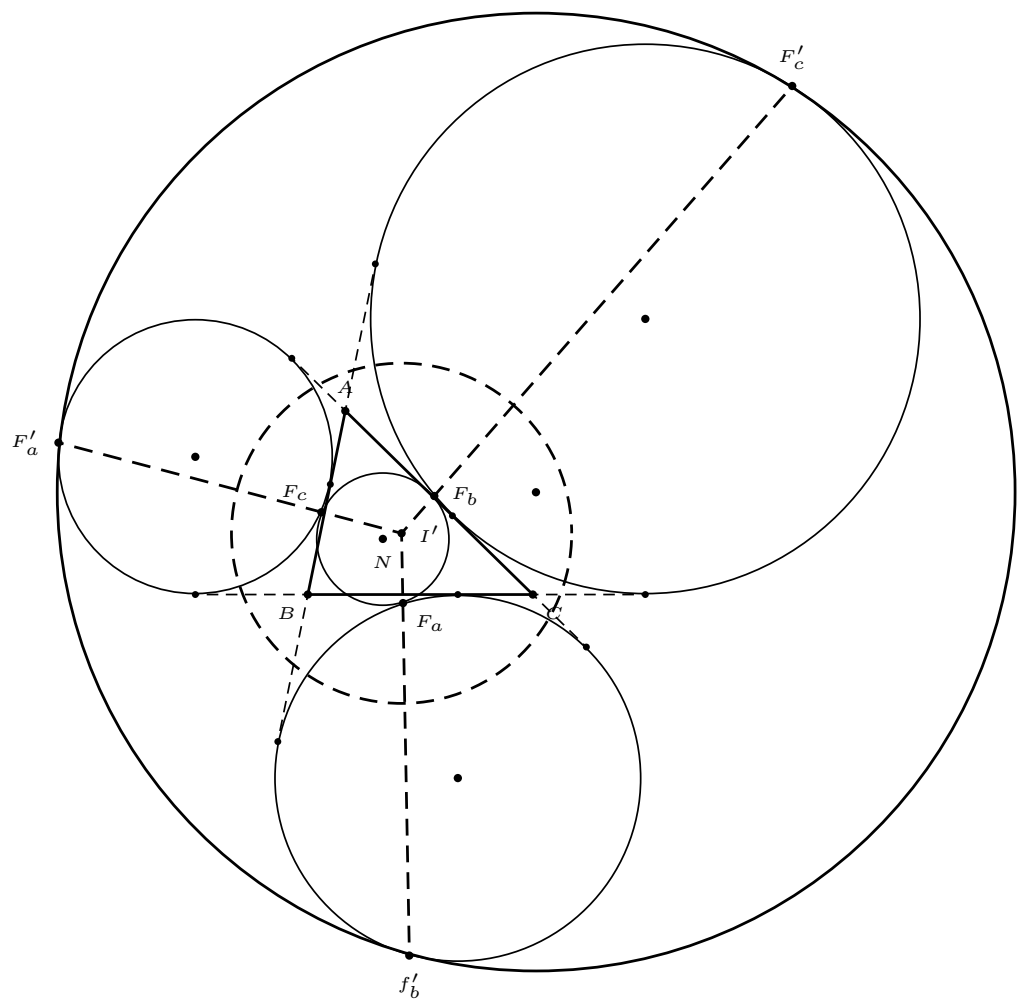




\section{Appendix: Three mutually orthogonal circles with given centers}

Given three points $A, B, C$ that form an acute-angled triangle, construct three circles with these points as centers that are mutually orthogonal to each other.

\section{Solution}

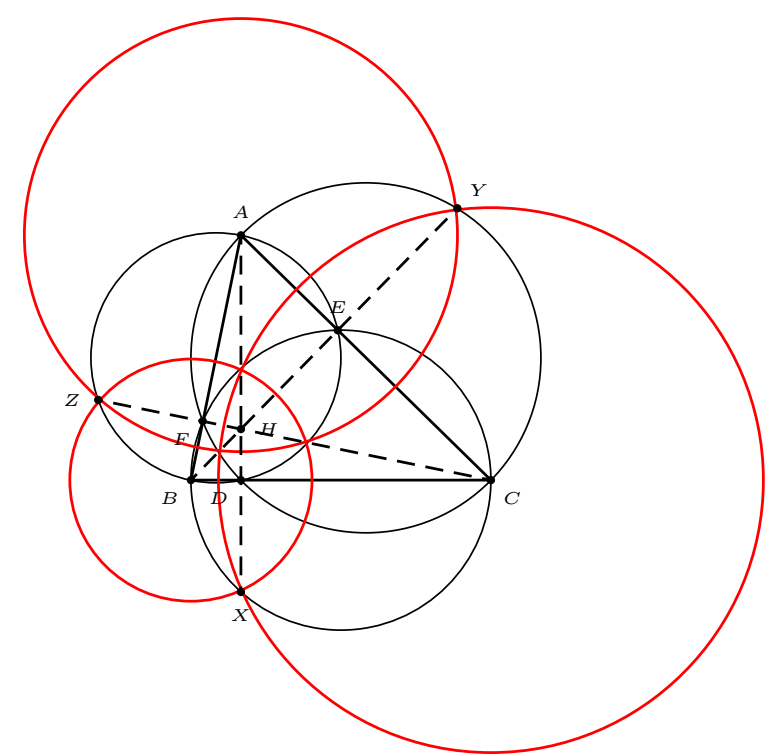

Let $B C=a, C A=b$, and $A B=c$. If these circles have radii $r_{a}, r_{b}, r_{c}$ respectively, then

$$
r_{b}^{2}+r_{c}^{2}=a^{2}, \quad r_{c}^{2}+r_{a}^{2}=b^{2}, \quad r_{a}^{2}+r_{b}^{2}=c^{2} .
$$

From these,

$r_{a}^{2}=\frac{1}{2}\left(b^{2}+c^{2}-a^{2}\right), \quad r_{b}^{2}=\frac{1}{2}\left(c^{2}+a^{2}-b^{2}\right), \quad r_{c}^{2}=\frac{1}{2}\left(a^{2}+b^{2}-c^{2}\right)$.

These are all positive since $A B C$ is an acute triangle. Consider the perpendicular foot $E$ of $B$ on $A C$. Note that $A E=c \cos A$, so that $r_{a}^{2}=\frac{1}{2}\left(b^{2}+c^{2}-a^{2}\right)=b c \cos A=A C \cdot A E$. It follows if we extend $B E$ to intersect at $Y$ the semicircle constructed externally on the side $A C$ as diameter, then, $A Y^{2}=A C \cdot A E=r_{a}^{2}$. Therefore we have the following simple construction of these circles. (1) With each side as diameter, construct a semicircle externally of the triangle. (2) Extend the altitudes of the triangle to intersect the semicircles on the same side. Label these $X, Y, Z$ on the semicircles on $B C, C A, A B$ respectively. These satify $A Y=A Z, B Z=B X$, and $C X=C Y$. (3) The circles $A(Y), B(Z)$ and $C(X)$ are mutually orthogonal to each other. 


\section{Chapter 27}

\section{Figurate numbers}

\subsection{Triangular numbers}

The $n$th triangular number is

$$
T_{n}=1+2+3+\cdots+n=\frac{1}{2} n(n+1) .
$$

The first few of these are $1,3,6,10,15,21,28,36,45,55, \ldots$

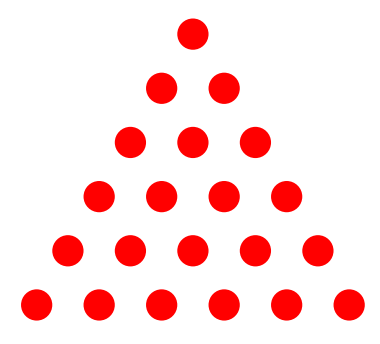

\subsection{Special triangular numbers}

\section{Triangular numbers which are squares}

The $n$-th triangular number $T_{n}=\frac{1}{2} n(n+1)$ is a square, say, $m^{2}$ if and only if

$$
\begin{gathered}
(2 n+1)^{2}-2(2 m)^{2}=1 . \\
\left(\begin{array}{c}
n_{k+1} \\
m_{k+1}
\end{array}\right)=\left(\begin{array}{ll}
3 & 4 \\
2 & 3
\end{array}\right)\left(\begin{array}{c}
n_{k} \\
m_{k}
\end{array}\right)+\left(\begin{array}{l}
1 \\
1
\end{array}\right), \quad\left(\begin{array}{c}
n_{1} \\
m_{1}
\end{array}\right)=\left(\begin{array}{l}
1 \\
1
\end{array}\right) .
\end{gathered}
$$


Find the first few triangular numbers which are squares:

\begin{tabular}{cccccccc}
$k$ & 1 & 2 & 3 & 4 & 5 & 6 & 7 \\
\hline$n_{k}$ & 1 & 8 & 49 & & & & \\
$m_{k}$ & 1 & 6 & 35 & & & & \\
$T_{n}$ & 1 & 36 & 1225 & & & &
\end{tabular}

Palindromic triangular numbers

\begin{tabular}{|cc||cc||cc|}
\hline$n$ & $T_{n}$ & $n$ & $T_{n}$ & $n$ & $T_{n}$ \\
\hline \hline 1 & 1 & 109 & 5995 & 3185 & 5073705 \\
2 & 3 & 132 & 8778 & 3369 & 5676765 \\
3 & 6 & 173 & 15051 & 3548 & 6295926 \\
10 & 55 & 363 & 66066 & 8382 & 35133153 \\
11 & 66 & 1111 & 617716 & 11088 & 61477416 \\
18 & 171 & 1287 & 828828 & 18906 & 178727871 \\
34 & 595 & 1593 & 1269621 & 57166 & 1634004361 \\
36 & 666 & 1833 & 1680861 & 102849 & 5289009825 \\
77 & 3003 & 2662 & 3544453 & 111111 & 6172882716 \\
\hline \multicolumn{5}{|c}{$T_{11111111}=61728399382716}$.
\end{tabular}




\subsection{Pentagonal numbers}

The pentagonal numbers are the sums of the arithmetic progression

$$
1+4+7+\cdots+(3 n-2)+\cdots
$$

The $n$th pentagonal number is $P_{n}=\frac{1}{2} n(3 n-1)$.

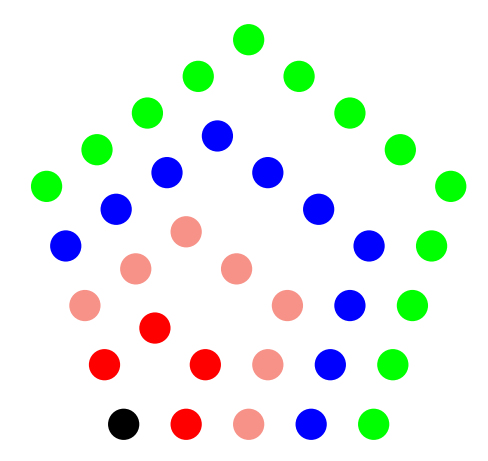

\section{Palindromic pentagonal numbers}

\begin{tabular}{|cc||cc||cc|}
\hline$n$ & $P_{n}$ & $n$ & $P_{n}$ & $n$ & $P_{n}$ \\
\hline \hline 1 & 1 & 101 & 12521 & 6010 & 54177145 \\
2 & 5 & 693 & 720027 & 26466 & 1050660501 \\
4 & 22 & 2173 & 7081807 & 26906 & 1085885801 \\
26 & 1001 & 2229 & 7451547 & 31926 & 1528888251 \\
44 & 2882 & 4228 & 26811862 & 44059 & 2911771192 \\
\hline
\end{tabular}




\subsection{The polygonal numbers $P_{n, k}$}

More generally, for a fixed $k$, the $k$-gonal numbers are the sums of the arithmetic progression

$$
1+(k-1)+(2 k-3)+\cdots .
$$

The $n$th $k$-gonal number is $P_{k, n}=\frac{1}{2} n((k-2) n-(k-4))$.

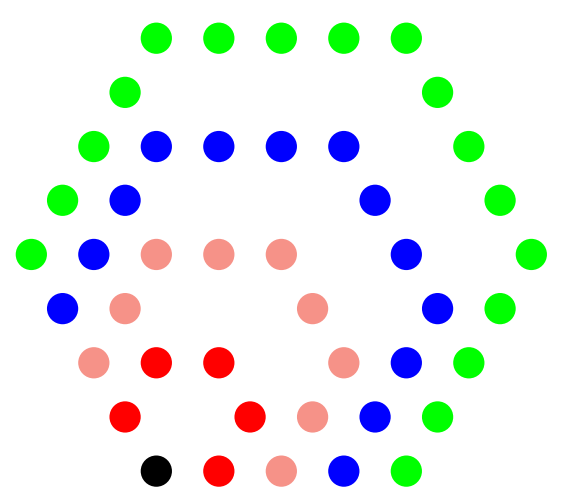




\subsubsection{Appendix: Solution of Pell's equation}

(1) Let $d$ be a positive integer which is not a square. The positive integer solutions of the equation $x^{2}-d y^{2}=1$ can be arranged in a sequence as follows. If $(x, y)=(a, b)$ is the smallest positive solution, then

$$
\left(\begin{array}{l}
x_{n+1} \\
y_{n+1}
\end{array}\right)=\left(\begin{array}{cc}
a & d b \\
b & a
\end{array}\right)\left(\begin{array}{l}
x_{n} \\
y_{n}
\end{array}\right), \quad\left(\begin{array}{l}
x_{1} \\
y_{1}
\end{array}\right)=\left(\begin{array}{l}
a \\
b
\end{array}\right) .
$$

(2) If the equation $x^{2}-d y^{2}=-1$ has a solution in nonzero integers, its integer solutions can be arranged in the form a sequence satisfying the same recurrence relation above (with $(a, b)$ the smallest positive solution of $\left.x^{2}-d y^{2}=1\right)$ but with $\left(x_{1}, y_{1}\right)$ given by its smallest positive solution. 


\section{Exercise}

1. Prove that every hexagonal number is a triangular number.

2. Find two triangular numbers (apart from 1) that are squares.

3. Find a pentagonal numbers (apart from 1) that is also a square.

4. It is known that there is only one value of $n$ for which

$$
1^{2}+2^{2}+3^{2}+\cdots+n^{2}
$$

is a square. What is this value?

5. Ramanujan's house number.

6. An unidentified country has 7-digit population - and everyone has been given a National ID Number, sequentially from one, allocated by no identifiable logic.

The Censure Minister has chosen three names at random, and is finding their ID number on the computer. When the first number appears on the screen, the Government's mathematical whiz-kid informs the Minister that there is precisely a 50-50 chance that the other two numbers will both be less than the one just displayed.

What is the population, and what is the first number? ${ }^{1}$

\footnotetext{
${ }^{1}$ Problem 2585, JRM, 31 (2002-2003) 71.
} 


\section{Chapter 28}

\section{Polygonal triples}

We consider polygonal numbers of a fixed shape. For a given positive integer $k$, the sequence of $k$-gonal numbers consists of the integers

$$
P_{k, n}:=\frac{1}{2}\left((k-2) n^{2}-(k-4) n\right) .
$$

By a $k$-gonal triple, we mean a triple of positive integers $(a, b, c)$ satisfying

$$
P_{k, a}+P_{k, b}=P_{k, c} .
$$

A 4-gonal triple is simply a Pythagorean triple satisfying $a^{2}+b^{2}=c^{2}$. We shall assume that $k \neq 4$. By completing squares, we rewrite (28.2) as

$$
\begin{aligned}
& (2(k-2) a-(k-4))^{2}+(2(k-2) b-(k-4))^{2} \\
= & (2(k-2) c-(k-4))^{2}+(k-4)^{2}
\end{aligned}
$$

and note, by dividing throughout by $(k-4)^{2}$, that this determines a rational point on the surface $\mathcal{S}$ :

$$
x^{2}+y^{2}=z^{2}+1
$$

namely,

$$
P(k ; a, b, c):=(g a-1, g b-1, g c-1),
$$

where $g=\frac{2(k-2)}{k-4}$. This is always an integer point for $k=3,5,6,8$, with corresponding $g=-2,6,4,3$. For $k=3$ (triangular numbers), we shall change signs, and consider instead the point

$$
P^{\prime}(3 ; a, b, c):=(2 a+1,2 b+1,2 c+1) .
$$

The coordinates of $P^{\prime}(3 ; a, b, c)$ are all odd integers exceeding 1 . 


\subsection{Double ruling of $\mathcal{S}$}

The surface $\mathcal{S}$, being the surface of revolution of a rectangular hyperbola about its conjugate axis, is a rectangular hyperboloid of one sheet. It has a double ruling, i.e., through each point on the surface, there are two straight lines lying entirely on the surface.

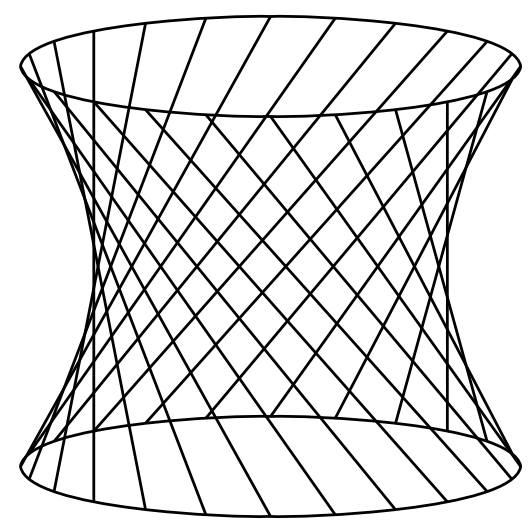

Figure 28.1:

Let $P\left(x_{0}, y_{0}, z_{0}\right)$ be a point on the surface $\mathcal{S}$. A line $\ell$ through $P$ with direction numbers $p: q: r$ has parametrization

$$
\ell: \quad x=x_{0}+p t, \quad y=y_{0}+q t, \quad z=z_{0}+r t .
$$

Substitution of these expressions into (28.4) shows that the line $\ell$ is entirely contained in the surface $\mathcal{S}$ if and only if

$$
\begin{aligned}
p x_{0}+q y_{0} & =r z_{0}, \\
p^{2}+q^{2} & =r^{2} .
\end{aligned}
$$

It follows that

$$
\begin{aligned}
r^{2} & =r^{2}\left(x_{0}^{2}+y_{0}^{2}-z_{0}^{2}\right) \\
& =r^{2}\left(x_{0}^{2}+y_{0}^{2}\right)-\left(p x_{0}+q y_{0}\right)^{2} \\
& =\left(p^{2}+q^{2}\right)\left(x_{0}^{2}+y_{0}^{2}\right)-\left(p x_{0}+q y_{0}\right)^{2} \\
& =\left(q x_{0}-p y_{0}\right)^{2} .
\end{aligned}
$$

This means

$$
q x_{0}-p y_{0}=\epsilon r, \quad \epsilon= \pm 1 .
$$

Solving equations (28.7) and (28.9), we determine the direction numbers of the line. We summarize this in the following proposition. 
Proposition 28.1. The two lines lying entirely on the hyperboloid $\mathcal{S}$ : $x^{2}+y^{2}=z^{2}+1$ and passing through $P\left(x_{0}, y_{0}, z_{0}\right)$ have direction numbers

$$
x_{0} z_{0}-\epsilon y_{0}: y_{0} z_{0}+\epsilon x_{0}: x_{0}^{2}+y_{0}^{2}
$$

for $\epsilon= \pm 1$.

In particular, if $P$ is a rational point, these direction numbers are rational.

\subsection{Primitive Pythagorean triple associated with a $k$ - gonal triple}

Let $P$ be the rational point determined by a $k$-gonal triple $(a, b, c)$, as given by (28.5), for $k \geq 5$ and (28.6) for $k=3$ (triangular numbers). We first note that the coordinates of $P$ all exceed 1 . This is clear for $k=3$, and for $k \geq 5$, it follows from the fact that $g=\frac{2(k-2)}{k-4}>2$. The direction numbers of the ruling lines on $\mathcal{S}$ through the point $P$, as given in Proposition 1, are all positive. In view of (28.8), we may therefore choose a primitive Pythagorean triple $(p, q, r)$ for these direction numbers. As is well known, every such triple is given by

$$
p=m^{2}-n^{2}, \quad q=2 m n, \quad r=m^{2}+n^{2}
$$

for relatively prime integers $m>n$ of different parity.

We study the converse question of determining $k$-gonal triples from (primitive) Pythagorean triples.

\subsection{Triples of triangular numbers}

Given a primitive Pythagorean triple $(p, q, r)$ as in (28.10), we want to determine a triangular triple $(a, b, c)$ corresponding to it. Given an odd integer $z_{0}>1$, we obtain, from (28.7) and (28.9),

$$
x_{0}=\frac{p z_{0}+\epsilon q}{r}, \quad y_{0}=\frac{q z_{0}-\epsilon p}{r} .
$$

We claim that it is possible to choose $z_{0}>1$ so that $x_{0}$ and $y_{0}$ are also odd integers $>1$.

By the euclidean algorithm, there are odd integers $u$ and $v$ such that $q u+r v=1$. (Note that $v$ must be odd, since $q$ is even. If $u$ is even, we 
replace $(u, v)$ by $(u-r, v+q)$, in which both entries are odd). Clearly, the integer $z_{0}=\epsilon p u$ is such that $q z_{0}-\epsilon p=\epsilon p(q u-1)$ is divisible by $r$. This makes $y_{0}$ an integer. The corresponding $x_{0}$ is also an integer. Replacing $z_{0}$ by $z_{0}+r t$ for a positive integer $t$ if necessary, the integers $z_{0}, x_{0}$, and $y_{0}$ can be chosen greater than 1 . From (28.11), the integers $x_{0}$ and $y_{0}$ are both odd, since $p$ and $q$ are of different parity and $z_{0}$ is odd.

We summarize this in the following theorem.

Theorem 28.2. Let $(p, q, r)$ be a primitive Pythagorean triple. There are two infinite families of triangular triples $\left(a_{\epsilon}(t), b_{\epsilon}(t), c_{\epsilon}(t)\right), \epsilon= \pm 1$, such that one of the lines $\ell_{\epsilon}(P), P=P^{\prime}\left(3 ; a_{\epsilon}(t), b_{\epsilon}(t), c_{\epsilon}(t)\right)$, has direction numbers $p: q: r$.

\section{Triangular triples from primitive Pythagorean triples}

\begin{tabular}{|c|c|c|c|}
\hline$(m, n)$ & $(p, q, r)$ & $\left(a_{+}(0), b_{+}(0), c_{+}(0)\right)$ & $\left(a_{-}(0), b_{-}(0), c_{-}(0)\right)$ \\
\hline \hline$(2,1)$ & $(3,4,5)$ & $(2,2,3)$ & $(3,5,6)$ \\
\hline$(4,1)$ & $(15,8,17)$ & $(9,4,10)$ & $(5,3,6)$ \\
\hline$(3,2)$ & $(5,12,13)$ & $(4,9,10)$ & $(5,14,15)$ \\
\hline$(6,1)$ & $(35,12,37)$ & $(20,6,21)$ & $(14,5,15)$ \\
\hline$(5,2)$ & $(21,20,29)$ & $(6,5,8)$ & $(14,14,20)$ \\
\hline$(4,3)$ & $(7,24,25)$ & $(6,20,21)$ & $(7,27,28)$ \\
\hline$(8,1)$ & $(63,16,65)$ & $(35,8,36)$ & $(27,7,28)$ \\
\hline$(7,2)$ & $(45,28,53)$ & $(35,21,41)$ & $(9,6,11)$ \\
\hline$(5,4)$ & $(9,40,41)$ & $(8,35,36)$ & $(9,44,45)$ \\
\hline
\end{tabular}

\section{$28.4 k$-gonal triples determined by a Pythagorean triple}

Now, we consider $k \geq 5$. We shall adopt the notation

$$
h^{\prime}:= \begin{cases}h & \text { if } h \text { is odd } \\ \frac{h}{2} & \text { if } h \text { is even }\end{cases}
$$

for an integer $h$.

Theorem 28.3. Let $k \geq 5$ and $g=\frac{2(k-4)}{k-2}$. The primitive Pythagorean triple $(p, q, r)$ defined in (28.10) by relatively prime integers $m>n$ with different parity corresponds to a $k$-gonal triple if and only if one of $\frac{2 n}{g}$ and $\frac{2(m-n)}{g}$ is an integer.

Since $m$ and $n$ are relatively prime, the integer $(k-2)^{\prime}>1$ cannot divide both $n$ and $m-n$. This means that a primitive Pythagorean triple 
$(p, q, r)$ corresponds to at most one line on $\mathcal{S}$ associated with $k$-gonal triples (for $k \geq 5$ ).

Indeed, if $k=4 h+2,(k-2)^{\prime}$ is the even number $2 h$, and cannot divide the odd integer $m-n$. It follows that only those pairs $(m, n)$, with $n$ a multiple of $2 h$ give $(4 h+2)$-gonal pairs. For example, by choosing $m=2 h+1, n=2 h$, we have

$$
\begin{array}{lll}
p=4 h+1, & q=8 h^{2}+4 h, & r=8 h^{2}+4 h+1 \\
a_{0}=4 h+1, & b_{0}=8 h^{2}+2 h+1, & c_{0}=8 h^{2}+2 h+2 .
\end{array}
$$

These give an infinite family of $(4 h+2)$-gonal triples:

$$
\begin{aligned}
a_{t} & =(4 h+1)(t+1) \\
b_{t} & =8 h^{2}+2 h+1+\left(8 h^{2}+4 h\right) t \\
c_{t} & =8 h^{2}+2 h+2+\left(8 h^{2}+4 h+1\right) t
\end{aligned}
$$

$$
\underline{(4 h+2)-\text { gonal triples }}
$$

\begin{tabular}{|c|c|c|c|}
\hline$(h, k, g)$ & $(m, n)$ & $(p, q, r)$ & $(a, b, c)$ \\
\hline \hline$(1,6,4)$ & $(3,2)$ & $(5,12,13)$ & $(5,11,12)$ \\
\hline & $(5,2)$ & $(21,20,29)$ & $(14,13,19)$ \\
\hline & $(5,4)$ & $(9,40,41)$ & $(9,38,39)$ \\
\hline & $(7,2)$ & $(45,28,53)$ & $(18,11,21)$ \\
\hline & $(7,4)$ & $(33,56,65)$ & $(11,18,21)$ \\
\hline & $(7,6)$ & $(13,84,85)$ & $(13,81,82)$ \\
\hline & $(9,2)$ & $(77,36,85)$ & $(11,5,12)$ \\
\hline & $(9,4)$ & $(65,72,97)$ & $(13,14,19)$ \\
\hline & $(9,8)$ & $(17,144,145)$ & $(17,140,141)$ \\
\hline & $(11,2)$ & $(117,44,125)$ & $(104,39,111)$ \\
\hline & $(11,4)$ & $(105,88,137)$ & $(60,50,78)$ \\
\hline & $(11,6)$ & $(85,132,157)$ & $(68,105,125)$ \\
\hline & $(11,8)$ & $(57,176,185)$ & $(38,116,122)$ \\
\hline & $(11,10)$ & $(21,220,221)$ & $(21,215,216)$ \\
\hline$\left(2,10, \frac{8}{3}\right)$ & $(5,4)$ & $(9,40,41)$ & $(9,37,38)$ \\
\hline & $(7,4)$ & $(33,56,65)$ & $(33,55,64)$ \\
\hline & $(9,4)$ & $(65,72,97)$ & $(52,57,77)$ \\
\hline & $(9,8)$ & $(17,144,145)$ & $(17,138,139)$ \\
\hline & $(11,4)$ & $(105,88,137)$ & $(90,75,117)$ \\
\hline \hline$\left(3,14, \frac{12}{5}\right)$ & $(11,8)$ & $(57,176,185)$ & $(57,174,183)$ \\
\hline & $(7,6)$ & $(13,84,85)$ & $(13,79,80)$ \\
\hline & & & $(85,131,156)$ \\
\hline & $(11,6)$ & $(85,132,157)$ & \\
\hline & & & \\
\hline & & & \\
\hline & &
\end{tabular}




\subsection{Correspondence between $(2 h+1)$-gonal and $4 h$ - gonal triples}

Let $k_{1}<k_{2}$ be two positive integers $\geq 5$. Theorem 2 suggests that there is a one-to-one correspondence between $k_{1}$-gonal triples and $k_{2}$-gonal triples, provided $\left(k_{1}-2\right)^{\prime}=\left(k_{2}-2\right)^{\prime}$. This is the case if and only if

$$
k_{1}=2 h+1, \quad k_{2}=4 h, \text { for some } h \geq 2 .
$$

In this case, $\left(k_{1}-2\right)^{\prime}=\left(k_{2}-2\right)^{\prime}=2 h-1$, while $\left(k_{1}-4\right)^{\prime}=2 h-3$, and $\left(k_{2}-4\right)^{\prime}=2 h-2$. The $(2 h+1)$-gonal triple $(a, b, c)$ and a $4 h$-gonal triple $\left(a^{\prime}, b^{\prime}, c^{\prime}\right)$ are related by

$$
\begin{gathered}
(m-n)\left(c-c^{\prime}\right) \equiv \frac{n}{2 h-1} \quad\left(\bmod m^{2}+n^{2}\right) . \\
(2 h+1)-\text { gonal and } 4 h-\text { gonal triples }
\end{gathered}
$$

\begin{tabular}{|c|c|c|c|c|}
\hline$(h, 2 h+1,4 h)$ & $(m, n)$ & $(p, q, r)$ & $\begin{array}{c}(2 h+1)-\text { gonal } \\
(a, b, c)\end{array}$ & $\begin{array}{c}4 h-\text { gonal } \\
\left(a^{\prime}, b^{\prime}, c^{\prime}\right)\end{array}$ \\
\hline \hline$(2,5,8)$ & $(4,1)$ & $(15,8,17)$ & $(7,4,8)$ & $(14,8,16)$ \\
\hline & $(4,3)$ & $(7,24,25)$ & $(7,23,24)$ & $(7,22,23)$ \\
\hline & $(5,2)$ & $(21,20,29)$ & $(5,5,7)$ & $(10,10,14)$ \\
\hline & $(7,4)$ & $(33,56,65)$ & $(4,7,8)$ & $(8,14,16)$ \\
\hline & $(7,6)$ & $(13,84,85)$ & $(13,82,83)$ & $(13,80,81)$ \\
\hline & $(8,3)$ & $(55,48,73)$ & $(22,19,29)$ & $(44,38,58)$ \\
\hline & $(8,5)$ & $(39,80,89)$ & $(35,72,80)$ & $(31,64,71)$ \\
\hline & $(10,1)$ & $(99,20,101)$ & $(48,10,49)$ & $(96,20,98)$ \\
\hline & $(10,3)$ & $(91,60,109)$ & $(26,17,31)$ & $(52,34,62)$ \\
\hline & $(10,7)$ & $(51,140,149)$ & $(40,110,117)$ & $(29,80,85)$ \\
\hline & $(10,9)$ & $(19,180,181)$ & $(19,177,178)$ & $(19,174,175)$ \\
\hline & $(6,1)$ & $(35,12,37)$ & $(16,6,17)$ & $(33,12,35)$ \\
\hline & $(6,5)$ & $(11,60,61)$ & $(11,57,58)$ & $(11,56,57)$ \\
\hline & $(7,2)$ & $(45,28,53)$ & $(33,21,39)$ & $(44,28,52)$ \\
\hline & $(8,3)$ & $(55,48,73)$ & $(27,24,36)$ & $(36,32,48)$ \\
\hline & $(8,5)$ & $(39,80,89)$ & $(39,79,88)$ & $(26,52,58)$ \\
\hline & $(9,4)$ & $(65,72,97)$ & $(24,27,36)$ & $(32,36,48)$ \\
\hline$(4,9,16)$ & $(8,1)$ & $(63,16,65)$ & $(29,8,30)$ & $(60,16,62)$ \\
\hline & $(8,7)$ & $(15,112,113)$ & $(15,107,108)$ & $(15,106,107)$ \\
\hline & $(9,2)$ & $(77,36,85)$ & $(18,9,20)$ & $(37,18,41)$ \\
\hline & $(10,3)$ & $(91,60,109)$ & $(75,50,90)$ & $(90,60,108)$ \\
\hline & $(10,7)$ & $(51,140,149)$ & $(17,45,48)$ & $(51,138,147)$ \\
\hline & $(10,1)$ & $(99,20,101)$ & $(46,10,47)$ & $(95,20,97)$ \\
\hline & $(10,9)$ & $(19,180,181)$ & $(19,173,174)$ & $(19,172,173)$ \\
\hline & & & & \\
\hline & & & \\
\hline & & & & \\
\hline & & & & \\
\hline & & &
\end{tabular}




\section{Chapter 29}

\section{Sums of consecutive squares}

1 Sum of squares of natural numbers

2 Sums of consecutive squares: odd number case

3 Sums of consecutive squares: even number case 


\subsection{Sum of squares of natural numbers}

\section{Theorem 29.1.}

$$
1^{2}+2^{2}+3^{2}+\cdots+n^{2}=\frac{1}{6} n(n+1)(2 n+1) .
$$

Proof. Let $T_{n}=1+2+3 \cdots+n=\frac{1}{2} n(n+1)$ and

$$
\begin{aligned}
& S_{n}=1^{2}+2^{2}+3^{2}+\cdots+n^{2} . \\
& 2^{3}=1^{3}+3 \cdot 1^{2}+3 \cdot 1+1 \\
& 3^{3}=2^{3}+3 \cdot 2^{2}+3 \cdot 2+1 \\
& 4^{3}=3^{3}+3 \cdot 3^{2}+3 \cdot 3+1 \\
& n^{3}=(n-1)^{3}+3(n-1)^{2}+3(n-1)+1 \\
& (n+1)^{3}=n^{3}+3 \cdot n^{2}+3 \cdot n+1
\end{aligned}
$$

Combining these equations, we have

$$
(n+1)^{3}=1^{3}+3 S_{n}+3 T_{n}+n .
$$

Since $T_{n}$ is known, we have

$$
S_{n}=\frac{1}{3}\left((n+1)^{3}-n-1-\frac{3}{2} n(n+1)\right)=\frac{1}{6} n(n+1)(2 n+1) .
$$

\section{Exercise}

1. Find $1^{2}+3^{2}+5^{2}+\cdots+(2 n-1)^{2}$.

2. Find $n$ so that $n^{2}+(n+1)^{2}$ is a square. 


\subsection{Sums of consecutive squares: odd number case}

Suppose the sum of the squares of $2 k+1$ consecutive positive integers is a square. If the integers are $b, b \pm 1, \ldots, b \pm k$. We require

$$
(2 k+1) b^{2}+\frac{1}{3} k(k+1)(2 k+1)=a^{2}
$$

for an integer $a$. From this we obtain the equation

$$
a^{2}-(2 k+1) b^{2}=\frac{1}{3} k(k+1)(2 k+1) .
$$

1. Suppose $2 k+1$ is a square. Show that $\left(E_{k}\right)$ has solution only when $k=6 m(m+\epsilon)$ for some integers $m>1$, and $\epsilon= \pm 1$. In each case, the number of solutions is finite.

\section{Number of solutions of $\left(E_{k}\right)$ when $2 k+1$ is a square}

$$
\begin{array}{cccccccccccc}
2 k+1 & 25 & 49 & 121 & 169 & 289 & 361 & 529 & 625 & 841 & 961 & \ldots \\
& 0 & 1 & 1 & 2 & 7 & 3 & 5 & 3 & 3 & 10 & \ldots
\end{array}
$$

2. Find the unique sequence of 49 (respectively 121) consecutive positive integers whose squares sum to a square.

3. Find the two sequences of 169 consecutive squares whose sums are squares.

4. Suppose $2 k+1$ is not a square. If $k+1$ is divisible $9=3^{2}$ or by any prime of the form $4 k+3 \geq 7$, then the equation $\left(E_{k}\right)$ has no solution. Verify that for the following values of $k<50$, the equation $\left(E_{k}\right)$ has no solution: 


$$
\begin{aligned}
k= & 6,8,10,13,17,18,20,21,22,26,27,30,32, \\
& 34,35,37,40,41,42,44,45,46,48, \ldots
\end{aligned}
$$

5. Suppose $p=2 k+1$ is a prime. If the Legendre symbol $\left(\frac{-\frac{1}{3} k(k+1)}{p}\right)=$ -1 , then the equation $\left(E_{k}\right)$ has no solution. Verify that for the following values of $k<50$, the equation $\left(E_{k}\right)$ has no solution:

$$
1,2,3,8,9,14,15,20,21,26,33,39,44 .
$$

6. For $k \leq 50$, it remains to consider $\left(E_{k}\right)$ for the following values of $k$ :

$$
5,7,11,16,19,23,25,28,29,31,36,38,43,47,49 .
$$

Among these, only for $k=5,11,16,23,29$ are the equations $\left(E_{k}\right)$ solvable.

7. Work out 5 sequences of 23 consecutive integers whose squares add up to a square in each case.

Answer:

$$
\begin{aligned}
7^{2}+8^{2}+\cdots+29^{2} & =92^{2} \\
881^{2}+882^{2}+\cdots+903^{2} & =4278^{2} ; \\
42787^{2}+42788^{2}+\cdots+42809^{2} & =205252^{2} ; \\
2053401^{2}+2053402^{2}+\cdots+2053423^{2} & =9847818^{2}
\end{aligned}
$$

8. Consider the equation $\left(E_{36}\right): u^{2}-73 v^{2}=12 \cdot 37 \cdot 73$. This equation does in fact have solutions $(u, v)=(4088,478),(23360,2734)$. The fundamental solution of the Pell equation $x^{2}-73 y^{2}=1$ being $(a, b)=(2281249,267000)$, we obtain two sequences of solutions of $\left(E_{73}\right)$ :

Answer:

$(4088,478),(18642443912,2181933022),(85056113063608088,9955065049008478), \ldots$ $(23360,2734),(106578370640,12474054766),(486263602888235360,56912849921762734), \ldots$

This means, for example, the sum of the squares of the 73 numbers with center 478 (respectively 2734) is equal to the square of 4088 (respectively 23360). 


\subsection{Sums of consecutive squares: even number case}

Suppose the sum of the squares of the $2 k$ consecutive numbers

$$
b-k+1, b-k+2, \ldots, b, \ldots, b+k-1, b+k,
$$

is equal to $a^{2}$. This means

$$
(2 a)^{2}-2 k(2 b+1)^{2}=\frac{2 k}{3}\left(4 k^{2}-1\right) .
$$

Note that the numbers $2 k, 4 k^{2}-1$ are relatively prime.

1. Show that the equation $\left(E_{k}^{\prime}\right)$ has no solution if $2 k$ is a square.

2. Suppose $2 k$ is not a square. Show that if $2 k+1$ is divisible by 9 , or by any prime of the form $4 k+1$, then the equation $\left(E_{k}^{\prime}\right)$ has no solution.

3. For $k \leq 50$, the equation $\left(E_{k}^{\prime}\right)$ has no solution for the following values of $k$ :

$$
\begin{aligned}
k= & 3,4,5,9,11,13,15,17,21,23,24,27,29,31,33, \\
& 35,38,39,40,41,45,47,49 .
\end{aligned}
$$

4. Let $k$ be a prime. The equation $\left(E_{k}^{\prime}\right)$ can be written as

$$
(2 b+1)^{2}-2 k y^{2}=-\frac{4 k^{2}-1}{3} .
$$

By considering Legendre symbols, the equation $\left(E_{k}^{\prime}\right)$ has no solution for the following values of $k \leq 50$ :

$$
k=5,7,17,19,29,31,41,43 .
$$

5. Excluding square values of $2 k<100$, the equation $\left(E_{k}^{\prime}\right)$ has solutions only for $k=1,12,37,44$.

6. Show that $(34,0),(38,3),(50,7)$ are solutions of $\left(E "{ }_{12}\right)$. Construct from them three infinite sequences of expressions of the sum of 24 consecutive squares as a square. 
7. The equation $\left(E_{37}^{\prime}\right)$ has solutions $(185,2),(2257,261)$, and $(2849$, $330)$. From these we construct three infinite sequences of expressions of the sum of 74 consecutive squares as a square.

Answer:

$$
\begin{aligned}
225^{2}+226^{2}+\cdots+298^{2} & =2257^{2} \\
294^{2}+295^{2}+\cdots+367^{2} & =2849^{2} \\
13096^{2}+13097^{2}+\cdots+13179^{2} & =763865^{2} .
\end{aligned}
$$

8. The equation $\left(E_{44}^{\prime}\right)$ has solutions $(242,4)$ and $(2222,235)$. From these we obtain two infinite sequences of expressions of the sum of 88 consecutive squares as a square.

$$
\begin{aligned}
192^{2}+193^{2}+\cdots+279^{2} & =2222^{2} \\
5925^{2}+5926^{2}+\cdots 6012^{2} & =55990^{2} .
\end{aligned}
$$




\section{Chapter 30}

\section{Sums of powers of natural numbers}

Notation

$$
S_{k}(n):=1^{k}+2^{k}+\cdots+n^{k} .
$$

Theorem 30.1 (Bernoulli). $S_{k}(n)$ is a polynomial in $n$ of degree $k+1$ without constant term. It can be obtained recursively as

$$
S_{k+1}(n)=\int(k+1) S_{k}(n) d n+c n
$$

where $c$ is determined by the condition that the sum of the coefficients is 1.

\section{Examples}

(1) $S_{3}(n)=1^{3}+2^{3}+\cdots+n^{3}=\frac{1}{4} n^{2}(n+1)^{2}$.

(2) Since $4 S_{3}(n)=n^{4}+2 n^{3}+n^{2}$, we have

$$
S_{4}(n)=\frac{1}{5} n^{5}+\frac{1}{2} n^{4}+\frac{1}{3} n^{3}+c n,
$$

where $c=1-\left(\frac{1}{5}+\frac{1}{2}+\frac{1}{3}\right)=\frac{-1}{30}$. Therefore,

$$
1^{4}+2^{4}+\cdots+n^{4}=\frac{1}{5} n^{5}+\frac{1}{2} n^{4}+\frac{1}{3} n^{3}-\frac{1}{30} n .
$$




\section{Exercise}

1. Find the sum of the first $n$ odd numbers.

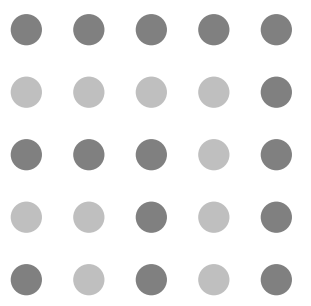

2. Find the sum of the cubes of the first $n$ odd numbers.

3. Find $S_{5}(n)$ and $S_{6}(n)$.

4. Find the sum of the series

$$
1 \cdot 2 \cdot 3+2 \cdot 3 \cdot 4+3 \cdot 4 \cdot 5+\cdots+n(n+1)(n+2) .
$$

5. Find the sum of the first $n$ triangular numbers. 


\section{Chapter 31}

\section{A high school mathematics contest}

\section{Christopher Newport University Regional High School Mathemat-} ics Contest, November, $2002{ }^{1}$

1. Randy and Hannah are eating at a restaurant. The items ordered by Randy cost twice as much as the items ordered by Hannah. Randy leaves a tip of $15 \%$ of the price of what he has ordered. Hannah leaves a tip of $20 \%$ of her items. The total, including tips, paid by the pair is exactly 70 dollars. How much was the cost of the items Hannah ordered?

\footnotetext{
${ }^{1}$ Crux Math., 29 (2003) 193-195.
} 
2. Solve the equation

$$
x^{2}-|x|-1=0 \text {. }
$$

3. Let $\left(a_{n}\right)$ be an arithmetic sequence. If $a_{p}=q$ and $a_{q}=p$, find $a_{p+q}$.

4. A five-digit number is called a mountain number if the first three digits are increasing and the last three are decreasing. For example, 34541 is a mountain number, but 34534 is not. How many mountain numbers are greater than 70000 ? 
5. Each day, Hai and Wai separately play a video game and compare scores. Hai's score on Tuesday was $10 \%$ less than his score on Monday, while Wai's score on Tuesday was 20 points higher than on Monday. However, on Wednesday, Hai's score was 10 points higher than on Tuesday, while Wai's score on Wednesday was 20\%

less than his score on Tuesday. Strangely, Hai's score plus Wai's score turned out to be the same on all three days. What were their scores on Wednesday? 
6. A point $P$ is given in the interior of a rectangle $A B C D$ with $A B=$ $C D=24$ and $A D=B D=5$. What is the total area of the two triangles $P A D$ and $P B C$ (shaded in the figure)?

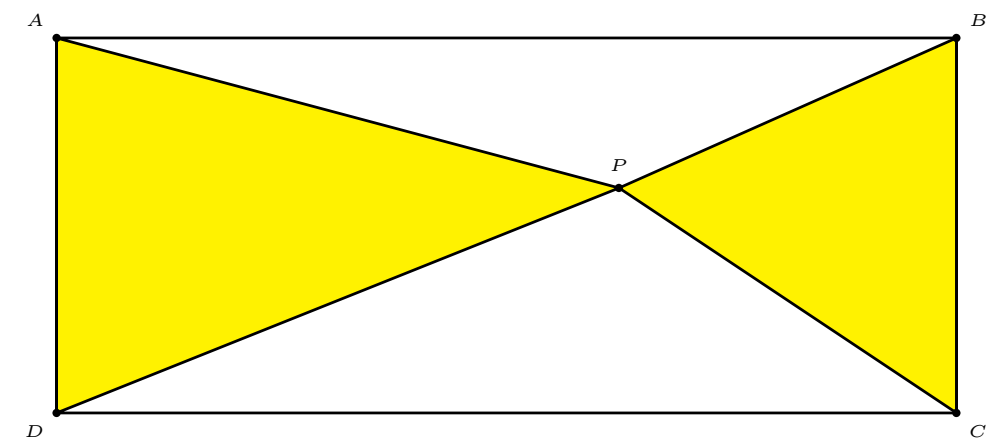

7. Samantha bought a stock for 1000 dollars whose price then doubled every year for the next $n$ years. In the year after that, the stock price fell by $99 \%$. Nevertheless, the stock was still worth than 1000 dollars. What is the smallest whole number of years for which this is possible? 
8. In triangle $A B C, \cos (A-B)+\sin (A+B)=2$. Determine the shape of the triangle.

9. Four small circles of radius 1 are tangent to each other and to a larger circle containing them, as shown in the diagram. What is the area of the region inside the larger circle, but outside all the smaller circles?

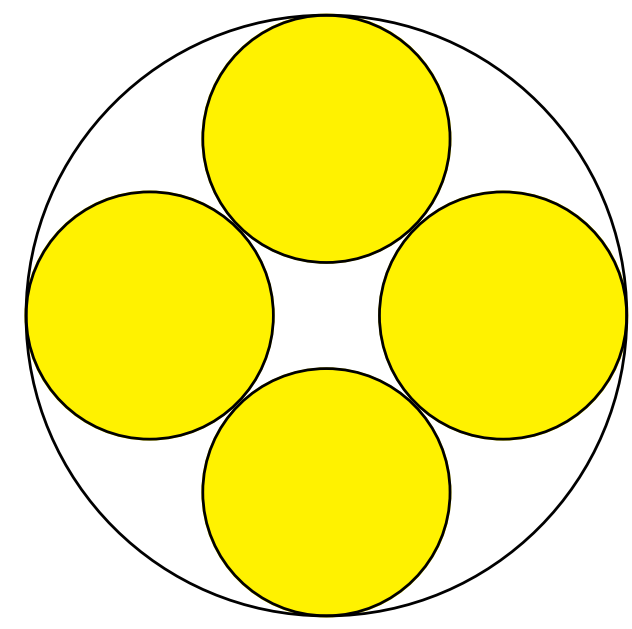


10. Two circles of radii 9 and 17 centimeters are enclosed in a rectangle with one side of length 50 centimeters. The two circles touch each other, and each touches two adjacent sides of the rectangle, as indicated. Find the area of the rectangle.

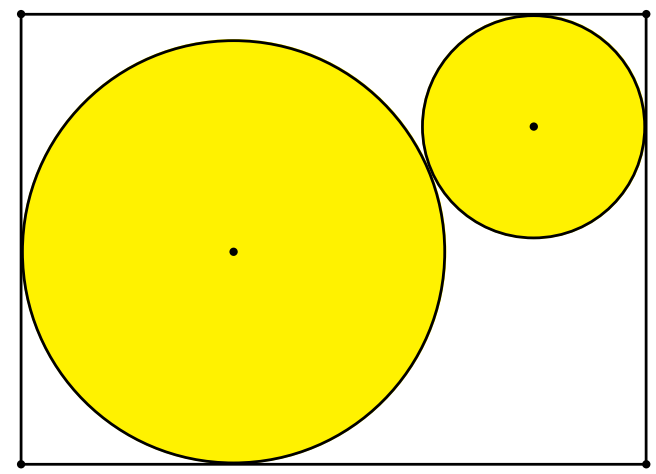


11. Find three different prime numbers $a, b, c$ so that their sum $a+b+c$ and their product $a b c$ both end in the digit 7 .

12. Karen ran a 42 kilometer marathon in 3 hours, 49 minutes. She did this by running for 10 minutes, walking for 2 minutes, then running for 10 minutes, walking for 2 minutes, and so on until she crossed the finish line. She runs twice as fast as she walks. What is her average speed, in kilometers per hour, while running? 


\section{Chapter 32}

\section{Mathematical entertainments}

1 David Wells' survey of Beauty in Mathematics

2 T. M. Apostol's mathematical acrostic 


\subsection{Beauty in mathematics: David Wells' survey ${ }^{1}$}

Give each of the following theorems a score for beauty between 0 (the least) and 10 (the most beautiful).

\begin{tabular}{|l|l||c|l||c|l||c|l||c|c|}
\hline$A$ & & $B$ & & $C$ & & $D$ & & $E$ & \\
\hline$F$ & & $G$ & & $H$ & & $I$ & & $J$ & \\
\hline$K$ & & $L$ & & $M$ & & $N$ & & $O$ & \\
\hline$P$ & & $Q$ & & $R$ & & $S$ & & $T$ & \\
\hline$U$ & & $V$ & & $W$ & & $X$ & & & \\
\hline
\end{tabular}

A Euler's formula for a polyhedron: $V-E+F=2$.

B Any square matrix satisfies its own characteristic equation.

C If $p(n)$ is the number of partitions of $n$, then

$$
\begin{aligned}
& \frac{5\left(\left(1-x^{2}\right)\left(1-x^{10}\right)\left(1-x^{15}\right) \cdots\right)^{5}}{\left((1-x)\left(1-x^{2}\right)\left(1-x^{3}\right)\left(1-x^{4}\right) \cdots\right)^{6}} \\
= & p(4)+p(9) x+p(14) x^{2}+\cdots
\end{aligned}
$$

D The number of primes is infinite.

$\mathbf{E}$ There is no rational number whose square is 2 .

F Every prime of the form $4 n+1$ is the sum of two integral squares in exactly one way.

G $1+\frac{1}{2^{2}}+\frac{1}{3^{2}}+\cdots+\frac{1}{n^{2}}+\cdots=\frac{\pi^{2}}{6}$.

H $\frac{1}{2 \times 3 \times 4}-\frac{1}{4 \times 5 \times 6}+\frac{1}{6 \times 7 \times 8}-\cdots=\frac{\pi-3}{4}$.

I $\pi$ is transcendental.

$\mathbf{J}$ Every number greater than 77 is the sum of integers, the sum of whose reciprocal is 1 .

$\mathbf{K}$ The maximum area of a quadrilateral with sides $a, b, c, d$ is

$$
\sqrt{(s-a)(s-b)(s-c)(s-d)}
$$

where $s$ is half the perimeter.

\footnotetext{
${ }^{1}$ Math. Intelligencer, 10:4 (1988) 31.
} 
$\mathbf{L}$ There is no equilateral triangle whose vertices are plane lattice points.

M At any party, there is a pair of people who have the same number of friends present.

$\mathbf{N}$ The number of partitions of an integer into odd integers is equal to the number of partitions into distinct integers.

$\mathbf{O}$ If the points of the plane are each colored red, yellow, or blue, there is a pair of points of the same color of mutual distance unity.

P Every plane map can be colored with 4 colors.

Q A continuous mapping of the closed unit disk into itself has a fixed point.

$\mathbf{R}$ Write down the multiples of $\sqrt{2}$, igonoring fractional parts, and underneath the number missing from the first sequence:

$\begin{array}{cccccccccc}1 & 2 & 4 & 5 & 7 & 8 & 9 & 11 & 12 & \ldots \\ 3 & 6 & 10 & 13 & 17 & 20 & 23 & 27 & 30 & \ldots\end{array}$

The difference is $2 n$ in the $n$-th place.

S A regular icosahedron inscribed in a regular octahedron divides the edges in the golden ratio.

$\mathbf{T}$ The number of representations of an odd number as the sum of 4 squares is 8 times the sum of its divisors; of an even number, 24 times the sum of its odd divisors.

$\mathbf{U}$ The word problem for groups is unsolvable.

V The order of a subgroup divides the order the group.

$\mathbf{W} e^{i \pi}=-1$.

$\mathbf{X}$ There are 5 regular polyhedra. 


\subsection{T. M. Apostol's mathematical acrostic ${ }^{2}$}

Guess as many WORDS as you can, then write each letter in the correspondingly numbered square in the diagram. When completely filled in, the diagram contains a passage from a published work concerning mathematics. The initial letters of the WORDS spell out the author and title of the work. All the WORDS are related to mathematics or mathematicians.

\footnotetext{
${ }^{2}$ Math. Intelligencer, 10:3 (1988) 43.
} 


\begin{tabular}{|c|c|c|c|c|c|c|c|c|c|c|c|}
\hline A & Unit of speed & $\overline{131}$ & $\overline{9}$ & $\overline{153}$ & $\overline{62}$ & & & & & & \\
\hline B & $\begin{array}{l}\text { The second cervical } \\
\text { vertebra }\end{array}$ & $\overline{87}$ & $\overline{4}$ & $\overline{177}$ & $\overline{20}$ & & & & & & \\
\hline $\mathrm{C}$ & In opposition & $\overline{160}$ & $\overline{2}$ & $\overline{84}$ & $\overline{28}$ & $\overline{145}$ & $\overline{171}$ & $\overline{104}$ & & & \\
\hline $\bar{D}$ & Countless & $\overline{61}$ & $\overline{49}$ & $\overline{110}$ & $\overline{93}$ & $\overline{63}$ & $\overline{163}$ & $\overline{42}$ & $\overline{183}$ & $\overline{115}$ & $\overline{24}$ \\
\hline $\bar{E}$ & Pallet of an escapement & $\overline{180}$ & $\overline{3}$ & $\overline{137}$ & $\overline{90}$ & $\overline{170}$ & & & & & \\
\hline F & $\begin{array}{l}\text { Successive volumes } \\
\text { (2 words) }\end{array}$ & $\overline{30}$ & $\overline{159}$ & $\overline{41}$ & $\overline{86}$ & $\overline{119}$ & $\overline{75}$ & $\overline{185}$ & & & \\
\hline $\mathrm{G}$ & $\begin{array}{l}\text { Pupil of Gauss, last name } \\
\text { followed by initials }\end{array}$ & $\overline{184}$ & $\overline{45}$ & $\overline{155}$ & $\overline{27}$ & $\overline{125}$ & $\overline{70}$ & $\overline{150}$ & $\overline{43}$ & & \\
\hline $\mathrm{H}$ & $\begin{array}{l}\text { One way to describe } \\
\text { Pythagoras }\end{array}$ & $\overline{15}$ & $\overline{144}$ & $\overline{121}$ & $\overline{82}$ & $\overline{33}$ & $\overline{55}$ & & & & \\
\hline $\mathrm{I}$ & Exact opposite & $\overline{52}$ & $\overline{133}$ & $\overline{12}$ & $\overline{142}$ & $\overline{25}$ & $\overline{101}$ & $\overline{76}$ & $\overline{64}$ & & \\
\hline $\mathrm{J}$ & Make a mosaic of & $\overline{148}$ & $\overline{14}$ & $\overline{21}$ & $\overline{102}$ & $\overline{32}$ & $\overline{141}$ & $\overline{85}$ & $\overline{1}$ & $\overline{182}$ & \\
\hline $\mathrm{K}$ & $\begin{array}{l}\text { First to prove } e \\
\text { transcendental }\end{array}$ & $\overline{114}$ & $\overline{67}$ & $\overline{122}$ & $\overline{10}$ & $\overline{18}$ & $\overline{139}$ & $\overline{158}$ & & & \\
\hline $\mathrm{L}$ & $\begin{array}{l}\text { Logical and sophistical } \\
\text { reasoning }\end{array}$ & $\overline{108}$ & $\overline{31}$ & $\overline{176}$ & $\overline{58}$ & $\overline{100}$ & $\overline{109}$ & $\overline{34}$ & $\overline{111}$ & & \\
\hline $\bar{M}$ & A type of polynomial & $\overline{46}$ & $\overline{81}$ & $\overline{59}$ & $\overline{138}$ & $\overline{149}$ & & & & & \\
\hline$\overline{\mathrm{N}}$ & $\begin{array}{l}\text { Providing pleasure or } \\
\text { delight }\end{array}$ & $\overline{22}$ & $\overline{17}$ & $\overline{35}$ & $\overline{127}$ & $\overline{147}$ & $\overline{29}$ & $\overline{50}$ & $\overline{69}$ & $\overline{97}$ & $\overline{66}$ \\
\hline $\mathrm{O}$ & Added & $\overline{154}$ & $\overline{26}$ & $\overline{7}$ & $\overline{71}$ & $\overline{164}$ & $\overline{53}$ & & & & \\
\hline $\mathrm{P}$ & $\begin{array}{l}\text { Contour connecting points } \\
\text { of equal depth below } \\
\text { a water surface }\end{array}$ & $\overline{5}$ & $\overline{92}$ & $\overline{48}$ & $\overline{60}$ & $\overline{179}$ & $\overline{89}$ & $\overline{146}$ & & & \\
\hline $\bar{Q}$ & $\begin{array}{l}\text { One of the first to use the } \\
\text { method of successive } \\
\text { approximations }\end{array}$ & $\overline{74}$ & $\overline{11}$ & $\overline{124}$ & $\overline{103}$ & $\overline{156}$ & & & & & \\
\hline $\mathrm{R}$ & Equal to the end proposed & $\overline{129}$ & $\overline{169}$ & $\overline{39}$ & $\overline{136}$ & $\overline{132}$ & $\overline{120}$ & 162 & $\overline{72}$ & $\overline{151}$ & $\overline{143}$ \\
\hline $\mathrm{S}$ & Three-digit code & $\overline{98}$ & $\overline{44}$ & $\overline{38}$ & $\overline{130}$ & & & & & & \\
\hline $\mathrm{T}$ & $M^{2}=0$ (two words) & $\overline{80}$ & $\overline{106}$ & $\overline{174}$ & $\overline{168}$ & $\overline{77}$ & $\overline{56}$ & $\overline{91}$ & $\overline{167}$ & $\overline{113}$ & $\overline{116}$ \\
\hline $\mathrm{U}$ & Distribution & $\overline{157}$ & $\overline{23}$ & $\overline{95}$ & $\overline{57}$ & $\overline{79}$ & $\overline{126}$ & $\overline{173}$ & $\overline{88}$ & & \\
\hline $\mathrm{V}$ & $\begin{array}{l}\text { French mathematician } \\
\text { (1765-1843) }\end{array}$ & $\overline{172}$ & $\overline{112}$ & $\overline{19}$ & $\overline{40}$ & $\overline{135}$ & $\overline{96}$ & $\overline{68}$ & & & \\
\hline$\overline{\mathrm{W}}$ & Directed & $\overline{36}$ & $\overline{51}$ & $\overline{134}$ & $\overline{78}$ & $\overline{161}$ & $\overline{123}$ & $\overline{165}$ & & & \\
\hline $\bar{X}$ & Lowness in pitch & $\overline{83}$ & $\overline{65}$ & $\overline{140}$ & $\overline{107}$ & $\overline{54}$ & $\overline{118}$ & $\overline{173}$ & & & \\
\hline$\overline{\mathrm{Y}}$ & $\begin{array}{l}\text { One of Cayley's } \\
\text { hyperdeterminants }\end{array}$ & $\overline{8}$ & $\overline{152}$ & $\overline{105}$ & $\overline{16}$ & $\overline{94}$ & $\overline{166}$ & $\overline{117}$ & $\overline{73}$ & 181 & \\
\hline $\mathrm{Z}$ & $\begin{array}{l}\text { German geometer } \\
(1833-1872)\end{array}$ & $\overline{47}$ & $\overline{99}$ & $\overline{128}$ & $\overline{37}$ & $\overline{6}$ & $\overline{178}$ & $\overline{13}$ & & & \\
\hline
\end{tabular}




\begin{tabular}{|c|c|c|c|c|c|c|c|c|c|c|c|c|}
\hline & $1 \quad J$ & C & & $\begin{array}{ll}3 & E\end{array}$ & $\begin{array}{ll}4 & B\end{array}$ & $\begin{array}{ll}5 & P\end{array}$ & $\begin{array}{ll}6 & Z\end{array}$ & $\begin{array}{ll}7 & 0\end{array}$ & & $8 \quad Y$ & $\begin{array}{ll}9 & A\end{array}$ & \\
\hline $10 \quad K$ & $11 \quad Q$ & $12 \quad I$ & $13 \quad Z$ & $14 \quad J$ & $15 \quad H$ & $16 \quad Y$ & $17 \quad N$ & $18 \quad K$ & $19 \quad V$ & $20 \quad B$ & & 21 \\
\hline$N$ & $23 \quad U$ & $24 \quad D$ & & $25 \quad I$ & $26 \quad 0$ & $27 \quad G$ & $28 \quad C$ & $29 \quad N$ & $\begin{array}{ll}30 & F\end{array}$ & 31 & 32 & \\
\hline$H$ & $34 \quad L$ & & $\begin{array}{ll}35 & N\end{array}$ & $\begin{array}{ll}36 & W\end{array}$ & & $37 \quad Z$ & $38 \quad S$ & & $\begin{array}{ll}39 & R\end{array}$ & $40 \quad V$ & $41 \quad F$ & $42 \quad D$ \\
\hline & $43 \quad G$ & $44 \quad S$ & $45 \quad G$ & $46 \quad M$ & & $47 \quad Z$ & $\begin{array}{ll}48 & P\end{array}$ & $\begin{array}{ll}49 & D\end{array}$ & $\begin{array}{ll}50 & N\end{array}$ & $51 \quad W$ & 52 & $53 \quad 0$ \\
\hline $54 \quad X$ & $\begin{array}{ll}55 & H\end{array}$ & $\begin{array}{ll}56 & T\end{array}$ & $57 \quad U$ & $\begin{array}{ll}58 & L\end{array}$ & $\begin{array}{ll}59 & M\end{array}$ & & $\begin{array}{ll}60 & P\end{array}$ & $\begin{array}{ll}61 & D\end{array}$ & $62 \quad A$ & & $\begin{array}{ll}63 & D\end{array}$ & $64 \quad I$ \\
\hline $65 \quad X$ & $\begin{array}{ll}66 & N \\
\end{array}$ & & $67 \quad K$ & $\begin{array}{ll}68 & V\end{array}$ & $69 \quad N$ & $\begin{array}{ll}70 \quad G \\
\end{array}$ & $\begin{array}{ll}71 & O\end{array}$ & $\begin{array}{ll}72 & R\end{array}$ & $73 \quad Y$ & $\begin{array}{ll}74 & Q\end{array}$ & $\begin{array}{ll}75 \quad F \\
\end{array}$ & \\
\hline $\begin{array}{ll}76 \quad I\end{array}$ & $\begin{array}{ll}77 & T\end{array}$ & $78 \mathrm{~W}$ & $79 \quad U$ & & $\begin{array}{ll}80 & T\end{array}$ & $81 \quad M$ & $82 \quad H$ & & $83 \quad X$ & $84 \quad C$ & 85 & $86 \quad F$ \\
\hline $87 \quad B$ & $88 \quad U$ & $\begin{array}{ll}89 & P\end{array}$ & $\begin{array}{ll}90 & E\end{array}$ & $91 \quad T$ & & $92 \quad P$ & $93 \quad D$ & $94 \quad Y$ & $95 \quad U$ & $96 \quad V$ & $97 \quad N$ & $98 \quad S$ \\
\hline $99 \quad Z$ & & $100 L$ & $101 \quad I$ & & $102 \mathrm{~J}$ & $103 Q$ & $104 \mathrm{C}$ & $105 Y$ & $106 \mathrm{~T}$ & $107 X$ & $108 \mathrm{~L}$ & \\
\hline $109 L$ & $110 \mathrm{D}$ & & $111 L$ & $112 \mathrm{~V}$ & $113 \mathrm{~T}$ & $114 \mathrm{~K}$ & $115 D$ & $116 \mathrm{~T}$ & $117 Y$ & $118 X$ & $119 F$ & $120 R$ \\
\hline $121 \mathrm{H}$ & & $122 \mathrm{~K}$ & $123 \mathrm{~W}$ & $124 Q$ & $125 \mathrm{G}$ & $126 U$ & $127 \mathrm{~N}$ & $128 Z$ & $129 R$ & & $130 \mathrm{~S}$ & \\
\hline $131 A$ & $132 R$ & $133 \quad I$ & $134 \mathrm{~W}$ & & $135 \mathrm{~V}$ & $136 R$ & & $137 E$ & $138 M$ & $139 \mathrm{~K}$ & $140 \mathrm{X}$ & $141 \mathrm{~J}$ \\
\hline $142 I$ & $143 R$ & $144 \mathrm{H}$ & & $145 \mathrm{C}$ & $146 P$ & $147 \mathrm{~N}$ & $148 \mathrm{~J}$ & & $149 M$ & $150 \mathrm{G}$ & $151 R$ & $152 Y$ \\
\hline $153 \mathrm{~A}$ & $154 O$ & & $155 \mathrm{G}$ & $156 Q$ & & $157 U$ & $158 \mathrm{~K}$ & $159 \mathrm{~F}$ & $160 \mathrm{C}$ & $161 \mathrm{~W}$ & $162 R$ & $163 \mathrm{D}$ \\
\hline $164 O$ & $165 \mathrm{~W}$ & & $166 Y$ & $167 \mathrm{~T}$ & & $168 \mathrm{~T}$ & $169 R$ & $170 \mathrm{E}$ & $171 \mathrm{C}$ & $172 \mathrm{~V}$ & $173 X$ & \\
\hline $174 \mathrm{~T}$ & $175 U$ & $176 L$ & $177 B$ & $178 \mathrm{Z}$ & $179 P$ & $180 E$ & & $181 Y$ & $182 \mathrm{~J}$ & $183 D$ & $184 G$ & $185 \mathrm{~F}$ \\
\hline
\end{tabular}




\section{Chapter 33}

\section{Maxima and minima without calculus}

1. We have 1000 feet of fencing and wish to make with it a rectangular pen, at one side of a long wall. How can we arrange to surround the maximum area?

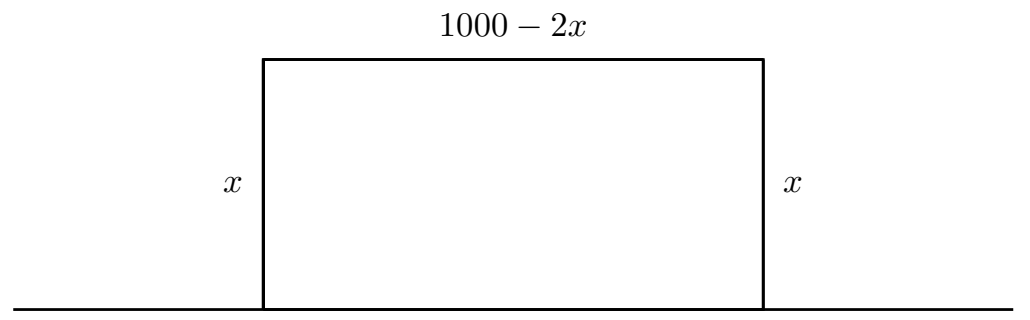


2. A Norman window has a fixed perimeter $a$. Find the largest possible area.

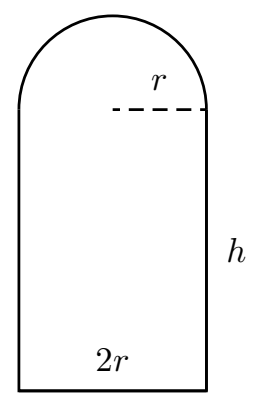

3. A right pyramid has a square base and a given surface area $A$. What is the largest possible volume?

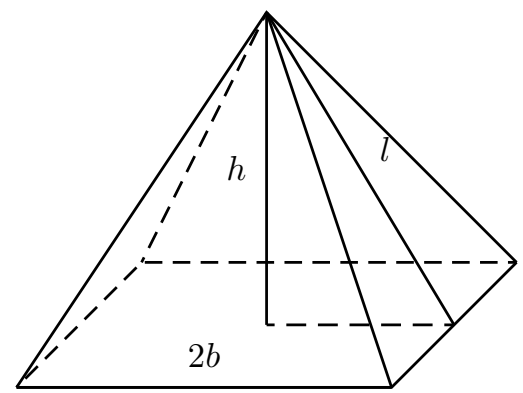


4. A tray is constructed from a square metal sheet by dimension $a \times$ $a$ by cutting squares of the same size from the four corners and folding up the sides. What is the largest possible capacity of the tray?

5. The perimeter of a triangle is a constant $2 s$. What is the largest possible area of the triangle? 
6. The volume of a cylindrical can is given by $V=\pi r^{2} l$ and the surface area by $A=2 \pi r(l+r)$. If the volume is a constant $V$, what is the least possible surface area?

7. Inscribe in a given cone a cylinder whose volume is largest possible.

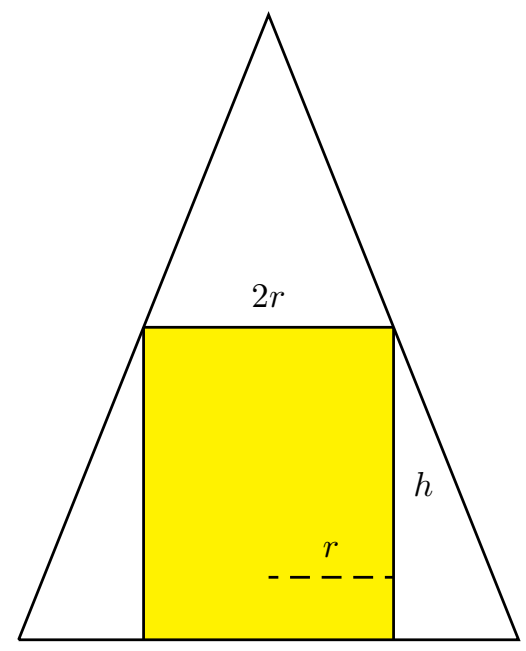


8. Find the largest cylinder contained in a sphere of radius $R$.

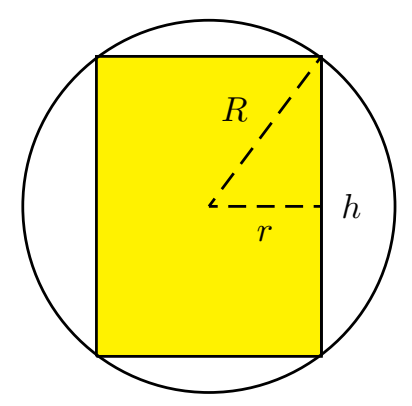


9. Find the largest right circular cone contained in a sphere of radius $R$.

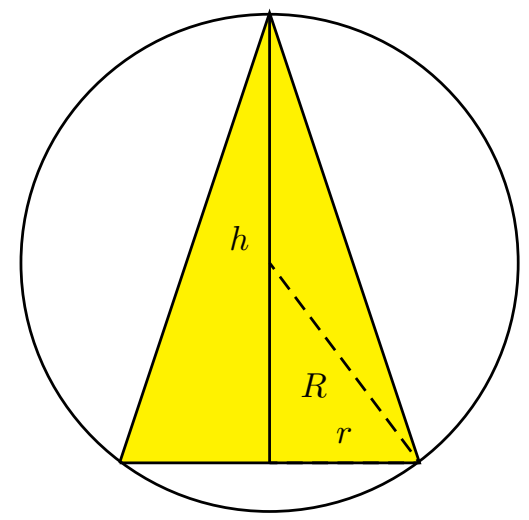


10. Two corridors of widths $a$ and $b$ meet at a right-angled corner. What is the length of the longest ladder which may be carried round the corner? Assume the workman sufficiently unimaginative to keep the ladder horizontal).

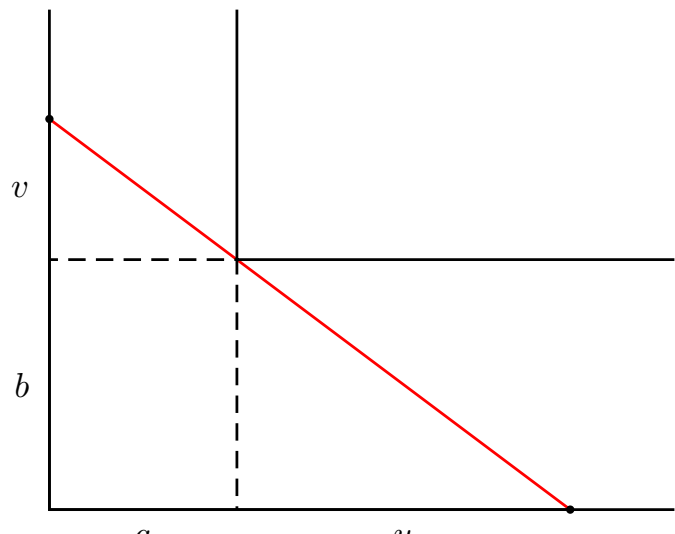

$a$

$u$ 


\section{Chapter 34}

\section{A British test of teachers' mathematical background}

Samples from a test on the mathematical background of (British) teachers in training in the late 1960's. ${ }^{1}$

1. A necessary condition for the truth of a statement $\mathcal{P}$ is that $\beta \geq 7$.

Which one of the following statement must be true?
A If $\beta \geq 7, \mathcal{P}$ is true.
B If $\beta \geq 7, \mathcal{P}$ is false.
$\mathrm{C}$ If $\beta<7, \mathcal{P}$ is true.
D If $\beta<7, \mathcal{P}$ is false.
E None of these.

Answer:

2. A sufficient condition for the truth a statement $\mathcal{Q}$ is that $\beta<0$. Which one of the following statements must be true?
A If $Q$ is true, $\beta \geq 0$.
B If $Q$ is false, $\beta \geq 0$.
C If $\mathcal{Q}$ is true, $\beta<0$.
$\mathrm{D}$ If $Q$ is false, $\beta<0$.
E None of these.

Answer:

\footnotetext{
${ }^{1}$ Math. Gazette, 53 (1969) 357-362.
} 
3. "The condition for a quadrilateral to be cyclic is that the opposite angles must be supplementary".

Which of the words in boldfont should be altered?
A The
B quadrilateral
C cyclic
D supplementary
E None of them

Answer:

4. What is the value of $\frac{x^{2}-9}{x-3}$ when $x=3$ ?
A 0
B 1
C 6
$\mathrm{D} \infty$
E Do not know

Answer:

5. A certain theorem in Euclidean geometry has been proved. Which of the following statements is necessarily true?
A The converse is true and does not require proof.
$\mathrm{B}$ The converse is true but requires proof.
$\mathrm{C}$ The converse is false and does not require dis-proof.
D The converse is false but requires dis-proof.
E None of the above.

Answer: 
6. It has been proved that an infinite number of triangles possess a property 2 .

Statement S: All triangles possess property $Q$.

Which of the following is necessarily correct?

A $\mathcal{S}$ is true, and no further proof is required.

$\mathrm{B} \mathcal{S}$ is true, but proof is required.

$\mathrm{C} S$ is more likely to be true than false.

$\mathrm{D} \mathcal{S}$ is more likely to be false than true.

E None of the above.

Answer:

7. Consider the following calculation (all logarithms to base $e$ ):

$$
\begin{aligned}
\int_{-5}^{-3} \frac{d x}{x+1} & =[\log (x+1)]_{-5}^{-3} \\
{[\log (x+1)]_{-5}^{-3} } & =\log (-2)-\log (-4) \\
\log (-2)-\log (-4) & =\log \left(\frac{-2}{-4}\right) \\
\log \left(\frac{-2}{-4}\right) & =-\log 2
\end{aligned}
$$

In which line, if any, does the first mistake in the calculation occur?
A (I)
B (II)
C (III)
D (IV)
$\mathrm{E}$ None of these: it is correct.

Answer: 
8. An algebraic problem involves $a, b, c$, and we have to write the sum of the products, two at a time.

With which of the following statements do you agree?
A The best order is $a b+a c+b c$.
$\mathrm{B}$ The best order is $a c+b a+c b$.
$\mathrm{C}$ The best order is $b c+c a+a b$.
$\mathrm{D}$ The best order is $c b+a b+a c$.
E There is no "best" order.

Answer:

9. We wish to letter the points $1,2,3$ in the diagram with the letter $L$, $M, N$ in some order.

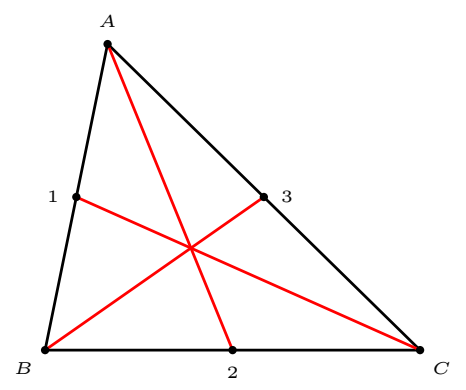

With which of the following statements do you agree;

A The best order is $1 L, 2 M, 3 N$.

B The best order is $1 M, 2 N, 3 L$.

$\mathrm{C}$ The best order is $1 N, 2 L, 3 M$.

D The best order is $1 L, 2 N, 3 M$.

E There is no "best" order.

Answer: 


\section{Chapter 35}

\section{A mathematical contest}

\section{Canadian Invitational Mathematics Challenge ${ }^{1}$}

1 Thirty years ago, the ages of Xaviere, Yolanda, and Zoë were in the ratio 1:2:5. Today, the ratio of Xavior's age to Yolanda's age is 6:7. What is Zoë's present age?

${ }^{1}$ Grades 10 and 11; Crux Math., 29 (2003) 129-132. 
2 Solve the system of equations

$$
\begin{aligned}
x+y+z & =2, \\
x^{2}-y^{2}+z^{2} & =2, \\
x-3 y^{2}+z & =0 .
\end{aligned}
$$


3(a) A flat mirror is perpendicular to the $x y$-plane and stands on the line $y=x+4$. A laser beam from the origin strikes the mirror at $P(-1,3)$ and is reflected to the point $Q$ on the $x$-axis. Determine the coordinates of the point $Q$.

3(b) A flat mirror is perpendicular to the $x y$-plane and stands on a line $\mathcal{L}$. A laser beam from the origin strikes the mirror at $P(-1,5)$ and is reflected to the point $Q(24,0)$. Determine the equation of the line $\mathcal{L}$. 
4 Determine all pairs of nonnegative integers $(m, n)$ which are solutions to the equation $3\left(2^{m}\right)+1=n^{2}$.

5 Let $f(n)=n^{4}+2 n^{3}-n^{2}+2 n+1$.

(a) Show that $f(n)$ can be written as the product of two quadratic polynomials with integer coefficients.

(b) Determine all integers $n$ for which $|f(n)|$ is a prime number. 


\section{Chapter 36}

\section{Some geometry problems from recent journals}

1 Crux Mathematicorum

Jim Totten

Department of Mathematics and Statistics

University College of the Cariboo

Kamloops, BC, Canada, V2C 4Z9

2 Mathematics Magazine

Elgin Johnston, Problem Editor

Department of Mathematics

Iowa State University

Ames, IA 50011

3 American Mathematical Monthly

Doug Hensley, Monthly Problems

Department of Mathematics

Texas A\&M University

3368 TAMU

College Station, TX 77843-3368

4 Pi Mu Epsilon Journal

Michael McConnell

840 Wood Street

Mathematics Department

Clarion University

Clarion, PA 16214 
Crux 2813, proposed by Barry Monson, University of New Brunswick, Fredericton, NB and J. Chris Fisher, University of Regina, Regina, SK, Canada

Suppose that $M$ is the midpoint of side $A B$ of the square $A B C D$. Let $P$ and $Q$ be the points of intersections of the line $M D$ with the circle, center $M$, radius $M A(=M B)$, where $P$ is inside the square and $Q$ is outside. Prove that the rectangle $A P B Q$ is a golden rectangle, that is,

$$
P B: P A=(\sqrt{5}+1): 2 \text {. }
$$


Crux 2822, proposed by Peter Woo, Biola University, La Mirada, CA

Suppose that $\Pi$ is a parallelogram with sides of lengths $2 a$ and $2 b$ and with acute angle $\alpha$, and that $F$ and $F^{\prime}$ are the foci of the ellipse $\Lambda$ that is tangent to the four sides of $\Pi$ at their midpoints.

(a) Find the major and minor semi-axes of $\Pi$ in terms of $a, b$ and $\alpha$.

(b) Find a straight-edge and compass construction for $F$ and $F^{\prime}$. 
Crux 2823, proposed by Christopher J. Bradley, Clifton COllege, Britol, UK

Suppose that $L, M, N$ are points on $B C, C A, A B$ respectively, and are distinct from $A, B, C$. Suppose further that

$$
\frac{B L}{L C}=\frac{1-\lambda}{\lambda}, \quad \frac{C M}{M A}=\frac{1-\mu}{\mu}, \quad \frac{A N}{N B}=\frac{1-\nu}{\nu},
$$

and that the circles $A M N, B N L$, and $C L M$ meet at the Miquel point $P$. Find $[B C P]:[C A P]:[A B P]$ in terms of $\lambda, \mu, \nu$ and the side lengths of triangle $A B C$. 


\section{Crux 2830, proposed by D. J. Smeenk, Zaltbommel, the Netherlands}

Suppose that $\Gamma(O, R)$ is the circumcircle of triangle $A B C$. Suppose that side $A B$ is fixed and that $C$ varies on $\Gamma$ (always on the same side of $A B$ ).

Suppose that $I_{a}, I_{b}, I_{c}$ are the centers of the excircles of triangle $A B C$ opposite $A, B, C$ respectively. If $\Omega$ is the center of the circumcircle of triangle $I_{a} I_{b} I_{c}$, determine the locus of $\Omega$ as $C$ varies. 


\section{Crux 2836, proposed by G. Tsintsifas, Thessaloniki, Greece}

Suppose that triangle $A B C$ is equilateral and that $P$ is an interior point. The lines $A P, B P, C P$ intersect the opposite sides at $D, E, F$ respectively. Suppose that $P D=P E=P F$. Determine the locus of $P$.

Suppose $P$ has homogeneous barycentric coordinates $(x: y$ :

$z)$ with respect to triangle $A B C$. Then, $D$ divides $B C$ in the ratio $B P: P C=z: y$, and $P D=\frac{x}{x+y+z} \cdot A D$. By Stewart's theorem,

$$
A D^{2}=\frac{z}{y+z} a^{2}+\frac{y}{y+z} a^{2}-\frac{y z}{(y+z)^{2}} a^{2}=\frac{y^{2}+y z+z^{2}}{(y+z)^{2}} \cdot a^{2} .
$$

Therefore,

$$
P D^{2}=\frac{x^{2}\left(y^{2}+y z+z^{2}\right)}{(x+y+z)^{2}(y+z)^{2}} \cdot a^{2} .
$$

Similarly, we obtain

$$
\begin{aligned}
& P E^{2}=\frac{y^{2}\left(z^{2}+z x+x^{2}\right)}{(x+y+z)^{2}(z+x)^{2}} \cdot a^{2}, \\
& P F^{2}=\frac{z^{2}\left(x^{2}+x y+y^{2}\right)}{(x+y+z)^{2}(x+y)^{2}} \cdot a^{2} .
\end{aligned}
$$

Therefore, $P E=P F$ if and only if

$$
(x+y)^{2} y^{2}\left(z^{2}+z x+x^{2}\right)=(z+x)^{2} z^{2}\left(x^{2}+x y+y^{2}\right),
$$

or

$$
(y-z)(x+y+z)\left(x^{3}(y+z)+x^{2}\left(y^{2}+y z+z^{2}\right)+x y z(y+z)+y^{2} z^{2}\right)=0 .
$$

Since $P$ is an interior point, $x, y, z$ are positive. We conclude that $y=z$. Similarly, $P F=P D$ if and only if $z=x$.

The point $P$ has coordinates $x: y: z=1: 1: 1$; it is the center of the equilateral triangle. 
Crux 2840, proposed by Juan-Bosco Romero Márquez, University of Valladolid, Valldolid, Spain

Let $A^{\prime}$ be an interior point of the line segment $B C$ in triangle $A B C$. The interior bisectors of $\angle B A^{\prime} A$ and $\angle C A^{\prime} A$ intersect $A B$ and $C A$ at $D$ and $E$ respectively. Prove that $A A^{\prime}, B E$, and $C D$ are concurrent. 


\section{Crux 2847, proposed by G. Tsintsifas, Thessaloniki, Greece}

The inscircle inscribed in a tetrahedron is a circle of maximum radius inscribed in the tetrahedron, considering every possible orientations in $\mathbb{E}^{3}$.

Find the radius of the inscircle of a regular tetrahedron. 


\section{Crux 2849, proposed by Toshio Seimiya, Kawasaki, Japan}

In a convex quadrilateral $A B C D$, we have $\angle A B C=\angle B C D=120^{\circ}$. Suppose that $A B^{2}+B C^{2}+C D^{2}=A D^{2}$. Prove that $A B C D$ has an inscribed circle. 
Mathematics Magazine, Problem 1669, proposed by A. N. Duman, Bilkent University, Turkey

Let $A B C$ be a triangle and let $E$ be the midpoint of $B C$. A circle passing through $A$ and $C$ intersects $B A$ and $B C$ in points $G$ and $E$ respectively. Let $D$ be the midpoint of $E C$. A line through $D$ and perpendicular to $B C$ intersects $A C$ at $F$, with $3 A F=F C$. Prove that triangle $F D G$ is similar to triangle $A B C$. 
Mathematics Magazine, Problem 1671, proposed by M. N. Deshpande, Institute of Science, Nagpur, India

Let $\mathcal{T}$ be the set of triangles $A B C$ for which there is a point $D$ on $B C$ such that segments $A B, B D, A D, D C$, and $A C$ have integral lengths and $\angle A C D=\frac{1}{2} \angle A B C=\frac{1}{3} \angle A D B$.

(a) Characterize the sets $\{a, b, c\}$ that are sets of side lengths of triangles in $\mathcal{T}$.

(b) Find the triangle of minimum area in $\mathcal{T}$. 


\section{American Mathematical Monthly, Problem 11006, proposed by B. Suceavă,} California State University, Fullerton, CA

Let $A B C$ be an acute triangle, $T$ the midpoint of arc $B C$ of the circle circumscribing $A B C$. Let $G$ and $K$ be the projections of $A$ and $T$ respectively on $B C$, let $H$ and $L$ be the projections of $B$ and $C$ on $A T$, and let $E$ be the midpoint of $A B$. Prove that

(a) $K H / / A C, G L / / B T, G H / / T C$, and $L K / / A B$.

(b) $G, H, K$ and $L$ are concyclic.

(c) The center of the circle through $G, H$, and $K$ lies on the Euler circle (nine-point circle) of triangle $A B C$. 
Pi Mu Epsilon Journal, Problem 1058, proposed by P. A. Lindstrom, Batavia, NY

Suppose that triangle $A B C$ has an interior point $P$. Let $D, E, F$ be points on sides $A B, B C, C A$ respectively, so that $P D \perp A B, P E \perp$ $B C, P F \perp C A$. Let $|A B|=x,|B C|=y,|C A|=z,|A B|=a$, $|B E|=b$, and $|C F|=c$.

1. Show that $(x-a)^{2}+(y-b)^{2}+(z-c)^{2}=a^{2}+b^{2}+c^{2}$.

2. Show that if $\triangle A B C$ is an equilateral triangle, then $a+b+c=$ $\frac{1}{2}$ (perimeter) of triangle $A B C$. 
Pi Mu Epsilon Journal, Problem 1060, proposed by A. B. Ayoub, Pennsylvania State University, Abington, College, Abington, PA

Suppose $\triangle A B C$ is an equilateral triangle. The points $D, E$, and $F$ are on $A B, B C, C A$ respectively such that $|A D|=|B E=| C F \mid$. Show that the circumcircles of triangles $A B C$ and $D E F$ are concentric. 


\section{Chapter 37}

\section{The Josephus problem and its generalization}

\subsection{The Josephus problem}

$n$ prisoners are arranged in a circle. In succession, every second one is removed from the circle and executed, and the last one is set free. Who is the survivor?

\section{Examples}

1. $n=10$ :

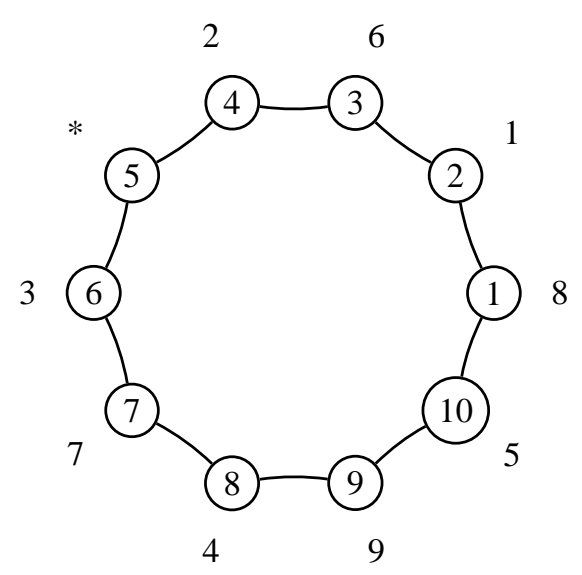

2. $n=21$. After the removal of the 10 even numbered ones and then the first, there are the 10 odd numbers $3,5, \ldots, 19,21$. The survivor is the 5-th of this list, which is 11 . 
Theorem 37.1. Let $f(n)$ be the survivor in the Josephus problem of $n$ prisoners.

$$
\begin{aligned}
f(2 n) & =2 f(n)-1, \\
f(2 n+1) & =2 f(n)+1 .
\end{aligned}
$$

\section{Example}

$$
\begin{aligned}
f(100) & =2 f(50)-1 \\
& =2(2 f(25)-1)-1=4 f(25)-3 \\
& =4(2 f(12)+1)-3=8 f(12)+1 \\
& =8(2 f(6)-1)+1=16 f(6)-7 \\
& =16(2 f(3)-1)-7=32 f(3)-23 \\
& =32(2 f(1)+1)-23=64 f(1)+9 \\
& =73 .
\end{aligned}
$$

There is an almost explicit expression for $f(n)$ : if $2^{m}$ is the largest power of $2 \leq n$, then

$$
f(n)=2\left(n-2^{m}\right)+1 .
$$

Corollary 37.2. The binary expansion of $f(n)$ is obtained by transferring the leftmost digit 1 of $n$ to the rightmost.

$$
f(100)=f\left(1100100_{2}\right)=1001001_{2}=64+8+1=73 .
$$


37.2 Generalized Josephus problem $\mathcal{J}(n, k)$

$\mathcal{J}(10,3)$ :

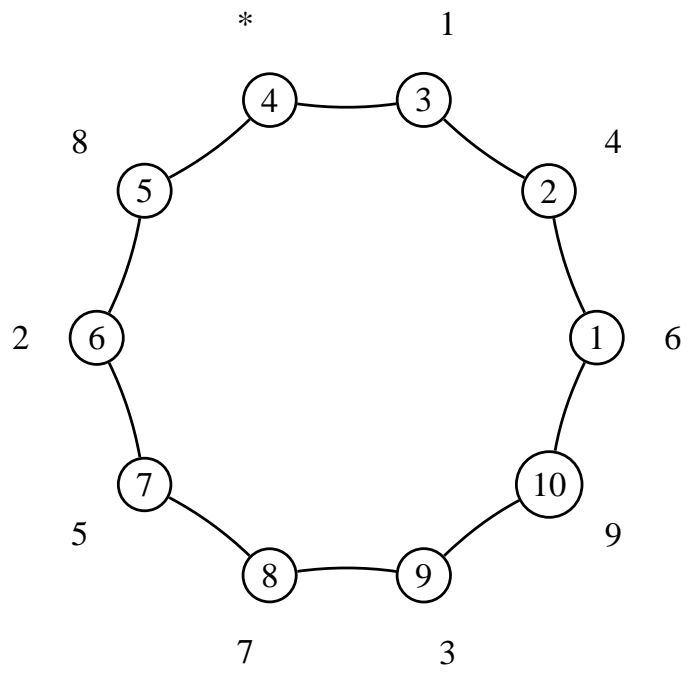

\section{$\mathcal{J}(10, k)$ for various values of $k$}

For $n=10$, here are the sequences of execution depending on the values of $k$. The last column gives the survivors.

\begin{tabular}{|l||ccccccccc|c|}
\hline$k$ & & & & & & & & & $*$ \\
\hline 1 & 1 & 2 & 3 & 4 & 5 & 6 & 7 & 8 & 9 & 10 \\
\hline 2 & 2 & 4 & 6 & 8 & 10 & 3 & 7 & 1 & 9 & 5 \\
\hline 3 & 3 & 6 & 9 & 2 & 7 & 1 & 8 & 5 & 10 & 4 \\
\hline 4 & 4 & 8 & 2 & 7 & 3 & 10 & 9 & 1 & 6 & 5 \\
\hline 5 & 5 & 10 & 6 & 2 & 9 & 8 & 1 & 4 & 7 & 3 \\
\hline 6 & 6 & 2 & 9 & 7 & 5 & 8 & 1 & 10 & 4 & 3 \\
\hline 7 & 7 & 4 & 2 & 1 & 3 & 6 & 10 & 5 & 8 & 9 \\
\hline 8 & 8 & 6 & 5 & 7 & 10 & 3 & 2 & 9 & 4 & 1 \\
\hline 9 & 9 & 8 & 10 & 2 & 5 & 3 & 4 & 1 & 6 & 7 \\
\hline 10 & 10 & 1 & 3 & 6 & 2 & 9 & 5 & 7 & 4 & 8 \\
\hline
\end{tabular}

Positions 2 and 6 are deadly positions for the Josephus problem of 10 prisoners and random choice of $k$. 


\section{Exercise}

1. For what values of $n$ is $f(n)=n$ ?

2. For what values of $n$ is $f(n)=n-1$ ?

3. Make a list of the deadly positions of the Josephus problem for $n=4,5, \ldots, 9$.

4. For $n=7$, there is only one deadly position 1 . This means that one other position is most likely to survive? Which one is it?

5. Find out the survivor in the Josephus problem $\mathcal{J}(24,11)$.

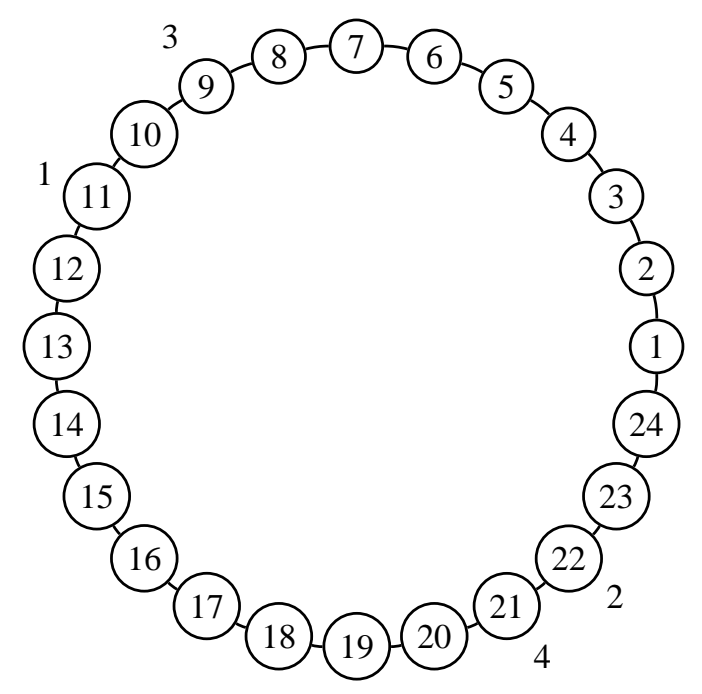

6. The deadly positions for $\mathcal{J}(24, k), k=1, \ldots, 24$ are $5,12,13,16$, $18,19,22$. What is the one with the best chance of survival? 


\section{Chapter 38}

\section{Permutations}

\subsection{The universal sequence of permutations}

For convenient programming we seek an enumeration of the permutations. Regard each permutation of $1,2, \ldots n$ as a bijection $\pi: \mathbb{N} \rightarrow \mathbb{N}$ which is "eventually" constant, i.e., $f(m)=m$ for $m>n$. The enumeration begins with the identity permutation. The permutations of 1 , $2, \ldots n$ are among the first $n$ ! of them, and each of the first $(n-1)$ ! permutations ends with $n$.

Given an integer $m$, we write

$$
m-1=r_{2} \times 1 !+r_{3} \times 2 !+\cdots+r_{k} \times(k-1) !
$$

for $0 \leq r_{i}<i$. These can be calculated recursively as follows. Beginning with $\left(q_{1}, r_{1}\right)=(m-1,0)$, we set, for each $i \geq 2$,

$$
q_{i-1}=i \times q_{i}+r_{i}
$$

In other words,

$$
\left(q_{i}, r_{i}\right)=\left(\left\lfloor\frac{q_{i-1}}{i}\right\rfloor, \bmod \left(q_{i-1}, i\right)\right)
$$

Along with these, we construct a sequence of lists which ends at the desired permutation. Let $L_{1}=(1)$. For $i \geq 2$, form $L_{i}$ by inserting $i$ into $L_{i-1}$ so that there are exactly $r_{i}$ members smaller than $i$ on its right hand side. Then $L_{k}$ is the permutation corresponding to $m$. 


\section{Example}

To find the 12345 th permutation, we write

$12344=0 \times 1 !+1 \times 2 !+1 \times 3 !+4 \times 4 !+0 \times 5 !+3 \times 6 !+2 \times 7 !$.

The corresponding sequences are

\begin{tabular}{|l|l|}
\hline$k$ & $L_{k}$ \\
\hline \hline 1 & $(1)$ \\
2 & $(1,2)$ \\
3 & $(1,3,2)$ \\
4 & $(1,3,4,2)$ \\
5 & $(5,1,3,4,2)$ \\
6 & $(5,1,3,4,2,6)$ \\
7 & $(5,1,3,7,4,2,6)$ \\
8 & $(5,1,3,7,4,8,2,6)$ \\
\hline
\end{tabular}

The permutation is $(5,1,3,7,4,8,2,6)$. 


\subsection{The position of a permutation in the universal se- quence}

Given a permutation $L_{n}$, we want to determine its position in the enumeration scheme above. For $j=n, n-1, \ldots, 2$, let

(i) $r_{j}$ be the number of elements in $L_{j}$ on the right hand side of $j$,

(ii) $L_{j-1}$ be the sequence $L_{j}$ with $j$ deleted.

Then, the position number of the permutation $L_{n}$ is

$$
1+r_{2} \times 1 !+r_{3} \times 2 !+\cdots+r_{n} \times(n-1) ! .
$$

This number can be computed recursively as follows.

$$
\begin{gathered}
s_{n}=r_{n}, \\
s_{n-1}=s_{n} \times(n-1)+r_{k-1}, \\
\vdots \\
s_{j-1}=s_{j} \times(j-1)+r_{j-1}, \\
\vdots \\
s_{2}=s_{2} \times 2+r_{2}, \\
s_{1}=s_{2} \times 1+1 .
\end{gathered}
$$

\section{Example}

Consider the permutation $L=(1,4,6,2,3,7,9,8,5)$.

\begin{tabular}{llll}
$j$ & $L_{j}$ & $r_{j}$ & $s_{j}$ \\
\hline \hline 9 & $(1,4,6,2,3,7,9,8,5)$ & 2 & 2 \\
8 & $(1,4,6,2,3,7,8,5)$ & 1 & 17 \\
7 & $(1,4,6,2,3,7,5)$ & 1 & 120 \\
6 & $(1,4,6,2,3,5)$ & 3 & 723 \\
5 & $(1,4,2,3,5)$ & 0 & 3615 \\
4 & $(1,4,2,3)$ & 2 & 14462 \\
3 & $(1,2,3)$ & 0 & 43386 \\
2 & $(1,2)$ & 0 & 86772 \\
1 & $(1)$ & 1 & 86773
\end{tabular}

This permutation appears as the 86773-th in the universal sequence. 


\section{Exercise}

1. Find the one-billionth permutation in the universal sequence.

2. The inverse permutation of $(5,1,3,7,4,8,2,6)$ is $(2,7,3,5,1,8,4,6)$. What is its position number in the universal sequence?

3. Let $\left(a_{1}, \ldots, 1_{n}\right)$ be a permutation of $(1,2, \ldots, n)$.

(i) $\sum_{\text {cyclic }}\left|a_{i}-a_{i+1}\right| \geq 2 n-2$.

(ii) For how many distinct permutations of $(1,2, \ldots, n)$ does equality hold? Answer: $n \cdot 2^{n}$.

\section{Project: Nice odd integers}

An odd integer $n$ is said to be nice if and only if there is a permutation $\left(a_{1}, a_{2}, \ldots, a_{n}\right)$ of $(1,2, \ldots, n)$ such that the sums

$$
\begin{aligned}
& a_{1}-a_{2}+\cdots-a_{n-1}+a_{n}, \\
& a_{2}-a_{3}+\cdots-a_{n}+a_{1}, \\
& \vdots \\
& a_{n}-a_{1}+\cdots-a_{n-2}+a_{n-1}
\end{aligned}
$$

are all positive. Find all nice integers. 


\section{Chapter 39}

\section{Cycle decompositions}

\subsection{The disjoint cycle decomposition of a permutation}

Theorem 39.1. Every permutation can be decomposed into a product of disjoint cycles.

For example, the permutation

$$
\left(\begin{array}{lllllllllllll}
1 & 2 & 3 & 4 & 5 & 6 & 7 & 8 & 9 & X & J & Q & K \\
6 & 9 & 8 & 3 & 4 & 2 & K & 7 & Q & 5 & 1 & J & X
\end{array}\right)
$$

decomposes into the two disjoint cycles $(1629 Q J)(387 K X 54)$.

Theorem 39.2. Every cycle decomposes into a product of transpositions.

Theorem 39.3. A permutation can be decomposed into a product of transpositions. The parity (of the number of transpositions) of the permutation is independent of the decomposition.

Thus, permutations are classified into even and odd permutations. Even (respectively odd) permutations are said to have parity +1 (respectively -1$)$.

Corollary 39.4. A cycle of length $k$ has parity $(-1)^{k-1}$. More generally, a permutation of $n$ objects has parity

$$
(-1)^{n-\text { number of disjoint cycles in a decomposition. }} \text {. }
$$

In using this formula, the fixed points are counted as 1-cycles, though we usually do not write them in the cycle decomposition of a permutation. 


\subsection{Dudeney's puzzle ${ }^{1}$}

Take nine counters numbered 1 to 9 , and place them in a row in the natural order. It is required in as few exchanges of pairs as possible to convert this into a square number. As an example in six moves we give the following: (7846932), which give the number 139854276, which is the square of 11826 . But it can be done in much fewer moves.

The square of 12543 can be found in four moves, as follows.

\begin{tabular}{|l||lllllllll|}
\hline & 1 & 2 & 3 & 4 & 5 & 6 & 7 & 8 & 9 \\
\hline$(25)$ & 1 & 5 & 3 & 4 & 2 & 6 & 7 & 8 & 9 \\
\hline$(37)$ & 1 & 5 & 7 & 4 & 2 & 6 & 3 & 8 & 9 \\
\hline$(38)$ & 1 & 5 & 7 & 4 & 2 & 6 & 8 & 3 & 9 \\
\hline$(34)$ & 1 & 5 & 7 & 3 & 2 & 6 & 8 & 4 & 9 \\
\hline
\end{tabular}

The squares of 25572, 26733, and 27273 can also be obtained in four moves.

$\bullet$

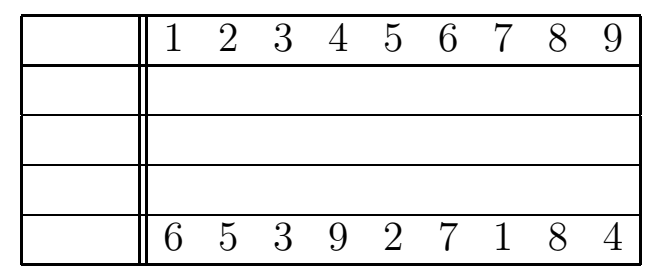

$\bullet$

\begin{tabular}{|c|c|}
\hline & $\begin{array}{lllllllll}1 & 2 & 3 & 4 & 5 & 6 & 7 & 8 & 9\end{array}$ \\
\hline & \\
\hline & \\
\hline & \\
\hline & $\begin{array}{lllllllll}7 & 1 & 4 & 6 & 5 & 3 & 2 & 8 & 9\end{array}$ \\
\hline
\end{tabular}

$\bullet$

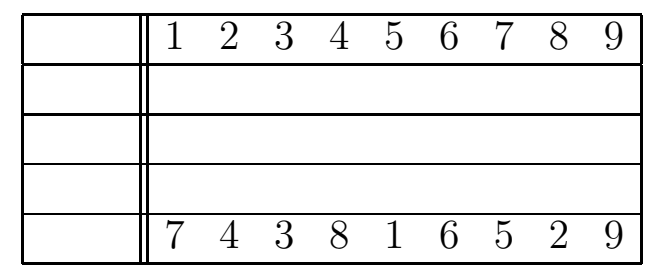

${ }^{1}$ Puzzle 128 of [Dudeney] 
However, there is one which can be made in three moves. Can you find it?

\begin{tabular}{|l||lllllllll|}
\hline & 1 & 2 & 3 & 4 & 5 & 6 & 7 & 8 & 9 \\
\hline & & & & & & & & & \\
\hline & & & & & & & & & \\
\hline & & & & & & & & & \\
\hline
\end{tabular}




\section{The pandigital case of Dudeney's puzzle}

In the pandigital case, there are 4 ways to move

\begin{tabular}{|l||llllllllll|}
\hline & 1 & 2 & 3 & 4 & 5 & 6 & 7 & 8 & 9 & 0 \\
\hline$(37)$ & 1 & 2 & 7 & 4 & 5 & 6 & 3 & 8 & 9 & 0 \\
\hline$(48)$ & 1 & 2 & 7 & 8 & 5 & 6 & 3 & 4 & 9 & 0 \\
\hline$(4 X)$ & 1 & 2 & 7 & 8 & 5 & 6 & 3 & 0 & 9 & 4 \\
\hline$(49)$ & 1 & 2 & 7 & 8 & 5 & 6 & 3 & 0 & 4 & 9 \\
\hline
\end{tabular}

This gives the 1278563049 , the square of 35757 .

There are three other ways of making 4 moves to make a pandigital square. These are the squares of 35853, 38772, and 39147.

\begin{tabular}{|c|c|}
\hline & $\begin{array}{llllllllll}1 & 2 & 3 & 4 & 5 & 6 & 7 & 8 & 9 & 0\end{array}$ \\
\hline & \\
\hline & \\
\hline & \\
\hline & $\begin{array}{llllllllll}1 & 2 & 8 & 5 & 4 & 3 & 7 & 6 & 0 & 9\end{array}$ \\
\hline
\end{tabular}

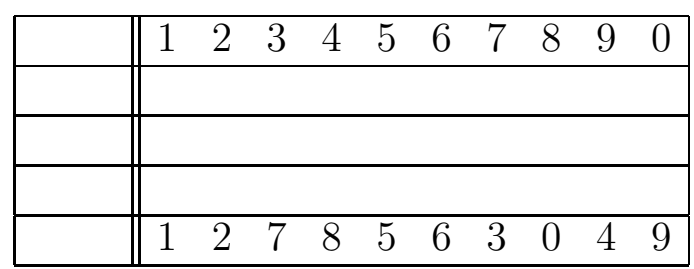

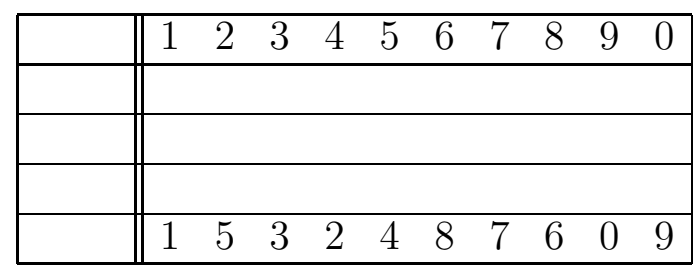




\subsection{Dudeney's Canterbury puzzle 3}

$$
7 \times 28=196=365 .
$$

While $7 \times 28=196$, it is not true that $34 \times 5=196$. Move as few counters as possible to make the equations valid. ${ }^{2}$

\footnotetext{
$2^{`} \mp \times 6 \varepsilon=99 \mathrm{~L}=8 \mathrm{~L} \times \zeta$ 이 $(967)(L \zeta)$
} 


\section{Project}

The multiplication

$$
12 \times 355=6756
$$

is clearly not valid. Move as few counters as possible to make the equation valid. ${ }^{3}$

How about

$$
12 \times 3 \quad 4 \quad 5=65,8,96 ?
$$

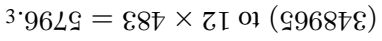




\subsection{The game of ropes and rungs}

Each of the seven players starts by sliding down his own vertical rope, and makes a turn every time he encounters a rung. Who wins the prize *?

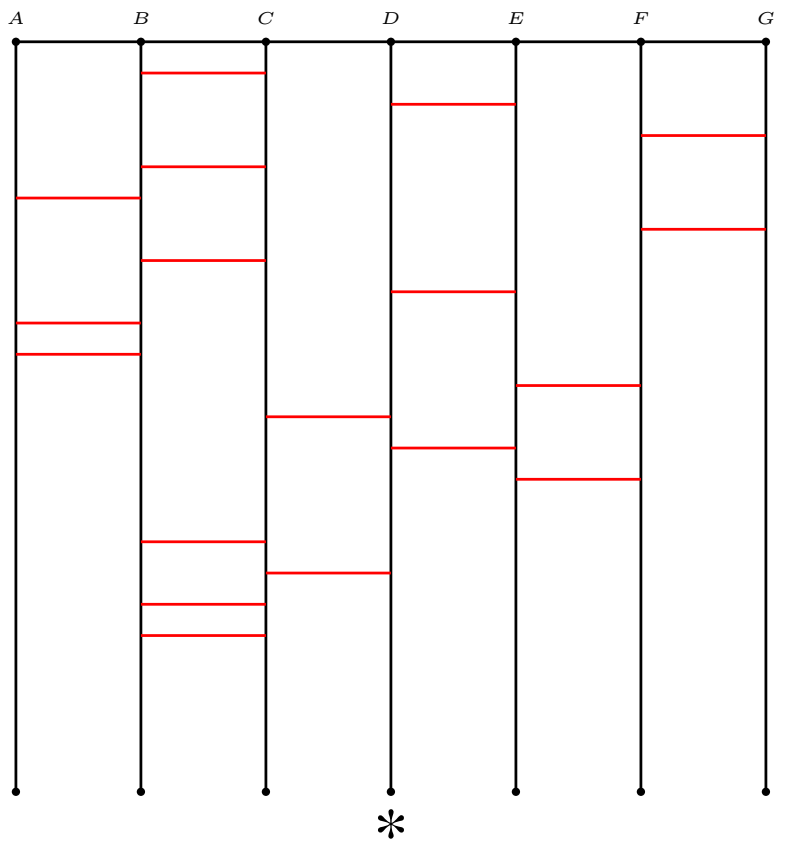

Suppose you are at position $A$, and are permitted to add any number of rungs in any positions, (provided that no two rungs are at the same horizontal level). How would you add rungs to claim the prize ${ }^{*}$ ? 


\section{Chapter 40}

\section{Graph labelling}

\subsection{Edge-magic triangle}

Label the vertices and edges of a triangle with the numbers $1,2, \ldots, 6$ without repitition so that the sum of the three number along each edge is constant.

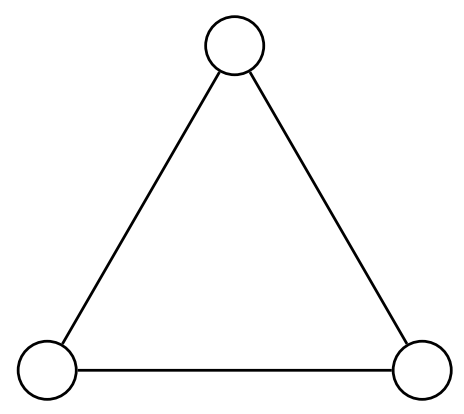




\subsection{Face-magic tetrahedron}

The vertices and edges of a tetrahedron with consecutive integers 1,2 , $\ldots, 10$ so that the four faces all have the same sum 32 .

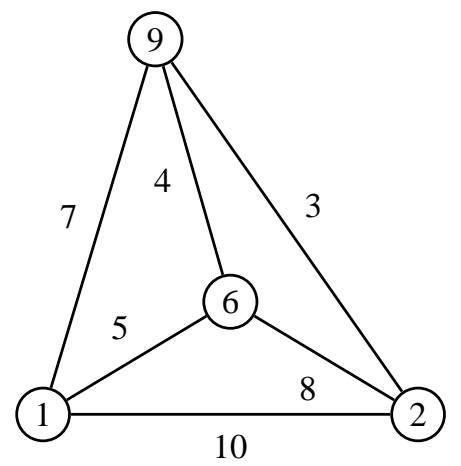

Can you label them with a smaller common face sum?

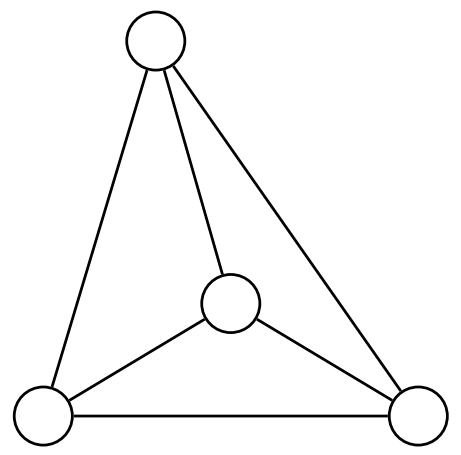




\subsection{Magic cube}

Label the vertices of a cube by the numbers $1,2, \ldots, 8$ without repetition such that the sum of the numbers at the four vertices of each face is a constant.
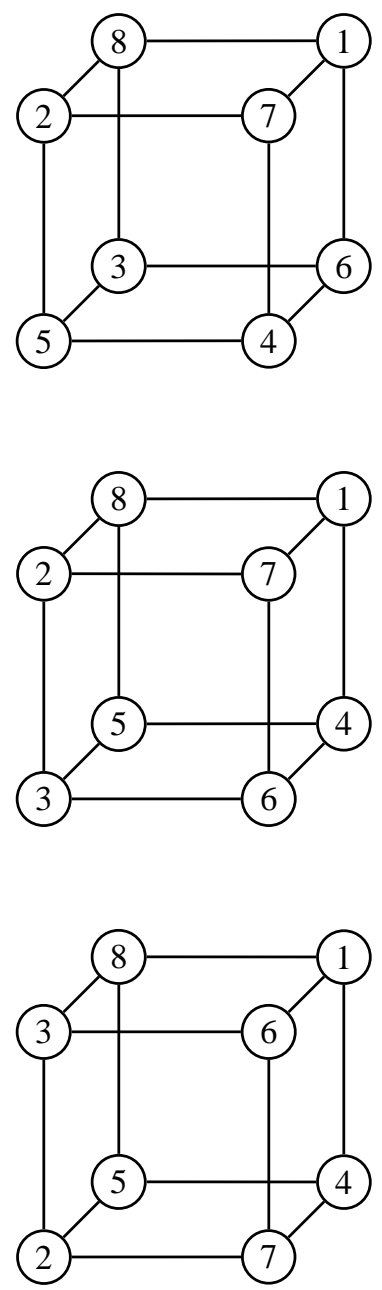


\subsection{Edge-magic heptagon}

Label the vertices and edges with the fourteen numbers $1,2, \ldots, 14$ (without repetition) so that the sum of the three numbers along each edge is constant.

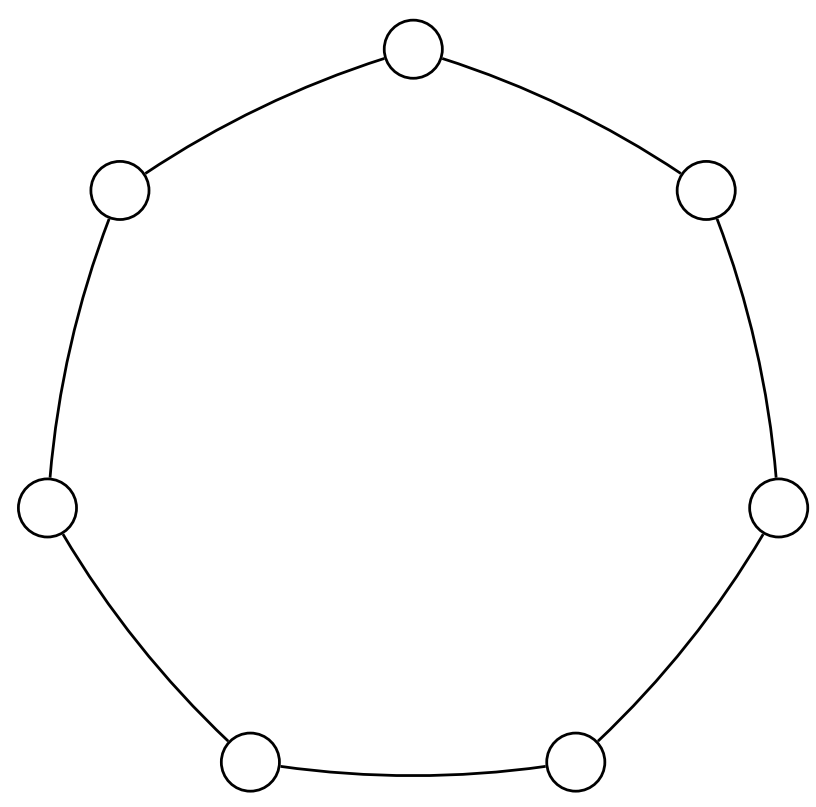




\subsection{Edge-magic pentagram}

It is known that the pentagram cannot be labelled with the numbers 1, 2, $\ldots, 10$ without repetition such that the sum of the four numbers along each line is constant.

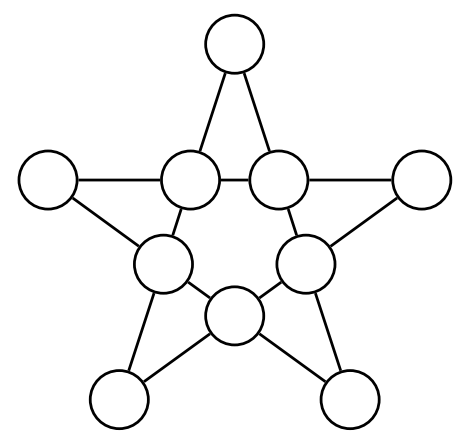

However, given the labelling of the five inner vertices below, it is possible to label the five outer vertices such that the sum of the four vertices along each of the five edges is constant. Find such a labelling with minimum edge sum.

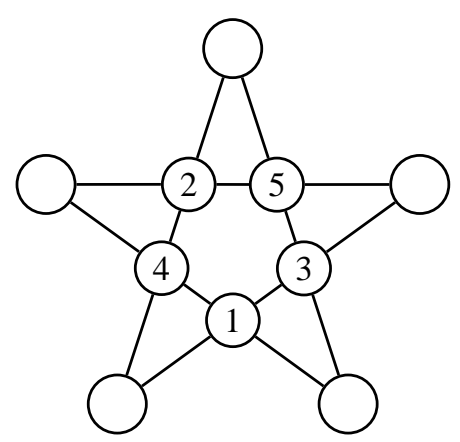




\subsection{A perfect magic circle}

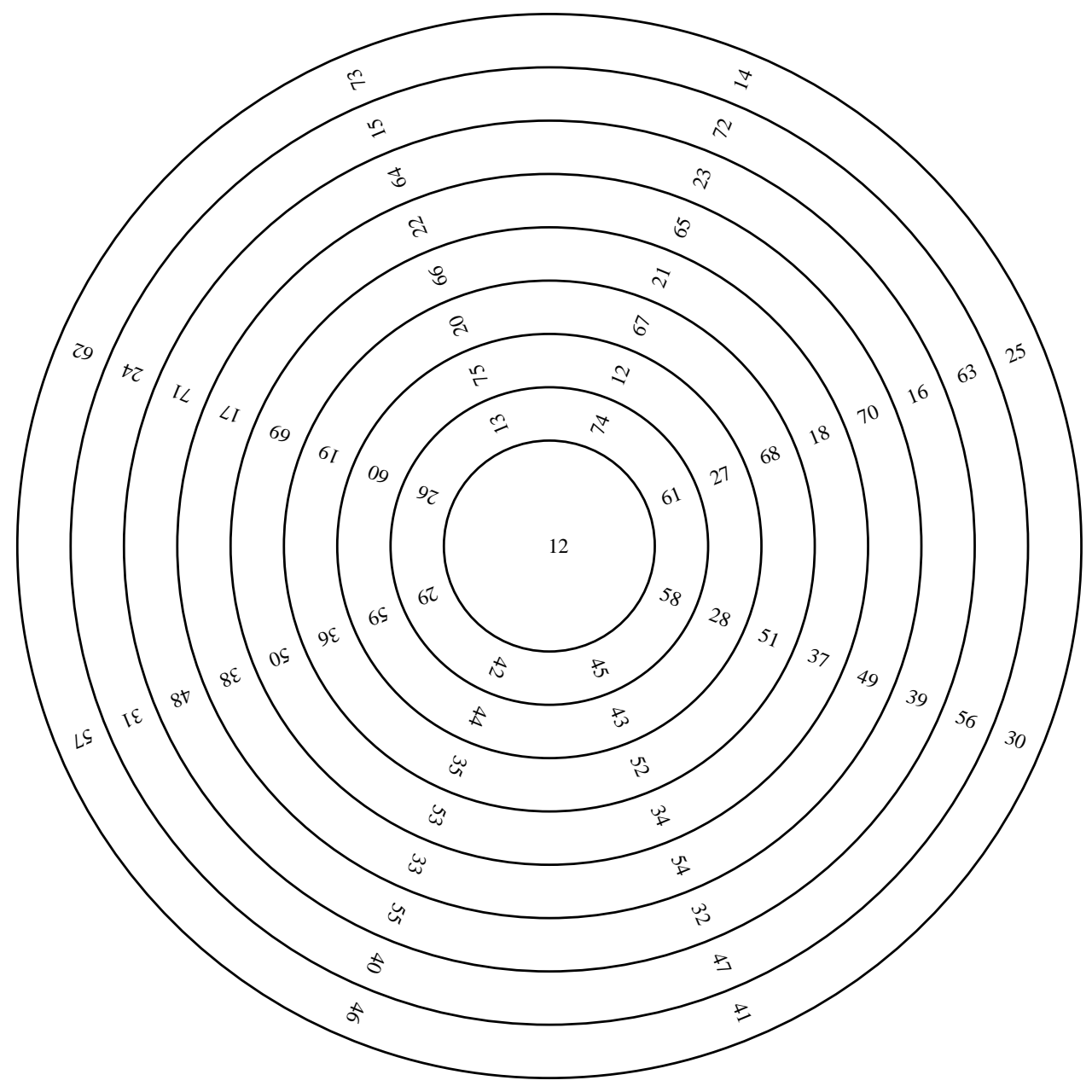




\section{Chapter 41}

\section{Card tricks from permutations}

Consider $m n$ cards arranged in order, from left to right, and from top to bottom. We call this the standard order.

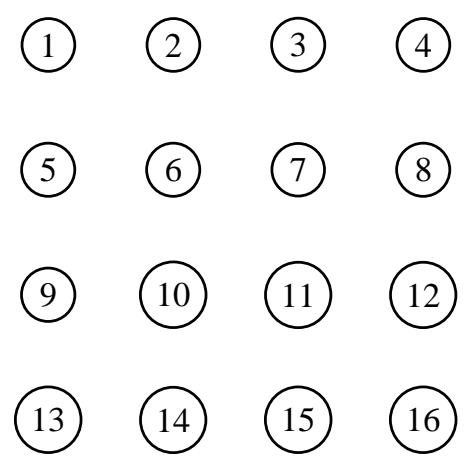

Rearrangements with simple cycle structure lead to interesting card puzzles. For example, the magician asks an audience to note the card at a specific spot, rearranges the cards according to some simple rule, then asks the audience to tell the new card at the spot. The magician is able to tell the card that originally occupies this position. 


\section{The rearrangement $\omega=\omega_{m, n}$}

Pick up the cards along the columns, from top to bottom, and from left to right. Then rearrange them in the standard order.

For $m=n$, this rearrangement is the reflection in the main diagonal.

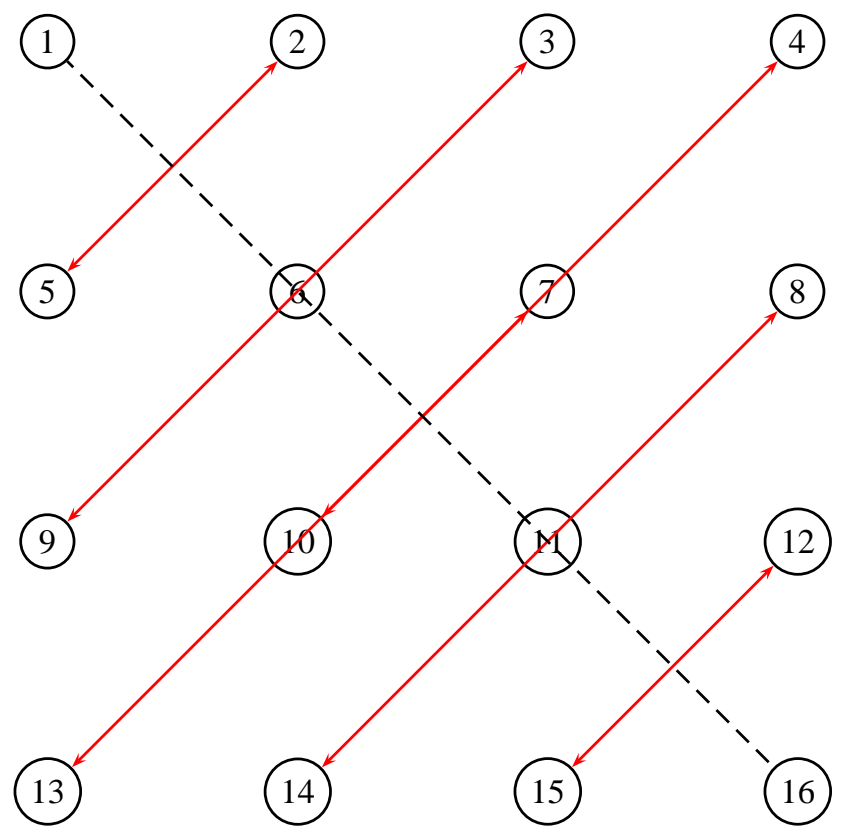


The rearrangement $\rho=\rho_{m, n}$

Pick up the cards along the columns, from bottom to top, and from left to right. Then rearrange them in the standard order.

For $m=n=4$, this rearrangement is the cyclic permutation of the vertices of 4 squares:

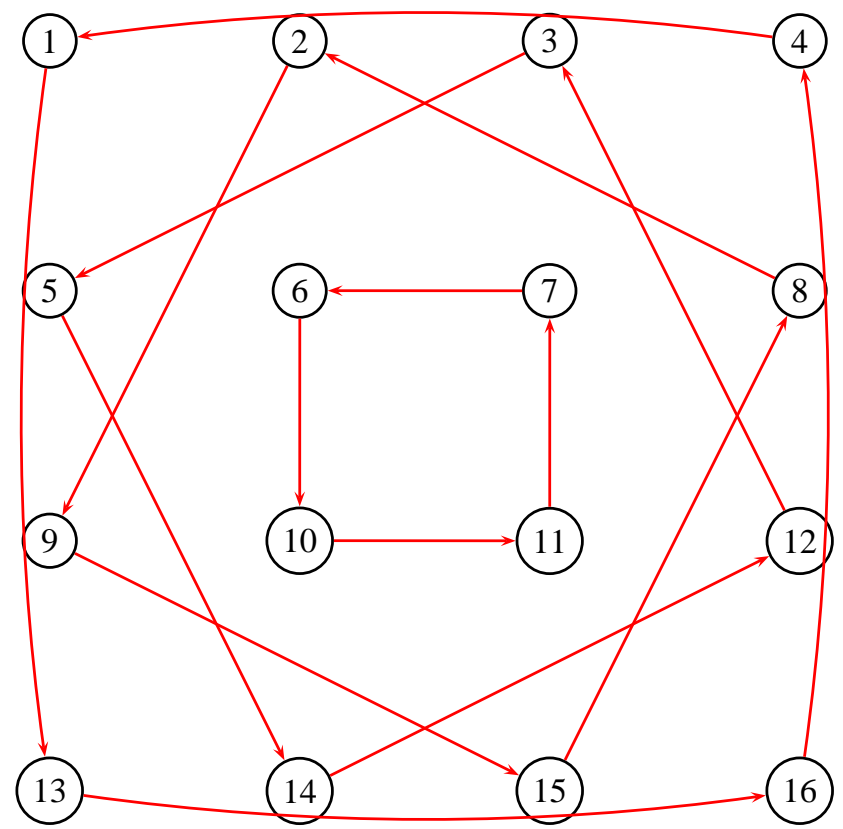

The cycle structure is simpler after one more application: it is simply rotation through $180^{\circ}$ about the center of the square. 


\section{The repeated diagonal rearrangement}

Pick up the cards along the diagonals, from from bottom to top, and from left to right. Then rearrange them in the standard order.

For $m=n=4$, this is the permutation $\delta=\delta_{m, n}$

$$
\begin{array}{cccccccccccccccc}
1 & 2 & 3 & 4 & 5 & 6 & 7 & 8 & 9 & 10 & 11 & 12 & 13 & 14 & 15 & 16 \\
1 & 5 & 2 & 9 & 6 & 3 & 13 & 10 & 7 & 4 & 14 & 11 & 8 & 15 & 12 & 16
\end{array}
$$

with cycle structure

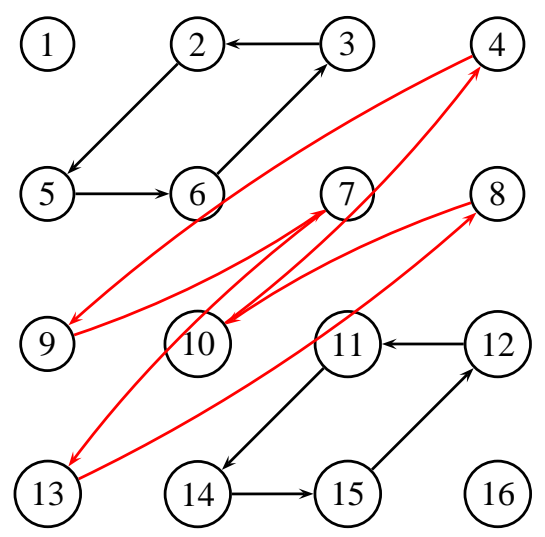

The cycle structure is simpler after one more application:
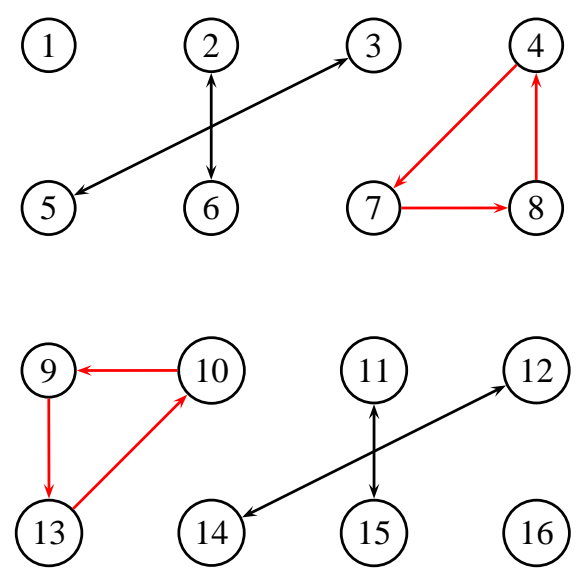


\section{The repeated snake rearrangement}

Pick up the card along the columns, first from top to bottom, then from bottom to top, and alternately. Rearrange in the standard order. This is the permutation $\sigma=\sigma_{m, n}$

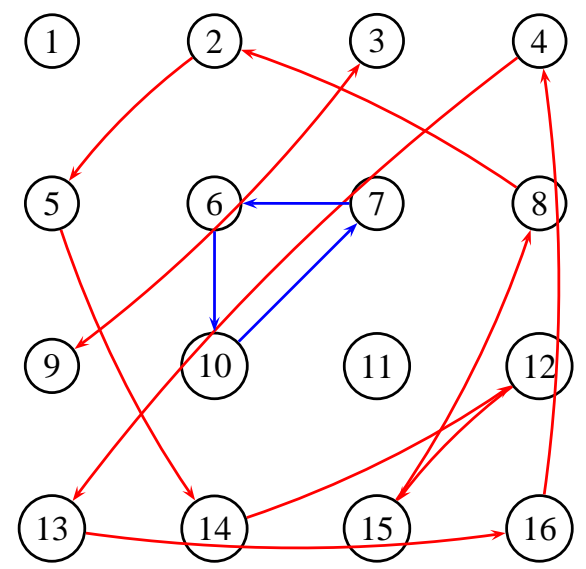

The cycle structure after one more application:

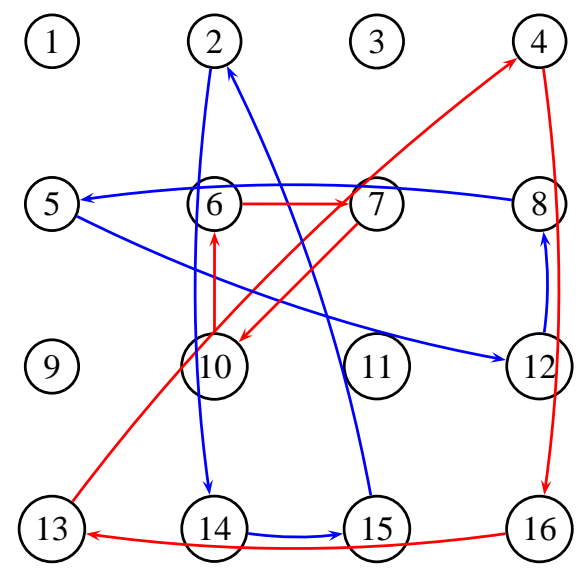




\section{Chapter 42}

\section{Tetrahedra}

\subsection{The isosceles tetrahedron}

An isosceles tetrehedron is one whose four faces are congruent triangles. Given a triangle $A B C$, construct its anticomplimentary triangle $A^{\prime} B^{\prime} C^{\prime}$ by drawing lines through the vertices parallel to their opposite sides. Fold along the sides of the given triangle to bring the vertices $A^{\prime}$, $B^{\prime}, C^{\prime}$ into a point $D$, forming an isosceles tetrahedron $A B C D$. Every isosceles tetrahedron arises from any one of its faces in this way. We may therefore ask for the volume of the isosceles tetrahedron $A B C D$ in terms of the side lengths of triangle $A B C$.
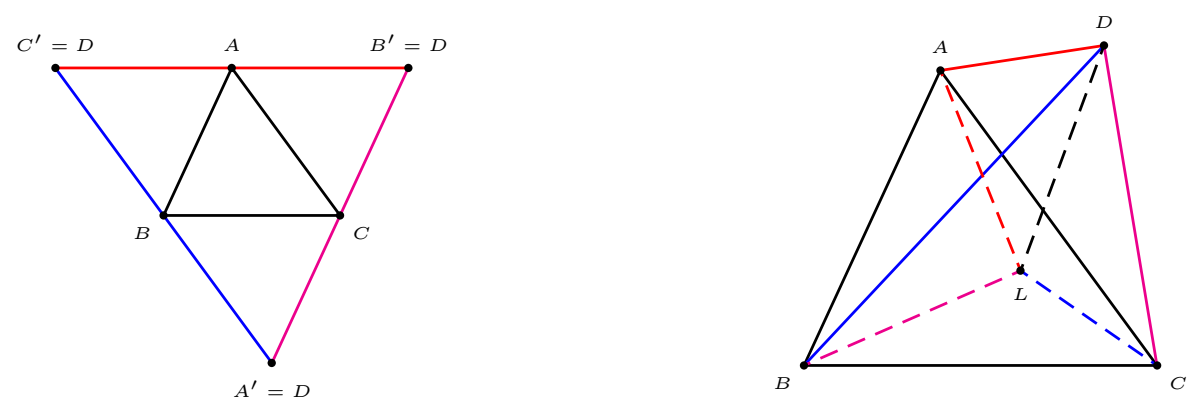

To compute the volume of a tetrahedron, we would drop the perpendicular from a vertex to its opposite face (of area $\Delta$ ) to determine the height $h$ on this face. The volume of the tetrahedron is then $V=\frac{1}{3} \Delta h$. For an isosceles tetrahedron, the position of this perpendicular foot is clearly the same for the four faces. 


\subsection{The volume of an isosceles tetrahedron}

Let $L$ be the pedal of the vertex $D$ on the face $A B C \cdot{ }^{1}$ Consider the plane through $D$ perpendicular to the faces $A B C$ and $D B C$. This is the plane containing $D, L$, and the common pedal $X$ of these points on the line $B C$. Upon unfolding the face $D A B$ into triangle $A B C^{\prime}$, triangle $D Z L$ becomes the segment $C^{\prime} L$ intersecting $A B$ at $Z$. Since $C^{\prime} Z$ is perpendicular to $A B, C^{\prime} L$ is perpendicular to $A^{\prime} B^{\prime}$. The same reasoning applied to the other two faces $D B C$ and $D C A$ shows that $A^{\prime} L, B^{\prime} L$, $C^{\prime} L$ are perpendicular to $B^{\prime} C^{\prime}, C^{\prime} A^{\prime}, A^{\prime} B^{\prime}$ respectively. It follows that $L$ is the orthocenter of triangle $A^{\prime} B^{\prime} C^{\prime}$.
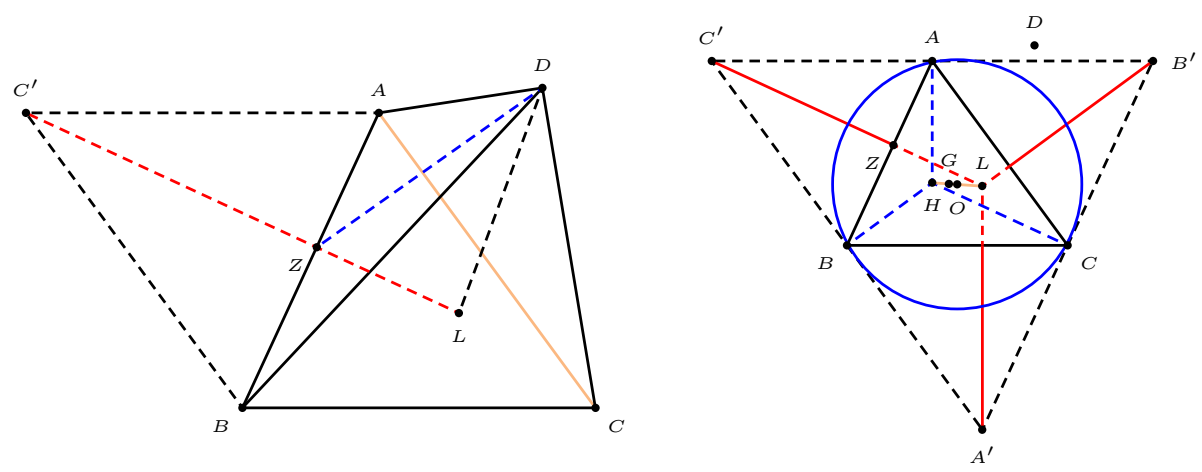

Proposition 42.1. The point $L$ is the reflection of $H$ in $O$.

The point $L$ is called the de Longchamps point of triangle $A B C$.

\section{Proposition 42.2.}

$$
O L^{2}=O H^{2}=R^{2}(1-8 \cos \alpha \cos \beta \cos \gamma) .
$$

Theorem 42.3. The volume of the isosceles tetrahedron on triangle $A B C$ is given by

$$
V_{\mathrm{iso}}=\sqrt{\frac{1}{72}\left(b^{2}+c^{2}-a^{2}\right)\left(c^{2}+a^{2}-b^{2}\right)\left(a^{2}+b^{2}-c^{2}\right)} .
$$

\footnotetext{
${ }^{1}$ We use the word pedal for perpendicular foot or orthogonal projection.
} 


\subsection{Volume of a tetrahedron}

Let $A B C D$ be a tetrahedron with

$$
\begin{aligned}
& B C=a, \quad C A=b, \quad A B=C, \\
& A D=a^{\prime}, \quad B D=b^{\prime}, \quad C D=c^{\prime} \text {. }
\end{aligned}
$$

The volume $V$ of the tetrahedron is given by

$$
\begin{aligned}
V^{2} & =\frac{1}{288}\left|\begin{array}{ccccc}
0 & 1 & 1 & 1 & 1 \\
1 & 0 & a^{\prime 2} & b^{\prime 2} & c^{\prime 2} \\
1 & a^{\prime 2} & 0 & c^{2} & b^{2} \\
1 & b^{\prime 2} & c^{2} & 0 & a^{2} \\
1 & c^{\prime 2} & b^{2} & a^{2} & 0
\end{array}\right| \\
& =\frac{1}{144}\left(\left(\sum_{\text {edges }} a^{2}\right)\left(\sum a^{2} a^{\prime 2}\right)-2 \sum a^{2} a^{\prime 2}\left(a^{2}+a^{\prime 2}\right)-\sum_{\text {faces }} a^{2} b^{2} c^{2}\right) .
\end{aligned}
$$




\subsection{Charles Twigg's envelope model of the tetrahedron 2}

Take an sealed envelope which is two copies of a rectangle $A B C D$ glued along the perimeter. Assume $A B<B C$.

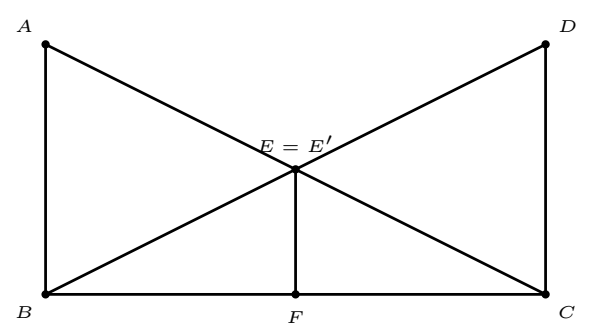

1. Fold the diagonals $A C$ and $B D$. Their intersection is the double point $E$ and $E^{\prime}$.

2. Cut along two half diagonals $A E$ and $D E$ to remove the sector containing one long side and the flap of the envelope.

3. Fold along the remaining portions of the half diagonals ( $B E$ and $C E$ ) and crease firmly. Fold back along the same lines and crease firmly again.

4. Fold $A B$ into $D C$ to form the crease $E F$. Here, $F$ is the midpoint of the side $B C$. Underneath $E$ is the point $E^{\prime}$

5. Separate $E$ and $E^{\prime}$ until $E F E^{\prime}$ is a straight line. Fold up around $E F E^{\prime}$ until $D$ meets $A$, thus forming a hexahedron.

6. Tuck $D$ under $A B$ (or $A$ under $D C$ ) and press up on $B$ and $C$ until $D C$ and $B A$ coincide.

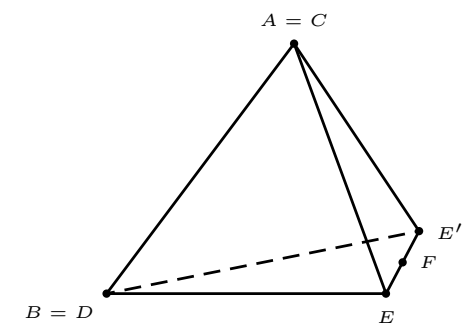

\footnotetext{
${ }^{2}$ C. W. Trigg, Tetrahedral models from envelopes, Math. Mag., 51 (1978) 66-67.
} 


\section{Exercise}

1. Find the volumes of the isosceles tetrahedra with face given below:

\begin{tabular}{|ccc|c|}
\hline$a$ & $b$ & $c$ & $V$ \\
\hline \hline 11 & 20 & 21 & \\
\hline 33 & 65 & 72 & \\
\hline 69 & 91 & 100 & \\
\hline 21 & 99 & 100 & \\
\hline
\end{tabular}

2. Find the volumes of the following tetrahedra:

\begin{tabular}{|ccc|ccc||c|}
\hline$a$ & $b$ & $c$ & $a^{\prime}$ & $b^{\prime}$ & $c^{\prime}$ & $V$ \\
\hline \hline 32 & 33 & 35 & 76 & 70 & 44 & \\
\hline 21 & 32 & 47 & 58 & 76 & 56 & \\
\hline
\end{tabular}

3. What is the shape of the envelope in Twigg's model for which the resulting tetrahedron is regular?

4. In Twigg's envelope model, suppose $A B=2 a$ and $B C=2 b$. What is the volume of the resulting tetrahedron? ${ }^{3}$

\footnotetext{
${ }^{3}$ This is an isosceles tetrahedron with face $2 a, \sqrt{a^{2}+b^{2}}$, and $\sqrt{a^{2}+b^{2}}$. Its volume is $\frac{4}{9} a^{2} \sqrt{b^{2}-a^{2}}$.
} 


\section{Chapter 43}

\section{Lewis Carroll's unused geometry pillow problem}

According to [Rowe], one of the pillow problems Lewis Carroll had attempted but did not include in his collection of pillow problems was the following.

Given a triangle $A B C$, to find, by paper folding, a line $\ell$ which intersects $A C$ and $A B$ at $Y$ and $Z$ respectively) such that if $A^{\prime}$ is the reflection of $A$ in $\ell$, then the reflections of $B$ in $A^{\prime} Z$ and of $C$ in $A^{\prime} Y$ coincide.

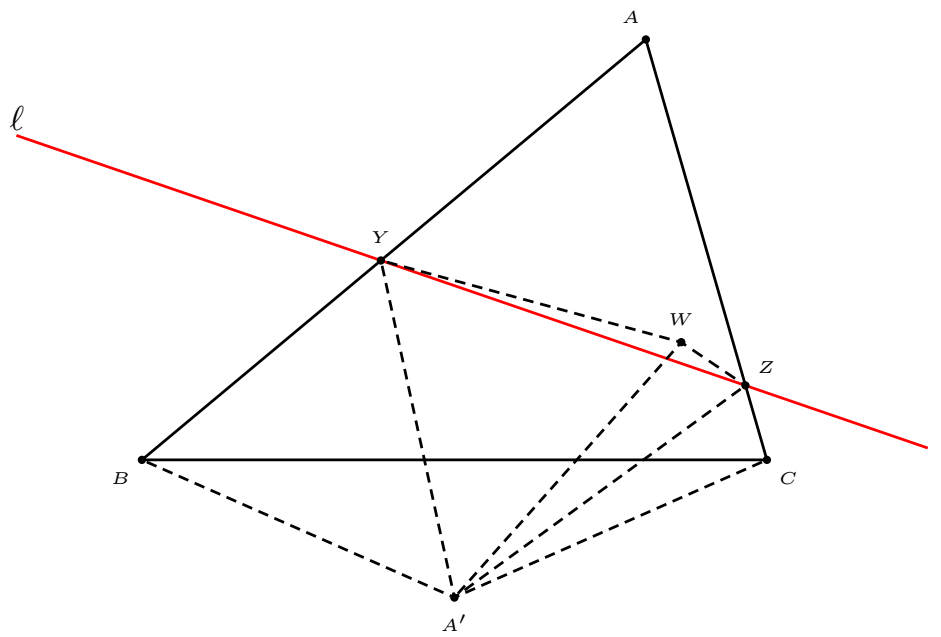

The point $W$ is both the reflection of $B$ in $A^{\prime} Y$, and that of $C$ in $A^{\prime} Z$. It follows that $A^{\prime} B=A^{\prime} W=A^{\prime} C$, and $A^{\prime}$ is on the perpendicular bisector of $B C$. 
Consider the directed angle $\angle B A^{\prime} C$. This is

$$
\begin{aligned}
\angle B A^{\prime} C & =\angle B A^{\prime} W+\angle W A^{\prime} C \\
& =2 \angle Y A^{\prime} W+2 \angle W A^{\prime} Z \\
& =2 \angle Y A^{\prime} Z \\
& =-2 \angle Y A Z
\end{aligned}
$$

since $A^{\prime} Y A Z$ is a rhombus. This means that $\angle B A^{\prime} C=-2 \angle B A C$. The reflection of $A^{\prime}$ in the side $B C$ is therefore the point $Q$ on the perpendicular bisector such that $\angle B Q C=2 \angle B A C$, which is necessarily the circumcenter $O$ of triangle $A B C$. We therefore conclude that $A^{\prime}$ is the reflection of the circumcenter $O$ in the side $B C$, and the reflection line $\ell$ is the perpendicular bisector of the line $A A^{\prime}$.

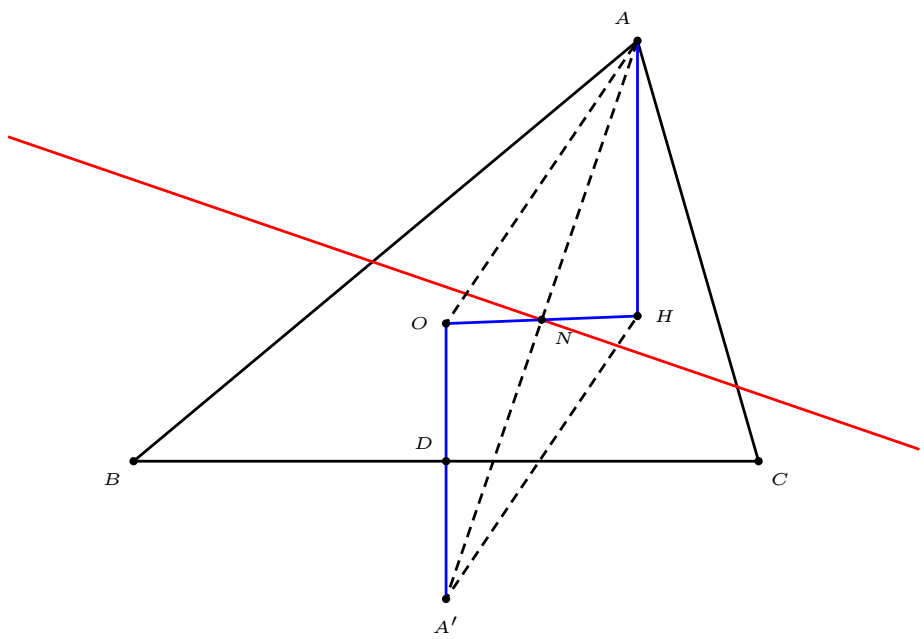

Let $D$ be the midpoint $B C$ and $H$ the orthocenter of triangle $A B C$. In a standard proof of the Euler line theorem, it is established that $A H=$ $2 O D,{ }^{1}$ and that the midpoint of $O H$ is the nine-point center of triangle $A B C$. This means that $A H=O A^{\prime}$, and $A O A^{\prime} H$ is a parallelogram. It follows that the midpoint of $A A^{\prime}$ is the same as that of $O H$, the ninepoint center $N$ of triangle $A B C$. The Lewis Carroll paper-folding line is the perpendicular to $A N$ at $N$.

\footnotetext{
${ }^{1} A H=2 \cdot O D=2 R \cos A$, where $R$ is the circumradius of triangle $A B C$.
} 


\section{Chapter 44}

\section{Japanese Temple Geometry}
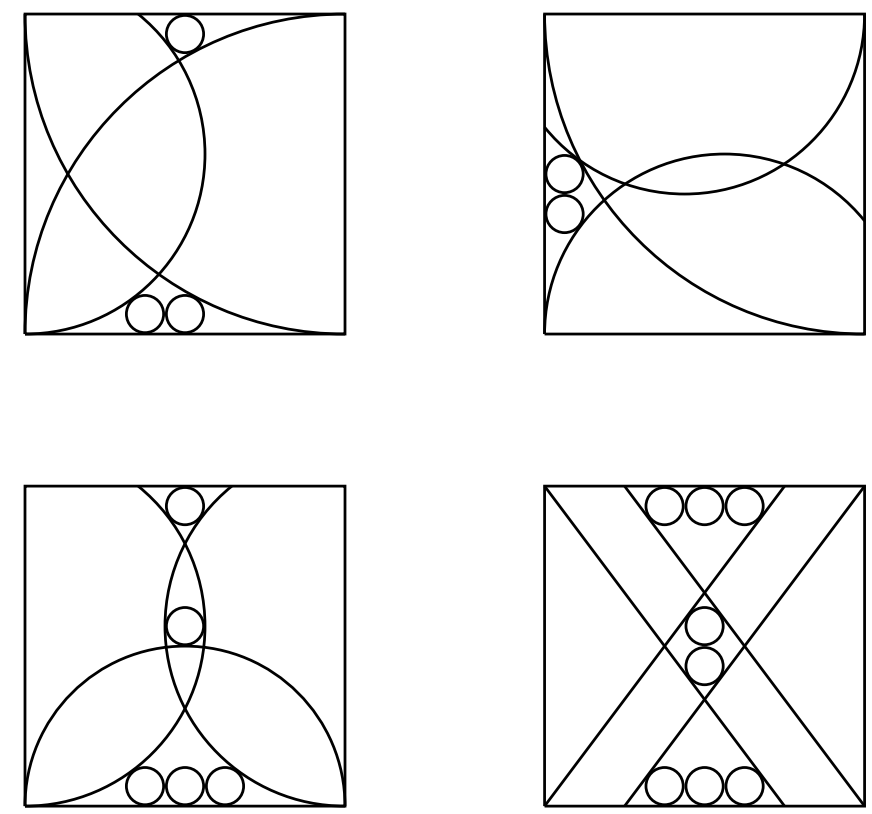

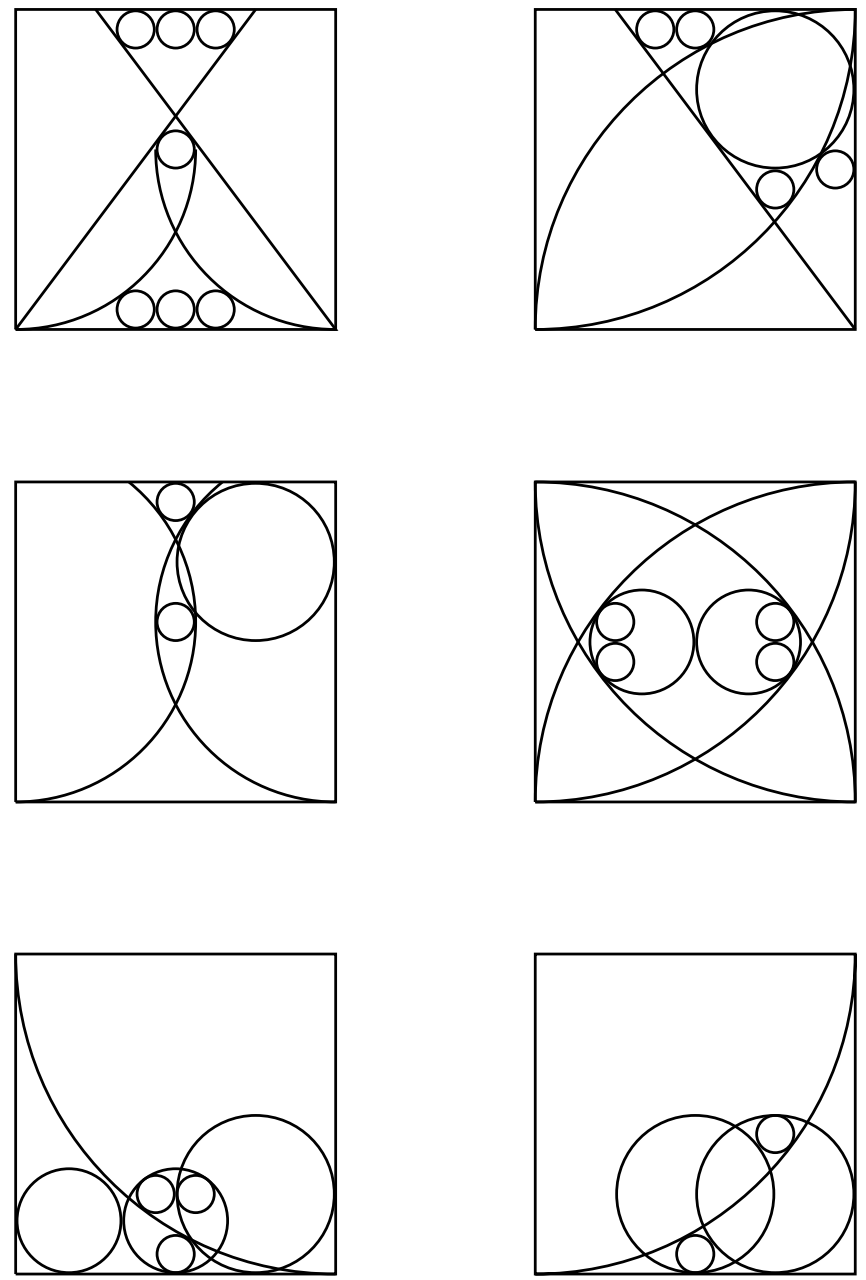

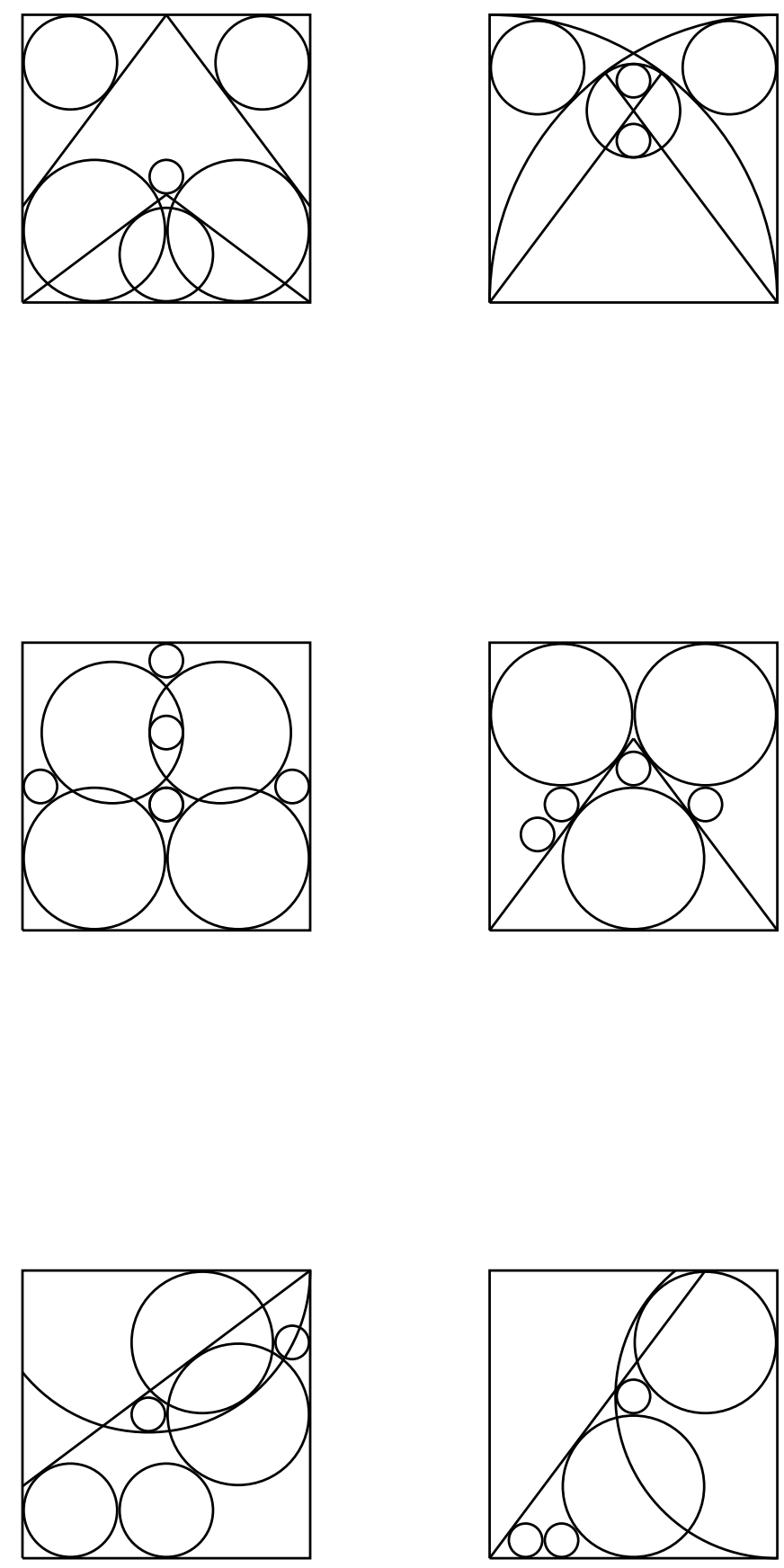

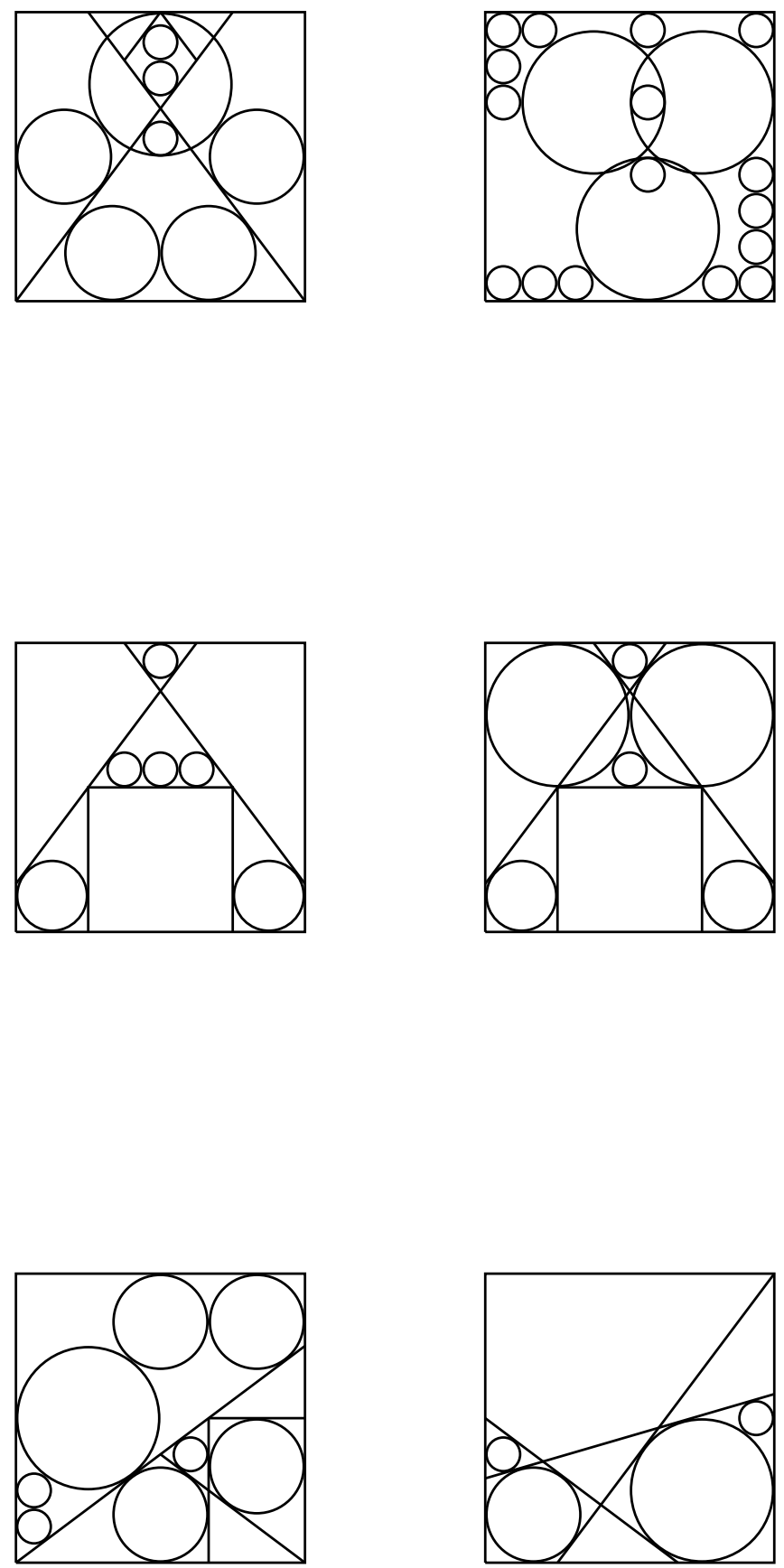

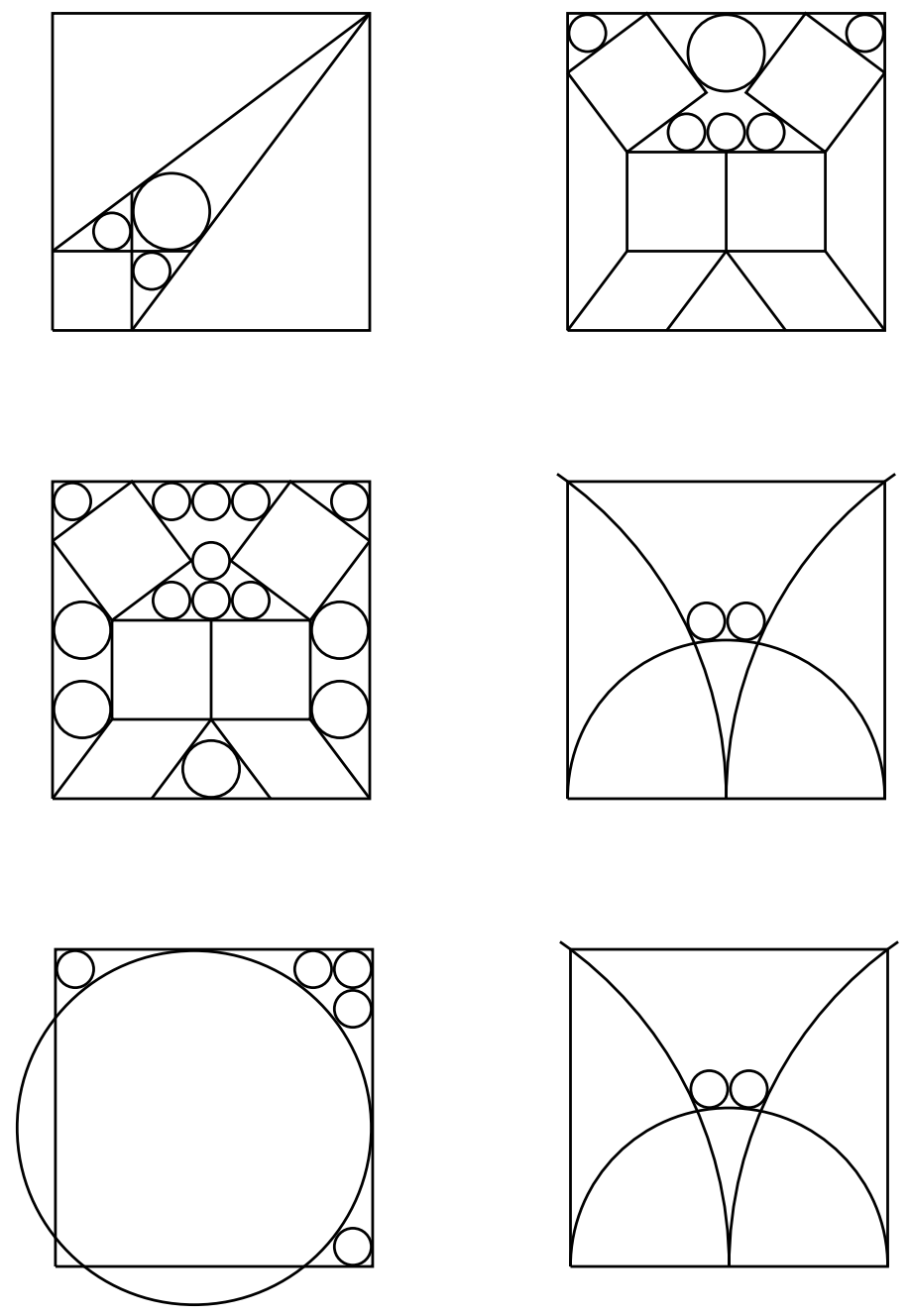


\section{Bibliography}

[1] I. Adler, Make up your own card tricks, Journal of Recreational Math., 6 (1973) 87-91.

[2] I. Affleck and H. L. Abbott, Problem 1884, Crux Math., 19 (1993) 264; solution, 20 (1994) 231-233.

[3] M. Aigner and G. M. Ziegler, Proofs from the BOOK, Springer, 1998.

[4] P. R. Allaire and A. Cupillari, Artemis Martin: An amateur mathematician of the nineteenth century and his contribution to mathematics, College Math. Journal, 31 (2000) 22-34.

[5] G. E. Andrews, A note on partitions and triangles with integer sides, American Math. Monthly, 86 (1979) 477-478.

[6] T. M. Apostol, A property of triangles inscribed in rectangles, Crux Math., 3 (1977) 242-244.

[7] Y. Arzoumanian and W. O. J. Moser, Enumerating 3-, 4-, 6-gons with vertices at lattice points, Crux Math., 19 (1993) 249-254; 279-284.

[8] D. F. Bailey, Counting arrangements of 1's and -1's, Math. Mag., 69 (1996) 128-131.

[9] W. W. R. Ball and H. S. M. Coxter, Mathematical Recreations and Essays, 13th edition, Dover, 1987.

[10] S. Beatty et al., Problem 3177, Amer. Math. Monthly, 33 (1926) 159; solution, 34 (1927) 159-160.

[11] A. Beiler, Recreations in the Theory of Numbers, Dover, 1963. 
[12] A. T. Benjamin and J. J. Quinn, Recounting Fibonacci and Lucas identities, College Math. Journal, 30 (1999) 359-366.

[13] A. T. Benjamin and J. J. Quinn, Proofs that Really Count: The Art of Combinatorial Proof, Math. Assoc. Math., forthcoming.

[14] A. T. Benjamin and J. J. Quinn, The Fibonacci numbers - e

[15] A. Bliss et al., Four constants in four 4s, Math. Mag., 74 (2001) 272.

[16] A. Bourbeau, H. L. Nelson, et al., Problem 142, Crux Math., 2 (1976) 93; solution, 175-178, 3 (1977) 106-108.

[17] A. L. Bouton, Annals of Math., ser. 2, 3 (1902) 33-59.

[18] W. G. Brady and C. W. Twigg, Problem 232, Pi Mu Epsilon Journal, 5.2 (1969) 24; solution 5.3 (1970) 139.

[19] I. Bruce, Another instance of the golden right triangle, Fibonacci Quarterly, 32 (1994) 232-233.

[20] M. Buckheimer and A. Arcavi, Farey series and Pick's area formula, Math. Intelligencer, 17:4 (1993) 64-67.

[21] R. H. Buchholz and R. L. Rathbun, An infinite set of Heron triangles with two rational medians, American Math. Monthly, 104 (1997) 107-115.

[22] P. R. Buckland, The mathematical background of teachers in training, Math. Gazette, 53 (1969) 357-362.

[23] S. Bulman-Fleming and E. T. H. Wang, Problem 1143, Crux Math., 12 (1986) 107; solution, 13 (1987) 237-238.

[24] F. Burk, Natural logarithms via long division, College Math. Journal, 30 (1999) 309-310.

[25] C. K. Caldwell, Truncatable primes, Journal of Recreational Math., 19 (1987) 30-33.

[26] C. K. Caldwell, Near repdigit primes, Journal of Recreational Math., 21 (1988) 299-304; (1989) 101-109.

[27] C. K. Caldwell and H. Dubner, The near-repunit primes $1_{n-k-1} 0_{1} 1_{k}$, Journal of Recreational Math., 27 (1995) 35-41. 
[28] C. K. Caldwell and H. Dubner, Unique-period primes, Journal of Recreational Math., 29 (1998) 43-48.

[29] L. Carroll, Pillow Problems and A Tangled Tale, 1895 and 1885, Dover reprint, 1958.

[30] D. Cass and G. Wildenberg, A novel proof of the infinitude of primes, revisited, Math. Mag., 76 (2003) 203.

[31] P. L. Chessin and W. B. Carver, Problem E 1111, American Math. Monthly, 61 (1954) 258; solution 712.

[32] A. J. Cole and A. J. T. Davie, A game based on the euclidean algorithm and a winning strategy for it, Math. Gazette, 53 (1969) 354-357.

[33] D. B. Coleman, Sketch, a geoboard game, Math. Mag., 51 (1978) 49-54.

[34] J. H. Conway and R. K. Guy, The Book of Numbers, Springer, 1996.

[35] R. J. Covill and T. O'Keeffe, Problem 679, Journal of Recreational Math., 10 (1977-1978) 285; solution, 11 (1978-1979) 312.

[36] H. S. M. Coxeter, The golden section, phyllotaxis, and Wythoff's game, Scripta Mathematica, 19 (1953) 135-143.

[37] K. David, Rencontres reencountered, College Math. Journal, 19 (1988) 139-148.

[38] M. N. Desphande, An unexpected encounter with the Fibonacci numbers, Fibonacci Quarterly, 32 (1994) 108 - 109.

[39] D. W. DeTemple, The triangle of smallest perimeter which circumscribes a semicircle, Fibonacci Quarterly, 30 (1992) 274.

[40] C. Dodge, T. Schoch, P. Y. Woo, and P. Yiu, Those ubiquitous circles, Math. Mag., 72 (1999) 202-213.

[41] H. G. Dworschak and L. Sauvé, Problem 19, Crux Math., 1 (1975) 8; solution, 1 (1975) 32-33.

[42] H. Dubner, Recursive prime generating sequences, Journal of Recreational Math., 29 (1998) 170-175. 
[43] H. E. Dudeney, Amusements in Mathematics, 1919, Dover reprint, 1958.

[44] H. E. Dudeney, 536 Curious Problems and Puzzles, edited by M. Gardner, Barnes and Noble reprint, 1995.

[45] H. E. Dudeney, Canterbury Puzzles, 1919, Dover reprint, 2002.

[46] U. Dudley, Mathematical Cranks, Math. Assoc. America, 1992.

[47] H. G. Dworschak and L. Sauve, Problem 19, Crux Math., 1 (1975) 8 ; solution 32 .

[48] E. J. Eckert, Primitive Pythaogrean triples, College Math. Journal, 23 (1992) 413-417.

[49] E. J. Eckert and H. Haagensen, A Pythagorean tiling of the plane, Math. Mag., 62 (1989) 175.

[50] R. E. Edwards et al., Problem 889, Math. Mag., 47 (1974) 46-47; solution 289-292.

[51] R. B. Ely, III, Magic designs, Journal of Recreational Math., 1 (1968) 5-17.

[52] A. Engel, Problem Solving Strategies, Springer, 1998.

[53] R. Epp and T. Furguson, A note on takeaway games, Fibonacci Quarterly, 18 (1980) 300-303.

[54] P. Erdős and G. Szekeres, Some number theoretic problems on binomial coefficients, Australian Math. Soc. Gazette, 5 (1978) 9799.

[55] S. Forman, A serendipitous application of the Pythagorean triplets, College Math. Journal, 23 (1992) 312-314.

[56] R. L. Francis, Mathematical haystacks: another look at repunit numbers, College Math. Journal, 19 (1988) 240-246.

[57] A. S. Fraenkel and H. Herda, Never rush to be first in playing nimbi, Math. Mag., 53 (1980) 21-26.

[58] H. Fukagawa and D. Pedoe, Japanese Temple Geometry, Charles Babbage Research Centre, Wennipeg, 1989. 
[59] H. Fukagawa and J. F. Rigby, Traditional Japanese Mathematical Problems, 2002, SCT Publishing, Singapore.

[60] H. Fürstenberg, On the infinitude of primes, American Math. Monthly, 62 (1955) 353.

[61] J. Gallego-Diaz and D. L. Silverman, Problem 1453 (corrected), American Math. Monthly, 68 (1961) 177; correction, 572; solution 69 (1962) 166.

[62] J. A. Gallian, The mathematics of identification numbers, College Math. Journal, 22 (1991) 194-202.

[63] M. Gardner, Mathematical card tricks, Scripta Math., 14 (1948) 99-111.

[64] M. Gardner, Hexaflexagons and Other Mathematical Diversions, The First Scientific American Book of Mathematical Puzzles and Diversions, University of Chicago Press, 1988.

[65] M. Gardner, The Second Scientific American Book of Mathematical Puzzles and Diversions, University of Chicago Press, 1987.

[66] M. Gardner, The Colossal Book of Mathematics, Norton, 2001.

[67] R. Gauntt and G. Rabson, The irrationality of $\sqrt{2}$, American Math. Monthly, 63 (1956) 247.

[68] S. Golomb, Permutations by cutting and shuffling, SIAM Review, 3 (1961) 114-118.

[69] S. Golomb, Iterated binomial coefficients, American Math. Monthly, 87 (1980) 719 - 727.

[70] S. Golomb, Problem 477, Pi Mu Epsilon Journal, 7.3 (1980) 187; solution, 8.5 (1981) 348-349.

[71] S. Golomb and M. S. Klamkin, Problem 232, Pi Mu Epsilon Journal, 5.2 (1970) 87; solution, 5.4 (1971) 208.

[72] R. K. Guy, Problem 1153, Crux Math., 12 (1986) 139; solution, 13 (1987) 286-287.

[73] R. K. Guy, Problem 1220, Crux Math., 13 (1987) 54; solution, 14 (1988) 125-126. 
[74] R. K. Guy, The strong law of small numbers, Amer. Math. Monthly, 95 (1988) 697-712.

[75] R. K. Guy and M. E. Marcin, Problem 1915, Crux Math., , 20 (1994) 48; solution, 21 (1995) 30.

[76] R. K. Guy and C. Springer, Problem 1367, Crux Math., 14 (1988) 202; solution, 15 (1989) 278-279.

[77] R. K. Guy, The second strong law of small numbers, Math. Mag., 63 (1990) 3-20.

[78] R. K. Guy, Unsolved Problems in Number Theory, 2nd edition, Springer, 1994.

[79] R. K. Guy and R. E. Woodrow, The Lighter Side of Mathematics, MAA, 1994.

[80] A. Hall, Genealogy of Pythagorean triples, Math. Gazette, 54 (1970) 377-379.

[81] G. H. Hardy, A Mathematician's Apology, Cambridge Univesity Press, 1940.

[82] G. H. Hardy, A Course in Pure Mathematics, 10th edition, 1952, Cambridge; reprint 1963.

[83] A.P. Hatzipolakis and P. Yiu, The Lucas circles of a triangle, Amer. Math. Monthly, 108 (2001) 444 - 446.

[84] O. Higgins, Another long string of primes, Journal of Recreational Math., 14 (1981-82) 185.

[85] A. M. Hinz, Pascal's triangle and the tower of Hanoi, Amer. Math. Monthly, 99 (1992) 538-544.

[86] V. Hoggatt and L. Moser, Problem E 861, American Math. Monthly, 56 (1949) 262; solution 57 (1950) 35.

[87] A. Holshouser and H. Reiter, One pile nim with arbitrary move function, Electronic Journal of Combinatorics, 10 (2003) \#N7.

[88] R. Honsberger, Semi-regular lattice polygons, College Math. Journal, 13 (1982) 36-44. 
[89] R. Honsberger, In Pólya's Footsteps, Math. Assoc. America, 1997.

[90] A. F. Horadam, Fibonacci sequences and a geometric paradox, Math. Mag. 35 (1962) 1-11.

[91] L. S. Johnson, Denumerability of the rational number system, amm 55 (1948) 65-70.

[92] J. H. Jordan, R. Walsh, and R. J. Wisner, Triangles with integer sides, American Math. Monthly, 86 (1979) 686-688.

[93] S. Kahan, s-transposable integers, Math. Mag., 49 (1976) 27-28.

[94] D. Kalman and R. Mena, The Fibonacci numbers - exposed, Math. Mag., 76 (2003) 167-181.

[95] C. Kicey and S. Goel, A series for ln k, American Math. Monthly, 105 (1998).

[96] M. S. Klamkin and P. Watson, Triangulations and Pick's theorem, Math. Mag., 49 (1976) 35-37.

[97] D. A. Klarner, Mathematical Recreations, A Collection in Honor of Martin Gardner, Dover, 1998, originally published as Mathematical Gardner, Wadsworth International, CA, 1981.

[98] M. Kleber, The best card trick, Math. Intelligencer, 24 (2002) 1:911.

[99] D. E. Knuth, Fundamental Algorithms, 2nd edition, AddisonWesley, 1973.

[100] T. Koshy, The digital root of a Fermat number, Journal of Recreational Math., 31 (2002-2003) 112-113.

[101] M. Kraitchik, Mathematical Recreations, 2nd edition, 1942; Dover reprint, 1953.

[102] S. Kumar, On Fibonacci sequences and a geometric paradox, Math. Mag., 37 (1964) 221-223.

[103] V. S. Kumar, On the digital root series, Journal of Recreational Math., 12 (1979-80) 267-270. 
[104] M. E. Larson, The eternal triangle - a history of a counting problem, College Math. Journal, 20 (1989) 370-384.

[105] H. Larson and V. G. Feser, Problem 566, Journal of Recreational Math., 9 (1976) 298; solution, 10 (1977-78) 311-313.

[106] W. G. Leavitt, Repeating decimals, College Math. Journal, 15 (1984) 299-308.

[107] D. N. Lehmer, Asymptotic evaluation of certain totient sums, Amer. J. Math. 22 (1900) 293-335.

[108] R. B. Leigh and R. T. Ng, Minimizing aroma loss, College Math. Journal, 30 (1999) 356-358.

[109] J. Lesko, A series for ln k, College Math. Journal, 32 (2001) 119122.

[110] A. Liu, A direct proof of Pick's theorem, Crux Math., 4 (1978) 242-244.

[111] E. Lucas, Récreations Mathématiques, 4 tomes, Gauthier Villars, Paris, 1891, 1896, 1893, 1894.

[112] A. Machado, Nineteenth problems on elementary geometry, Math. Intelligencer, 17:1 (1995) 17-19.

[113] G. Markowsky, Misconceptions about the golden ratio, College Math. Journal, 23 (1992) 2-19.

[114] L. Marvin and F. H. Kierstead, Problem 315, Journal of Recreational Math., 7 (1974) 62; solution 9 (1976-77) 307.

[115] S. W. McInnis, Magic circles, American Math. Monthly, 60 (1953) 347-351.

[116] R. K. Morley and P. Schub, Problem E24, American Math. Monthly, 40 (1933) 111; solution, 425-426.

[117] S. B. Morris, Faro shuffling and card placement, Journal of Recreational Math., 8 (1975) 1-7.

[118] S. B. Morris, The basic mathematics of the faro shuffle, $P u M u$ Epsilon Journal, 6.2 (1975) 85-92. 
[119] W. Moser, It is elementary (combinatorics), Crux Math., 18 (1992) 257-262; 289-295; 19 (1993) 1-2.

[120] D. Nelson, A regular pentagon construction, Math. Gazette, 61 (1977) 215-216.

[121] I. Niven, Note on a paper by L. S. Johnson, Amer. Math. Monthly, 55 (1948) 358.

[122] L. C. Noll and D. I. Bell, $n$-clusters for $1<n<7$, Mathematics of Computation, 53 (1989) 439-444.

[123] A. Porges, A set of Eight Numbers, American Math. Monthly, 52 (1945) 379 - 384

[124] A. S. Posamentier and C. T. Salkind, Challenging Problems in Geometry, Dover, 1988.

[125] C. S. Queen, Palindromes, Fibonacci Quarterly, 31 (1993) 216226.

[126] S. Rabinowitz, Problem 1299, Journal of Recreational Math., 16 (1983-1984) 139.

[127] Note on magic circles, American Math. Monthly, 60 (1953) 640.

[128] V. Revennaugh and R. W. Buckley, Problem 385, Journal of Recreational Math., 8 (1975-76) 137; solution, 10 (1977-78) 288-289.

[129] P. Ribenboim, The New Book of Prime Number Records, Springer, 1996.

[130] J. Roberts, Elementary Number Theory, A Problem Oriented Approach, MIT, 1975.

[131] R. R. Rowe, Carroll's paper folding pillow problem, Journal of Recreational Math., 4 (1971) 192-198.

[132] D. L. Silverman, R. R. Robinson and R. L. Patton, Problems 141, 142, Journal of Recreational Math., 3 (1970) 237; solution, 4 (1971) 222-223.

[133] F. Rubin and V. G. Feser, Problem 1078, Journal of Recreational Math., 14 (1981) 141-142; solution, 15 (1982-1983) 155-156. 
[134] N. Sato, An ambivalent sum, Crux Math., 23 (1997) 290-293.

[135] D. Schattschneider, Counting it twice, College Math. Journal, 22 (1991) 203-211.

[136] J. Schram, A string of 176 primes, Journal of Recreational Math., 15 (1982-83) 168-169.

[137] J. Schram, A recurrence relation for Fermat numbers, Journal of Recreational Math., 16 (1983-84) 195-197.

[138] A. J. Schwenk, Take-away games, Fibonacci Quarterly, 8 (1970) 225-234.

[139] H. Shapiro, An arithmetic function arising from the $\phi$ function, Amer. Math. Monthly, 50 (1943) 18-30.

[140] D. L. Silverman and D. Singmaster, Problem 1017, Journal of Recreational Math., 13 (1980) 298; solution, 14 (1981-1982) 313.

[141] D. Singmaster, Problem 267, Journal of Recreational Math., 6 (1973) 152-153.

[142] D. Singmaster and B. Barwell, Problem 812, Journal of Recreational Math., 13 (1980-1981) 62-63.

[143] D. Singmaster, Triangles with integer sides and sharing barrels, College Math. Journal, 21 (1990) 278-285.

[144] J. Slocum, The Tangram Book, Sterling, New York, 2003.

[145] C. A. B. Smith, Compound games with counters, Journal of Recreational Math., 1 (1968) 67-77.

[146] M. Somos and R. Haas, A linked pair of sequences implies the primes are infinite, American Math. Monthly, 110 (2003) 539540 .

[147] J. A. Spencer and H. Ahlburg, Problem 428, Crux Math., 5 (1979) 77; solution, 6 (1980) 50.

[148] J. E. Trevor and W. E. Buker, Problem 178, American Math. Monthly, 43 (1937) 104; solution, 499-500.

[149] C. W. Trigg, Eight digits on a cube's vertices, Journal of Recreational Math., 7 (1974) 49-55. 
[150] C. W. Trigg, Tetrahedral models from envelopes, Math. Mag., 51 (1978) 66-67.

[151] C. W. Trigg, Some curiosa involving the first ten perfect numbers, Journal of Recreational Math., 23 (1991) 286.

[152] T. Trotter, Perimeter-magic polygons, Journal of Recreational Math., 7 (1974) 14-20.

[153] M. Wald, Solution to Problem 1664, Journal of Recreational Math., 21 (1989) 236-237.

[154] F. T. Wang and C. C. Hsiung, A theorem on the tangram, American Math. Monthly, 49 (1942) 596-599.

[155] S. R. Wassel, Rediscovering a family of means, Math. Intelligencer, 24.2 (2002) 58-65.

[156] C. Waiveris and Y. Chen, Folding stars, College Math. Journal, 30 (1999) 370-378.

[157] M. Wald, Solution to Problem 1664, Journal of Recreational Math., 21 (1989) 236-237.

[158] J. E. Waltrom and M. Berg, Prime primes, Math. Mag., 42 (1969) 232.

[159] W. C. Waterhouse, Continued fractions and Pythagorean triples, Fibonacci Quarterly, 30 (1992) 144-147.

[160] A. Wayne, A genealogy of $120^{\circ}$ and $60^{\circ}$ natural triangles, Math. Mag., 55 (1982) 157-162.

[161] C. S. Weaver, Geoboard triangles with one interior point, Math. Mag., 50 (1977) 92-94.

[162] D. Wells, Beauty in mathematics, Math. Intelligencer, 10:4 (1988) 31 .

[163] D. T. Whiteside, The Mathematical Papers of Issac Newton, vol. V, Cambridge University Press, 1976.

[164] A. W. Willcox and L. A. Ringenberg, Problem 971, American Math. Monthly, 58 (1951) 417; solution 59 (1952) 105-106. 
[165] P. Woo, Simple constructions of the incircle of an arbelos, Forum Geometricorum, 1 (2001) 133-136.

[166] M. Wunderlich, Another proof of the infinite primes theorem, American Math. Monthly, 72 (1965) 305.

[167] S. Yates, The mystique of repunits, Math. Mag., 51 (1978) 22-28.

[168] S. Yates, Periods of unique primes, Math. Mag., 53 (1980) 314.

[169] S. Yates, Repunits and Repetends, privately published, Delray Beach, FL, 1982.

[170] P. Yiu, Construction of indecomposable Heronian triangles, Rocky Mountain Journal of Mathematics, 28 (1998) 1189 - 1202.

[171] P. Yiu, Mixtilinear incircles, Amer. Math. Monthly, 106 (1999) $952-955$.

[172] P. Yiu, The uses of homogeneous barycentric coordinates in plane euclidean geometry, Int. J. Math. Educ. Sci. Technol, 31 (2000) $569-578$.

[173] P. Yiu, Heronian triangles are lattice triangles, Amer. Math. Monthly, 108 (2001) $261-263$.

[174] P. Yiu, The volume of an isosceles tetrahedron and the Euler line, Mathematics \& Informatics Quarterly, 11 (2001) 15-19.

[175] N. Yoshigahara, Card-return numbers, Journal of Recreational Math., 5 (1972) 36-38. 